\title{
SAMMELN FÜR DIE WISSENSCHAFT?
}

\section{Das Academische Museum Göttingen \\ (1773-1840)}

Christine Nawa

Göttingen 2010 


\section{Inhalt}

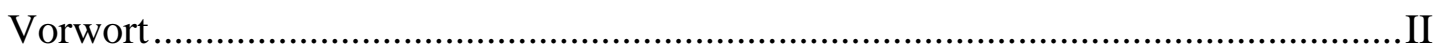

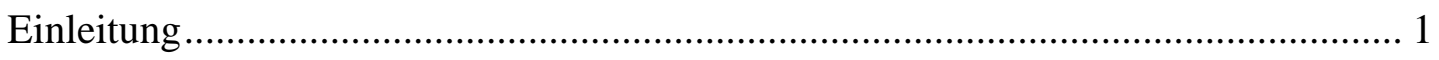

1 Von der Notwendigkeit des Historisierens von Sprache ................................... 12

2 Sammeln im Großen und im Kleinen ............................................................ 29

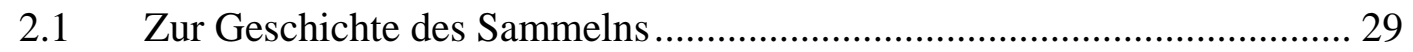

2.2 Das Academische Museum Göttingen - Sammlung und Institution in der Zeit der Gründung und Entwicklung ...................................................... 40

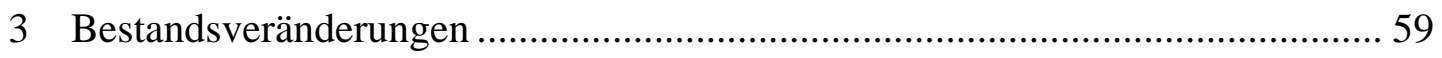

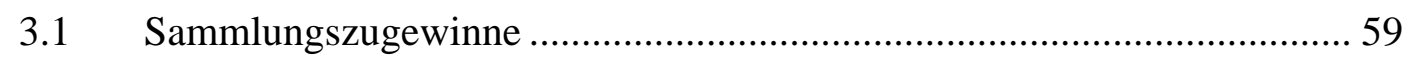

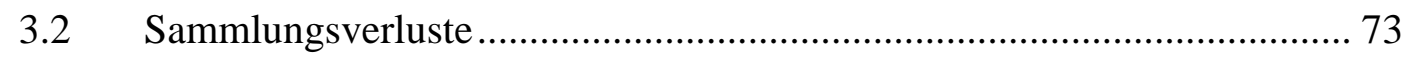

3.3 Eine Ordnung der Dinge und ein neuer Standort..................................... 74

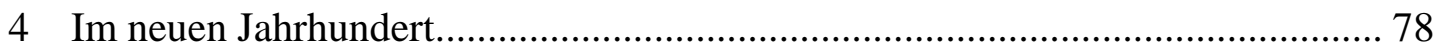

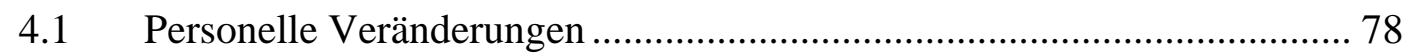

4.2 In der napoleonischen Zeit.............................................................. 79

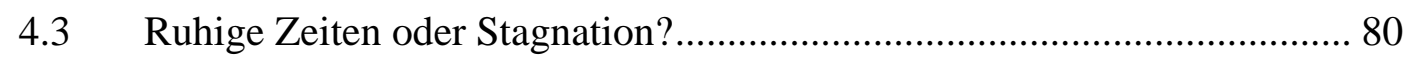

4.4 Das Ende einer Ära und ein Neubeginn - Ausblick auf die Entwicklung nach dem Tod Blumenbachs............................................................... 81

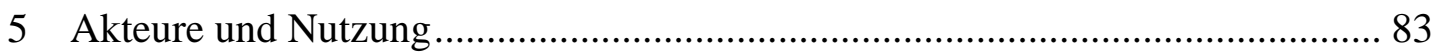

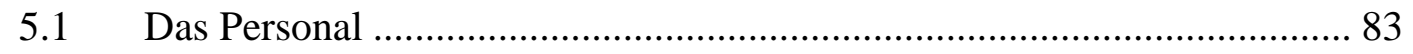

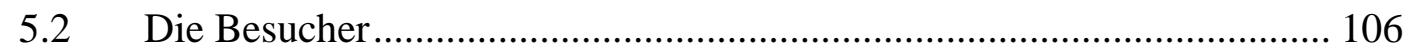

5.3 Zugänglichkeit und Nutzung des Academischen Museums................... 117

Zusammenfassung und Ausblick ..................................................................... 123

Anhang A: Der Catalogus Musei Academici....................................................... 126

Anhang B: Auswertung des Besucherbuchs ........................................................ 135

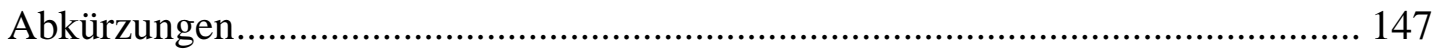

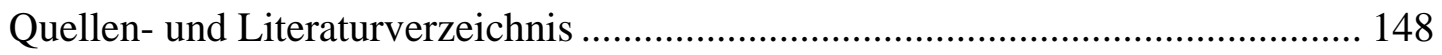

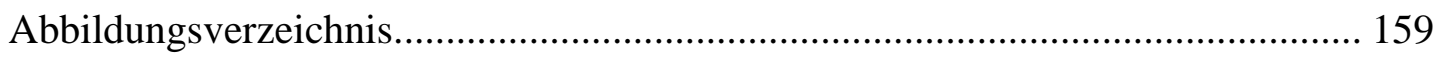




\section{Vorwort}

Bei dem vorliegenden Text handelt es sich um eine überarbeitete Version meiner im Jahr 2005 bei der Philosophischen Universität der Georg-August-Universität Göttingen unter dem gleichen Titel eingereichten Magisterarbeit im Fach Mittlere und Neuere Geschichte.

Nachdem ich in den letzten Jahren vielfach wegen dieser Arbeit angefragt worden bin, habe ich mich nun zu einer Veröffentlichung entschlossen. Da mir die Zeit zu einer umfassenden Überarbeitung fehlt, habe ich entschieden, die Arbeit auf dem damaligen Forschungsstand zu belassen - mit allen Stärken und Schwächen einer Magisterarbeit. Gegenüber der ursprünglich eingereichten Version wurden im Wesentlichen offensichtliche Fehler korrigiert. Größere Änderungen betreffen die datenbankgestützte Auswertung des Besucherbuchs, denn die Datenbank konnte aus technischen Gründen auf diesem Weg nicht mitpubliziert werden. Auch Anhang B ist gegenüber der Ur-Fassung gekürzt.

Einen Überblick über die gesamte Existenzdauer des Academischen Museums (1773-1878), in dem der aktuelle Forschungsstand berücksichtigt ist, gebe ich in dem Artikel „Zum «öffentlichen Gebrauche» bestimmt: Das Academische Museum Göttingen“, der 2011 im Göttinger Jahrbuch erscheinen wird.

Regensburg, im August 2010

Christine Nawa 


\section{Einleitung}

Betrachtet man die aktuellen Ankündigungen für Ausstellungen verschiedener Museen oder auch die für Konferenzen, die sich mit der Museumsgeschichte bzw. der Geschichte des Sammelns befassen, so fällt gegenüber früheren Jahren eine verstärkte Zuwendung zur Selbstreflexivität auf. Dies ist jedoch kein Zufall - stehen doch eine Reihe runder Jubiläen ins Haus, an denen große Häuser ihre Gründung oder ihren Übergang von der Privatsammlung zur öffentlich zugänglichen Sammlung feiern. ${ }^{1}$

Die Institution Museum wird also hinterfragt, es erfolgt eine Besinnung auf die eigene Geschichte. Längst als veraltet abgestempelte Sammlungskonzepte erfahren eine Renaissance - sei es, weil ihnen eine nachträgliche Wertschätzung zuteil wird, sei es, weil die Museen ihrer Geschichte ein Gesicht geben wollen oder weil die zunehmende Interdisziplinarität unter den Fachdisziplinen Sammlungskonzepte aus der Zeit vor der Ausdifferenzierung der einzelnen Lehrfächer als überraschend modern erscheinen lassen.

Grund genug, sich zu vergegenwärtigen, was ein Museum eigentlich ist und wie sich unser heutiges Verständnis davon - möglicherweise - von dem vorangegangener Jahrhunderte unterscheidet. So steht hinter der Leitfrage dieser Arbeit „Sammeln für die Wissenschaft?" letztlich die weitaus größere Frage, was man sich unter einem Universitätsmuseum um 1800 eigentlich vorzustellen hat. Der Titel dieser Arbeit stellt einen Weg der Annäherung an diesen Fragenkomplex dar. Er verweist auf den Zusammenhang von Sammlung und Museum, die Dualität zwischen der Tätigkeit des Sammelns auf der einen und der Sammlung an sich als (repräsentativem) Ergebnis dieser Tätigkeit sowie einer bestimmten, sichtbar gemachten Auffassung von der „Ordnung der Dinge“ ${ }^{2}$ auf der anderen Seite. Dennoch geht es hier nicht um eine Geschichte der Dinge an sich, sondern um die Nutzung des Raumes Museum, der

1 Hier ist zum Beispiel das Herzog Anton Ulrich-Museum in Braunschweig zu nennen, das im Jahr 2004 sein 250-jähriges Bestehen feierte und aus diesem Anlass einen umfangreichen Katalog herausgab. Vgl. Kunstmuseum des LANDES NiEdERSACHSEn Herzog AnTON UlRich-Museum BRAunsCHWEIG (Hg.), 250 Jahre Museum. Von den fürstlichen Sammlungen zum Museum der Aufklärung. Ausstellung in der Burg Dankwarderode, Braunschweig, 29. April bis 22. August 2004, München 2004.

2 Michel Foucault, Die Ordnung der Dinge. Eine Archäologie der Humanwissenschaften, Frankfurt a.M. 2003. 
erst durch das Aufeinandertreffen von Akteuren und Objekten zum Museum wird. Den Untersuchungsgegenstand bildet das 1773 gegründete Academische Museum der Universität Göttingen, das unter eben diesem Namen bis in das letzte Drittel des 19. Jahrhunderts Bestand hatte. Der Untersuchungszeitraum umfasst den mit Ära Blumenbach überschreibbaren Zeitraum von 1773 bis 1840. Blumenbach hatte die Sammlung - wenn auch mit wechselnder Intensität - sein gesamtes akademisches Leben lang begleitet und sie entscheidend geprägt. Er war am Aufschwung der Sammlung ebenso maßgeblich beteiligt wie an ihrer Stagnation. Sein Ableben 1840 bedeutete für die Geschicke des Museums eine starke Zäsur.

Ausgangspunkt dieser Arbeit ist die Frage nach dem Rezipientenkreis des Museums. Wer tritt als Besucher, als Nutzer in Erscheinung, welche Rückschlüsse lassen sich auf den Umgang mit den Objekten ziehen? Was ist eigentlich ein Museumsbesuch? Um sich dieser Frage nähern zu können, ist ein gewisses Vorverständnis für die Entwicklung der Sammlungsgeschichte und des damit verbundenen begrifflichen (historischen) Instrumentariums notwendig, weshalb sich das erste Kapitel in Form eines Glossars einer Betrachtung der wichtigsten für diese Arbeit relevanten Begriffe widmet. Dabei steht nicht der Gedanke einer theoretischen Reflexion im Vordergrund, sondern die Absicht, aus dem zeithistorischen Kontext heraus zu einer Gebrauchsdefinition dieser Begriffe zu gelangen. In diesem Kapitel sollen Bedeutungen zum Teil fremd wirkender Begriffe und die zum Teil fremd wirkenden Bedeutungen einiger bekannter Begriffe herausgestellt werden. Ziel der Auswahl dieser Begriffe ist es, Sensibilität für einige Problematiken der Sammlungsgeschichtsschreibung und der mit ihr verbundenen Terminologie zu wecken. Im darauf folgenden Kapitel, das sich dem Themenkomplex des Sammelns im Großen und im Kleinen widmet, finden diese Begriffe ihre historische Verortung. Hier wird sowohl überblicksartig auf die Geschichte des Sammelns im europäischen Kontext als auch daran anknüpfend - ausführlich auf die Gründungsgeschichte des Academischen Museums Göttingen eingegangen. Dabei stehen der Erwerb der Sammlung und ihre institutionelle Verortung im Mittelpunkt. Die sich unmittelbar nach Gründung des Museums ergebende personelle Situation sollte beinahe vierzig Jahre nahezu unverändert bestehen bleiben. Kapitel 3 setzt Schlaglichter auf die Bestandsveränderungen des Museums und zeigt exemplarisch am Erwerb einer Objektgruppe, der so genann- 
ten Cook-Sammlung, dass sich bei genauerem Hinsehen hinter förmlichen Briefwechseln weit gespannte personelle Beziehungsnetzwerke verbergen können, die für den Erwerb solcher Zugewinne konstitutiv sind. Im Gegensatz zu der ambitionierten Entwicklung des Museums in den Jahren seiner Etablierung zeichnet sich im 19. Jahrhundert eine Phase der Stagnation ab, die zwar von den Wirren der napoleonischen Zeit durchbrochen, aber nicht langfristig aufgehalten wurde. Das vierte Kapitel zeigt auf, dass die Sammlung über lange Jahre mehr verwaltet als entwickelt wurde und dass sich dies erst mit einem längst überfälligen Generationenwechsel am Ende der 1830er Jahre zu ändern begann. In Kapitel 5 werden die Akteure des Museums in den Blick genommen, das Personal desselben mittels Kurzbiographien verortet und der Besucherstrom mittels einer eigens dafür konzipierten Datenbank analysiert. Die Hinzuziehung weiterer Quellen erlaubt es, ausgewählte Besucher plastisch hervortreten zu lassen und der Frage nachzugehen, wie sie auf das Museum aufmerksam geworden sein könnten und welchen Zugangsmodalitäten es unterlag. Zusammenfassend wird kritisch reflektiert, ob das Museum tatsächlich „[n]icht nur der Neugier allein, sondern auch der Natur Lehre nützlich“‘3 gewesen ist - ob also von einem Sammeln für die Wissenschaft gesprochen werden darf.

Insofern versteht sich die vorliegende Arbeit in erster Linie als Beitrag zur Göttinger Universitätsgeschichte, fühlt sich jedoch gleichzeitig der neueren Wissenschaftsgeschichte verpflichtet, die - beginnend mit $\mathrm{Kuhn}^{4}$ und dessen Rezeption - eine Abkehr von der reinen Theorien- und Ideengeschichte vollzogen hat. Seitdem befindet sich die Wissenschaftsgeschichte in einem kontinuierlichen Dynamisierungsprozess, ${ }^{5}$ der - angeregt durch zahlreiche ,Turns ${ }^{3}$ - dazu führt, dass die an neuere wissenschaftshistorische Arbeiten gestellten Ansprüche im Prinzip nur noch durch die Zusammenarbeit eines interdisziplinär zusammengesetzten Autorenkollektivs zu

3 IRMGARD MÜSCH, „Nicht nur der Neugier allein, sondern auch der Natur Lehre nützlich“. Die Naturaliensammlung im Braunschweiger Kabinett, in: Kunstmuseum des Landes Niedersachsen Herzog Anton Ulrich-Museum Braunschweig (Hg.), 250 Jahre Museum. Von den fürstlichen Sammlungen zum Museum der Aufklärung. Ausstellung in der Burg Dankwarderode, Braunschweig, 29. April bis 22. August 2004, München 2004, 61-69.

4 Thomas S. KunN, Die Struktur wissenschaftlicher Revolutionen. Zweite, revidierte und um das Postscriptum von 1969 ergänzte Auflage, Frankfurt a.M. 1976.

5 Vgl. Michael Hagner (Hg.), Ansichten der Wissenschaftsgeschichte, Frankfurt a.M. 2001, $20 \mathrm{f}$.

6 Beginnend mit dem linguistic turn kam es in den 1990er Jahren zu einer regelrecht inflationären Verwendung des Begriffs turn, so dass heute von practical, experimental, discursive, relativistic, body, pictorial, cultural und iconic turn gesprochen wird. 
bewerkstelligen sind. Zu den zahlreichen Aspekten, ${ }^{7}$ die die neuere Wissenschaftsgeschichte in den Blick zu nehmen bemüht ist, gehören neben dem unmittelbaren wissenschaftlichen Betrieb zunehmend lebensweltliche Bezüge wie z.B. das religiöse Leben von Wissenschaftlern ${ }^{8}$ bzw. die Frage, welche Rolle Religiosität in Bezug auf die Ausübung wissenschaftlicher Praktiken spielte. Dazu gehören ebenso das Staunen und Wundern, aber auch Leidenschaft (übergeordnet: Emotionen) einbeziehende Konzepte. ${ }^{9}$

Der Einsicht geschuldet, dass die Naturauffassung der Akteurinnen und Akteure eng mit dem jeweiligen Kulturverständnis zusammenhängt und dass die soziale Rolle der Wissenschaftlerin und des Wissenschaftlers sowie deren bzw. dessen Bemühen um Identitätsfindung die Wahl von Untersuchungsgegenständen und -methoden beeinflusst, wird zunehmend untersucht, welche Bedeutung soziale Praktiken und kulturelle Sinngebungen für die Bildung und Durchsetzung von Theorien haben. ${ }^{10}$ Ebenfalls in diesen Zusammenhang gehört die Frage danach, durch wen Definitionsmacht ausgeübt wird bzw. welche Strategien zum Erwerb von Autorität und Anerkennung für bestimmte Leistungen und/oder Praktiken angewandt werden. Besondere Aufmerksamkeit genießen darüber hinaus Konflikte und Meinungsverschiedenheiten zwischen Forschern sowie das Aufzeigen von Diskontinuitäten. Wechselseitige Einflüsse zwischen Kultur- und Naturwissenschaften, aber auch Brüche im Beziehungsgefüge zwischen Natur- und Humanwissenschaften, zwischen Kultur und Gesellschaft werden zunehmend analysiert. Ebenfalls beispielhaft für die neuere Wissenschaftsgeschichte ist das Konzept einer ,historical geography of science’ ${ }^{11}$

7 Für einen Einblick in aktuelle Forschungstrends stets lohnenswert ist ein Blick auf die Homepage des Max-Planck-Instituts für Wissenschaftsgeschichte in Berlin: http://www.mpiwg-berlin.mpg.de/research.html (Zugriff am 18. Dezember 2004).

8 Vgl. z.B. das am Göttinger Institut für Wissenschaftsgeschichte angesiedelte Projekt Science and Religion. Vgl. http://www.user.gwdg.de/ uhwg/Projekte.html (Zugriff am 18. Dezember 2004).

$9 \quad \mathrm{Zu}$ diesem Themenkomplex und seinem Spannungsverhältnis zu Schlagworten wie Rationalität, Wahrheit und Objektivität hat insbesondere Lorraine Daston wertvolle Beiträge geleistet. Exemplarisch sei hier genannt: LORRAINE DASTON, Die kognitiven Leidenschaften: Staunen und Neugier im Europa der frühen Neuzeit, in: Dies. (Hg.), Wunder, Beweise und Tatsachen. Zur Geschichte der Rationalität, Frankfurt a.M. 2001, 77-98.

10 Hierzu programmatisch der Titel des Sammelbandes: HANS ERICH BöDEKER/Peter HanNS REILL/JÜRGEN SCHLUMBOHM (Hg.), Wissenschaft als kulturelle Praxis. 1750-1900, Göttingen 1999.

11 Dieses Konzept wurde jüngst mit direktem Göttinger Bezug in dem Tagungsband NiCOLAAs RuPKE (Hg.), Göttingen and the Development of the Natural Sciences, Göttingen 2002, stark gemacht. Vgl. darin insbesondere der Aufsatz von CHARLEs WiTHERS, The Geography of Scientific Knowledge, 9-18. 
Zu den in der aktuellen Forschung besonders breiten Raum einnehmenden Aspekten gehört ferner die Frage, an welchen konkreten Orten wissenschaftliche Praxis stattfand. ${ }^{12}$ Die alltäglichen Formen wissenschaftlicher Praxis finden dabei ebenso die Aufmerksamkeit der Forschenden wie die Gestaltung und Zelebrierung von Erkenntnisprozessen. Zunehmendes Gewicht wird der Beziehung zwischen Wissenschaft und (außerwissenschaftlicher) Öffentlichkeit eingeräumt. ${ }^{13}$ Hier ergeben sich direkte Anknüpfungspunkte zur vorliegenden Arbeit.

FORSCHUNGSÜBERBLICK. Das Sammeln als zentrales, elementare ebenso wie ästhetische Bedürfnisse des Menschen ansprechendes Thema hat in den letzten Jahren sowohl in der Wissenschaft als auch in der breiten Öffentlichkeit einen starken Aufschwung erfahren. ${ }^{14}$ Beginnend mit der Wiederentdeckung von Schlossers Werk Die Kunst- und Wunderkammern der Spätrenaissance ${ }^{15}$ in den 1980er Jahren wurde dem Sammlungswesen binnen kürzester Zeit - zunächst in der Kunstgeschichte, ${ }^{16}$ jedoch bemerkenswert schnell auch in anderen Disziplinen, vor allem in der Wissenschaftsgeschichte - ein hohes Maß an Aufmerksamkeit zuteil. Zunächst erhielten die Kunstund Wunderkammern besondere Beachtung - auch und gerade als Geburtsstätten der heutigen Museen. ${ }^{17}$ Diese forderten durch ihre Anordnung, die Fülle ihrer Sammlungsgegenstände und eine stark visuell ausgerichtete Präsentationsform als „Welt in

12 Hier ist zu denken an Bibliotheken, Museen, Seminare, Labore, Kliniken u.a.m. Vgl. zur Verknüpfung von Orten und Praktiken bspw. Bonnie G. SMith, The Practices of Scientific History, in: Dies. (Hg.), The Gender of History: Men, Women, and Historical Practice, Cambridge/Mass. u.a.1998, 103-129, oder auch Helmut Zedelmaier/Martin Mulsow (Hg.), Die Praktiken der Gelehrsamkeit in der Frühen Neuzeit, Tübingen 2001.

13 Vgl. z. B. Constantin GoschleR (Hg.), Wissenschaft und Öffentlichkeit in Berlin 1870-1930, Stuttgart 2000, und MiTCHELl G. AsH, Wissenschaftspopularisierung und bürgerliche Kultur im 19. Jahrhundert, in: Geschichte und Gesellschaft 28 (2002) H. 2, 322-334.

14 Einen guten und vergleichsweise aktuellen Einstieg in die Forschungslandschaft rund um die Geschichte des Sammelns bietet der 2001 erschienene Sammelband von Anke te Heesen und Emma Spary. Während der einleitende Aufsatz der beiden Herausgeberinnen behutsam in die Geschichte des Sammelns einführt und einen ersten Überblick über relevante Entwicklungen und Trends verschafft, führen die nachfolgenden Beiträge die Breite des Spektrums der Sammlungsgeschichte anschaulich vor Augen. Ein theoretischer Abriss Jardines über das wechselvolle Verhältnis von Sammlungen, Wissenschaft und Kultur schließt den Band ab. Vgl. ANKE TE HEESEN/E.C. SpARY (Hg.), Sammeln als Wissen. Das Sammeln und seine wissenschaftsgeschichtliche Bedeutung, Göttingen 2001.

15 Julius VON SCHLOSSER, Die Kunst- und Wunderkammern der Spätrenaissance. Ein Beitrag zur Geschichte des Sammelwesens, Leipzig 1908.

16 Vgl. hierzu Horst BREDEKAMP, Antikensehnsucht und Maschinenglauben. Die Geschichte der Kunstkammer und die Zukunft der Kunstgeschichte, Berlin 1993.

17 Oliver Impey/Arthur MacGregor (Hg.), The Origins of Museums. The Cabinet of Curiosities in sixteenth- and seventeenth-century Europe, Oxford 1986. 
der Stube“ ${ }^{18}$ geradezu zu einer vom Objekt her verstandenen Geschichtsschreibung heraus, in der materiale und visuelle Kultur symbiotisch nebeneinander stehen. ${ }^{19}$ Als Sammlungsorte kamen hier vor allem Fürstenhöfe in den Blick. ${ }^{20}$

Als zweiter Interessenschwerpunkt kristallisieren sich die Entstehungsgeschichten international bekannter Museen auf der einen Seite und die große Welle der Museumsgründungen im ausgehenden 19. Jahrhundert auf der anderen Seite heraus. ${ }^{21}$ Hier bildet die Entwicklung naturhistorischer Museen einen großen Schwerpunkt. Zunehmende Beachtung erfährt auch der Konnex zwischen Ausstellung und Ausstellungsraum. $^{22}$ Dominierten zunächst Untersuchungen aus dem englischen Sprachraum, so erschienen seit Mitte der neunziger Jahre auch in Deutschland verstärkt Publikationen zur Sammlungsgeschichtsforschung. Damit verschob sich der Interessenschwerpunkt erneut: Vermehrt gerieten die Geschichte der Naturalienkabinette bzw. allgemein die Sammlungskultur im 18. Jahrhundert und damit auch bürgerliche Privatsammlungen in den Blick. ${ }^{23}$ Somit wurde die Lücke zwischen Renaissance und Historismus geschlossen. An dieser Stelle verschob sich gleichzeitig der Fokus weg von der materialen Kultur, von einer vom Objekt her verstandenen Geschichtsauffassung hin zu den Akteuren und den Praktiken des Sammelns. Dennoch können als theoretische Eckpfeiler der Sammlungsforschung weiterhin die wegweisende Arbeit Pomians ${ }^{24}$ und die inzwischen klassische Abhandlung Foucaults ${ }^{25}$ gelten. ${ }^{26}$

18 Andreas Grote (Hg.), Macrocosmos in Microcosmo. Die Welt in der Stube. Zur Geschichte des Sammelns 1450 bis 1800, Opladen 1994.

19 Vgl. hierzu insbesondere KRZYSZTOF POMiAn, Collectionneurs, amateurs et curieux. Paris, Venise, XVIe-XVIIIe siècle, Paris 1987.

20 Vgl. bspw. EliSABETH SCHEICHER, Die Kunst- und Wunderkammern der Habsburger. Herausgegeben von Christian Brandstätter, Wien - München - Zürich 1979.

21 Vgl. z. B. Tony Bennett, The Birth of the Museum. History, Theory, Politics, London u.a.1997.

22 Beispielhaft: CARla Yanni, Nature's Museums. Victorian Science and the Architecture of Display, Baltimore 1999.

23 Vgl. hierzu Claudia Valter, Studien zu bürgerlichen Kunst- und Naturaliensammlungen des 17. und 18. Jahrhunderts in Deutschland, Aachen 1995; DIES., Kunst- und Naturalienkabinette in der ersten Hälfte des 18. Jahrhunderts, in: Kunstmuseum des Landes Niedersachsen Herzog Anton Ulrich-Museum Braunschweig (Hg.), 250 Jahre Museum. Von den fürstlichen Sammlungen zum Museum der Aufklärung. Ausstellung in der Burg Dankwarderode, Braunschweig, 29. April bis 22. August 2004, München 2004, 21-30; CHRISTOPH BECKER, Vom Raritäten-Kabinett zur Sammlung als Institution. Sammeln und Ordnen im Zeitalter der Aufklärung, Egelsbach u.a.1996; STEFAN SIEMER, Geselligkeit und Methode. Naturgeschichtliches Sammeln im 18. Jahrhundert, im Erscheinen. Stefan Siemer möchte ich an dieser Stelle einen herzlichen Dank für das Bereitstellen des Manuskriptes seiner Arbeit aussprechen.

24 POMian, Collectionneurs.

25 Foucault, Die Ordnung der Dinge. 
Paradigmatisch für die Sammlungsgeschichtsschreibung ist, dass sie nach wie vor wenige umfassende Geschichten des Sammelns, ${ }^{27}$ sondern vielmehr Geschichten einzelner Sammlungen oder auch Museen schreibt. Besondere Bedeutung in Hinblick auf die neuere Sammlungsgeschichtsschreibung kommt deshalb dem Ausstellungsbandband Theater der Natur und Kunst ${ }^{28}$ zu. Er versucht als erster Katalog seiner Art, die Sammlungen einer Universitätsstadt, nämlich Berlin, in ihrer Gesamtheit in den Blick zu nehmen. Diesem Beispiel folgte, allerdings etwas anders aufgebaut, ein Jahr später auch der Band zu den Sammlungen der Universität Göttingen, in dem auch die aus dem Academischen Museum hervorgegangenen Sammlungen ihren Platz finden. ${ }^{29}$

Wiewohl in diesem Band im Zusammenhang der Entstehungsgeschichten zahlreicher Göttinger Sammlungen auf das Academische Museum rekurriert wird, steht eine Einzeldarstellung hierzu bislang noch aus. Generell hat diese Institution von Seiten der Geschichtswissenschaft bislang keine Aufmerksamkeit erfahren. Vielmehr wurde dem Museum vor allem dann ein gesteigertes Interesse zuteil, wenn es darum ging, von der Geschichte der aus dem Academischen Museum hervorgegangenen Einzelsammlungen zu berichten, allen voran von der Erwerbungsgeschichte der CookForster-Sammlung. Um die Aufarbeitung der Geschichte des Academischen Museums innerhalb solcher Darstellungen haben sich vor allem die Direktoren bzw. Kustoden der Völkerkundlichen Sammlung der Universität Göttingen verdient gemacht. Insbesondere ist hier die Arbeit von Hans Plischke, dem ersten Institutsdirektor, aus dem Jahr 1931 zu erwähnen, in der er auf der Grundlage eines ausgiebigen Quellenstudiums in erster Linie die frühe Geschichte des Museums darstellt und bereits zu

26 Wider Erwarten ist die Sammlungsgeschichte in der allgemeinhistorischen Zeitschriftenliteratur unterrepräsentiert. Hingegen existieren einige fachspezifische Zeitschriften. So informiert bspw. das seit 1989 erscheinende Journal of the History of Collections (Oxford University Press, herausgeben von OLIVER IMPEY/ARTHUR MACGREGOR) regelmäßig über verschiedene Sammlungen. Zudem stellt das Internet eine zunehmend wichtiger werdende Plattform für museumsspezifische Foren dar. Vgl. z. B. H-Net Museum (http://www.h-net.org/ museum/ (Zugriff am 1. Januar 2005)) oder Museologie Online (http://www.vl-museen.de/m-online/ (Zugriff am 1. Januar 2005)).

27 Diesem Trend entgegenwirkend: SuSAN M. PEARCE, Museums and their development. The European Tradition 1700 - 1900, London 1999.

28 Horst BredeKamp/JoAchim BrÜning/Cornelia Weber (Hg.), Theater der Natur und Kunst. Wunderkammern des Wissens. Essays. Begleitband zur Ausstellung Theatrum naturae et artis Theater der Natur und Kunst. Wunderkammern des Wissens, Martin-Gropius-Bau Berlin, 10. Dezember 2000 bis 4. März 2001, Berlin 2000.

29 Dietrich Hoffmann/Kathrin MAACK-Rheinländer (Hg.), „Ganz für das Studium angelegt“. Die Museen, Sammlungen und Gärten der Universität Göttingen, Göttingen 2001. 
diesem verhältnismäßig frühen Zeitpunkt auf den wissenschaftsgeschichtlichen Wert der Sammlung hinweist. ${ }^{30}$ Ebenfalls unverzichtbar ist der Band Göttinger Zoologen von Ernst Ehlers, ${ }^{31}$ der bereits 1901 erschien. Wertvolle Ergänzungen in Detailfragen bieten die Arbeiten Manfred Urbans ${ }^{32}$ und Gundolf Krügers ${ }^{33}$, zuletzt 2000, beide ehemalig bzw. aktuell Kustoden der Völkerkundlichen Sammlung. Eine Annäherung an die (Bild-)Bestände des Museums wurde jüngst von Kerstin Dudley ${ }^{34}$ unternommen. Insgesamt ist die Forschungsliteratur zu diesem Thema jedoch sehr überschaubar. Die vorliegende Arbeit versteht sich als experimentelle, quellenbasierte Grundlagenarbeit, zu deren zentralen Anliegen die Erschließung von Beständen für weitere forschungsrelevante Tätigkeit gehört.

QUELLENBERICHT. Die vorliegende Arbeit basiert in wesentlichen Teilen auf ungedruckten Quellen. Die wichtigste unter ihnen wird im Archiv der Völkerkundlichen Sammlung der Universität Göttingen aufbewahrt. ${ }^{35}$ Hierbei handelt es sich um das Besucherbuch ${ }^{36}$ des Academischen Museums, das für den Zeitraum 1808 bis 1837 die „Nahmen der Fremden respective, welche das Museum besuchen“ und darüber hinaus oft auch Herkunftsorte und Berufe dieser Personen enthält. Dieses Buch

30 HANS PlischKe, Die Ethnographische Sammlung der Universität Göttingen. Ihre Geschichte und ihre Bedeutung, Göttingen 1931, 5.

31 E[RNST HEINRICH] EHLERS, Göttinger Zoologen, Berlin 1901.

32 Vgl. MANFRED URBAN, Die Erwerbungsgeschichte der Göttinger Sammlung, in: Brigitta HauserSchäublin/Gundolf Krüger (Hg.), James Cook: Gifts and Treasures from the South Seas. Gaben und Schätze aus der Südsee. The Cook/Forster Collection, Göttingen, Göttingen 1998, 56-85; DERS., Die Völkerkundliche Sammlung, in: Hoffmann/Maack-Rheinländer (Hg.), „Ganz für das Studium angelegt“, 91-98. DERS., Frühe Auswertung des „Ethnographischen Apparates“ im Hochschulunterricht zu Beginn des 19. Jahrhunderts, in: 250 Jahre Georg-August-Universität Göttingen. Ausstellung im Auditorium 19. Mai - 12. Juli 1987, Göttingen 1987, 158-160.

33 GundOLF KRÜGER, „Alles Geflechte besonders schön“. Begegnung mit Ethnographica des Academischen Museums, in: Elmar Mittler (Hg.), „Der gute Kopf leuchtet überall hervor“. Goethe, Göttingen und die Wissenschaft, Göttingen 1999, 66-72; GUNDOLF KRÜGER, Die Völkerkundliche Sammlung Göttingen. Eine Forschungsstätte an der Schnittstelle zwischen Museum und Universität. Vortrag, gehalten auf der Jahrestagung des Museumsverbandes für Niedersachsen und Bremen e.V. am 16. März 2000 in Göttingen, in: Mitteilungsblatt. Museumsverband Niedersachsen Bremen 59 (2000), 91-98.

34 Vgl. KeRSTIN DudLEY, Historische Abbildungen der Völkerkundlichen Sammlung der Universität Göttingen aus der Zeit des Academischen Museums (1773-1840), (unveröffentlichte Magisterarbeit) 2 Bde, Göttingen 2001. Die Untersuchung erfolgte datenbankgestützt.

35 Das Archiv der Völkerkundlichen Sammlung ist nicht öffentlich zugänglich, jedoch wurden mir dessen Bestände für meine Recherchen in großzügiger Weise zugänglich gemacht, wofür ich mich an dieser Stelle herzlich bedanken möchte.

36 Die für das Buch angewandte Terminologie ist nicht einheitlich, da es keinen Titel besitzt. Die dafür in der Forschung benutzten Begriffe Gästebuch, Fremdenbuch und Besucherbuch werden in dieser Untersuchung synonym verwendet. 
wurde bislang nicht systematisch ausgewertet; im fünften Kapitel dieser Arbeit wird damit begonnen. Ein Erörterung dazu, dass das Besucherbuch nicht nur die wichtigste, sondern zugleich auch die problematischste Quelle darstellt, ist, um den Komplex in einer funktionellen Einheit zu belassen, in Kapitel 5 enthalten.

Zu weiteren Akten aus dem Archiv der Völkerkundlichen Sammlung, die für diese Arbeit herangezogen wurden, gehören der Catalogus Musei Academici, ${ }^{37}$ ein handschriftlicher Katalog der Bestände des Museums aus dem Jahr 1778 (in Erst- und Zweitausfertigung), sowie zahlreiche Verwaltungsakten in Briefform. Diese befassen sich für den Untersuchungszeitraum vor allem mit der Akquise von Sammlungsmaterial. Hier ist beispielsweise der Erwerb der Cook-Forster-Sammlung in hervorragender Weise überliefert.

Ferner wurden in großem Umfang Akten des Göttinger Universitätsarchivs herangezogen. Hierbei handelt es sich um Kuratorialakten, also ausschließlich um Akten, die im Rahmen amtlicher Tätigkeit entstanden, und sich vor allem aus Schreiben und Rechnungen zusammensetzen. Ausgewertet wurden gut vierzig Akten aus dem Zeitraum 1756 bis $1840 .^{38}$ Sie geben vor allem über die Gründung des Academischen Museums, personelle Veränderungen und Sammlungszugewinne Auskunft.

Die umfangreichen, vor allem Selbstzeugnisse enthaltenden Bestände der Handschriftenabteilung der Niedersächsischen Staats- und Universitätsbibliothek Göttingen konnten in dieser Arbeit nicht berücksichtigt werden. Für weiterführende Forschungen z.B. zur Person Blumenbachs müssten diese Quellen als sehr relevant eingestuft und daher berücksichtigt werden.

Je nach den spezifischen Eigenschaften einzelner Quellengruppen ist eine differenzierte quellenkritische Herangehensweise erforderlich. So erlauben die erhaltenen Museumskataloge Aussagen zum Bestand des Academischen Museums kurz nach seiner Gründung sowie zu der Ordnung, die die Objekte in den Katalogen erhalten haben. Sie schweigen jedoch zu der Frage, wie die Objekte ihren Weg ins Museum

37 Hierbei handelt es sich um den von Blumenbach verfertigten Katalog sowie dessen in Reinschrift von anderer Hand angefertigte Abschrift. Für diese Arbeit wurde nur die von Blumenbach selbst niedergeschriebene Version herangezogen. Andere Bearbeiter verweisen jedoch darauf, dass es bei der Abschrift wohl zu Fehlern gekommen sei. Vgl. MANFrED URBAN, Die Völkerkundliche Sammlung, in: Hoffmann/Maack-Rheinländer (Hg.), „Ganz für das Studium angelegt“, 91-98, 98 EN 4.

38 Der Umfang der einzelnen Akten variiert stark, lässt sich aber insgesamt auf etwa 2900 handbeschriebene Seiten bemessen. Dies machte eine Selektion erforderlich. 
gefunden haben, welcher materielle Wert den einzelnen Objekten zukommt und vor allem auch darüber, ob die Ordnung der Kataloge die Ordnung der Dinge im Museum selbst widerspiegelt. Die Verwaltungsquellen geben elementare Einblicke in die Organisationsstruktur und die funktionellen Abläufe im und um das Museum. Doch weist der erhaltene Quellenkorpus eine gewisse Disparität auf: Zum einen sind zur Frühgeschichte des Museums ungleich mehr Quellen überliefert als für spätere Zeitabschnitte. Zum anderen liegen für verschiedene Zeiträume inhaltlich jeweils stark von einander verschiedene Quellen vor. Der Katalog der Sammlung stammt, wie bereits erwähnt, aus dem Jahr 1778. Ein annähernd vollständig wirkendes Verzeichnis der Sammlungseingänge scheint nur für den Zeitraum 1778 bis 1781 überliefert zu sein. Rechnungen sind aus dem Zeitraum 1814 bis 1840 erhalten, das Besucherbuch wiederum deckt den Zeitraum von 1808 bis 1837 ab. Insbesondere die Schreiben stellen - je nach Anlass - der hannoverschen und auch westphälischen Regierung gegenüber die Situation des Museums unterschiedlich dar - je nachdem, ob man Fördermittel erhalten oder seine eigenen Tätigkeiten lobend herausstellen wollte. Normative Quellen hingegen wie z.B. Dienstanweisungen erlauben zwar Einblicke in Tätigkeitsfelder, gestatten jedoch keine Aussage darüber, inwieweit eine Normumsetzung tatsächlich erfolgte. Rechnungen geben detaillierte Einblicke in die materiellen Bedürfnisse des Museums und seine Ankaufstätigkeit. Sie zeigen Akteure auf, die an anderen Stellen nicht in Erscheinung treten, ${ }^{39}$ geben allerdings keinerlei Informationen zu Beweggründen oder Befindlichkeiten preis.

Die verwendeten gedruckten Quellen gliedern sich in Lexikonartikel, museologische Schriften, die vor allem für die ersten beiden Kapitel von Belang sind, zeitgenössische Zeitschriftenaufsätze ${ }^{40}$ und ,Reiseführer', die Informationen über die Bestände des Museums und die Arbeit mit ihnen liefern, sowie vor allem universitätsgeschichtliche Schriften, die über die Zugänglichkeit des Museums und die Biographien der zentralen Akteure informieren. Vereinzelt wurden auch Selbstzeugnisse herangezogen, um Museumsaufenthalte verifizieren zu können.

Lexika geben Einblicke in zeitgenössische Argumentationsmuster und erlauben begriffliche Historisierungen. Unklar bleibt jedoch, inwieweit die in ihnen getroffenen

39 Hier sind z. B. Händler, Handwerker, Transporteure und sonstige Zulieferer zu nennen.

40 Hierzu zählen insbes. die Artikel in den Göttingischen Anzeigen von Gelehrten Sachen (GGA). 
Aussagen allgemeine Akzeptanz genossen, über welchen Bekanntheits- und Wirkungsgrad ihre definitorischen Bemühungen verfügten und ob hier nicht künstliche Stereotypen geschaffen wurden. Zudem ist zu berücksichtigen, dass einige zeitgenössische Lexika, insbesondere die Encyclopädie, ${ }^{41}$ nicht einfach nur als Nachschlagewerke, sondern auch als Streitschriften der Aufklärung betrachtet werden müssen.

Zeitschriftenartikel und Reiseführer im weitesten Sinne liefern schließlich vor allem Informationen über (exponierte) Objekte des Museums. Durch sie kann man schlussfolgern, welche Informationen einer lesenden Öffentlichkeit im ausgehenden 18. Jahrhundert zuteil werden konnten; wie groß diese war, bleibt allerdings offen. Zudem handelt es sich bei diesen Quellen häufig um Kompilationen, in denen sich neueste Entwicklungen nicht zwangsläufig widerspiegeln müssen.

Die Arbeit gliedert sich in einen Textteil, der sich mit zeitgenössischen sammlungsgeschichtlich relevanten Begrifflichkeiten ebenso auseinandersetzt wie mit der Geschichte des Sammelns im Allgemeinen und der Geschichte des Academischen Museums Göttingen im Speziellen. In einem zweiten Teil erfolgte die Überführung einer umfangreichen handschriftlichen Quelle in eine Transkription, aus der eine vereinheitlichte, zu statistischen Zwecken nutzbare Datenbank ${ }^{42}$ entwickelt wurde.

41 Denis Diderot/JeAn le Rond D'Alembert, Encyclopédie ou Dictionnaire Raisonné des Sciences, des Arts et des Métiers, par une Société de Gens de Lettres. 17 Text- und 11 Tafelbände, Paris 1751-1772.

42 Hier gilt mein besonderer Dank Arnd Beyer für großzügige Unterstützung, hilfreiche Erklärungen und vor allem für seine Engelsgeduld. 


\section{Von der Notwendigkeit des Historisierens von Sprache}

Zu den wohl am häufigsten zitierten Texten die Geschichte des Academischen Museums betreffend gehört die Anfrage Blumenbachs an die Hannoversche Landesregierung, in der er die Anschaffung von Sammlungsobjekten von den Weltumsegelungen James Cooks für das Museum vorschlägt. Demütig erkundigt er sich, ob nicht „etwas von dem Ueberfluße ausländischer Natürlicher Merkwürdigkeiten, welche besonders durch die, auß Ihro Majestät Befehl neuerlich vollzognen Reisen um die Welt in größter Menge gesammelt worden, für das academische Museum zu erhalten stünde?“43 Doch was verbirgt sich hinter diesen Merkwürdigkeiten? Bevor es um die materialen Eigenschaften von Sammlungsobjekten geht, soll die sich wandelnde Semantik epochenübergreifend verwendeter Begriffe in den Blick genommen werden. Zahlreiche Begriffe, die sich auch noch in unserer Alltagssprache finden, waren bei den Zeitgenossen Blumenbachs anders besetzt und bedürfen daher der Erläuterung. Hier ist eine Kontextualisierung, eine Historisierung notwendig. Einige Begriffe, die für diese Arbeit von besonderer Relevanz sind, werden im Folgenden erläutert - vor allem unter Hinzuziehung des Zedler'schen Universal-Lexikons ${ }^{44}$ und der Encyclopédie d'Alemberts und Diderots ${ }^{45}$. Diese beiden Lexika können wohl als die renommiertesten fertig gestellten Lexika des 18. Jahrhunderts gelten und einen verhältnismäßig großen Bekanntheitsgrad für sich beanspruchen. Die Encyclopédie wird vor allem dann herangezogen, wenn die Angaben im Zedler wenig aussagekräftig erscheinen oder wenn die Beiträge der Encyclopédie besonders sprechende Bilder vermitteln. Wo Ergänzungen notwendig erscheinen, wird teilweise auf das Wörterbuch Adelungs ${ }^{46}$ zurückgegriffen. Darüber hinaus werden einige für die Fragestellung relevante Begriffe wie z.B. Wissenschaft näher eingegrenzt. Zuletzt finden einige Begriffe aus dem heutigen Sprachgebrauch Eingang in dieses Glossar, die man-

43 AVkG, Akten 1 - Altakten (lose), Erwerb Cook-Sammlung, Schreiben vom 27. August 1781.

44 JohANN HEINRICH ZEDLER, Großes vollständiges Universal-Lexikon aller Wissenschaften und Künste, [...] Bde 1-64, Suppl.Bde 1-4, Nachdruck der Ausgabe Halle - Leipzig 1732-50 und 17511754, Graz 1961-1964.

45 DiDEROT/D'ALEMBERT, Encyclopédie.

46 JOHANn CHRISTOPH ADELUNG, Grammatisch-kritisches Wörterbuch der Hochdeutschen Mundart, mit beständiger Vergleichung der übrigen Mundarten, besonders aber der Oberdeutschen, Wien 1811. 
gels einheitlicher Terminologie häufig missverstanden ${ }^{47}$ werden und deren Verwendung nachstehend kurz erläutert wird.

\section{Kabinett}

Im heutigen Sprachgebrauch wird das Wort Kabinett nur noch vergleichsweise selten verwendet und ist in der Regel politisch konnotiert oder verweist auf kleine Ausstellungsräume in Museen wie z.B. Kupferstich-Kabinette. In der Frühen Neuzeit dagegen findet der Begriff eine deutlich breitere Anwendung. So bezeichnet er in dieser Zeit Schatzkammern und Rückzugsräume, Ankleidezimmer oder Studierstuben und noch einiges andere mehr. ${ }^{48}$ Vor allem begegnet er aber in Determinativkomposita wie Kunst- und Naturalienkabinett, Kuriositätenkabinett, Raritätenkabinett und dergleichen. Dabei wird der Begriff Kabinett in den Quellen in der Regel synonym zum Begriff Kammer verwendet. Für diese Arbeit ist vor allem der Begriff des Naturalienkabinettes relevant. Auf ihn und sein Verhältnis zum Begriff Kabinett wird weiter unten ausführlicher eingegangen.

\section{Kuriosität}

In Anbetracht der Tatsache, dass Kuriositätenkabinette häufig als Vorläufer heutiger Museen bezeichnet werden, scheint es sinnvoll, den Begriff Kuriosität näher zu umreißen. Interessanterweise findet sich in dem entsprechenden Artikel bei Zedler kein Verweis auf Kuriositäten mit Objektcharakter oder gar Kuriositätenkammern. Im Adelung von 1811 findet sich noch nicht einmal ein Eintrag zu kurios in irgendeiner Form. Dennoch vermittelt der Eintrag zu Neugierigkeit im Zedler einen gewissen Eindruck davon, was man im Zeitalter der Aufklärung unter Curiosität verstand. Dort wird der Begriff der Kuriosität dem der Neugierigkeit gleichgesetzt: „Neugierigkeit, Curiosität, ist eine Art der Wollust, da man nach neuen und ungewöhnlichen Sachen

47 Gerade bei Diskussionen zwischen Angehörigen unterschiedlicher wissenschaftlicher Disziplinen kommt es mangels einheitlicher Terminologien immer wieder zu Missverständnissen. Deswegen postulierte Ilse Jahn jüngst die Notwendigkeit eines einheitlichen begrifflichen Instrumentariums in der Museumsforschung und trat für eine dezidierte Begriffsklärung ein. Diskussion im Rahmen des Symposiums „Zur Geschichte der Museen im 19. Jahrhundert. 1789-1918“, Institut für Museumskunde, Staatliche Museen zu Berlin, Berlin 2./3. Dezember 2004.

48 Im Französischen wohnt diesem Begriff eine besonders große Bedeutungsvielfalt inne. Hier kann er, je nach Nutzung auch als kleines Gesellschaftszimmer oder auch als Raum für kleine Konzerte, im Deutschen würde man wohl Kammerkonzerte sagen, dienen. In jedem Fall stellt ein Kabinett einen kleinen Raum, ein Nebenzimmer dar. 
begierig ist, um sich dadurch zu belustigen, und die Zeit hinzubringen.“49 Es wird kein Zweifel daran gelassen, dass „,[a]uf solche Weise [...] die Curiosiät mit unter die Schwachheiten des menschlichen Willens“"50 zu zählen sei. In moralisierendem Tonfall werden die negativen Aspekte der Neugierigkeit aufgezählt. Doch es wird auch auf eine Ausnahme hingewiesen: Allein die „gelehrte Curiosität“, also das Ergötzen an scharfsinnigen Erfindungen sei - sofern in „gehörigen Schrancken“ - „nicht zu verwerfen“ und „vornehmlich iedem Gelehrten anzupreisen“, denn sie „erwecket Nachdencken“ und „Nachdencken bringet scharfsinnige Wahrheiten hervor“. ${ }^{51}$ Der Artikel endet bezeichnenderweise mit dem Verweis auf die allegorische Darstellung der Neugierigkeit „als ein Frauenzimmer“. 52

Während die heute auch denkbare Konnotation von kurios mit sonderbar durchaus zulässig erscheint, ist die Gleichsetzung mit dem Begriff merkwürdig in diesem Zusammenhang falsch. Der häufig in Quellentexten erscheinende Begriff merkwürdig ist in diesem Kontext im Wortsinne, also als des Merkens würdig, als besonders zu verstehen. ${ }^{53}$ Dieser Gegensatz kommt schön zur Geltung bei Blumenbach, der, sich an eigene Dozenten zu Studienzeiten erinnernd, vom „sonderbaren aber merkwürdigen Büttner“ ${ }^{\text {54 }}$ spricht.

\section{Museum}

Es macht wenig Sinn, bereits hier festzulegen, was es genau bedeutet, wenn sich eine universitäre Sammlung um 1780 den Namen Museum gibt, welche Ansprüche dahinter stehen und wie diese letztlich umgesetzt werden, denn das sind schließlich einige der zentralen, dieser Untersuchung zu Grunde liegenden Fragen. Folglich würde eine so geartete Definition an dieser Stelle unweigerlich einen Zirkelschluss provozieren. Jedoch scheint es sinnvoll zu fragen, wie die eingangs erwähnten zeitgenössischen

49 ZEDLER, Bd 24, 1740, Sp. 172.

ZEDLER, Bd 24, 1740, Sp. 172.

ZEDLER, Bd 24, 1740, Sp. $173 f$.

ZEDLER, Bd 24, 1740, Sp. 174.

53 Der ADELUNG verzeichnet ebenfalls die Erläuterung „würdig, oder werth, gemerket, d.i. im Gedächtnisse behalten zu werden; denkwürdig.“ Vgl. ADELUNG, Grammatisch-kritisches Wörterbuch Bd 3, Sp. 183. Das ZEDLERsche Universal-Lexikon verzeichnet zu diesem Wort keinen Eintrag.

54 KARL Friedrich HeInRICH MARX, Zum Andenken an Johann Friedrich Blumenbach. Eine Gedächtniss-Rede gehalten in der Sitzung der Königlichen Societät der Wissenschaften den 8. Februar 1840, Göttingen 1840, 5. 
Lexika den Museumsbegriff definieren. ${ }^{55}$ Dabei sollen keine Stereotypen festgeschrieben, sondern eine Orientierungsmöglichkeit ${ }^{56}$ für die nachfolgende Untersuchung geschaffen werden. Dass die historischen Akteure dieser Untersuchung die entsprechenden Lexikonartikel kannten ist nicht gesichert, jedoch nicht unwahrscheinlich. Setzt man eine solche Kenntnis voraus, könnte sie Einfluss auf die Namensgebung gehabt haben. ${ }^{57}$

Die Grundaussage zum Museumsbegriff stimmt bei Zedler und der Encyclopédie überein. Beide rekurrieren auf das Museum Alexandrinum, den aus dem dritten vorchristlichen Jahrhundert stammenden alexandrinischen Musentempel (Musaion) des hellenistischen Königs Ptolemaios I. ${ }^{58}$ Beide stellen das Museum als eigenes Gebäude dar, als Ort der Gelehrsamkeit, des Zusammentreffens, -lebens und -arbeitens von Gelehrten. Im Hauptartikel vermerkt der Zedler zunächst nur, ein „Museum heisset [...] eine Kunst-Kammer, ein Müntz-Cabinet, Rarität- und Antiquitäten-Kammer“59 und geht erst in einem gesonderten Artikel auf das „Museum Ashmolean“ in Oxford ein. Es wird als ein „sehr merckwürdiges Gebäude“ dargestellt, in dem viele „,rare und sehenswürdige Sachen, als Alterthümer, Naturalien und andere von den besten Künstlern verfertigte Raritäten aufgehoben“60 würden. Dagegen integriert der Encyclopédie-Artikel das „musée ashmoléen“ quasi als Prototyp in den Hauptartikel:

Le mot de musée a reçu depuis un sens plus étendu, \& on l'applique aujourd'hui à tout endroit où sont renfermées des choses qui ont un rapport immédiat aux arts \& aux muses. [...] Le musée d’Oxford, appellé musée ashmoléen, est un grand bâtiment que l’Université a fait construire pour le progrès \& la perfection des différentes sciences. ${ }^{61}$

Anders als bei Zedler wird in der Encyclopédie interessanterweise nicht nur auf Grundbestand, Geschichte und Sammlungszugewinne eingegangen, sondern auch darauf, von wem die Sammlung geordnet wurde und wer sich als Donator hervorra-

55 Damit schließe ich mich der Vorgehensweise u.a.Claudia Valters und Stefan Siemers an. Vgl. VALTER, Kunst- und Naturaliensammlungen; SIEMER, Geselligkeit und Methode.

56 Eine systematische Analyse des Museumsbegriffs findet sich bei VALTER, Kunst- und Naturaliensammlungen, 28-38.

57 Diese Reflexion ist natürlich auch auf die anderen erläuterten Begriffe, insbesondere den des Naturalienkabinettes anzuwenden.

58 Weitere Informationen hierzu finden sich in Kapitel 2 der vorliegenden Arbeit.

59 ZEDLER, Bd 22, 1739, Sp. 1375.

60 ZEDLER, Bd 22, 1739, Sp. 1378.

61 Encyclopédie, Bd 10, 894. Ein Kurzeintrag zu musée findet sich außerdem in den, Tables Analytiques, Bd 2 (I-Z), 268. 
gender Sammlungsobjekte hervorgetan hat. ${ }^{62}$ Zwei Aspekte erscheinen hier besonders bedeutsam: Erstens bietet danach eine Institution Gelehrten verschiedener Disziplinen einen Raum für Austausch und ,zunftgerechten Lebenswandel’. So gesehen stellt ein Museum einen Ort des (Gelehrten-)Austausches, einen Kommunikationsraum dar. ${ }^{63}$ Zweitens ist die Darstellung des Ashmolean Museum als universitätszugehöriges Museum par excellence hervorzuheben, an dem sich zu messen sein wird.

Der Begriff Museum konnte durchaus auch auf schriftliche Dokumente, allen voran die den Sammlungen zugehörigen Kataloge, bezogen werden, ${ }^{64}$ wie es beispielsweise beim Museum Wormianum ${ }^{65}$ aus dem Jahr 1655 der Fall ist. ${ }^{66}$ Darüber hinaus entstanden im Laufe vor allem des ausgehenden 18. Jahrhunderts zahlreiche literarische Museen. Hierbei handelt es sich um Zeitschriften bzw. Periodika, die den Titel Museum im Namen führen und eine Art literarisches (Kuriositäten-)Kabinett darstellen. „Selbstverständlich finden [sic] in diesen literarischen Kunst- und Wunderkammern auch ein Teil der tatsächlich existierenden Kabinette Erwähnung. “67 Anhand der bis hierher wiedergegebenen Aussagen lassen sich schon einige der für die Entstehung eines Museums notwendigen Bedingungen erkennen: Raum in Form eines Gebäudes, hinreichende finanzielle Mittel und - modern gesprochen - Sponsoren.

Gegenüber den ausführlichen Beschreibungen der alten Lexika erscheint die allgemeine Museumsdefinition eines aktuellen Brockhaus erschreckend lapidar: „Museum [lat. ,Ort für gelehrte Beschäftigung’, von griech. museion ,Musensitz‘, zu mousa

62 Dort heißt es: „Dans le même tems [sic], Élie Ashmole, écuyer, fit présent à l’université d’Oxford d'une collection considerable de curiosités qui y furent acceptées, \& ensuite arrangées \& mises en ordre par le docteur Plott, qui fut établi premier garde du musée. Depuis ce tems [sic], cette collection a été considérablement augmentée, entr'autres d'un grand nombre d'hiéroglyphes, \& de diverses curiosités égyptiennes que donna le docteur Huntingdon [sic], d'une momie entiere donnée par M. Goodgear, d'un cabinet d'histoire naturelle dont M. Lister fit présent, \& de diverses antiquités romaines, comme autels, médailles, lampes, \&c.“ Encyclopédie, Bd 10, 894.

63 Diesen Aspekt macht vor allem STEFAN SIEMER in seiner mit „Sammeln als kommunikative Praxis“ überschriebenen Einleitung deutlich. Vgl. Ders., Geselligkeit und Methode 1-13, bes. 2 u. 3.

64 Vgl. VALTER, Kunst- und Naturaliensammlung, 33-35.

65 OlE WORM, Museum Wormianum seu historia rerum rariorum tam naturalium, quam Artificialium, tam Domesticarum, quam Exoticarum, quae Hafniae Danorum in aedibus Authoris servantur, Amstelodami 1655.

66 Als weitere bekannte Beispiele lassen sich. das Museum Hoffmanniani (1741) oder das Museum Schöpflini (1773) heranziehen. Vgl. VALTER, Kunst- und Naturaliensammlung, 132-141.

67 VALTER, Naturalienkabinette, 21-30, 23. 
,Muse'] [...], seit dem 18. Jh. öffentl. Sammlung von künstler. und wiss. Gegenständen und deren Gebäude.“68

\section{Naturalienkabinett}

Sich dem Begriff Naturalienkabinett über den Umweg Kabinett zu nähern, kommt dem gleich, was in Übersetzungsarbeiten häufig als ,false friends’ bezeichnet wird. Heißt es doch gleich im ersten Satz des entsprechenden Eintrags in der Encyclopédie: „Le mot cabinet doit être pris ici dans une acception bien différente de l'ordinaire“. ${ }^{69}$ Im Folgenden soll der Unterschied zwischen beiden verdeutlicht werden.

Das Kabinett wird als eine Art - durchaus repräsentativer - Rückzugsort dargestellt, das „kleiner als andere Zimmer gemacht [wird], weil es nur vor einzelne Personen gebauet wird.“" ${ }^{70}$ Dieser Ort wurde vom jeweiligen Hausherren genutzt, um in aller Abgeschiedenheit seinen Studien nachzugehen oder wertvolle Objekte (auch Kuriosita oder Naturalien) unterzubringen, und wurde allenfalls mit wenigen ausgewählten Personen geteilt, um z.B. geheime Besprechungen abzuhalten. Den Damen des Hauses habe das Kabinett als Ort „du recueillement \& de la solitude““71 gedient.

Hingegen heißt es zu den Räumlichkeiten eines Naturalienkabinettes:

un cabinet d'Histoire naturelle est ordinairement composé de plusieurs pieces \& ne peut être trop étendu; la plus grande salle ou plûtôt le plus grand appartement, ne seroit pas un espace trop grand pour contenir des collections en tout genre des différentes productions de la nature. ${ }^{72}$

Geradezu enthusiastisch wird dem geneigten Leser das Panorama eines solchen Kabinetts ausgebreitet:

en effet, quel immense \& merveilleux assemblage! comment même se faire une idée juste du spectacle que nous présenteroient toutes les sortes d'animaux, de végétaux, \& de minéraux, si elles étoient rassemblées dans un même lieu, \& vues, pour ainsi dire, d'un coup d'œil? ce tableau varié par des nuances à l'infini, ne peut être rendu par aucune autre expression, que par les objets mêmes dont il est composé: un cabinet d'Histoire naturelle est donc un abregé de la nature entiere. ${ }^{73}$

68 Brockhaus - Die Enzyklopädie: in 24 Bänden. 20. überarbeitete und aktualisierte Auflage, Bd 15, Leipzig - Mannheim 1998, 261.

69 Encyclopédie, Bd 2, 489.

70 ZEDLER, Bd 5, 1733, Sp. 16.

71 Encyclopédie, Bd 2, 488.

72 Encyclopédie, Bd 2, 489.

73 Encyclopédie, Bd 2, 489. 
Die Unterscheidung vollzieht sich jedoch nicht nur über die baulichen Differenzen, sondern auch über die Funktion und Nutzung ${ }^{74}$ des Kabinetts und die in ihm ausgeübten Praktiken. Im Zedler werden diese beiden Aspekte verknüpft. Dort heißt es: „Naturalien-Kammern, Naturalien-Cabineter, sind gewisse Gemächer, worinnen Naturalien gesammelt und aufbehalten werden. In solchen Gemächern muß eine gewisse Ordnung beobachtet werden, [...].“" ${ }^{75}$ Im weiteren Verlauf wird eine detaillierte Ordnung aufgestellt, an deren erster Stelle der Mensch an der Spitze der lebenden Geschöpfe und an deren Ende die Mineralien stehen. Zugleich werden immer auch Konservationsmethoden für die einzelnen Objekte genannt. Der Artikel schließt mit einer Übersicht über bekannte Beschreibungen von Raritäten- und Naturalienkabinetten. Ergänzend erfahren wir aus der Encyclopédie: „Mais un cabinet d'Histoire naturelle est fait pour instruire.“" ${ }^{76}$ Der weitaus umfangreichere Artikel der Encyclopédie stimmt inhaltlich im Großen und Ganzen mit dem des Zedler überein. Allerdings beinhaltet er eine ausführliche Beschreibung des dem Jardin du Roi zugeordneten Cabinet du Roi. Diese führt sowohl die Ordnung des Kabinetts als auch dessen Reichtum anschaulich vor Augen und lässt den Leser in dem Glauben zurück, dass kein anderes Naturalienkabinett mit dessen Vielfalt und Perfektion mithalten könne. ${ }^{77}$ Ebenso finden sich mehrseitige Anweisungen des als Experten herangezogenen M. Aubenton, des „garde \& démonstrateur du cabinet du Roi“, über die Ordnung und Instandhaltung sowie die angemessene Vorzeigepraxis der Objekte. ${ }^{78}$ Die beiden wichtigsten Aspekte von Naturalienkabinetten der Aufklärungszeit sind Ordnung ${ }^{79}$ und Unterrichtung.

Gegenüber dem Abriss im Zedler handelt es sich bei der Darstellung in der Encyclopédie darüber hinaus um einen deutlich provokativeren Text. So enthält der Artikel z.B. einen flammenden Aufruf gegen Personen, die ohne Geschmack und Geist

74 Natürlich vorbehaltlich der Einschränkung, dass die Anlage der Räumlichkeiten und die Art der Nutzung auch davon abhängen, ob man sich in einem Bürgerhaushalt oder dem ,appartement destiné à un très-grand seigneur“" (Encyclopédie, Bd 2, 488) befindet.

75 ZEDLER, Bd 23, 1740, Sp. 1231f.

76 Encyclopédie, Bd 2, 490.

77 Dies wird durch einen Verweis auf die hohen Besucherzahlen des Cabinet du Roi noch unterstrichen. Vgl. Encyclopédie, Bd 2, 490.

78 Vgl. Encyclopédie, Bd 2, 490-492.

79 In der Encyclopédie wird dies mit dem Satz „Cependant qu'est-ce qu'une collection d'êtres naturels sans le mérite de l'ordre?“ noch einmal auf den Punkt gebracht. Vgl. Encyclopédie, Bd 2, 490. 
sammelten und besser daran täten, ihre Sammlungen zu vernichten denn auf diese Art weiterzusammeln. ${ }^{80}$ Der Artikel schließt mit einem Plädoyer zur Errichtung eines Tempels der Natur, dessen äußere Erscheinungsform mit den Inhalten korrespondieren solle. ${ }^{81}$ Ein Entwurf zur modernen Museumsidee, wie sie im 19. Jahrhundert ihre Entfaltung fand.

Eine weitere Verwendung des Wortes Cabinet oder auch Cabinetgen bezeichnet einen „mit ausgelegter Mustir-Künstlich Bildhauer-Tisch und Drechsler-Arbeit gezierte[n] Schranck, worinne[n] man allerhand Kostbarkeiten, Natur- und Kunst-Sachen, sonderlich in denen antiquitæten-medaillen- und raritæten-Cammern verwahret“82 werden. Diese Bedeutung passt zwar in den Zusammenhang von Naturalienkabinetten, ist für diese Arbeit jedoch von untergeordneter Bedeutung.

Liest man die Lexikonartikel zu Museum und Naturalienkabinett direkt hintereinander, so erschließt sich auf den ersten Blick nicht, ob es inhaltliche Unterschiede zwischen den Beständen beider Einrichtungen gab und welche Kriterien für die Verwendung des jeweiligen Begriffs angelegt wurden. Die einzigen fassbaren Unterscheidungskriterien betreffen die Raumfrage und den Grad der Zugänglichkeit oder auch Öffentlichkeit der jeweiligen Einrichtung. Unklar ist, inwieweit die Verwendung von Begriffen wie Naturalienkabinett und Museum im 18. Jahrhundert bewusst auf bestimmte Praktiken des Sammelns oder des Austausches verweisen. Ebenso wenig wird klar, inwieweit eine der beiden Einrichtungen einen höheren Stellenwert genoss. Eine eindeutige Abgrenzung zwischen Museum und Kabinett ist allein anhand der zu Rate gezogenen Lexika kaum möglich. ${ }^{83}$ Vielleicht ist eine begriffliche Abgrenzung an dieser Stelle aber auch nur bedingt sinnvoll, denn es stellt sich die Frage, ob hier nicht ein heutiger Geist versucht, eine Unterscheidung vorzunehmen, wo der aufklärerische eine Einheit annahm oder zumindest keinen Widerspruch sah. ${ }^{84}$

80 Vgl. Encyclopédie, Bd 2, 490.

81 Encyclopédie, Bd 2, 492.

82 ZEDLER, Bd 5, 1733, Sp. 16.

83 Auf diese Problematik wird in Bezug auf die Namensgebung des Academischen Museums detaillierter eingegangen. Vgl. Kapitel 2.2.5.

84 So ist bspw. 1805 in D. Johann Georg Krünitz’s ökonomisch-technologischer Encyclopädie zu lesen, dass ein Museum eine Sammlung von Kunstwerken, öfters auch Naturprodukten beinhalte. Vgl. D. JOHANN GEORG KRÜNITZ's ökonomisch-technologische Encyclopädie, oder allgemeines System der Staats-, Stadt-, Haus- und Landwirtschaft und der Kunstgeschichte, in alphabetischer Ordnung, Bd 98, Berlin 1805, 449-524, 449. 


\section{Naturgeschichte}

Ursprünglich auf Aristoteles zurückgehend, bezeichnet der Begriff Naturgeschichte an der Wende vom 18. zum 19. Jahrhundert das „Verzeichniß und die Beschreibung der natürlichen oder zu den drey Naturreichen gehörigen Körper“. ${ }^{85}$ Mit den drei Naturreichen oder auch Hauptklassen, die damit angesprochen werden, sind „das Thierreich, das Pflanzenreich und Mineralreich“" ${ }^{86}$ gemeint. Entsprechend handelt es sich bei Naturalien um „alle von der Natur selbst zusammen gesetzten Körper auf unserm Erdboden, so lange sie durch die Kunst noch nicht merklich verändert worden [...]. Daher das Naturalien-Cabinett, wo dergleichen Körper aufgestellet werden.“87 Bleibt noch anzumerken, dass die Naturgeschichte eine eigene wissenschaftliche Disziplin ${ }^{88}$ war, der in Göttingen ein starkes Interesse entgegengebracht wurde. ${ }^{89}$ So fanden dort unter Büttner bereits 1755 naturgeschichtliche Vorlesungen statt. Dieser war außerdem „der erste Gelehrte, der an deutschen Universitäten Naturgeschichte als selbständiges Kolleg las.“ ${ }^{90}$ Wesentliche Methode der Naturgeschichte war die vergleichende Beobachtung. In dem hier untersuchten Zeitraum gewann die Verzeitlichung von Taxonomien ${ }^{91}$ zunehmend an Bedeutung.

85 Adelung, Bd 3, Sp. 445; STichweH, Schmeizels (1728) paraphrasierend unterscheidet ,Historia Naturalis’, ,Historia sacra' (,zur Religion gehörige Dinge'); ,Historia Artificialis’ (,Dinge, die die Kunst hervorbringt'); und ,Historia Civilis’ (,im bürgerlichen Leben paßirte Dinge'). In: Ders, Zur Entstehung des modernen Systems wissenschaftlicher Disziplinen. Physik in Deutschland 17401890, Frankfurt a.M. 1984, 23 FN 49. ADELUNG, Bd 3, Sp. 449.

87 AdELUNG, Bd 3, Sp. 444.

88 Die Naturgeschichte stand immer auch in enger Verbindung zur Naturphilosophie. Für den Untersuchungszeitraum sind als wichtige Vertreter im 18. Jh. v. a. Kant, später Hegel, Schelling und Oken zu nennen.

89 Eine ausführliche Einführung in die Naturgeschichte gibt z. B. JOHANN FRIEDRICH BLUMENBACH, Handbuch der Naturgeschichte, Göttingen 1779. Dieses Handbuch wurde in verschiedene Sprachen übersetzt. Es erlebte insgesamt zwölf, immer wieder erweiterte und ergänzte Auflagen. Die letzte, von 1830 stammende Ausgabe ist auch online verfügbar unter: http://gdz-srv3.sub.unigoettingen.de/cache/toc/D110377.html (Zugriff am 1. Januar 2005).

90 PLISCHKE, Ethnographische Sammlung, 11, FN 5.

91 Bei dem Wort Taxonomie handelt es sich insofern um einen problematischen Begriff, als er heute von Wissenschaftlern verschiedener Disziplinen in unterschiedlichen Kontexten und mit veränderten Bedeutungsnuancen verwendet wird. Während er von Naturwissenschaftlern, insbesondere Biologen, im Sinne einer Einordnung von Lebewesen in ein biologisches System begriffen wird, rekurrieren Geisteswissenschaftler bei seiner Verwendung häufig auf die Linguistik. In der Sprachwissenschaft stellte die Taxonomie ein Teilgebiet dar, auf dem man durch Segmentierung und Klassifikation sprachlicher Einheiten den Aufbau eines Sprachsystems beschreiben will. Für den hier behandelten Zusammenhang ist die naturwissenschaftliche Auslegung maßgeblich. 


\section{Sammlung}

Soweit lexikalisch erschließbar kommt dem Ausdruck Sammlung im 18. Jahrhundert in Bezug auf Kabinette und Museen nur eine geringe Bedeutung zu. So führt Zedler zwar den Begriff „Sam[m]lung“ auf - allerdings nur mit der für unseren Zweck irrelevanten Bedeutung der Erb-Verbrüderung ${ }^{92}$. Darüber hinaus kennt er die „Sammlung von Natur- und Medicin- wie auch gehörigen Kunst- uns Literaturgeschichten“ als „gelehrtes Tagebuch, so im Jahr 1717 von einigen Breßlauischen Medicis unter der Direktion Joh. Ranolds [...] ist angefangen worden“. ${ }^{93}$ Der Begriff sammeln wird hier unter Rückgriff auf die Bibel erläutert. Unter den aufgeführten Bedeutungen findet sich auch eine, die für uns von Interesse ist, nämlich „[i]ngleichen etwas, das man hoch und werth hält, mit großer Sorgfalt zusammen bringen und aufheben, daß es nicht verderbe“ ${ }^{94}$. Unter Sammler ${ }^{95}$ wird jedoch lediglich die Tätigkeit eines Lumpensammlers beschrieben. In der Encyclopédie findet sich bezeichnenderweise weder ein Eintrag zu collection noch zu collectionneur. Erst der Adelung führt unter Sammlung auf: „Eine Menge mehrerer einzeln und nach und nach zusammen gebrachter Dinge. [...] Eine Sammlung machen. Die Büchersammlung, Kupfersammlung, Münzsammlung, Naturalien-Sammlung, u.s.f. Eine Sammlung von Reisen, von Kupfern, von Münzen.“96 Demnach handelt es sich bei Sammlung in Bezug auf die Fragestellung dieser Arbeit im Vergleich zu Kabinett und Museum um eine vergleichsweise neutrale Bezeichnung. In der vorliegenden Arbeit wird der Begriff im Sinne Pomians verstanden. Demnach ist eine Sammlung:

jede Zusammenstellung natürlicher oder künstlicher Gegenstände, die zeitweise oder endgültig aus dem Kreislauf ökonomischer Tätigkeiten herausgehalten werden, und zwar an einem abgeschlossenen, eigens zu diesem Zweck eingerichteten Ort, an dem die Gegenstände ausgestellt werden und angesehen werden können. ${ }^{97}$

92 Vgl. ZEDLER, Bd 33 (1742), Sp. 1697.

93 Beide Zitate aus ZEDLER, Bd 23 (1740), Sp. 1168.

94 Vgl. ZEDLER, Bd 33 (1742), Sp. 1701.

95 Vgl. ZEDLER, Bd 33 (1742), Sp. 1702.

96 ADELUNG, Bd 3 (1811), Sp. 1270.

97 Krzysztof Pomian, Zwischen Sichtbarem und Unsichtbarem: Die Sammlung, in: Ders. (Hg.), Der Ursprung des Museums. Vom Sammeln, Berlin 1988, 13-72, 16. Vgl. ebd. zu den Einschränkungen und Implikationen, die mit dieser Definition verbunden sind. 


\section{Wissenschaft}

Versucht man, das heutige Wissenschaftsverständnis schlagwortartig zu erfassen, erhält man als Kondensat Begriffe wie Vorurteilsfreiheit, Methodik, Wertfreiheit, Verifizierbarkeit, Möglichkeit der Kritik und Intersubjektivität. Wie verhält es sich aber mit der Wissenschaftsauffassung in der Spätaufklärung und frühen Romantik? Es liegt auf der Hand, dass die Frage was Wissenschaft um 1800 eigentlich ist hier nicht letztgültig beantwortet werden kann, da es sich dabei um ein eigenes, ausgedehntes Forschungsfeld handelt. ${ }^{98}$

Um zunächst zu einer Grunddefinition des Begriffes zu kommen, sei auf das Wörterbuch Adelungs zurückgegriffen. Dieses unterscheidet drei Bedeutungen des Wissenschaftsbegriffs. Erstens Wissenschaft als „Zustand, da man etwas weiß, Kenntniß, Nachricht davon hat“ und zweitens als „Inbegriff dessen, was man im engern Verstande weiß, der Inbegriff der klaren und deutlichen Begriffe, welche man hat, besonders die Einsicht in den Zusammenhang allgemeiner Begriffe“. ${ }^{99}$ Beiden Begriffen bescheinigt er, langsam zu veralten - dennoch sei hier auf sie hingewiesen, denn man trifft sie in den Quellen des ausgehenden 18. und beginnenden 19. Jahrhunderts noch häufig in diesen Bedeutungen an. Die letzte Definition wendet sich stärker dem zu, was wir heute als Wissenschaft verstehen und deutet bereits auf einen hinter der Definition stehenden wissenschaftstheoretischen Diskurs hin. Sie kennzeichnet Wissenschaft als
[o]bjective, von dem Inbegriffe in einander gegründeter allgemeiner Wahrheiten; wo- durch sich die Wissenschaft von der Kunst unterscheidet, indem diese bloß Ausübungs- sätze, jene aber in einander gegründete allgemeine Wahrheiten enthält. Es gibt demnach so viele Wissenschaften, als allgemeine Wahrheiten, wie Wahrheiten einer Art, und in einander gegründet betrachtet werden. ${ }^{100}$

Doch wie nähert man sich über eine solche Definition hinaus einem abstrakten Begriff wie Wissenschaft an? Wie erhält man eine Antwort auf die Frage: Wie funktioniert Wissenschaft zu dieser Zeit? Bezogen auf den Titel Sammeln für die Wissen-

98 Sofern die Frage, was Wissenschaft ist, nicht den Kern einer Untersuchung ausmacht, wird der Wissenschaftsbegriff oft unreflektiert verwendet oder der Bedeutungszusammenhang nicht näher eingeschränkt. Selbst Kuhn verzichtet in seiner berühmten Studie über die Struktur wissenschaftlicher Revolutionen auf eine Klärung des Wissenschaftsbegriffs und setzt mit einer Definition über normale Wissenschaft an. KuHN, Struktur.

99 Beide Zitate aus ADELUNG, Bd 3 (1811), Sp. 1582.

100 AdELUNG, Bd 3 (1811), Sp. 1582. 
schaft? sind vor allem wissenschaftliche Praktiken ${ }^{101}$ von Belang. Ein Rückgriff auf Zedler und die Encyclopédie erscheint in diesem Zusammenhang nur bedingt sinnvoll; denn dem Begriff Wissenschaft kommt im ausgehenden 18. Jahrhundert eine vielschichte Bedeutung zu. Dies spiegelt sich augenfällig in dem Umfang, den im Zedler der Komplex Wissen - Wissenschaft - Wissenschaften einnimmt wieder; er füllt 187 Spalten. $^{102}$ Dieses Kapitel ist erwartungsgemäß mehrfach untergliedert. Besonderen Raum nimmt gleich zu Anfang die Erläuterung einiger Stellen der Bibel ein, in denen Konjugationen des Wortes wissen erscheinen. Weitere Schwerpunkte bilden die Kapitel „Vergnügen, daß man aus der Wissenschafft schöpfft“ (Sp. 135995), „Art und Weise, wie man zur Wissenschafft gelangen könne“ (Sp. 1433-43), „Anwendung und Application der erlernten Wissenschafften“ (Sp. 1449-61) und das Kapitel „Aufnehmen und Wachstum derer Wissenschafften von Zeit zu Zeit“, das von der „Sündfluth“ bis ins 18. Jahrhundert reicht und mit folgendem Ausblick schließt: „Wissenschafften, ob dieselben ewig sind?“ (Sp. 1464-1517). Auch wenn einige Passagen schon eine Zugewandtheit zur Aufklärung erkennen lassen, insbesondere ist hier auf das Kapitel „Unterscheid [sic] der Wissenschafft von der Erfahrung“ (Sp. 1352-55) und den Ansatz, dass bei den Wissenschaften „auf deren Nützlichkeit zu sehen“ (1443f) sei, zu verweisen, bleibt grundsätzlich ein theologischmetaphysisch verortetes, und in Teilen teleologisches Argumentationsmuster gewahrt.

Ganz anders in der Encyclopédie: Inhaltlich werden darin weitgehend gleiche Themenbereiche abgedeckt, doch gewinnt der Wissenschaftsbegriff für die Encyclopédie noch einmal eine qualitativ gesteigerte Komponente. Noch stärker als bei anderen Begriffen tritt hier der programmatisch aufklärerische Gestus in den Vordergrund. Der Einfluss, der diesem Begriff beigemessen wird, lässt sich bereits daran erkennen, dass er den wesentlichen Teil des Discours préliminaire d'Alemberts einnimmt. ${ }^{103}$ Die (progressivsten) Encyclopädisten entwickelten ein eigenes wissenschaftstheoretisches Konzept, das stark in den Zusammenhang von Produktivkräften und Ökono-

101 Diesem Ansatz folgt beispielsweise der von Helmut Zedelmaier und Martin Mulsow herausgegebene Sammelband Die Praktiken der Gelehrsamkeit in der Frühen Neuzeit.

102 Davon entfallen ca. sieben Spalten auf Wissen, ca. 52 auf Wissenschaft und ca. 128 auf Wissenschaften. ZEDLER, Bd 57.

103 Vgl. JeAn Le Rond D'AlEmBert, Einleitung zur Enzyklopädie. Durchgesehen und mit einer Einleitung herausgegeben von Günther Mensching, Hamburg 1997. 
mie eingebunden war und nach dem „alle wissenschaftliche Erkenntnis einen praktisch-politischen Zweck“ habe. ${ }^{104}$ Grundton dieser Theorie ist eine vom Deduktionismus abgewandte, subjektorientierte Wissenschaftsauffassung. Sie zielt darauf ab, eine Einheit der Wissenschaften herzustellen, durch deren gebündelte Kräfte eine „Befreiung der Menschen von den Zwängen der unerkannten Natur“ als „Voraussetzung politischer Emanzipation“ und wirtschaftlichen Fortschritts denkbar würde. ${ }^{105}$ So wird dem Begriff Wissenschaft übersteigerte Bedeutung zugewiesen.

Neben wissenschaftstheoretischen Ansätzen finden sich in den genannten Lexika auch immer wieder Hinweise, wie man Wissen erwerben könne, so z.B. „1. durch Bücher, 2. durch eigenes Nachsinnen, 3. durch Unterrichtung und 4. durch Erfahrung“; ${ }^{106}$ doch lohnt es sich, einmal darüber nachzudenken, wie sich eine Annäherung an wissenschaftliche Praktiken noch vollziehen ließe. Hier ist zunächst die Frage zu stellen, welche Quellen darüber Aussagen erlauben. In welchem Zusammenhang wurden diese Quellen erzeugt, und natürlich: Welche Informationen geben sie preis, was lässt sich folgern? Wen akzeptiert man als verlässlichen Berichterstatter für die jeweilige Fragestellung? Welche Praktiken zeichnet die frühmoderne Wissenschaft aus, welche Methoden sind ihr eigen? Welcher Organisationsstruktur folgt Wissenschaft, welches sind die Orte, an denen sie ausgeübt wird? Wer ist an der Produktion von Wissen beteiligt? Welche Rolle spielen geographischer, sozialer und kultureller Ort der Akteure? Welcher Stellenwert wird der Religion bzw. der Ausübung religiöser Praxis beigemessen? Was ist das eigentliche Erkenntnisinteresse, welche inhaltlichen Schwerpunkte werden gesetzt?

Hier sind für das ausgehende 18. Jahrhundert vor allem zu nennen: Reiseberichte, Abhandlungen in gelehrten Zeitschriften, Briefwechsel unter Gelehrten, Aufstellungssystematiken von Sammlungen, die uns eine Grundvorstellung der damals als maßgeblich angesehenen Ordnungen vermitteln und nicht zuletzt auch die Monographien führender Wissenschaftler, die sich zu Grundlagenwerken entwickelten. In zunehmendem Maße gewinnen auch Instrumente und zugehörige MessAufzeichnungen für die historische Forschung an Bedeutung. In jüngster Zeit wurden

104 Günther Mensching, Die Enzyklopädie und das Subjekt der Geschichte, in: Jean Le Rond d'Alembert, Einleitung zur Enzyklopädie. Durchgesehen und mit einer Einleitung herausgegeben von Günther Mensching, Hamburg 1997, XVI-XLIX, XVII.

105 Beide Zitate aus MEnSCHING, Die Enzyklopädie, XVI-XLIX, XV, XIX.

106 ZEDLER, Bd 57 (1748), Sp. 1436. 
zudem verstärkt außeruniversitäre, oder allgemeiner: außerinstitutionelle Praktiken der Gelehrsamkeit und die zugehörigen Quellen in den Blick genommen, die u.a. die Familie als Beteiligte und als sozialen Ort der Wissensproduktion einbeziehen. ${ }^{107}$

Anhand solcher Quellen lassen sich einige grundlegende Tendenzen aufzeigen. So wird schnell klar, dass die Gewinnung von Wissen nicht nur ergebnisorientiert, sondern auch und vor allem prozessorientiert angelegt ist. Das Erproben vor allem neuer Verfahrenswege wird ausführlich beschrieben, die zugehörigen Zwischenergebnisse werden mitgeteilt. ${ }^{108}$ Eine immens wichtige Rolle spielen darüber hinaus Ordnungen und Ordnungssysteme, wie bereits in dem Abriss über die Naturalienkabinette deutlich geworden ist. Hier ergeben sich weiterführende Fragen wie: Welche Ordnungen wurden in welchen Zusammenhängen erstellt? Wer waren ihre Urheber? Wie erfuhren sie Verbreitung, und von wem wurden sie mit welchen Abweichungen rezipiert? An dieser Stelle ist ausdrücklich auf das Sammeln oder präziser: bestimmte Arten des Sammelns und Klassifizierens als wissenschaftliche Praktiken hinzuweisen. Was geschah mit den Objekten, nachdem sie den ökonomischen Kreisläufen entzogen worden waren um in Sammlungen eingegliedert zu werden? Wurden sie ihrerseits aktiv in das Schaffen von Erkenntnis eingebunden? Der hohe Stellenwert der Ordnungen legt nahe, dass der Reihenfolge, in der Handlungen zu geschehen hatten, ebenfalls eine gewisse Bedeutung beigemessen wurde. Darüber hinaus lässt die in der Encyclopédie in Bezug auf Naturalienkabinette geäußerte Kritik an Personen, die ohne Geist und Geschmack ${ }^{109}$ sammelten, auf die Existenz eines gewissen verbindlichen Wertesystems schließen. Doch wie wird dieses Wertesystem eingeübt, wie wird Wissen vermittelt und was gilt als Wissenskanon, was als erlernenswerte Metho-

107 In diesem Zusammenhang ist auf den auf Heide Wunder zurückgehenden Begriff des Arbeitspaares hinzuweisen. Vgl. HeIDE Wunder, „Er ist die Sonn’, sie ist der Mond“: Frauen in der Frühen Neuzeit, München 1992. Dieser Begriff wird bspw. verwendet von MoNIKA MOMMERTZ, Schattenökonomie der Wissenschaft. Geschlechterordnung und Arbeitssysteme in der Astronomie der Berliner Akademie der Wissenschaften im 18. Jahrhundert, in: Theresa Wobbe (Hg.), Frauen in Akademie und Wissenschaft: Arbeitsorte und Forschungspraktiken 1700-2000, Berlin 2002, 3163.

108 Hier soll exemplarisch auf die von Vertretern verschiedener Fachrichtungen, v. a. Chemie und Medizin, gemeinsam unternommene Untersuchung der dem Academischen Museum vom dänischen König geschenkten Mumie verwiesen werden. Vgl. UAG Kur 4 V g 8 und GGA vom 24. Februar 1781, 185f). Eine ausführliche Beschreibung der unternommenen Versuche findet sich in: GGA vom 11. Juni 1781, 569-572 und GGA vom 8. Oktober 1781, $985 f$.

109 Vgl. Encyclopédie, Bd 2, 490. 
de ${ }^{110}$ Hier geraten Hauslehrer und Gymnasien, Kavalierstouren und Gelehrtenreisen, Universitäten und Akademien und vor allem immer wieder ausgedehnte Briefwechsel in den Blick. Besonders letztere verweisen unmittelbar auf ein weiteres konstitutives Element der (früh-) neuzeitlichen Gelehrtengesellschaft: die Ausbildung (wissenschaftlicher) Netzwerke über Landes- und auch gewisse Standesgrenzen hinweg. Dieser Gedankengang darf aber nicht dazu verleiten, von einem - im heutigen Sinne - egalitären System gleichberechtigter Partnerschaften auszugehen. Den angesprochenen Briefwechseln wohnt eine ex post nicht leicht zu durchschauende Hierarchiestruktur inne, die überdies mit einem komplexen System des Gabentausches verknüpft ist. Auch an der Universität selbst spielten Hierarchien eine wichtige Rolle. Bereits Stichweh verwies auf die Relevanz von Hierarchien für die frühmoderne Wissenschaftsorganisation, in der Disziplinen(grenzen) noch eine untergeordnete Rolle spielen. Er zeigt im Wesentlichen zwei Arten von Hierarchien auf: eine „Hierarchie der Wissensformen“ und eine „Hierarchie der Fakultäten“. ${ }^{111}$

Nachdem kurz angerissen worden ist, wie man sich dem Aufbau der Wissenschaften nähern kann, stellt sich die Frage, was eigentlich ihr Erkenntnisinteresse war. Eine auf den ersten Blick etwas lapidare, bei genauerer Überlegung jedoch recht tiefsinnige Antwort liefert Goethe, wenn er Faust wünschen lässt, „daß ich erkenne, was die Welt im Innersten zusammenhält. “112 Denn diese Antwort beinhaltet sowohl eine greifbar materielle als auch eine metaphysische Komponente. Sie schließt den Wunsch nach dem Erkennen von Ordnungssystemen und Wertsystemen ebenso ein wie die Suche nach stofflichen Zusammensetzungen oder mechanischen Prinzipien. In einem weiter gefassten Kontext wäre hier auf die Komplementarität von Natur und Kultur, Kunst und Wissenschaft einzugehen.

Mit diesen Fragen ist die Vielschichtigkeit des Wissenschaftsbegriffs bei weitem nicht ausgeschöpft. Doch ist diese Untersuchung nicht unmittelbar auf das Wesen von Wissenschaft ausgerichtet, so dass das Fragenspektrum an dieser Stelle nicht weiter aufgefächert werden soll. Sinnvoller erscheint hier eine Fokussierung auf die für die Fragestellung der vorliegenden Arbeit relevanten Aspekte. Als Kondensat der

${ }^{110}$ Hier ist bspw. an spezifische Formen des Sehens und des Beobachtens sowie an Experimentierund Messpraxis zu denken.

111 STICHWEH, Wissenschaftliche Disziplinen, 15.

112 Johann Wolfgang von Goethe, Faust. Eine Tragödie. I. und II. Teil. Ungekürzte Ausgabe, München 1956, Erster Teil, 25. 
aktuellen Forschung lässt sich dafür folgendes Resümee ziehen: Wesentliches Element der Wissenschaftspraxis des ausgehenden 18 . und beginnenden 19. Jahrhunderts sind kommunikative Prozesse. Wissenschaftlicher Austausch vollzieht sich dabei vor allem in Briefwechseln, im Publizieren von Zeitschriften oder so auch in Göttingen - in Diskussionen bei Akademiesitzungen ${ }^{113}$ und in gelehrten Abendgesellschaften. ${ }^{114}$ Bei der Ausbildung und Ausweitung kommunikativer Netzwerke spielten Freundschaften oder auch persönliche Streitigkeiten keine geringe Rolle. Die wissenschaftliche Methodik begann, sich mit dem Aufkommen einzelner Fachdisziplinen auszudifferenzieren. Waren zunächst noch Beobachtung, Beschreibung und Komparatistik vorherrschende Merkmale wissenschaftlicher Praktiken, so gewannen (naturwissenschaftliche) Experimente zunehmend an Bedeutung. ${ }^{115}$ Damit veränderten sich auch die Orte der Wissensproduktion. Noch ist es zu früh, von Laboratorien zu sprechen, treffender ist vielleicht der Begriff Beobachtungsstationen, was z.B. Observatorien mit einschließt. Charakteristisch ist ebenso eine zunehmende Institutionalisierung, v.a. im Bereich der Medizin. Immer häufiger entstanden Fachkliniken mit speziell zugeschnittenen Präparatesammlungen. Kennzeichnend für die Wissenschaft des ausgehenden 18. Jahrhunderts ist eine Umbruchsituation, die sich auch durch die Suche nach neuen Leitwissenschaften auszeichnet. Allerdings ist zu berücksichtigen, dass sich die Einzeldisziplinen erst am Beginn ihres Ausdifferenzierungsprozesses befanden.

Welche Arbeitsweisen als wissenschaftlich gelten können, hängt vom jeweiligen Untersuchungsgegenstand und der auf ihn angewandten Methode (z.B. chemische Untersuchung gegenüber Komparatistik) ab. Und doch lässt sich fach- und methodenübergreifend grundsätzlich eine Art Dreischritt ausmachen: Erstens Durchführung der Untersuchung und genaues Beobachten, zweitens Mitteilung von Untersuchungsmethode, Beobachtungen und Schlussfolgerungen. Dabei ist die Angabe von

113 Hier ist auf die Relevanz von Institutionen hinzuweisen, die mit ihrer Infrastruktur oftmals gelehrte Diskussionen ermöglichten oder förderten.

114 Ein schönes Beispiel hierfür gibt ein Besuch Georg Forsters in Göttingen: „Am 21. Januar 1779 war Georg Forster zum ersten Mal in Göttingen. Lichtenberg und Sprengel veranstalteten zu Ehren Forsters ein Essen, bei dem der damals fünfundzwanzigjährige Professor an der Ritterakademie zu Cassel auf dem Ehrenplatz zwischen dem Rektor der Universität und Heyne saß. An dem Souper nahmen neben anderen teil Büttner, Gmelin, Blumenbach und auch Meiners..." PlischKE, Ethnographische Sammlung, 22.

115 Hier deutet sich bereits ein Übergang von qualitativ zu quantitativ orientierter Wissenschaft an. 
Referenzen, wie verwendeter Literatur oder Expertenauskünften anhand von Briefen an einzelne Gelehrte oder durch Aufsätze, Vorträge oder Vorführung an eine größere Gruppe, ein wichtiges Element. Drittens Überprüfung durch andere, Annahme der Ergebnisse oder kontroverse Diskussion. 


\section{$2 \quad$ Sammeln im Großen und im Kleinen}

\subsection{Zur Geschichte des Sammelns}

Fragt man heute verschiedene Personen nach ihrer spontanen Assoziation zu dem Wort Museum, so berichten sie meistens von der beeindruckenden, das Stadtbild vieler europäischer Metropolen prägenden Architektur der Museumsbauten - Zeugnisse der großen Welle der Museumsgründungen im ausgehenden 19. Jahrhundert. Vor allem naturhistorische und ethnologische Museen entstanden damals in großer Zahl. Die Tradition des Sammelns, Bewahrens und Präsentierens ist jedoch erheblich älter.

Der Beginn der Museumsgeschichte wird - sowohl begrifflich als auch inhaltlich meist auf den schon erwähnten Alexandrinischen Musentempel König Ptolemaios I. (3. Jh. v. Chr.), das so genannte Museum Alexandrinum, zurückgeführt. Es gilt, modern gesprochen, als Bildungsanstalt, in der sowohl Kunstwerke und wissenschaftliche ${ }^{116}$ Instrumente als auch Bücher zusammengebracht und zur Erkenntnisgewinnung herangezogen wurden. Im Vordergrund stand hier nicht nur eine systematische, interessengelenkte Besitzanhäufung und Forschung, sondern vor allem die Nutzung des Museums als Kommunikationsort der Gelehrten untereinander sowie zwischen Gelehrten und Schülern. Allerdings handelte es sich hierbei in keiner Weise um eine öffentliche Bildungseinrichtung im heutigen Sinn, sondern um einen Ort, dessen Zutritt nur Privilegierten gestattet und dessen Lehrbetrieb auf Auserwählte beschränkt war.

Erste überlieferte Beschreibungen von Sammlungen existieren ebenfalls bereits aus der Zeit des antiken Griechenlands. ${ }^{117}$ Aus dem Mittelalter sind Sammlungsinventare von Schatzkammern geistlicher wie weltlicher Fürsten überliefert. Zu dieser Zeit war das Sammeln stark auf den sakralen Bereich ausgerichtet. Insbesondere Reliquien, liturgische Gegenstände aus kostbaren Materialien und Kunstschätze ${ }^{118}$ wurden gesammelt. Als besonders begehrenswert galten außerdem „Edel- und Halbedelsteine

\footnotetext{
${ }^{116}$ Hier in der Bedeutung von Erkenntnis bringend bzw. Erkenntnis versprechend.

117 SCHLOSSER, Kunst- und Wunderkammern, 5, erwähnt hier insbesondere PAUSANIAS (ca. $110 \mathrm{n}$. Chr.-180 n. Chr.), Beschreibung Griechenlands (10 Bde).

118 Hier ist beispielsweise an Gemälde, illuminierte Handschriften oder Wandbehänge zu denken.
} 
[...] wegen ihrer Schutzwirkungen gegen Gift, als Antidota gegen Krankheiten und sonstige Übel“. ${ }^{119}$ Solch eine Konnotation haftete auch diversen kuriosen Naturalia und Exotika an, deren Existenz in den Sammlungskammern wesentlich auf den (Aber-)Glauben an ihre heilbringende Wirkung zurückzuführen ist. Schlosser verzeichnet für das Spätmittelalter zahlreiche solcher Sammlungsgegenstände und folgert besonders in Bezug auf kirchliche Sammlungen: „In diesen Kirchenschätzen des ausgehenden deutschen Mittelalters ist der Charakter der späteren Kunst- und Wunderkammern schon kenntlich angelegt““. ${ }^{120}$ Kostbare Materialien einerseits und religiös verorteter Wunderglaube andererseits bemaßen den Wert der Sammlungsgegenstände. Die mittelalterlichen Sammlungen kirchlicher wie weltlicher Fürsten dienten vornehmlich repräsentativen Zwecken, der Zurschaustellung von Macht und Besitz. Doch Schlosser hebt noch einen weiteren Aspekt kirchlicher Sammlungen hervor, den des Zeigens und Belehrens:

[D]iese von der Kirche unter ihren mächtigen Schutz genommenen und gleichsam geheiligten Dinge [waren] unter bestimmten Bedingungen und Beschränkungen öffentlich zugänglich [...]. In eigenen Tribünen, hier und da auch in eigenen festen Gebäuden ausgestellt, wurden sie bei feierlichen Gelegenheiten dem Volke öffentlich vorgezeigt und erklärt. $^{121}$

Vom 15. Jahrhundert an ließ sich die „Ausbreitung von Privatsammlungen modernen Typs“ beobachten: ${ }^{122}$ Die legendären „Kunst- und Wunderkammern der Spätrenaissance“ entstanden. ${ }^{123}$ Sie können bereits für die Mitte des 16 . Jahrhunderts als in Europa vorherrschender Sammlungstyp betrachtet werden. Diese Kammern zeichneten sich vor allem durch eine Vielfalt und einen Facettenreichtum an Sammlungsstücken aus, die heutigen Betrachtern häufig als systemlos, ja chaotisch erscheinen. Dabei gab es ein gewisses Einvernehmen darüber, was sammelwürdig sei - trotz Unterschieden in Umfang und Ausstattung der jeweiligen Kabinette. Einige Objekte galten für Kabinette, deren Besitzer auf ihre Reputation bedacht waren, als Standardrepertoire. ${ }^{124}$ Wundertiere, Gegenstände aus kostbaren Materialien, Seltenheiten von Rei-

119 SCHLOSSER, Kunst- und Wunderkammern, 14.

120 SCHLOSSER, Kunst- und Wunderkammern, 20. Vgl. auch Pomian, Die Sammlung, 32.

121 SCHLOSSER, Kunst- und Wunderkammern, 18.

122 POMIAN, Vom Sammeln, 9.

123 SCHLOSSER, Kunst- und Wunderkammern, Titel.

124 An der Spitze rangierte z. B. lange Zeit das Horn des Narwals, das als Auswuchs des fabelhaften Einhorns angesehen wurde. Es erschien nicht nur wegen seiner Seltenheit, sondern auch wegen der ihm zugeschriebenen heilenden Eigenschaften als besonders begehrenswert. 
sen in ferne Länder, aber auch alltägliche Dinge, die berühmte Vorbesitzer hatten, ${ }^{125}$ fanden ihren Weg in diese Kabinette. Hier wird bereits ersichtlich, dass Objekte nicht nur wegen ihres materiellen, sondern auch auf Grund ihres symbolischen Wertes gesammelt wurden. Pomian bezeichnet sie als Semiophoren, als „Gegenstände, die das Unsichtbare repräsentieren, das heißt, die mit einer Bedeutung versehen sind“ und die durch ihre materialen Eigenschaften als Mittler zwischen Sichtbarem und Unsichtbarem fungieren. ${ }^{126}$ Eine ungefähre Vorstellung einer solchen - allerdings vor allem mit Naturalien ausgestatteten - Wunderkammer vermittelt der, nachstehend abgebildete, berühmte Stich vom Kabinett des neapolitanischen Apothekers Ferrante Imperato (1521-1609).

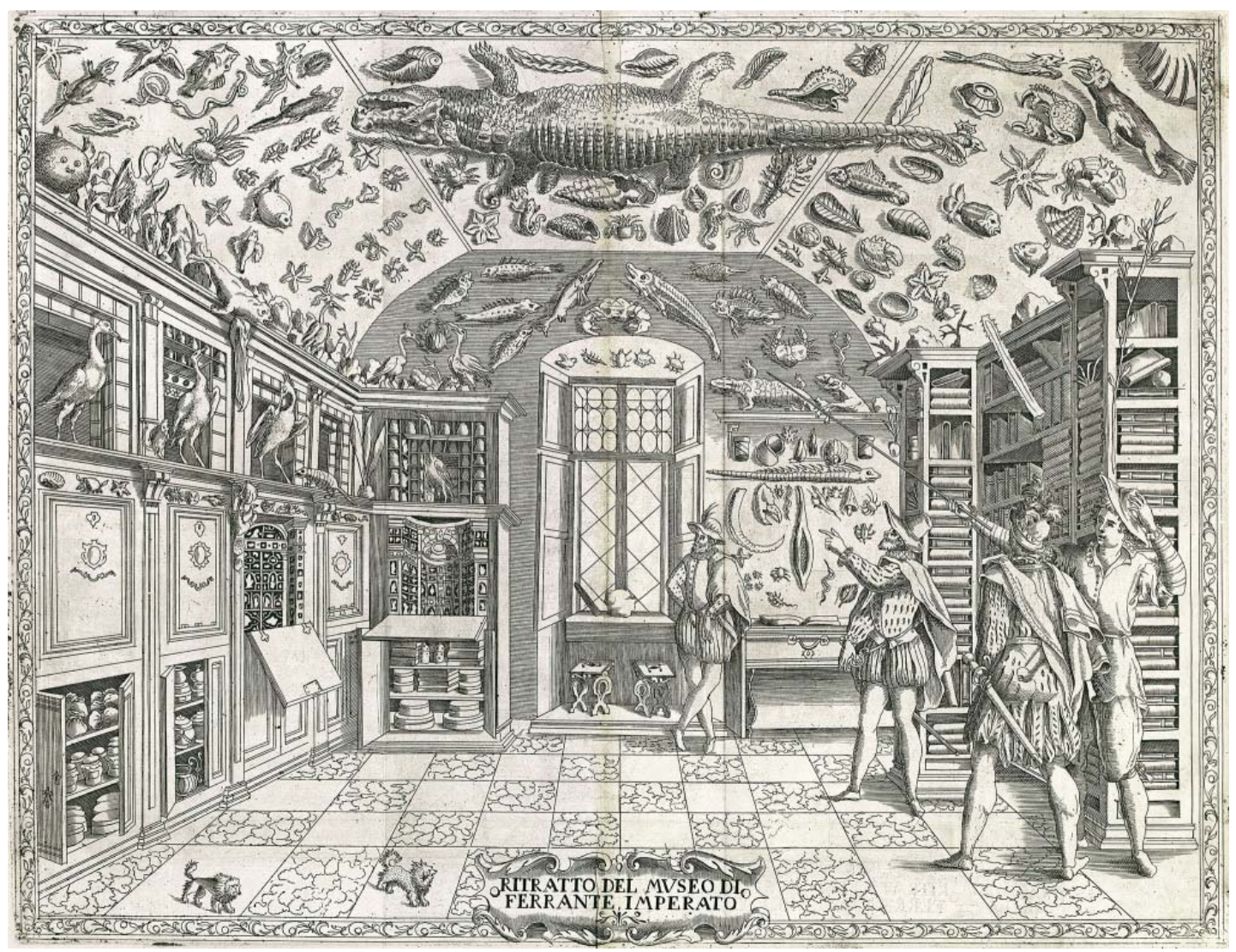

Abbildung 1: Naturalienkabinett des neapolitanischen Apothekers Ferrante Imperato (um 1599)

${ }^{125}$ Heyl führt als Beispiel den Hosenlatz Heinrichs VIII. an. Vgl. CHRISTOPH HEYL, Einhorn und Indianermantel, in: Spektrum der Wissenschaft Spezial: Frühe Neuzeit. Forschung und Technik in der Renaissance 4 (2004), 12-15, 14.

126 POMIAN, Die Sammlung, 13-72, 50. 
Neben diesen am Objekt orientierten, aber weit über dessen materiale Eigenschaften hinausgehenden Wunderglauben, war die Bedeutung der Gegenstände im Rahmen eines übergeordneten Ganzen ein wesentliches Element der Kunst- und Wunderkammern der Spätrenaissance. Dies fand seinen Ausdruck in dem Wunsch, in den Sammlungen ein Abbild der Welt, der Erde und des Himmels, gleichsam einen „Macrocosmos in Microcosmo“ “127 zu schaffen. ${ }^{128}$ In diesem Bestreben gründet der enzyklopädische Charakter der manieristischen Sammlungen. Ein jedes Ding sollte in diesen Kammern einen Repräsentanten finden. So ist die „Kunstkammer als ein in sich geordnetes Ganzes zu verstehen, dessen scheinbar disparate Glieder die kosmische Ordnung des Universums widerspiegeln.“129 Als sinnfälliger Ausdruck dessen entstand im 16. Jahrhundert die Bezeichnung „theatrum mundi“ (Welttheater) in „Abwandlung der von Psalm 24 getroffenen Definition der Erde ,als Kunstkammer Gottes'“. ${ }^{130}$ Somit sind die Kunst- und Wunderkammern gleichermaßen Ausdruck von Selbstdarstellung, Neugierde und Weltverständnis ihrer Besitzer, wie auch des Wunsches, die sich - in der Sichtweise der Zeitgenossen - durch vermehrte Reisetätigkeit stets vergrößernde Welt zu erfassen. ${ }^{131}$ Und doch fand sich in diesen Kammern selten das Alltägliche, schnell Greifbare, sondern vor allem das Außergewöhnliche und Seltene. Von Lorraine Daston stammt die Beobachtung, dass darin 99,9 Prozent des Kosmos zugunsten des Singulären und Anormalen irgnoriert wurden. “132

Die Initiatoren dieser Kammern waren oftmals weltliche Herrscher von hohem Rang und Ansehen, die über eine gute Ausbildung und so in gewisser Hinsicht selbst über enzyklopädisches Wissen verfügten. Macht und Reichtum ermöglichten es ihnen, ihrer Sammelleidenschaft nachzugehen und deren Ergebnisse für repräsentative Zwecke zu nutzen oder auch einzelne Objekte - im Sinne des frühneuzeitlichen Ga-

${ }^{127}$ Vgl. hier den programmatischen Titel des von ANDREAS GROTE herausgegebenen Sammelbandes Macrocosmos in Microcosmo. Die Welt in der Stube. Zur Geschichte des Sammelns 1450 bis 1800, Opladen 1994.

${ }^{128}$ Kritisch zu dieser Auslegung des Mikrokosmos-Begriffs: LORRAine DASTON/KATHERINE PARK, Wunder der Kunst, Wunder der Natur, in: Dies. (Hg.), Wunder und die Ordnung der Natur 11501750, Frankfurt a.M. 2002, 301-354, 320f.

129 SCHEICHER, Kunst- und Wunderkammern der Habsburger, 12.

130 Vgl. hierzu SCHEICHER, Kunst- und Wunderkammern der Habsburger, 12.

131 Entdeckungsfahrten und später Forschungsreisen bilden ein wesentliches Element für die Ausweitung der Sammlungsbestände und deren Ordnungen.

132 Vgl. LORRAINE J. DASTON, The factual sensibility, in: Isis 79 (1988), 452-470, 458. 
bentausches - in den diplomatischen Verkehr einzubeziehen. Auch diese Sammlungen wurden nicht im großen Stil vorgeführt, sondern blieben ausgewählten Besuchern vorbehalten. Den Zweck dieser Kuriositätenkabinette allein mit Repräsentationsansprüchen oder der Darstellung von Herrschaft über die Welt im Kleinen wie im Großen zu erklären, greift zu kurz. Sie waren vielmehr auch „Zeugen der individuellen curiositas ihrer Besitzer, die sich auf verschiedene Wissenschaften und Künste kaprizierten“. ${ }^{133}$ Insofern dienten sie der Ergötzung und Erbauung, aber eben auch Studienzwecken. Gerade neuere Untersuchungen verweisen auf die durchaus wissenschaftlich motivierte Neugierde bzw. das Erkenntnisstreben der Initiatoren. ${ }^{134}$ So gab es sehr wohl das Bestreben, durch die Sammlungen einen Grundstock zur Erforschung der Natur und ihrer Geheimnisse im weitesten Sinne zu legen - wenn auch mit einem anderen Verständnis von Wissenschaft als in späteren Jahrhunderten. ${ }^{135}$ Ein wichtiges Element hierfür waren die Orte der Sammlungen, genauer ihre Platzierung an Herrscherhöfen. Diese Residenzen waren nicht nur Macht- und Repräsentationszentren, sondern auch Zentren der Wissensproduktion, an denen Forscher, Alchimisten, Künstler, Naturkundler, Ärzte etc. zusammenkamen und an denen die Möglichkeit zu gelehrtem Austausch bestand.

Für das 16. und 17. Jahrhundert sind bereits in verhältnismäßig großer Zahl Quellen zu berühmten Sammlungen überliefert, ${ }^{136}$ so dass die Geschichte des Sammelns häufig als Geschichte einzelner Sammlungen erscheint. Als prominente Sammler traten vor allem Mitglieder des Adels und Hochadels in Erscheinung. Doch auch kirchliche Fürsten standen weiterhin als Sammlerpersönlichkeiten im Vordergrund. Besondere Erwähnung verdient die so genannte Ambraser Sammlung ${ }^{137}$ Erzherzog Ferdinands II. von Tirol (1529-1595), eine der bedeutendsten europäischen Kunstsammlungen ihrer Zeit, ${ }^{138}$ die später in den Besitz seines Neffen Rudolf II. überging und

133 VALTER, Kunst- und Naturalienkabinette, 21-30, 22.

134 Hier sei exemplarisch verwiesen auf VALTER, Kunst- und Naturalienkabinette, bes. 11f.

135 Zum Wissenschaftsbegriff im ausgehenden 18. und beginnenden 19. Jahrhundert vgl. auch Kap. 1 der vorliegenden Arbeit.

136 Eine beeindruckende Auflistung verschiedener Quellengruppen findet sich bei PoMIAN, Vom Sammeln, 9.

137 Vgl. SCHEICHER, Kunst- und Wunderkammern der Habsburger, 73-136. Hier vermitteln zahlreiche Abbildungen einen Einblick in den Reichtum dieser Sammlung.

138 Die Tatsache, dass Kunstsammlungen in diesem Kapitel nur geringe Aufmerksamkeit finden, liegt in der Fragestellung der vorliegenden Arbeit begründet. Sie darf aber nicht darüber hinwegtäuschen, dass Kunstschätze, soweit zurückverfolgbar, für die gesamte Geschichte des Sammelns eine 
dessen Prager Kunstkammer erweiterte. ${ }^{139}$ Daneben ist die von Kurfürst August von Sachsen (1526-1586) angelegte Kunstkammer des Dresdener Hofes zu erwähnen. Sie bildete den Grundstock für die späteren vielfältigen, insbesondere durch August II. (1670-1733) stark geförderten Sammlungen. ${ }^{140}$ Im Laufe des 17. Jahrhunderts entstanden zudem vermehrt Kabinette in bürgerlichen Kreisen. Insbesondere Apotheker und Ärzte verfügten oftmals über derartige Kabinette, da sie im Rahmen ihrer Berufsausübung häufig mit sammlungswürdigen Objekten in Berührung kamen.

Grobes Ordnungskriterium der Kunst- und Wunderkammern war die Unterteilung in Naturalia, also der Natur entnommene, unbearbeitete Gegenstände und Artificialia, also von Menschenhand geschaffene oder in irgendeiner Form bearbeitete Gegenstände. ${ }^{141}$ Die Feinstrukturierung konnte dagegen stark variieren. Pyenson und Sheets-Pyenson gehen so weit zu formulieren: „There were probably as many different classification schemes as there were individual collectors“. ${ }^{142}$ Dennoch lassen sich hier einige Strömungen oder Hauptvarianten unterscheiden. Eine Anordnung nach ästhetischen Gesichtspunkten, also nach Form, Farbe, Material oder Größe gilt als am stärksten verbreitet. Diese darf aber nicht allein als Hilfslösung aufgrund fehlender einheitlicher Taxonomien und sonstiger Ordnungssysteme angesehen werden, sondern muss ebenso als Zeugnis einer stark ausgeprägten visuellen Kultur verstanden werden. Eine zweite Gruppe „related to the four elements, where rocks, plants, and insects, represented earth; fish represented water; birds represented air; and arti-

konstitutive Rolle gespielt haben, wenngleich zeitweise andere Objekte wie z. B. Münzen oder eben Kuriosita größere Aufmerksamkeit genossen. Dabei ist der Begriff Kunstsammlung in seiner historischen Dimension zu verstehen. Vgl. hierzu das vorangegangene Kapitel, insbes. den Aspekt Differenzierung zwischen Museen und Naturalienkabinetten. Zur Geschichte von Kunstmuseen vgl. z.B. JAmES J. SheEHAN, Geschichte der deutschen Kunstmuseen. Von der fürstlichen Kunstkammer zur modernen Sammlung, München 2002.

139 Insgesamt sind die habsburgischen Sammlungen heute wissenschaftshistorisch von großem Wert, denn sie zeichnen sich durch eine „gute Überlieferung und Katalogisierung [aus], die jeweils testamentarisch verfügt war.“ NADINE KuBE, Ausstellung als Medium (unveröffentlichte Magisterarbeit), Göttingen 2004, 11.

${ }^{140}$ Eine ausführliche Darstellung dieser Sammlungen findet sich bei BECKER, Vom RaritätenKabinett zur Sammlung. Dort insbes. Kapitel II.1 „Palais des Sciences“ und „Cabinet d’Ignorance“. Sammeln und Ordnen am Dresdner Hof - der inszenierte Besitz , 44-66.

141 Als Beispiele für Naturalia seien genannt: Mineralien und Korallen, Konchylien, getrocknete Insekten und Pflanzen, Tierhäute und Schildkrötenpanzer. Unter Artificialia fielen - in dieser groben Strukturierung - goldverzierte Vasen aus Halbedelsteinen, Fayencen und Uhren ebenso wie mit Miniatur-Schnitzereien verzierte Kirschkerne und gedrechselte Elfenbeinkugeln.

142 LeWIS Pyenson/Susan SheEts-Pyenson, Showing: Museums, in: Dies. (Hg.), Servants of nature: A history of scientific institutions, enterprises and sensibilities, London 1999, 125-149, 127. 
ficial productions like glassware or tools represented fire.“" ${ }^{143}$ Als letzte Gruppe trat die bereits aus dem vorigen Kapitel bekannte Dreiteilung auf, die die einzelnen Objekte den drei Reichen der Natur zuordnet, unterteilt in Tiere, Pflanzen und Mineralien. Diese Art der Aufstellung gewann mit Einsetzen der Frühaufklärung an Bedeutung und wird uns - in erweiterter Form - im Academischen Museum in Göttingen wieder begegnen.

Etwa um das Jahr 1700 lässt sich ein Wandel beobachten. Verfeinerte Klassifikationssysteme und vor allem das Erstarken der empirischen Wissenschaften führen zu einer Ablösung der Kunst- und Wunderkammern durch Kunst- und Naturalienkammern. ${ }^{144}$ Dabei veränderte sich nicht unbedingt der Inhalt der Sammlungen, sondern vielmehr die Anordnung der Objekte, die langfristig zu einer Spezialisierung der Sammlungen auf einzelne Objektbereiche führte. Hier zeigt sich der Einfluss der Frühaufklärung. Als einflussreicher Vertreter ist vor allem Leibniz zu nennen, mit dem das spezialisierte enzyklopädische Sammeln noch einmal einen Aufschwung erlebte. Irmgard Müsch zeigt eine weitere, etwas früher einsetzende Nuance dieses Wandels auf. Sie weist, ausgehend von der Eröffnung des bereits erwähnten Oxforder Ashmolean Museums, auf eine gesamteuropäische Entwicklung hin, die den sukzessiven Umbau fürstlicher Sammlungen mit primär repräsentativen Aufgaben zu wissenschaftlich geordneten und öffentlich zugänglichen Bildungsinstitutionen mit volkswirtschaftlichem Nutzen einleitete. ${ }^{145}$ Dies lässt sich anhand der (Er-)Öffnung einiger ,großer Häuser’ belegen. In erster Linie ist hier das Ashmolean Museum zu nennen, dessen spätere Sammlung bereits um 1630, damals noch als Kuriositätenkabinett der Familie Tradescent „gegen ein kleines Entgelt“146 jedermann zugänglich war. 1683 öffnete es als erstes eigentliches Museum seine Pforten. ${ }^{147} 1709$ folgte in Kassel durch Landgraf Karl von Hessen-Kassel die Angliederung des Kunsthauses, des so genannten Ottoneums, an das neu gegründete Collegium Carolinum, mit dem

143 Pyenson/ Sheets-Pyenson, Showing: Museums, 127.

144 Valter verstärkt diese These noch: „Spätestens zu Beginn des 18. Jahrhunderts ist die Sammlungsform der Kunst- und Naturalienkammer europaweit fest etabliert.“ VALTER, Kunst- und Naturalienkabinette, 23.

145 IRMGARD MÜSCH, Nicht nur der Neugier allein, 61.

146 HEYL, Einhorn und Indianermantel, 15.

147 Ausführlich hierzu vgl. ArThur MacGregor, Ark to Ashmolean. The story of the Tradescants, Ashmole and the Ashmolean Museum, Oxford 1983; ferner: R.F. OvenELL, The Ashmolean Museum, 1683-1894, Oxford 1986. 
Zweck, ,seine Sammlungen - unabhängig vom Hof - einer breiteren Nutzung zuzuführen“. ${ }^{148}$ In St. Petersburg waren vom Jahr 1719 ab die Sammlungen Peters des Großen zugänglich. Zeitgleich ließ er ein eigenes Gebäude für diese Sammlungen errichten. 1754 wurde die Herzogliche Kunst- und Naturalienkammer, die Vorläuferin des heutigen Herzog Anton Ulrich-Museums in Braunschweig einer breiteren Öffentlichkeit zugänglich gemacht und 1759 öffnete das British Museum seine Pforten. Allerdings darf ,Zugang für eine breitere Öffentlichkeit' nicht mit ,freiem, unreglementierten Zugang' verwechselt werden. Strukturell wurde versucht, durch die Festlegung von Öffnungszeiten, das Erlassen und Verkünden von Besucherordnungen, sowie die Vergabe von Eintrittskarten den Besucherstrom zu lenken. ${ }^{149}$ Darüber hinaus genossen durch Stand oder Profession privilegierte Besucher in der Regel deutlich größere Rechte und erhielten auch intensivere Betreuung als Fremde und Interessierte ohne entsprechende Reputation.

Der Wunsch, Sammlungen einem größeren Publikum zugänglich zu machen, ließ sich nicht immer mit der vorhandenen Infrastruktur vereinbaren. Dies war sicherlich einer der Gründe dafür, dass

[f]ürstliche Naturalienkabinette [...] nach 1750 mehrfach Universitäten zugewiesen [wurden]; neben Bayreuth ging z.B. die Sammlung des Herzogs von Mecklenburg-Schwerin 1790 an die Universität Rostock [...], die Sammlung von Franz Ludwig von Erthal an die Universität Bamberg. [...] Die Kgl. Kunstkammer in Berlin wurde 1798 der Akademie der Wissenschaften unterstellt [...]. ${ }^{150}$

Das Göttinger Museum schließt sich in gewisser Hinsicht diesem Trend an, doch entstand es nicht aus der Schenkung einer fürstlichen Sammlung, sondern durch den Ankauf der Privatsammlung eines Professors der Georgia Augusta, die dieser bereits zuvor in seine Lehrtätigkeit einbezogen hatte. ${ }^{151}$ Bürgerliche Kabinette hatten im Laufe des 17. Jahrhunderts zunehmende Verbreitung erfahren und avancierten in der ersten Hälfte des 18. Jahrhunderts „zu Orten regen wissenschaftlichen Austauschs,

148 MüsCH, Nicht nur der Neugier allein, 61.

149 Für das Beispiel Braunschweig sei in diesem Zusammenhang auf folgenden Aufsatz hingewiesen: Oliver MATUSCHEK, „Hunde mitzubringen verbeten“. Besucher und Besucherbetreuung im Kunst- und Naturalienkabinett, in: Kunstmuseum des Landes Niedersachsen Herzog Anton UlrichMuseum Braunschweig (Hg.), 250 Jahre Museum. Von den fürstlichen Sammlungen zum Museum der Aufklärung. Ausstellung in der Burg Dankwarderode, Braunschweig, 29. April bis 22. August 2004, München 2004, 88-99.

150 MÜSCH, Nicht nur der Neugier allein, 67f, FN 9.

151 So heißt es bspw. bereits in den GGA vom 12. April 1756, 377: „Die Natur-Geschichte lehret Herr Commißarius Büttner täglich zwey Mahl, Vormittags und Nachmittags, und weiset zugleich sein Cabinet vor.“ 
und kaum ein Gelehrter verzichtete auf das Anlegen einer eigenen, häufig spezialisierten Sammlung“. ${ }^{152}$ Der Bestand dieser Sammlungen war in der Regel deutlich kleiner als derjenige fürstlicher Sammlungen, unterlag dafür jedoch meist einer regelmäßigen Nutzung, so dass hier der Ort war, an dem Ordnungssysteme entwickelt und erprobt, und an dem zuerst Gebrauchskataloge erstellt wurden. Gerade in Bezug auf die „Bestandserfassung und -systematisierung mittels eines gedruckten Kataloges “153 schreibt Valter den bürgerlichen Sammlungen eine initiative Rolle zu.

Die zunehmende Etablierung von Sammlungen spiegelt sich in der zeitgenössischen Literatur wie auch in im Sammlungskontext auftretenden (neuen) Quellengruppen wider. Hier sind v.a. Sammlungskataloge, zunächst als Inventare, später als wissenschaftliche Hilfsmittel und letztlich auch als Auktionskataloge zu nennen. Eine gewisse Vorbildfunktion für den Katalog als wissenschaftliches Hilfsmittel nahm hier der - posthum erschienene - Katalog des dänischen Arztes Ole Worm ein. Dessen Museum Wormianum ${ }^{154}$ von 1655 erlangte durch eine bisher nicht dagewesene „Ausführlichkeit der Klassifizierung und Kommentierung der Objekte““55 einen hohen Bekanntheitsgrad. Dieses Werk wurde elf Jahre später durch Olearius, ${ }^{156}$ den damaligen Aufseher der Gottorfischen Kunstkammer

[h]insichtlich der Anzahl und Aktualität der vor allem bei den Naturalien angeführten Literaturhinweise [noch übertroffen]. Letztere belegen die Funktion der Kataloge als wissenschaftliches Hilfsmittel: begleitet von einer qualitätvollen Illustrierung waren die hier angeführten Objekte wichtige Grundlage zur Identifizierung der Spezies, da eine einheitliche Nomenklatur noch fehlte. ${ }^{157}$

Hinzu traten steigende Zahlen von Reisebeschreibungen, in denen sich vermehrt Beschreibungen einzelner Kabinette finden. Eine weitere wichtige Gattung stellen museologische bzw. museumstheoretische Schriften dar. Neben der Frage, was denn eigentlich zu sammeln sei, gehen sie vor allem von der Frage der richtigen Ordnung und Anordnung der Objekte nach. Verhältnismäßig früh lassen sich sammlungstheoretische Schriften nachweisen. Als die ältesten gelten die im Jahr „1565 von dem

152 BECKER, Vom Raritäten-Kabinett zur Sammlung, 91.

153 VALTER (2004), 22.

154 OLE WORM, Museum Wormianum seu historia rerum rariorum tam naturalium, quam Artificialium, tam Domesticarum, quam Exoticarum, quae Hafniae Danorum in aedibus Authoris servantur, Amstelodami 1655.

155 VALTER, Kunst- und Naturalienkabinette, 22.

156 ADAM OlEARIUS, Gottorfische Kunstkammer, worinnen Allerhand ungemeine Sachen, so theils die Natur, theils künstliche Hände hervorgebracht und bereitet, Schleswig 1666.

157 VALTER, Kunst- und Naturalienkabinette, 22. 
Münchener Arzt und Sammlungsverwalter Herzog Albrechts V., Samuel Quiccheberg (1529-1567)““158 herausgegebenen Inscriptiones vel tituli theatri amplissimi. ${ }^{159}$ Hierbei handelte es sich um ein recht kompliziertes theoretisches Ordnungsmodell, das keine praktische Umsetzung erfuhr. ${ }^{160}$ Allerdings greift Daniel Wilhelm Moller rund 150 Jahre nach Quiccebergs Veröffentlichung bei der Abfassung seiner eigenen sammlungstheoretischen Schrift, Commentatio de technophysiothameis sive germanice von Kunst- und Naturalienkammern aus dem Jahr 1704 auf Quiccheberg zurück. Diese Schrift zeichnet sich ebenfalls dadurch aus, dass sie zwar „eine Bestandsaufnahme theoretischer Positionen ist, daß sie in der Praxis des Sammelns und Ordnens aber zunächst wenig Wirkung zeigte.“161 Eine deutlich größere Rezeption erfuhren hingegen die Schriften Johann Daniel Majors ${ }^{162}$, Michael Bernhard Valentinis ${ }^{163}$ und Caspar Friedrich Einckels ${ }^{164}$. Auch in die „populärwissenschaftliche Unterhaltungsliteratur“'165 fanden Kabinette nach und nach Eingang. ${ }^{166}$

158 VALTER, Kunst- und Naturalienkabinette, 22.

159 SAMUEL VON QUiCCHEBERG, Inscriptiones vel tituli theatri amplissimi, complectentis rerum universitatis singulas materias et imagines eximias, ut idem recte quodque dici possit. Promptuarium artificiosarum miracolosarumque rerum, ac omnis raris thesauri et pretiosa supellectilis, structurae atque picturae quae hic simulin theatro conquiri consaluntur, ut eorum frequenti inspectione tractatione, singularis alique rerum cognito et prudentia admiranda, citò, facilé ac tutò comparari possit, Monachii 1565.

160 Vgl. BECKER, Vom Raritäten-Kabinett zur Sammlung, 23, 26.

161 BECKER, Vom Raritäten-Kabinett zur Sammlung, 26.

162 Major publizierte mehrere Schriften, die sich mit Kunst- und Naturaliensammlungen auseinandersetzten. Die wohl berühmteste unter ihnen ist JOHANN DANIEL MAJOR, Unvorgreiffliches Bedencken von Kunst- und Naturalien-Kammern insgemein, Kiel 1674.

163 Michael Bernhard Valentini, Museum Museorum, Oder Vollständige Schau-Bühne Aller Materialien und Specereÿen Nebst deren Natürlichen Beschreibung, Election, Nutzen und Gebrauch, Aus andern Material-Kunst- und Naturalien-Kammern, Oost- und West-Indischen Reißbeschreibungen, Curiosen Zeit- und Tag-Registern, Natur- und Artzney-Kündigern, wie auch selbst-eigenen Erfahrung. Zum Vorschub Der Studirenden Jugend, Materialisten, Apothecker und deren Visitatoren, Wie auch anderer Künstler, als Jubelirer, Mahler, Färber, u.s.w. also verfasset, und Mit etlich hundert sauberen Kupfferstücken Unter Augen geleget Von D. Michael Bernhard Valentini, Franckfurt am Mayn 1704.

164 CASPAR FRIEDRICH EINCKEL, Museographia oder Anleitung zum rechten Begriff und nützlicher Anlegung der Museorum, oder Raritäten-Kammern, Leipzig - Breslau 1727, zu den verschiedenen Schreibweisen des Autorennamens vgl. BECKER, Vom Raritäten-Kabinett zur Sammlung, 92, der zudem eine ausführliche Vorstellung der genannten Schriften bietet.

165 VALTER, Kunst- und Naturalienkabinette, 23.

166 Als Beispiele führt VALTER, Kunst- und Naturalienkabinette, 23 an: „Eberhard Werner Happels Grösste Denkwürdigkeiten der Welt oder So genandte Relationes Curiosae von 1687 und Carl Friedrich Paullinis Philosophische Lust-Stunden (1706)“. 
Insgesamt lässt sich folgende Tendenz aufzeigen: Im Laufe des 18. Jahrhunderts wurden Naturalienkabinette vermehrt der Öffentlichkeit zugänglich gemacht. ${ }^{167}$ In der Regel handelte es sich dabei um fürstliche oder herrschaftliche Sammlungen, die aus der Tradition der Kunst- und Wunderkammern erwachsen waren und unter dem Einfluss der Frühaufklärung eine bestimmte Transformation erfuhren: Nicht nur die Art der gesammelten Objekte veränderte sich, sondern auch der Umgang mit ihnen. ${ }^{168}$ Claudia Valter fasst dies prägnant zusammen:

\begin{abstract}
Neben die repräsentative Funktion fürstlicher Sammlungen traten pädagogische und ökonomische Aufgaben. Die symbolische Ordnung und kosmologische Ausrichtung barocker Kunstkammern verblaßte zugunsten eines naturwissenschaftlich begründeten Programms [...]. An die Stelle des Sammelns bedeutsamer Einzelstücke trat die systematische Erschließung der ganzen Natur. Viele fürstliche Sammlungen blieben im Ganzen weiterhin enzyklopädisch, doch erfolgte eine Spezialisierung im inneren Aufbau, besonders was die Einteilung in Naturalia und Artificialia betraf. ${ }^{169}$
\end{abstract}

Wichtige Impulse für die Weiterentwicklung von Ordnungen und Sammlungstypen gingen dabei von bürgerlichen Privatsammlungen aus. Mitte des 18. Jahrhunderts vollzog sich ein Wandel von fürstlichen und bürgerlichen Privatsammlungen zu öffentlich zugänglichen Museen. Während um 1780 die Sammlung gewissermaßen als Institution etabliert war, ${ }^{170}$ befanden sich die Museen erst am Anfang eines Institutionalisierungsprozesses: Die mit veränderten Zugangs- und Nutzungsbedingungen einhergehenden spezifischen Strukturen eines Museums begannen sich langsam herauszubilden. An dieser Stelle setzt die Entstehung des Academischen Museums Göttingen ein.

167 Der Begriff öffentlich findet, soweit nicht explizit anders ausgewiesen, in dieser Arbeit in einer recht offenen Form Verwendung und meint: nicht von vornherein auf einen (näher) bestimmten Personenkreis eingeschränkt.

168 Welchen Bedeutungswandel Objekte erfahren können, zeichnet Pomian anhand der Vasensammlung der Medici eindrucksvoll nach. KRZYsztof Pomian, Für eine Geschichte der Semiophoren. Anmerkungen zu den Vasen der Medici-Sammlungen, in: Ders. (Hg.), Der Ursprung des Museums. Vom Sammeln, Berlin 1988, 73-90.

169 MüsCH, Nicht nur der Neugier allein, 61.

170 Vgl BECKER, Vom Raritäten-Kabinett zur Sammlung, Vorwort. 


\subsection{Das Academische Museum Göttingen - Sammlung und Institution in der Zeit der Gründung und Entwicklung}

\subsubsection{Vom Wunsch zur Wirklichkeit: Erwerbungsgeschichte}

Der Gedanke, in der Göttinger Universität eine Naturaliensammlung oder zumindest eine Naturalienkammer unterzubringen, geht noch in die Zeit vor der Georgia Augusta zurück. So liegen bereits aus dem Jahr 1733 „Pläne des Klosterbaumeisters Joseph Schaedeler für den Umbau des als Gymnasium Illustre dienenden ehemaligen Dominikanerklosters zum Universitätsgebäude“ vor. Im Grundriss der zweiten Etage

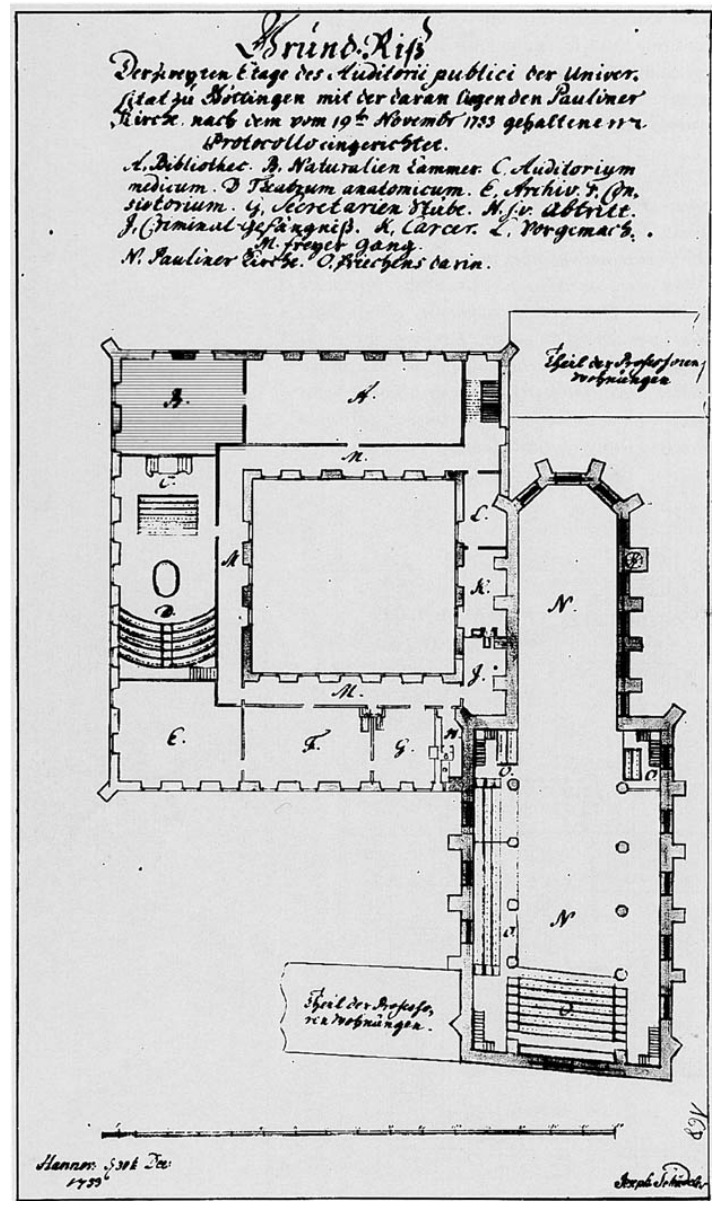

Abbildung 2: Grundriss der zweiten Etage des späteren Universitätsgebäudes (1733) ist, wie in Abbildung 2 erkennbar, Raum für eine „Naturalien Cammer“ vorgesehen. ${ }^{171}$

Bis zum Ankauf einer Naturaliensammlung für die Universität sollten allerdings noch vierzig Jahre vergehen. Erste Belege für den Wunsch nach der Gründung einer wissenschaftlichen Sammlung oder genauer: eine einer wissenschaftlichen Institution zuzuordnenden Sammlung in Göttingen lassen sich in Akten aus dem Jahr 1756 nachweisen. Aus einem Schreiben des Kameralisten Johann Heinrich Gottlob von Justi ${ }^{172}$ vom 17. Juni 1756 an den „Hochgebiethende[n] HErr[n] Geheimte[n] Rath und Cammer President[en]“ in Hannover ist zu erfahren, dass ihm ein Naturalienkabinett zum Kauf angeboten

171 Beide Zitate aus REIMAR ECK, Vom Pädagogium zur Keimzelle von Universität und Bibliothek. Zur Bau- und Nutzungsgeschichte des Pauliner-Klosters im 18. Jahrhundert, in: ELMAR MitTLER (Hg.), 700 Jahre Pauliner Kirche: vom Kloster zur Bibliothek. Ausstellung in der Niedersächsischen Staats- und Universitätsbibliothek Göttingen, 10. Dezember 1994-15. Januar 1995, Göttingen 1994, 145-149 und 160-163 (Katalogteil), 160.

172 Johann Heinrich Gottlob von Justi (1720-1771) lebte zwischen 1755 und 1757 in Göttingen und hatte die Position eines „Bergrathes und Oberpolizeicommissairs mit Berechtigung zu Vorlesun- 
worden sei, das „aus einem Beträchtlichen Vorrathe aus allen dreÿ Natur Reichen Bestehet und wobeÿ eine Sammlung von 400 Stück Zur Naturlichen Geschichte gehörigen Büchern mit verkaufet werden sollen.“" ${ }^{173}$ Justi selbst hatte an diesem Kabinett allerdings kein Interesse, da sich seine „Samlung bloß auf Foßilien erstrecke, die Bereits eben so gut ist, als daß Mineral Reich in diesem Cabinette“. ${ }^{174}$ Allerdings bescheinigte er:

\begin{abstract}
Allein es wäre Zu wünschen, daß Mittel ausfindig gemacht werden könten dieses Cabinet vor die Societaet der Wißenschaften zu acquiriren; und Ew: Hochfreÿherrl. Excellentz würden dadurch Dero ewig Prë̈ßwürdigen Anstalten und Stiftungen einen neüen Glanz geben. Eine Societat der Wißenschaften, deren Bemühungen hauptsächlich auf die natürliche Geschichte gerichtet seÿn müßen, hat ein Naturalien Cabinet fast unumgänglich nöthig. ${ }^{175}$
\end{abstract}

Obwohl Justi einiges an Überredungskunst aufbot, um den Kammer-Präsidenten davon zu überzeugen, und obwohl er mit Prestigegewinn und Notwendigkeit argumentierte und der Überzeugung Ausdruck verlieh, dass man den geforderten Preis von 1500 Rthlr vermutlich noch um 300 Rthlr drücken könne, erhielt er aus Hannover einen abschlägigen Bescheid. Im Antwortschreiben vom 19. Juni 1756 heißt es, dass Justis gute Absicht durchaus anerkannt und auch dessen Meinung über den Nutzen eines solchen Kabinetts geteilt werde, jedoch „die Umstande der Casse so enge Schrancken haben[,] daß dergleichen acquisitiones nicht zu machen - sondern nur zu wünschen sind.“ ${ }^{176}$

Im Jahr 1759 wurde erneut die Anschaffung eines Naturalienkabinettes für die Universität erwogen. Dabei bleibt offen, von wem dieses Mal die Initiative ausging. Indirekt lässt sich erschließen, dass von Seiten der Regierung in Hannover für die Anschaffung einer nicht näher bezeichneten Sammlung die Summe von 2000 Reichstalern zur Verfügung gestellt werden sollte. Ein Verzeichnis dieser Sammlung ist erhalten. Allerdings sprach sich Gesner, ${ }^{177}$ damaliger Bibliotheksdirektor und klassischer Philologe, nach Beratungen mit zwei weiteren Professoren, Büttner und dem

gen an der Universität“ inne. (THEODOR INAMA vON STERNEGG, Johann Heinrich Gottlob von Justi , in: ADB 14, 747-753, 748). Er lehrte Staatsökonomie und Naturgeschichte und war selbst Besitzer eines Naturalienkabinettes.

173 Beide Zitate aus UAG Kur 4 V g 1, Schreiben vom 17. Juni 1756.

${ }^{174}$ UAG Kur 4 V g 1, Schreiben vom 17.06.1756 an den „Hochwohlgebohrne[n] Freÿherr[n] Hochgebiethende[n] HErr[n] Geheimte[n] Rath und Cammer President[en] Gnädige[n] HErr[n].

175 UAG Kur 4 V g 1, Schreiben vom 17.06.1756 an den „Hochwohlgebohrne[n] Freÿherr[n] Hochgebiethende[n] HErr[n] Geheimte[n] Rath und Cammer President[en] Gnädige[n] HErr[n].

176 UAG Kur 4 V g 1, Schreiben an v. Justi vom 19. Juni 1756.

177 Johann Matthias Gesner (1691-1761), war bereits ab 1734 Professor der Poesie und der Beredsamkeit in Göttingen. 
Orientalisten Johann David Michaelis, gegen den Ankauf der Sammlung aus. ${ }^{178}$ Gesner teilte zwar die Ansicht, dass es von Vorteil für die Universität wäre, eine Naturalien- und Kunstkammer in ihrem Umkreis zu haben, schränkte aber ein, dass es sich hierbei nicht um eine „public-anstalt“ handeln müsse. Es genüge, „wenn auch ein einiger [sic] Vielmehr dergleichen hat“. In Göttingen befanden sich zu dieser Zeit gleich zwei in Frage kommende Privatsammlungen, ${ }^{179}$ und so schrieb Gesner, man könne sich durchaus „mit der Gretzelischen u. Büttnerischen“ Sammlung begnügen, „sonderlich da die Büttnerische auf alle drey Natur-Reiche gehet“, einen beträchtlichen Umfang aufweise und auch über eine ebenso gut bestückte zugehörige Bibliothek verfüge. Daneben, beklagte Gesner, sei das Verzeichnis der angebotenen Sammlung „nicht hinlänglich, [um] zu urtheilen ob die Sammlung von der es handelt, von besondern Nutzen seÿn Könnte: noch wenig einen Überschlag des Preises zu machen“. Allerdings sei demselben wohl zu entnehmen, dass das angebotene Kabinett v.a. aus Petrefacten ${ }^{180}$ bestehe, ein Umstand, der gleich aus drei Gründen gegen die Anschaffung des Kabinetts spräche: zum einen seien diese „im Grätzelschen Cabinet ungemein vollständig“, zum anderen sei die Göttinger Umgebung so reich an Petrefakten, dass es widersinnig sei, viel Geld auszugeben um von weit her dergleichen Dinge kommen zu lassen, und drittens schreibt er: „,... petrefacta, [...], halte ich in Naturalien-Cabinetten für die Nebensache. Sie füllen uns ein sehr klein Capitel der historie naturalis.“ Er räumt anderen Bereichen weit größere Relevanz ein: „Was ein Cabinet auf Universitäten erst sehr nützlich macht, ist der Reichthum an Mineralien, Stufen, [...] und Erdfarben, Edelgesteinen, und Thieren, nicht aber [von] Petrefactis.“ Als letzten wichtigen Grund gegen die Anschaffung eines universitätseigenen Kabinetts führt Gesner räumliche und ökonomische Engpässe an. In der Bibliothek sei kein Platz für ein solches Kabinett und „Vor ein ander Zimmer, u. einem besondern Aufseher $\mathrm{Zu}$ fragen ist vermutl. aus Oeconomischen Umständen nicht thunlich.“ Auch in der Societät der Wissenschaften, die einen adäquaten Rahmen für ein Kabinett böte, und wo sich eventuell noch Platz für eine solche Sammlung finden ließe, würde selbige nur zur Zierde dienen, weil die Mittel zur Betreuung einer Sammlung

\footnotetext{
178 Die nachstehenden Zitate stammen sämtlich aus UAG Kur 4 V g 1.

179 PLISCHKE, Ethnographische Sammlung, 11, kommentierte 1931, dass sich diese beiden Sammlungen „vortrefflich ergänzten“.

180 Unter Petrefakten versteht man versteinerte Tiere und Pflanzen.
} 
fehlten. Folglich sei man mit den beiden umfangreichen Göttinger Privatsammlungen des Tuchfabrikanten Grätzel und des Naturforschers Büttner zunächst weit besser bedient. Gesner zeigte eine Alternative zum aktuellen Kaufangebot auf. Er schlug vor, die in Hannover von geringem Nutzen seiende ehemalige Schlütersche Sammlung nach Göttingen zu überführen, sobald sich eine Möglichkeit zur Unterbringung und Betreuung finde, und zunächst den bis dahin vergleichsweise gering vergüteten ${ }^{181}$ Büttner enger an die Universität zu binden, damit dessen wertvolle Sammlung nicht für den Standort Göttingen verloren gehe. Außerdem gab er der Überzeugung Ausdruck, dass es wohl möglich sei, die Grätzelsche Sammlung nach dem Ableben des Besitzers günstig für die Universität zu erwerben, solange man nicht auf bestimmte Stücke beharre. ${ }^{182}$ Ob es eine zeitnahe Reaktion auf Gesners Vorschläge gab und wie sie ausfiel, ist aus den Akten nicht nachzuvollziehen - jedenfalls kam es 1759 zu keinem Sammlungsankauf und mit dem Tod Gesners 1761 verlor das Projekt seinen Fürsprecher. Neuerliche Belege, die von der Absicht eines Sammlungserwerbs künden, finden sich erst etwa 14 Jahre später. Damit ist die eigentliche Entstehung des Academischen Museums Göttingen auf das Jahr 1773 zu datieren. Es handelt sich dabei jedoch nicht um eine Gründung im engeren Sinne, sondern um den Ankauf der bestehenden, bereits erwähnten Büttnerschen Sammlung. ${ }^{183}$

So heißt es in den Göttingischen Anzeigen von gelehrten Sachen vom 1. November 1773, dass im Rahmen der Feierlichkeiten zum Stiftungsfest der Universität ${ }^{184}$ bekannt gegeben worden sei, „daß die ansehnlichen Naturalien- und Münzsammlungen unsers Hrn. Prof. Büttners auf seinem [sic] eigenen patriotischen Betrieb an die Universitätsbibliothek überlassen sind“ und diese „so bald sie einige Einrichtung werden erhalten haben, zu dem öffentlichen Gebrauche auf gleiche Weise als die Bibliothek selbst sollen bestimmt werden.“185 Offensichtlich hatte man sich an das Anliegen

${ }^{181}$ Vgl. UAG Kur 4 V g 1. Dort wird Büttners Salaire mit 200 Rthl angegeben.

182 Alle Angaben aus: UAG Kur 4 V g 1, 12r u. v (Schreiben ohne Datierung).

183 Die Universität kaufte nur Büttners Naturalien- und Münzkabinett an. Seine umfangreiche Bibliothek blieb bis 1783 in seinem Besitz und ging dann an Herzog Carl August von Weimar über. Vgl. EHLERS, Göttinger Zoologen, 7.

184 Das Stiftungsfest fällt auf den 17. September.

185 Vgl. GGA vom 1. November 1773; Blumenbach bestätigte dies rückblickend mit den Worten: „Die Grundlage zum naturhistorischen Theil des Musei gab die Naturalien-Sammlung des vormaligen Professors Büttner, die er (1773) gegen Leibrente der Universität überließ.“ AVkG, Akten 1Altakten lose, Alte Dokumente 19. Jahrhundert, J.F. Blumenbach über die „Naturaliensammlung des academischen Musei“, 28. Oktober 1808. 
Gesners, die Büttnersche Sammlung für den Standort Göttingen zu bewahren, erinnert. Noch bevor man den Ankauf publik machte, musste man sich von dem Gedanken verabschieden, die Grätzelsche Sammlung hinzuzuerwerben. Die Antwort auf die am 5. Oktober 1773 an die Universitäts-Expedition ergangene Meldung, „daß der Manufacturier Graetzel daselbst sein Naturalien-Cabinet, so von dem Professor Johann Bekmann auf 5. bis 600 rthlr: geschätzet worden, zu verkaufen gewillet seÿ,“186 fiel negativ aus. Es hieß wiederum: „,[D]ie Universitätskaße, [sei] bei den, besonders auf Acquisitionen dieser Art, Zeither gehabten starken Ausgaben, nicht im Stande, desfals vor der hand um weiteres anzuwenden.“ ${ }^{187}$

Wie, wann genau und in welchem Zustand die Büttnersche Sammlung an die Universität gelangte, ist heute nicht mehr exakt zu ermitteln. Die Abnahme des Büttnerschen Kabinetts erfolgte offenbar im Sommer 1773 durch Heyne, dem „bei diesen Arbeiten und der nun notwendig gewordenen Verwaltung der Kandidat der Medizin Johann Friedrich Blumenbach half.““188 Dieser war im Wintersemester 1772/73 nach Göttingen gekommen und nahm bereits in diesem Semester am NaturgeschichteCollegium Büttners teil. Seine angeblich autobiographische, bei Marx abgedruckte Skizze $^{189}$ wirft ein etwas facettenreicheres Licht auf die Ereignisse, weiß er doch über den Ankauf der Sammlung zu berichten:

${ }^{186}$ UAG Kur 4 V g 3, Schreiben vom 5. Oktober 1773. Wann genau Grätzel das Angebot unterbreitete, ist nicht bekannt. Fest steht lediglich, dass der „Commercien-Obercommissarius Johann Heinrich Grätzel“ bereits am 25. März 1770 verschieden war; wahrscheinlich nahm die Erbengemeinschaft anschließend seine Verhandlungsposition ein. Vgl. UAG Kur 4 V g 3, Schreiben vom 19. Oktober 1773.

187 UAG Kur 4 V g 3, Schreiben vom 19. Oktober 1773. Der weitere Verbleib der Grätzelschen Sammlung ist nicht geklärt.

188 PlisCHKE, Ethnographische Sammlung, 12. Plischke beruft sich hier auf die Personalakte Büttners.

${ }^{189}$ In MARX, Andenken an Johann Friedrich Blumenbach heißt es auf Seite 4 ,Doch über die frühesten Anregungen und Förderungen, welche ihm teils im elterlichen Hause, theils bei seinem ersten Eintritte in die größere Welt zu Theil wurden, mag eine handschriftliche Mittheilung, welche ich der Güte des Verewigten verdanke, hier ihre Stelle finden, und somit deutlicher sprechen als jede historische Auseinandersetzung es vermag.“ Marx weist diese Notiz als Zitat aus, macht aber über die Herkunft des Schriftstückes keine genaueren Angaben. So ist z. B. der Zeitpunkt der Abfassung des Schriftstückes, sofern es tatsächlich existierte und aus Blumenbachs Hand stammte, unbekannt; die Art und Weise, in der es abgefasst ist, lässt lediglich darauf schließen, dass es retrospektiv niedergeschrieben wurde. In der zugehörigen Fußnote erläutert Marx: „Bl. hat ausser dieser obigen längern Mittheilung blos wenige flüchtige Notizen über sein Leben zurückgelassen. Diese habe ich, soweit sie zu meiner Kenntnis gelangten, sorgsam benutzt. Auf seine Absicht, eine Selbst-Biographie zu verfassen, scheinen zwei von ihm aufgezeichnete Stellen hinzudeuten. Plerique suam ipsi vitam narrare, fiduciam potius morum, quam arrogantiam rati sunt. - Sine gratia aut ambitione, bonae tantum conscientiae pretio ductus." Bei diesen Worten handelt es sich jedoch um ein - wenn auch relativ freies - Zitat aus Tacitus’ Agricola und nicht um eine Eigenkreation Blumenbachs. 
Gleich in jenem ersten Winter kam durch Heyne’s Vermittlung der Ankauf von Büttners Naturalien- und Münz-Cabinett für die Universität zu Stande. Nur war in der exemplarischen Unordnung, worin die Naturalien bei dem ohnehin nichts weniger als expediten Manne ganz unverzeichnet durcheinander lagen, ihm ein Gehülfe zum Ordnen und Abliefern nöthig. Heyne fragte ihn also: „Nun Sie lesen ja eben Naturgeschichte; haben Sie denn da unter Ihren jungen Leuten nicht etwa einen, der dazu passt?“ „Doch ja“, sagte Büttner, und nannte mich. „I, den kenn’ ich auch“ und so ward mir diese Hülfeleistung angetragen, die ich lehrreich fand und mit Vergnügen unentgeltlich übernahm. ${ }^{190}$

Dieser Bericht zeigt, dass der Erwerb der Sammlung mitnichten überraschend erfolgte, sondern dass der Bekanntgabe des Erwerbs ein halbes Jahr der Planung vorausgegangen war. Von weitaus größerer Bedeutung ist jedoch die Information, dass Büttners Sammlung offenbar weder - oder zumindest nicht den Ansprüchen an eine universitäre Sammlung entsprechend - geordnet noch beschriftet oder gar katalogisiert war. Von Interesse ist ebenfalls, dass Blumenbach, der erst 1776 offiziell zum Unteraufseher des Universitätskabinetts bestellt werden sollte, die spätere Museumssammlung quasi von seinen ersten Tagen in Göttingen an kannte und dass sein Einfluss auf das Museum bereits vor der Bekanntgabe des Erwerbs der Sammlung begann.

\subsection{2 „Das vorzügliche darin...“ - Wert und Umfang der Sammlung}

Welchen Inhalt, Umfang und Wert, welche Bedeutung die Sammlung Büttners besaß, lässt sich heute nur schwer ermessen. Die Universität erwarb Büttners Kabinett nicht gegen einen Festpreis, sondern gegen die Gewährung einer Leibrente. ${ }^{191}$ Laut Gesners Schreiben aus dem Jahr 1759 hatten Büttner und sein Vater vor ihm im Lauf der Zeit für das Kabinett und die zugehörige Bibliothek etwa 8000 Taler aufgewendet. ${ }^{192}$ Plischke betont den hohen Stellenwert der Sammlung, indem er eine Episode aus dem Siebenjährigen Krieg (1756-1763) ${ }^{193}$ heranzieht. So habe „eine französische Besatzung, die [...] in Göttingen lag, vor allem wegen dieser wertvollen Sammlung,

190 MARX, Andenken an Johann Friedrich Blumenbach, 6.

191 Vgl. UAG Kur 4 V g 19, 48r. Aufschluss über die Höhe der Leibrente könnte die Personalakte Büttners geben.

192 Eine andere, auf das Jahr 1761 zu datierende Wertangabe findet sich bei EHLERS, Göttinger Zoologen, 6. Dort wird der Gesamtwert der Bibliothek und der Sammlung auf 3000 Louis d'Or bemessen; Ebd., 4 gibt Ehlers zudem an, dass die Anfänge zu Büttners Sammlung schon durch dessen Großvater gelegt worden seien.

193 In Göttingen waren zwischen 1757 und 1762 verschiedene französische, zeitweilig auch preußische Besatzungstruppen stationiert. 
für die sich viele französische Offiziere interessiert hätten, sich außerordentlich schonend aufgeführt. “194

Doch damit ist kaum etwas über Inhalt und Umfang der Sammlung ausgesagt. Blumenbachs oben wiedergegebene Erinnerungen sowie die aktenmäßig belegbare Tatsache, dass er mit dem Ordnen der Sammlung und des dazugehörigen Kataloges beauftragt wurde, ${ }^{195}$ machen es unwahrscheinlich, dass vorab ein umfassender Katalog der Sammlung existierte, wie es im 18. Jahrhundert immer häufiger bei bürgerlichen Privatsammlungen vorkam. ${ }^{196}$ Wie bereits erwähnt, erstreckte sich die Büttnersche Sammlung „,auf alle drey Natur-Reiche“, ${ }^{197}$ was Pütter 1788 - sich auf die Anfänge des Museums beziehend, ohne jedoch einen näheren Zeitpunkt anzugeben - folgendermaßen ergänzte: „[d]ie Sammlungen bestanden überhaupt in Mineralien, Hölzern und Pflanzen, Thieren, edlen Steinen, ausländischen Kunstsachen und Münzen.“198 1808 präzisierte Blumenbach das im Rahmen seines kurzen Berichts über die Geschichte der „Naturaliensammlung des academischen Musei“ noch einmal:

Das vorzügliche darin waren die Thiere in Spiritus; und unter den Mineralien außer einer Anzahl roher, geschliffner und geschnittner Edelsteine, nur einzelne andre Foßilien von wißenschaftlichen Werth; beÿ weitem die mehresten übrigens in zu kleinen oder verstoßnen Stücken, oder zu unbedeutend gemein. Gar manches auch sehr irrig angegeben. ${ }^{199}$

Damit wäre eine grobe qualitative Eingrenzung geschaffen. Bleibt die Frage nach dem Umfang der Bestände des Academischen Museums. Die Größe des Grundbestandes ist zwar nicht bekannt, doch kann man ihn anhand der erhaltenen Quellen in etwa abschätzen. 1776 wurde Blumenbach damit beauftragt, einen Katalog des Museums zu erstellen, dessen Fertigstellung Heyne im Oktober 1778 an die „Königlich Großbritannischen zur Churfürstl. Braunschweig-Lüneburgischen Landesregierung höchstverordneten Herren geheime Räthe“ meldete. ${ }^{200}$ Bis einschließlich 1778 lassen

194 PLisChKE, Ethnographische Sammlung, 12. Vermutlich bezieht Plischke sich hier wiederum auf die schon mehrfach zitierte Kuratorialakte 4 V g 1 des Göttinger Universitätsarchivs. Leider ist gerade diese Stelle der Akte nur schlecht zu entziffern. Sicher geht aber daraus hervor, dass Büttner seit der Zeit der französischen Besatzung mit einem Chevalier de Grottier korrespondierte.

195 Vgl. UAG Kur 4 V g 5.

196 Zur Funktion der Kataloge vgl. ausführlich das gleichnamige Kapitel bei VALTER, Kunst- und Naturaliensammlungen, 39-47.

197 UAG, Kur 4 V g 1.

198 JOHANN STEPHAN PÜTTER, Johann Stephan Pütters Versuch einer academischen GelehrtenGeschichte von der Georg-Augustus-Universität zu Göttingen. Theil 2: Von 1765-1788, Göttingen 1788, 232.

199 AVkG, Akten 1-Altakten lose, Alte Dokumente 19. Jahrhundert, J.F. Blumenbach über die „Naturaliensammlung des academischen Musei“, 28. Oktober 1808.

${ }^{200}$ Vgl. UAG Kur 4 V g 11. 
sich in den Verwaltungsakten nur zwei Sammlungszugewinne belegen: zum einen „[d]ie von Sr. Kgl. Majestät dem dortigen Museum geschenkten, bei hiesiger Bibliothek bisher aufbewahrten Erzstufen und Drusen, auch großen Silberstufen““201, deren Umfang sich leider nicht nachweisen lässt, und zum anderen eine Schenkung von Naturalien der verwitweten Fürstin von Waldeck im Umfang von 162 Nummern. ${ }^{202}$ Sollte es sich dabei tatsächlich um die einzigen Zugewinne nach dem Ankauf der Sammlung handeln, so ließe sich der Grundbestand mit gut 12000 Objekte angeben. $^{203}$

\subsubsection{Institutionelle Zuordnung und personelle Ausstattung}

Soweit sich dies heute noch nachverfolgen lässt, machte das Museum in den ersten Jahren seiner Existenz im öffentlichen Raum wenig von sich reden: Über die ersten Jahre des Museums geben fast ausschließlich Verwaltungsquellen Auskunft. Zieht man die personelle Situation des Museums und die Tatsache in Betracht, dass die nicht gerade kleine Sammlung Büttners ${ }^{204}$ komplett neu geordnet und gegebenenfalls be- und verzeichnet werden musste, bevor sie zugänglich gemacht wurde, scheint dies nicht weiter verwunderlich.

Personell und institutionell war das Museum Heyne unterstellt worden. 1763 als Professor eloquentiae et poesis und Bibliothekar an die Georgia Augusta berufen, wirkte er hier letztlich „fast ein halbes Jahrhundert als Philologe, als Direktor der Universitätsbibliothek, als Schulreformer, als Organisator in allen Bereichen“. ${ }^{205}$ Über die Jahre erhielt er einen solchen Machtzuwachs, dass ihm - gemeinsam mit dem Hannoverschen Kanzleisekretär und Expedienten in Universitätssachen Georg Friedrich

201 Vgl. UAG Kur 4 V g 6.

202 Vgl. UAG Kur 4 V g 4 . Hier sind die Nummern einzeln aufgeschlüsselt.

203 Bei der weitaus überwiegenden Zahl der Objekte handelt es sich um Mineralien. Da hier zu wenig Raum ist, um en detail auf die Aufschlüsselungen der Objekte im Catalogus Musei Academici einzugehen, werden im Anhang die einzelnen Kategorien und die Anzahl der ihnen zugeordneten Objekte aufgeführt.

204 Im Aktenbündel UAG Kur 4 V g 1 findet sich die Notiz: „Die [sic] Cabinet nimmt die ganze mittlere Etage des Hauses ein, welches der sel. Canzler von Mosheim bewohnet hat.“ PLISCHKE lokalisiert dieses Haus als das „heutige Prinzenhaus, Prinzenstraße 2“. Vgl. Ders., Ethnographische Sammlung, 12.

205 CHRIstof BOEHRINGER, Über die Göttinger Sammlung von Gipsabgüssen antiker Skulpturen, in: Hoffmann/Maack-Rheinländer (Hg.), „Ganz für das Studium angelegt“, 64-72, 64. 
Brandes - heute die faktische Leitung der Universität zugeschrieben wird. ${ }^{206}$ Die Arbeit im Museum war also bei weitem nicht die einzige Tätigkeit Heynes, und die Quellen erwähnen keinen weiteren Angestellten, sodass davon auszugehen ist, dass sich das Personal des Museums zu dieser Zeit wohl auf Heyne selbst, die studentische Hilfskraft Blumenbach und eventuell noch den Nachtwächter der Bibliothek beschränkte. Sollte das Museum also irgendwann einen Aufschwung erfahren, so musste zunächst Personal angestellt werden, um die Sammlungen publikumstauglich zu machen. Folgerichtig fiel hier die Wahl auf Blumenbach. In seinen Lebenserinnerungen führt Blumenbach aus:

Einige Zeit nachdem schon allerhand abgeliefert und einstweilen ins vormalige medicinische Auditorium gebracht war, kam der würdige Minister und Curator der Universität, von Lenthe, hieher, besah unsere Institute, und da sollten ihm doch auch diese Sachen gewiesen werden, und weil der ehrliche Büttner dazu nicht eben geeignet schien, so ward ich eilig gerufen, und machte meine Sache so leidlich, dass der Minister gleich beim Herausgehn Heynen bei Seite genommen und gesagt hat: ,Den jungen Mann müssen wir hier behalten.' - Nachdem ich im Herbst 75 am Anniversarium der Universität promovirt hatte, hielt ich gleich im nächsten Winter als Privatdocent meine ersten Vorlesungen über die Naturgeschichte und ward noch in diesem Semester im Febr. 76 zum ausserordentlichen und hernach im Nov. 78 zum ordentlichen Prof. der Medicin ernannt. $^{207}$

Die Verwaltungsakten aus dem Bestand des Academischen Museums stellen den Sachverhalt etwas nüchterner dar. ${ }^{208}$ Erste Erwägungen, Blumenbach als Unteraufseher des Museums anzustellen, lassen sich bereits für Juli 1775 in den Akten belegen. Dass dieser die erste Wahl war, geht daraus ebenso eindeutig hervor wie die Tatsache, dass es einige Überlegung gekostet haben mag, ihm zugleich auch eine Stelle anzuweisen, mit der er seinen Lebensunterhalt verdienen konnte - ohne dass damit andere vor allem ältere Kollegen übergangen oder vor den Kopf gestoßen würden. Das ganze Unternehmen bedurfte also der sorgfältigen Vorbereitung. Am 24. Februar 1776 wurde Johann Friedrich Blumenbach formell zum außerordentlichen Professor an der Universität Göttingen ernannt. Zugleich wurde ihm „die Unteraufsicht über das dortige Kunst- und Naturalien-Kabinett nebst der Besorgung des

206 Vgl. WOLfGANG BÖKER, Christian Gottlob Heyne und das Haus Papendiek 16 - ein Professor wird Göttinger, in: Göttinger Jahrbuch 50 (2002), 93-111, 103; CARL HAASE, Göttingen und Hannover. Geistige und genealogische Beziehungen im ausgehenden 18. Jahrhundert, in: Göttinger Jahrbuch 15 (1967), 95-124, 111.

207 MARX, Andenken an Johann Friedrich Blumenbach, 6.

208 Über die Ernennung Blumenbachs als Unteraufseher des Museums gibt das Aktenbündel UAG Kur 4 V g 5 ausführlich Auskunft. 
Prosektorats $^{209}$ bei der Anatomie“ ${ }^{210}$ übertragen und seine Besoldung auf 100 Rthlr $^{211}$ jährlich festgelegt. Parallel zu dieser Ernennung erging eine ausführliche Dienstanweisung an den frischgebackenen Professor extraordinario Blumenbach. Dieser kommt aufgrund der geringen Anzahl an Quellen zur museologischen Praxis in Göttingen eine außerordentliche Bedeutung zu, weshalb sie hier in Gänze wiedergegeben werden soll.

1. Ausser der allgemeinen Verbindlichkeit und Verpflichtung zur Treue und Gewissenhaftigkeit bey den ihm untergebenen Naturalien und andern Sachen von Werth, deren gute Erhaltung, Ordnung, vortheilhaffte Aufstellung und sichre Verwahrung, so wie die Reinlichkeit und äußerliche gute Aufsicht des Kabinets, er sich überhaupt nach beßtem Wissen und Vermögen angelegen seyn lassen wird, macht er sich ist derselbe insonderheit anheischig verpflichtet, und dahin angewiesen,

2. daß, so wie nach und nach die Schränke und Vorrichtungen im Kabinet gefertiget seyn werden, er die Naturalien mit Zubehör hineinschaffen und aufstellen wird, auf ihre Classen, und in der beßten Ordnung.

3. Ein gleiches wird er in Ansehung der vom Prof. Büttner etwa noch nachgelieferten oder gekauften und sonst an das Kabinet gekommenen Stücke und zwar ohne unnöthigen Aufschub und mit dem möglichsten Betrieb, ohne Zeitverlust beobachten.

4. Eben so gewissenhaft wird er sich die Verfertigung, Beendigung und Berichtigung der Catalogen des Kabinets angelegen seyn lassen; von welchen, so wie von den Nachträgen, von Zeit zu Zeit, ein Exemplar, das vom Hofrath Heyne untersignirt ist, an Königl. Regierung eingesendet werden muß.

5. Ihm lieget das Herumführen der Fremden und der hiesigen Liebhaber vorzüglich ob, und so, wie sich der Hofrath Heyne, so ofte sich Fremde an ihn wenden, ihn, den Unteraufseher ersuchen wird, zugegen zu seyn, so wird er hinwiederum den Hofrath Heyne von der Gegenwart derer benachrichtigen, die sich bey ihm melden werden, um herumgeführet zu seyn. Insonderheit gilt dieß bey den hier studierenden, die das Kabineet besehen wollen, tnd $z \nVdash-d e n e n$ willfährig zu seyn, sich ohnedem leicht eine Zeit voraus verbreden läßt. Er wird auch hirbey alle Vorsicht brauchen, daß keine zu grose Anzahl, auch nicht allerhand Personen ohne Auswahl, in das Kabinet eingelassen werden; zumal so lang die Sachen noch nicht unter den gehörigen Beschluß gebracht sind.

6. So wie ihm der gewissenhafte Gebrauch des Kabinets und der einzelnen Stücken zu seinem Studieren und Vorlesungen gegönnet wird: So versteht es sich auf der andern Seite, daß er alle Beschädigung verhüte, und daß er nichts aus dem Kabinet vertauschen, veräusern, oder an andere verabfolgen lassen kan. Eben so wenig Kan er an hiesige Liebhaber, Professoren und Studirende, etwas verleihen, ohne vorher mit dem Hofrath Heyne darüber Abrede zu nehmen, welcher, nach Erforderung der Umstände an Königl. Regierung gehörige Anzeige davon thun wird. Ueber das Ausgeliehene aber wird eine ordentlich-abgefaßte Registratur gehalten.

${ }^{209}$ Nach Meyers Konversationslexikon. Ein Nachschlagewerk des allgemeinen Wissens. Fünfte, gänzlich neubearbeitete Auflage. Vierzehnter Band. Politik bis Russisches Reich, Leipzig und Wien 1896, ist ein Prosektor „(lat., ,Vorschneider, Zergliederer'), [ein] in anatomischen Lehranstalten der dem Lehrer beigegebene Gehilfe, welcher die Leichen zur Demonstration vorzubereiten und die anatomischen Präparate für den Unterricht oder das anatomische Museum der Anstalt anzufertigen hat. In größern Krankenanstalten ist P. der pathologische Anatom, welcher mit der Untersuchung der Leichen zur Prüfung der Diagnose und der angewandten Heilmittel betraut ist.“ Der Eintrag zeigt anschaulich, wie etabliert die Institution Museum an der Wende zum 20. Jahrhundert geworden war. Im Zedler findet sich dagegen noch kein Eintrag zum Begriff Prosektor.

${ }^{210}$ UAG Kur 4 V g 5, 11r.

${ }^{211}$ An anderer Stelle wird in derselben Quelle darauf hingewiesen, dass er „mit Einschluß der LicentAcquivalentzgelder 140 rthlr fürs erste zu genießen hätte“. Vgl UAG Kur 4 V g 5, 4v. 
7. So oft der Hofrath Heyne verhindert wird, die Münzen und die edlen Steine, Fremden und Personen, denen man es nicht wohl abschlagen kan, vorzuzeigen, so wird er, der Unteraufseher, den Schlüssel zum Schranke von ihm erhalten, und bey der Vorzeigung alle erforderliche Vorsicht brauchen, auch so gleich den Schrank sorgfältig wieder verschliessen, und die Schlüssel wieder an besagten Hofrath Heyne zurückgeben.

8. Gleichwie er sich bisher durch sein Verhalten gegen den Professor Büttner vielen Beyfall erworben hat, so sucht er auch forthin demselben alle mögliche Gefälligkeit, Nachgeben und Gelehrigkeit zu beweisen.

9. Bey entstehender Feuersgefahr oder andern Umständen dieser Art, siehet er das Kabinet als den Posten an, auf dem er sich unverzüglich einzustellen und alles Mögliche zu Erhaltung und Rettung des Kabinets beyzutragen hat.

10. Er bestrebet und beeifert sich dahin, das Kabinet so weit in Ordnung zu bringen, zu Ausgang des Sommers 1776 alles nach dem Catalogo revidirt werden kan. ${ }^{212}$

Neben Anhaltung zu allgemeiner Sorgfalt, Verbindlichkeit und Treue des Unteraufsehers Blumenbach werden hier hierarchische Strukturen und Kompetenzaufteilungen herausgestellt. So wurde Blumenbach nicht nur ausdrücklich Heyne untergeordnet, sondern ihm wurde auch die Aufsicht über die Objekte mit geringerem Materialwert übertragen. ${ }^{213}$ Der eigentliche Wert der Quelle liegt aber in den Ausführungen zur museologischen Praxis im Academischen Museum, die sich in dieser Form nirgendwo sonst finden. Rechte und Pflichten des neuen Angestellten und sein Umgang mit verschiedenen Personenkreisen wurden genau festgelegt. Nachgewiesen wird hiermit auch die Bestimmung des Museums für den öffentlichen Gebrauch - und zwar schon, bevor alle Objekte die ihnen zugedachten Orte eingenommen haben. „Fremde“, „hiesige Liebhaber“ und „hier studierende“ sollten nach vorheriger Anmeldung umhergeführt werden. Für die Ausstellungsstücke sollten nach und nach spezielle Schränke gefertigt werden, und es wurde eine „vorteilhaffte Aufstellung und sichre Verwahrung“ derselben eingefordert. Doch auch Ausschlusskriterien für das Publikum wurden festgelegt: Es sollen keine zu großen Gruppen und „auch nicht

${ }^{212}$ UAG Kur 4 V g 5, 12r-15r.

213 Die nächst höhere Instanz nach Heyne, mit der Museumsangelegenheiten verhandelt wurden, war für die Zeit bis zur Eingliederung Göttingens in das Königreich Westphalen das Geheime Ratskollegium in Hannover. Innerhalb des Untersuchungszeitraumes 1773-1840 veränderte sich mehrfach die Verwaltungsstruktur der Hannoverschen Regierung respektive Zuständigkeitsbereiche oder Bezeichnungen. Einen Sonderfall stellt die Verwaltungsstruktur in der Zeit der napoleonischen Ära (1806-1813) dar. Einen hilfreichen Überblick verschafft hier die mehrbändige Deutsche Verwaltungsgeschichte, insbesondere die Artikel: THOMAS KLEIN, Königreich Hannover, in: Kurt G. A. Jeserich/Hans Pohl/Georg-Christoph von Unruh (Hg.), Deutsche Verwaltungsgeschichte. Bd II: Vom Reichsdeputationshauptschluß bis zur Auflösung des Deutschen Bundes, Stuttgart 1983, 678715; FRANZ-LUDWIG KNEMEYER, Rheinbundstaaten 2. Die Verwaltungsorganisation des Königreichs Westphalen, im selben Band, 336-339; GÜNTER SCHEEL, Kurbraunschweig und die übrigen welfischen Lande, in: Kurt G. A. Jeserich/Hans Pohl/Georg-Christoph von Unruh (Hg.), Deutsche Verwaltungsgeschichte. Bd I: Vom Spätmittelalter bis zum Ende des Reiches, Stuttgart 1983, 741763, und DieTMAR WiLloweIT, Die Universitäten, im selben Band, 369-383. 
allerhand Personen ohne Auswahl in das Kabinett eingelassen werden“. Wesentliches Argument hierfür ist der Schutz der Bestände. Interessanterweise wurden jedoch bestimmte Gruppen wie z.B. Kinder oder Angehörige bestimmter gesellschaftlicher Schichten oder Berufsgruppen nicht namentlich ausgeschlossen. Dennoch dürfte die Notwendigkeit einer vorherigen persönlichen Anmeldung und ein - für diese Zeit allerdings noch nicht belegbares - Eintrittsgeld eine gewisse Schwellenfunktion für einen Museumsbesuch ausgeübt haben.

In den Aufgabenbereich des Unteraufsehers, der wohl nicht für alle in der Dienstanweisung angeführten Punkte persönlich zuständig, aber doch für ihre Ausführung und Befolgung verantwortlich gewesen sein wird, fielen darüber hinaus die „Reinlichkeit und äußerliche gute Aufsicht des Kabinets“ sowie die Sorgfaltspflicht für das Anordnen der Objekte. Wir erfahren, dass den Ausstellungsstücken die „beste Ordnung“ gegeben werden sollte, die Erstellung dieser Ordnung aber nicht - in schriftlicher Form - festgelegt wurde, sondern anscheinend in weiten Teilen Blumenbach überlassen blieb. Ferner sollte ein stets zu aktualisierender Katalog verfertigt und die Regierung in Hannover „von Zeit zu Zeit“ über Sammlungszugewinne informiert werden. Fraglich bleibt, ob das Interesse der Geheimen Räte über die Ansprüche einer geordneten Buchführung hinausging.

Im Gegensatz zu Fremden, Liebhabern und Studierenden (übrigens eine beachtenswerte Gegensatzkonstruktion) genoss Blumenbach - und wohl auch Heyne in seiner Funktion als (Ober-) Aufseher des Academischen Museums - uneingeschränkten Zutritt zum Kabinett und zu den einzelnen Stücken, deren gewissenhafter Gebrauch ihm zu „seinem Studieren und Vorlesungen gegönnet wird“. Dem Unteraufseher kam folglich nicht nur die Funktion zu, die Sammlung zu ordnen und sie inhaltlich zu erschließen, sondern auch die Aufgabe, die einzelnen Stücke oder auch die gesamte Sammlung in wissenschaftliches Arbeiten einzubeziehen. Also nicht nur an den Beständen zu arbeiten, sondern auch mit ihnen. Dies bedingt eine gewisse Form der Publikmachung: Blumenbach wurde damit eine Multiplikatorrolle zugewiesen.

Ob es tatsächlich zur Ausleihe von Sammlungsstücken kam, wie in der Dienstanweisung berücksichtigt, ist nicht bekannt. Jedenfalls ist keine Registratur darüber erhalten.

Damit ist die personell-institutionelle Situation des Museums in seiner Gründungs- 
phase zunächst geklärt - doch wo spielte sich all dies ab? Anders als man dies von Museumsgründungen - vor allem für naturhistorische Museen - aus der Mitte des 19. Jahrhunderts und verschiedenen dazu erschienenen Publikationen kennt, ${ }^{214}$ entstand in Göttingen 1773 kein eigenes Gebäude, um die neuerworbene Universitätssammlung unterzubringen. In den Quellen werden keine architektonischen Umbaumaßnahmen der ehemaligen Bibliotheksräume erwähnt, die besondere Hinweise auf ein Museum liefern würden, auf dessen Bedürfnisse zugeschnitten wären oder gar in ihrer äußeren Form auf den Inhalt verwiesen. Dennoch: Das Academische Museum entstand 1773 am zentralen Ort des Göttinger Universitätslebens, im alten Kollegienhaus, in dem sich neben der Bibliothek verschiedene Auditorien befanden. ${ }^{215}$ Die genaue Lage des Museums erschließt sich u.a. durch die bei Marx wiedergegebenen Lebenserinnerungen Blumenbachs. Dort findet sich der Vermerk, dass die ehemalige Büttnersche Sammlung „einstweilen ins vormalige medicinische Auditorium“²16 gebracht worden sei, was eine Verortung im südöstlichen Trakt des Erdgeschosses des Kollegienhauses ermöglicht. ${ }^{217}$ In der nachstehenden Abbildung (Abb. 3) ist der Standort des Museums farblich hervorgehoben. Der Raum ist dort als „Auditorium Philosophicum“ bezeichnet, das dort untergebracht war, bevor der Raum als medizischer Hörsaal genutzt wurde. ${ }^{218}$

214 Hier seien exemplarisch genannt: SuSAN SHEETS-PyEnSON, Cathedrals of science. The development of colonial natural history museums during the late nineteenth century, Kingston u.a.1988; YANNI, Nature's Museums.

215 Grundrisse mit wechselnder Belegung der Räume finden sich bei WERNER SEIDEL, Baugeschichte der Niedersächsischen Staats- und Universitätsbibliothek in Göttingen 1734-1953, Göttingen 1953.

216 MARX, Andenken an Johann Friedrich Blumenbach, 6.

217 Vgl. auch PÜTTER, Gelehrten-Geschichte (2), 219; ferner: PLISCHKE, Ethnographische Sammlung, 33, der das Museum ,in einem unteren Saal“ der Universitätsbibliothek lokalisiert; sowie KARL Julius HARTMANN/HANS FÜCHSEL (Hg.), Geschichte der Göttinger Universitäts-Bibliothek. Mit 17 Lichtdrucktafeln, Göttingen 1937, 100, die angeben: „Die wichtigste Veränderung [in der Raumaufteilung des Kollegienhauses] war, daß der östliche Hörsaal im unteren Stockwerk für das Heynes Oberaufsicht unterstellte Naturalienkabinett umgebaut wurde. Heyne klagte wiederholt über Raummangel, erhielt auch einige Repositorien und Schränke bewilligt.“

218 Vgl. KuRT SchELLENBERG, Der Borheck-Bau und seine Vorläufer: Beitrag zur Grundrißgeschichte der Göttinger Universitäts-Bibliothek (1767-1787), in: Universitätsbibliothek Göttingen (Hg.), Beiträge zur Göttinger Bibliotheks- und Gelehrtengeschichte, Göttingen 1928, S. 55-74, 56. 


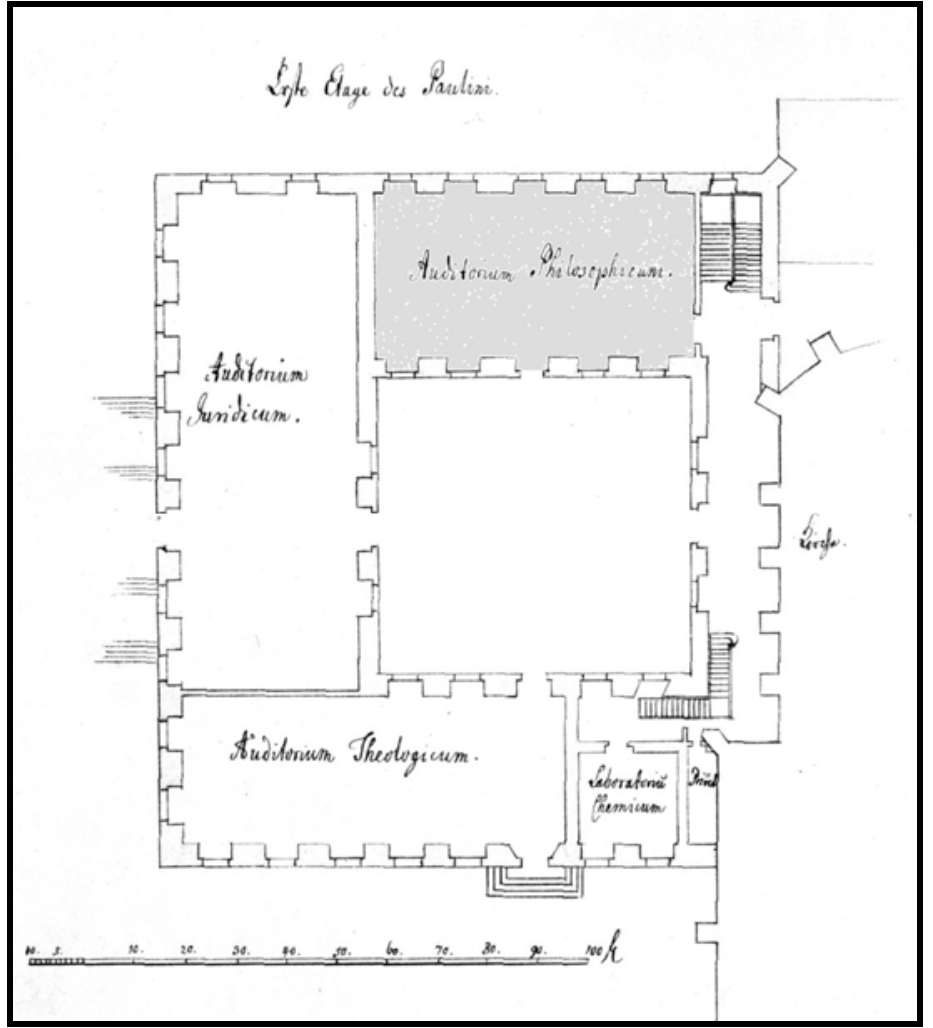

Abbildung 3: Erdgeschoss des Kollegienhauses (ca. 1734)

Über die „Ordnung der Dinge“ ${ }^{219}$ in der Anfangsphase des Museums gibt es keine zeitgenössischen Dokumente. Erst fünfzehn Jahre und etliche Sammlungszugewinne später berichtete der Universitätschronist Pütter von den verschiedenen Abteilungen des Museums und seinen Objekten. ${ }^{220}$

\subsubsection{Entstehungszusammenhang und universitäre Rahmenbedingungen}

Bis hierhin wurde gezeigt, über welche personelle und materielle Grundausstattung das Museum in seinen Anfängen verfügte und wie mit diesen Ressourcen umgegangen worden ist. Doch welche Rolle nahm das Museum im universitären Gefüge Göttingens ein? ${ }^{221}$ In welchem Zusammenhang standen andere Universitätssammlungen mit den Beständen des Academischen Museums?

219 FouCAUlt, Ordnung der Dinge, Titel.

220 Zur Aufteilung des Academischen Museums vgl. Kap. 3.3.

${ }^{221}$ Dieser Exkurs muss notwendigerweise kursorisch bleiben. Einen umfassenden Überblick (mit sehr ausführlicher Bibliographie) über v. a. geistige Entwicklungslinien der Göttinger Universität bietet LUIGI MARINO, Praeceptores Germaniae. Göttingen 1770-1820, Göttingen 1995; Darüber hinaus existiert eine fast unüberschaubare Anzahl an zeitgenössischen Dokumenten. Allen voran ist hier der von Pütter begonnene und von Saalfeld, später Oesterley fortgesetzte Versuch einer academischen Gelehrten-Geschichte von der Georg-Augustus-Universität zu Göttingen, 4 Teile, 1765- 
1734/37 222 als Reformuniversität gegründet und geistig v.a. der 1694 gegründeten Universität Halle verbunden, schwang sich Göttingen in den folgenden 50 Jahren zur „bedeutendste[n] Universität des 18. Jahrhunderts““223 auf, die eng dem Gedankengut der Aufklärung verpflichtet war. Im Jahr 1781 stand die Universität in voller Blüte: „Mit 947 Studenten erreicht die Universität im Sommer-Semester die Höchstzahl des 18. Jahrhunderts.“ ${ }^{224}$ Den Studierenden standen etwa 80 Lehrende ${ }^{225}$ an vier Fakultäten gegenüber. Nachdem dieser Höhepunkt „überschritten ist, sinkt die Frequenz [...] erneut bis zu den Freiheitskriegen (1813-1815) kontinuierlich ab.“ ${ }^{226}$ Zu Beginn des 19. Jahrhunderts verlor Göttingen stark an Attraktivität und an Zulauf. Geschwächt durch verschiedene Verwaltungsumstellungen im Zuge der napoleonischen Zeit (1806-1813) und sogar von der Schließung bedroht, erfreuten sich andere geistige Zentren wie beispielsweise die neu gegründete Berliner Universität (1810) weitaus größerer Beliebtheit. Eine weitere Krisensituation für die Universität ergab sich mit der Aufhebung des Staatsgrundgesetzes unter Ernst August von Hannover 1837 und der folgenden Protestaktion der ,Göttinger Sieben’. Obwohl sich im 19. Jahrhundert immer wieder bedeutende Wissenschaftler in Göttingen einfanden, kam es erst nach der Reichsgründung 1871 zu einer neuen Blütezeit.

Doch zurück ins 18. Jahrhundert. Noch befand sich die junge Universität, die vor allem Adelige anzog, im Aufschwung und es entstanden zahlreiche Einrichtungen, die wissenschaftlichen Bedürfnissen und wissenschaftlicher Reputation dienten und auch dem „ausgeprägte[n] Bedürfnis nach fürstlicher Selbstdarstellung““227 genüge

1838 zu nennen; Eine - ideologisch nicht ganz unbedenkliche - Gesamtdarstellung lieferte zuletzt GÖTZ VON SELLE, Die Georg-August-Universität zu Göttingen 1737-1937, Göttingen 1937; Diverse historische Darstellungen beleuchten Teilaspekte derUniversitätsgeschichte, so z.B. ULRICH HunGER, Die Georgia Augusta als hannoversche Landesuniversität. Von ihrer Gründung bis zum Ende des Königreichs, in: Ernst Böhme/Rudolf Vierhaus (Hg.), Göttingen. Geschichte einer Universitätsstadt, Bd 2: Vom Dreißigjährigen Krieg bis zum Anschluss an Preußen - Der Wiederaufstieg als Universitätsstadt (1648-1866), 139-213; und UlRICH HungER, Die Georg-AugustUniversität um 1800, in: Elmar Mittler (Hg.), „Der gute Kopf leuchtet überall hervor“: Goethe, Göttingen und die Wissenschaft, Göttingen 1999, 28-39.

${ }^{222}$ Der Vorlesungsbetrieb begann 1734, die feierliche Inauguration wurde jedoch erst am 17. September 1737 begangen.

${ }^{223}$ WILLOWEIT, Die Universitäten, 373.

${ }^{224}$ http://www.stadtarchiv.goettingen.de/chronik/1781.htm, Zugriff am 1. Januar 2005.

225 Pütter verzeichnete 1788 insgesamt 83 Lehrende, davon 34 ordentliche und 14 außerordentliche Professoren sowie 35 Dozenten. Vgl. PÜTTER, Gelehrten-Geschichte (2), Inhaltsverzeichnis.

226 DiETRICH VON MAERCKER, Die Zahlen der Studierenden an der Georg-August-Universität in Göttingen von 1734/37 bis 1978, in: Göttinger Jahrbuch 27 (1979), 141-158, 143.

227 WILLOWEIT, Die Universitäten, 373. 
leisteten. Dazu gehören der bereits ab 1736 eingerichtete und 1740 eröffnete Botanische Garten und die auf Betreiben Albrecht von Hallers 1751 ins Leben gerufene Akademie der Wissenschaften. Die für eine standesgemäße Ausbildung Adeliger obligatorische Reitbahn und der Fechtboden waren bereits 1734 bzw. 1742 entstanden.

Hier aber sind vor allem die zahlreichen Sammlungen von Interesse, die um die Mitte des 18. Jahrhunderts mehr oder weniger parallel zum Academischen Museum entstanden. Einen hervorragenden Überblick über diese Entwicklungen bietet der 2001 in Göttingen erschienene, von Dietrich Hoffmann und Kathrin Maack-Rheinländer herausgegebene Sammelband „Ganz für das Studium angelegt“. Die Museen, Sammlungen und Gärten der Universität Göttingen. ${ }^{228}$ Folgt man den Beiträgen der einzelnen Autoren, so gab Christian Gottlob Heyne den Anstoß zu zahlreichen Sammlungen. Auf sein Betreiben sind die heutige Originalsammlung des Archäologischen Instituts (um 1767), ${ }^{229}$ die Sammlung von Gipsabgüssen antiker Skulpturen (um 1766) ${ }^{230}$, die Göttinger Münzsammlung ${ }^{231}$ (deren Grundbestand aus dem Büttnerschen Ankauf stammt) und der Ankauf des Diplomatischen Apparats (1802) ${ }^{232}$ zurückzuführen. Darüber hinaus besaß die Universität(sbibliothek) seit ihrer Gründung eine wachsende Anzahl von Instrumenten ${ }^{233}$ und Modellen, ${ }^{234}$ für die zu einem nicht näher bekannten Zeitpunkt eine eigene Modellkammer eingerichtet wurde. Mit der Anlieferung der Uffenbach’schen Sammlung, ${ }^{235}$ die sich bis ins Jahr 1770 hinzog,

228 HOFFMANN/MAACK-RHEINLÄNDER (Hg.), „Ganz für das Studium angelegt“.

${ }^{229}$ Vgl. DANIEL GraePleR, Die Originalsammlung des Archäologischen Instituts, in: Hoffmann/Maack-Rheinländer (Hg.), „Ganz für das Studium angelegt“, 55-63.

230 Vgl. BOEHRINGER, Sammlung von Gipsabgüssen, in: Hoffmann/Maack-Rheinländer (Hg.), „Ganz für das Studium angelegt“", 64-72.

231 Vgl. Christof BOEHRINGER, Die Göttinger Universitäts-Münzsammlung, in: Hoffmann/MaackRheinländer (Hg.), „Ganz für das Studium angelegt“, 73-81.

232 Wolfgang PeTKE, Diplomatischer Apparat, in: Hoffmann/Maack-Rheinländer (Hg.), „Ganz für das Studium angelegt“", 82-90.

233 Bereits die zum Grundbestand der Universitätsbibliothek zählende sog. Bülowsche Sammlung beinhaltete eine „stattliche Reihe astronomischer, mathematischer und optischer Instrumente“. HARTMANN/FÜCHSEL, Universitäts-Bibliothek, 16.

234 Darunter fallen z. B. das Modell der „Hamelnschen Schleuse“, das „Modell der curieusen Londenschen Brücke, welche über die Themse daselbst erbauet worden“ und das Modell einer Papiermühle. Vgl. Georg W. Behre, Die Modellkammer der Universität Göttingen und deren Nutzung, in: Sächsische Landesstelle für Museumswesen (Hg.), Technische Modelle als Museumsbestand. Berichte und Erfahrungen, Chemnitz 1999, 4-13.

235 Bereits 1736 sagte der Frankfurter Architekt Johann Friedrich Armand von Uffenbach (16871769) der neu gegründeten Universitätsbibliothek die Schenkung seiner eigenen Bibliothek zu, die vor allem mathematische, physikalisch-technische, militärische, topografische und kunsthistori- 
gelangten neben weiteren Modellen und Instrumenten vor allem Kupferstiche und Handzeichnungen an die Göttinger Universität, für die eine Unterbringungsmöglichkeit geschaffen bzw. gefunden werden musste. Die genannten Sammlungen scheinen sich in ihrer Entstehungsphase stets in räumlicher Nähe zueinander, nämlich in oder zumindest nahe bei der Universitätsbibliothek befunden zu haben. ${ }^{236}$ Insofern erscheint die Bibliothek als logischer Standort für die Einrichtung des Academischen Museums. Ob dem Erwerb der einzelnen Sammlungen ein schlüssiges Gesamtkonzept zu Grunde lag bliebe zu untersuchen, ist aber nicht wahrscheinlich.

\subsubsection{Nomen est Omen?}

Ebenfalls ungeklärt ist, wann die Sammlung offiziell den Namen Academisches Museum bzw. Königlich Academisches Museum erhielt. Die früheste ungedruckte, diese Bezeichnung enthaltende Quelle ist beachtenswerterweise der Catalogus Musei Academici bzw. das Schreiben Heynes an die Regierung in Hannover, das dessen Fertigstellung im Herbst 1778 ankündigt. ${ }^{237}$ Vorher ist, auch in der Dienstanweisung Blumenbachs, nicht von einem Museum, sondern wahlweise von einem NaturalienKabinett, Universitäts-Kabinett oder academischem Kabinett die Rede. ${ }^{238}$ Die früheste gedruckte Quelle, die die Bezeichnung Academisches Museum verwendet, ist vermutlich Lichtenbergs Artikel im Göttinger Taschen Calender aus dem Jahr 1779. ${ }^{239}$ Hier kommt die bereits in Kapitel 2 angerissene Differenzierungsproblematik zwischen Naturalienkabinetten und Museen erneut zum Tragen. Betrachtet man die Frühzeit des Academischen Museums in Göttingen, so stellt man fest, dass zwar ein ,Naturalienkabinett’ angekauft wurde, in Zeitschriftenartikeln jedoch vor allem Berichte über das ,Academische Museum’ erschienen. Aus diesem Umstand kann

sche Werke sowie die bereits oben erwähnten Sammlungsgegenstände enthielt. Diese Bestände gelangten jedoch - gemäß der Absprache - erst nach dem Tod Uffenbachs nach Göttingen. Vgl. HARTMANN/FÜCHSEL, Universitäts-Bibliothek, 20, 73.

236 Spätestens für das Jahr 1805 ist belegt, dass sich die Gemäldesammlung der Universität in der zweiten Etage des Gebäudes, in dem auch das Academische Museum untergebracht war, befand. Vgl. FRIEDRICH SAALFELD, Johann Stephan Pütters Versuch einer academischen GelehrtenGeschichte von der Georg-Augustus-Universität zu Göttingen Theil 3: Von 1788-1820, Hannover $1820,425 f$.

237 Vgl. UAG Kur 4 V g 11, Schreiben vom 17. Oktober 1778.

238 Nach 1778 vereinheitlicht sich die Terminologie nicht wesentlich. In den Primärquellen finden sich sowohl verschiedene Diminuitivkomposita von Kabinett als auch unterschiedliche Abwandlungen des Wortes Museum.

239 GeORG Christoph LiCHTENBERG, Etwas vom Academischen Museum in Göttingen, in: Göttinger Taschen Calender. Göttingen 1779, 45-57. 
gefolgert werden, dass die Bezeichnung Museum bewusst gewählt wurde. Nach Hinzuziehung von Verwaltungsquellen kann man ausschließen, dass sich das Academische Museum zum Zeitpunkt der ersten Verwendung dieses Namens durch seine Objekte wesentlich von herkömmlichen Naturaliensammlungen abhob. Plausibler erscheint die These Valters, dass „der Museumsbegriff die Studienfunktion der Kabinette“ ${ }^{240}$ betone. Inwieweit diesbezüglich ein Rückgriff auf das antike Vorbild des Museum Alexandrinum als „Sammelplatz der Gelehrsamkeit““241 bei der Namensgebung für das Academische Museum eine Rolle spielte, ist heute ebenso wenig nachvollziehbar wie auszuschließen: Immerhin war Heyne, der erste Direktor des Museums, Altphilologe. Einen anderen Lösungsansatz bietet ein Eintrag im Zedler zu Kunstkammern: „Kunstkammer / Lat. Museum, Frantz. Cabinet, ist ein zusammengebrachter und wohlgeordneter Vorrath von allerhand Seltenheiten der Kunst / [...] wobey gemeiniglich auch die Seltenheiten der Natur gefüget werden/ dergleichen in Fürstlichen Hof Lagern/ bey grossen Städten/ hohen Schulen/ auch wohl privatHäusern hin und wieder angetroffen/ und von Durchreisenden mit Lust besuchet werden.“ ${ }^{242}$ Demnach könnte es sich beim Wort Museum lediglich um die lateinisierte Form des Wortes Kabinett handeln. Unterstellt man, dass die Namensgebung der neuen Universitätssammlung in bewusster Abgrenzung zu deren vorheriger Bezeichnung als Büttnerschem Naturalienkabinett erfolgte, so wäre dies mit dem Namen Academisches Museum vollzogen - und darüber hinaus ein gewisses Selbstverständnis der Universität zum Ausdruck gebracht. ${ }^{243}$ Eine weitere Möglichkeit für die Wahl des Namens ist, Museum als übergeordneten Begriff bzw. größere Einheit aufzufassen, der mehrere Sammlungen oder auch Abteilungen untergeordnet sind womit gleichzeitig ein bestimmter Ordnungsanspruch impliziert wird. Dafür sprechen einerseits die Aufteilung des Museums, auf die an späterer Stelle noch einzugehen sein wird, und der Titel einer Rechnung aus dem Jahr 1818, in der es heißt: „Verzeichniß der Ausgaben für das Naturalien-Cabinet des königlichen academi-

\footnotetext{
${ }^{240}$ VALTER, Kunst- und Naturaliensammlungen, 37.

241 ZEDLER, Bd 22, Sp. 1376.

242 ZEDLER, Bd 15, Sp. $2143 \mathrm{f}$.

243 Diese These findet sich in ähnlicher Form bei VALTER, Kunst- und Naturaliensammlungen, 35. Dort heißt es: „Auffällig ist jedoch, [...] daß mit dem Museumsbegriff die Verbindung zur Persönlichkeit des Sammlers besonders betont wird.“
} 
schen Museum hieselbst“ “. ${ }^{244}$ Zudem fügt es sich in die allgemeine Tendenz, dass in der Sammlungslandschaft jener Zeit in verstärktem Maße spezialisierte, auf einzelne Bereiche der Naturgeschichte beschränkte Sammlungen entstanden.

Zusammenfassend lässt sich bis hierher feststellen: Das Museum verfügte zwar nur über eine geringe personelle Ausstattung und kein spezifisch für Ausstellungszwecke errichtetes Gebäude, befand sich jedoch - in Hinblick auf das Universitätsleben - an exponierter Stelle. Seine Bestände wurden von einem eigens dazu angestellten, auf diesem Gebiet ausgewiesenen Wissenschaftler geordnet, der dazu berechtigt war, die Objekte in Forschung und Lehre einzusetzen. Das Entstehen des Museums wurde in den Göttingischen Anzeigen von Gelehrten Sachen, ${ }^{245}$ einem führenden Rezensionsorgan dieser Zeit bekannt gemacht. Darüber hinaus wurde verschiedentlich die wenn auch reglementierte - öffentliche Zugänglichkeit des Museums erwähnt. Außerdem ist zu folgern, dass sich das Academische Museum in die Tradition einer aus einer bürgerlichen Privatsammlung entstandenen, späterhin universitär verorteten Sammlung einordnen lässt. Es fügt sich somit in die in Kapitel 1 dargestellte Entwicklung ein und schlägt keinen Sonderweg ein. Allerdings ist hervorzuheben, dass die Ankaufsbestrebungen von Seiten einzelner Professoren ausgingen und das Museum nicht allein aus landesherrlichem Geltungsstreben entstand: Nicht allein Repräsentation, sondern vor allem auch Zweckgebundenheit bzw. wissenschaftliche Einsatzmöglichkeiten und die Intention einer eingeschränkt-öffentlichen Zugänglichkeit spielten eine Rolle. Ob der Gedanke, mit der Einrichtung eines Museums einen Ort für den Austausch von Gelehrten untereinander zu schaffen, ebenfalls intendiert war, bleibt offen.

\footnotetext{
244 UAG Kur 4 V g 20, 76.

245 Vgl. hierzu bspw. Martin GierL, Kompilation und die Produktion von Wissen im 18. Jahrhundert, in: Helmut Zedelmaier/Martin Mulsow (Hg.), Die Praktiken der Gelehrsamkeit in der Frühen Neuzeit, Tübingen 2001, 63-94.
} 


\section{Bestandsveränderungen}

\subsection{Sammlungszugewinne}

Das (systematische) Erweitern bestehender Sammlungen bildet einen integralen Bestandteil musealer Praxis. Grundsätzlich kann man zwei Arten unterscheiden Sammlungszugewinne zu erhalten: Ankauf und Schenkung. Hier lassen sich jedoch weitere feine Unterschiede aufzeigen. Beim Ankauf ist beispielsweise zwischen dem Erwerb angebotener Objekte (oft Nachlässe) und einer Akquisition im engeren Sinne, also einer aktiven Rolle des Museums zu differenzieren. Schenkungen wiederum können in einmalige Schenkungen und regelrechtes Mäzenatentum untergliedert werden. Ein weiteres Unterscheidungskriterium bildet der Anlass der Schenkung. Es kann sich um eine Gabe oder Gegengabe unter Gelehrten handeln oder um einen Nachlass, um Bodenfunde, die an die nächst denkbare Institution abgegeben werden oder um Sammlungsbestände privater Sammler, die diese - je nach Wesen der Gabe - geschlossen in wissende oder forschende Hände geben wollten.

In der Geschichte des Academischen Museums finden sich für all diese Anlässe Beispiele. ${ }^{246}$ Sämtlichen überlieferten Sammlungszugewinnen en detail nachzuspüren würde den Rahmen dieser Arbeit in jeder Hinsicht sprengen. So geht es im Folgenden nicht darum, durch die Ermittlung einzelner Sammlungszugewinne zu einer annähernd vollständigen Rekonstruktion des Gesamtbestandes zu gelangen, ${ }^{247}$ sondern darum, einen Einblick in die Verschiedenartigkeit der Sammlungszugewinne zu geben $^{248}$ und darauf zu verweisen, dass auf diese Weise gesellschaftliche Netzwerke ihre Spuren hinterlassen haben, und dass letztlich jede Gabe ihre eigene kleine Geschichte hat.

Dies sei am Beispiel der (Cook-Forster-)Sammlung illustriert. Der Erwerb dieser Sammlung stellt nicht nur den bei weitem berühmtesten, sondern wohl auch am bes-

${ }^{246}$ Ob es neben dinglichen Gaben auch zu finanziellen Zuwendungen durch private Spender kam, ist nicht bekannt.

247 Vgl. hierzu das ambitionierte Projekt, die inzwischen auf verschiedene Nachfolgeinstitutionen verteilten Sammlungsgegenstände der früheren Königlich Dänischen Kunstkammer in einem Katalogband zusammenzuführen und so in gewisser Hinsicht ein virtuelles Museum mit dem Bestand von 1737 entstehen zu lassen. Vgl. BENTE GundESTRuP, Det kongelige danske Kunstkammer 1737 = The Royal Danish Kunstkammer 1737 [1] 3 binder / 3 volumes, København 1991-1995.

248 Soweit die Disparität des Quellenkorpus' dies zulässt. 
ten dokumentierten Sammlungszuwachs des Academischen Museums dar. Die dieser Sammlung zuzurechnenden Objekte gelangten in mehreren Etappen nach Göttingen, wesentlich jedoch in den Jahren 1781/82. Eine ins Detail gehende Analyse der Erwerbsgeschichte lieferte jüngst Urban, ${ }^{249}$ so dass deren Beschreibung an dieser Stelle kurz gehalten werden kann: Im August 1781 richtete Blumenbach ein Schreiben an die „Königlich Gros Brittannische zur Churfürstlich Braunschweig-Lüneburgischen Landes Regierung Höchst verordnete Herren Geheimde Räthe“, in dem er darum bat,

ob nicht durch Hochdero Gnädiges Vorwort beÿ Ihro Königlicher Majestät etwas von dem Ueberfluße ausländischer Natürlicher Merkwürdigkeiten, welche besonders durch die, auf Ihro Majestät Befehl neuerlich vollzognen Reisen ${ }^{250}$ um die Welt in größter Menge gesammelt worden, für das academische Museum zu erhalten stünde ${ }^{251}$

Blumenbachs Gesuch hatte Erfolg. Georg III. zeigte sich großzügig und gewährte dem Museum die gewünschte „Sammlung von Seltenheiten“. ${ }^{252}$ Für den Preis von etwas mehr als $100 £$ Sterling ${ }^{253}$ erhielt das Museum am 2. August 1782349 Objekte, v.a. Ethnographica, die vornehmlich von der dritten Weltumsegelung Cooks (Juli 1776 bis Oktober 1780) stammten, und zu denen noch eine detaillierte Auflistung des Zwischenhändlers George Humphrey existiert. ${ }^{254}$ Bis zu diesem Punkt waren an dem Erwerbsvorgang Blumenbach, König Georg III. und der Zwischenhändler George Humphrey beteiligt. Genaueres Hinsehen zeigt jedoch die Beteiligung weiterer Personen, sowie das Beziehungsgeflecht, das den Sammlungserwerb in dieser Form erst möglich machte. An erster Stelle ist hier wohl die politische Konstellation zu nennen. Hannover wurde seit 1714 in Personalunion mit England regiert und sowohl Georg III. als auch schon Georg II., der Gründer der Georgia Augusta, hatten ihre Gewogenheit der Universität gegenüber mehrfach erwiesen. Insofern schien es Blumen-

249 Vgl. URBAN, Erwerbungsgeschichte, 56-85.

250 Dies bezieht sich auf die drei Weltumsegelungen James Cooks: 1. Reise: 1768-1771, 2. Reise: 1772-1775, 3. Reise: 1776-1780. Kapitän Cook wurde mit Teilen seiner Mannschaft am 14. Februar 1779 von Teilen der indigenen Bevölkerung auf Hawaii getötet.

251 AVkG, Akten 1: Altakten lose, hier: Erwerbsunterlagen: Cook-Sammlung 1781/1782, Schreiben vom 27. August 1781 an die Königlich Gros Brittannische zur Churfürstlich BraunschweigLüneburgischen Landes Regierung Höchst verordnete Herren Geheimde Räthe, 1r u. v.

${ }^{252}$ Vgl. AVkG, Akten 1: Altakten lose, hier: Erwerbsunterlagen: Cook-Sammlung 1781/1782, Post Scriptum an die „Geheimten Räte in Hannover“ vom 24. Dezember 1781.

253 Die genaue Summe von £105 14s 10d betrug ,zur damaligen Zeit in deutscher Währung um 560 Reichstaler. Die Summe entsprach damit in etwa dem festen Jahresgehalt eines besser besoldeten Göttinger Professors. Die Mehrzahl der hiesigen Hochschullehrer, so auch Blumenbach und Lichtenberg, mußten sich zu jener Zeit mit 250 bis 450 Reichstalern begnügen.“ Zit. nach URBAN, Erwerbungsgeschichte, $65 f$.

254 Die wissenschaftsgeschichtliche Bedeutung des so genannten Humphrey-Katalogs ist nicht zu überschätzen. Vgl. URBAN, Erwerbungsgeschichte, 62. 
bach nicht abwegig, sich um Objekte von englischen Forschungsreisen zu bemühen. Sodann ist es hilfreich zu wissen, dass diesem großen Zuwachs kleinere Gaben vorausgingen. Bereits im Catalogus Musei Academici lassen sich unter der Rubrik „Artefacten“ Objekte nachweisen, die auf Grund ihrer Beschaffenheit der ersten Weltumsegelung Cooks (September 1768 bis Juli 1771) zuzuordnen sind. ${ }^{255}$ Zeitpunkt und Umstände ihres Erwerbs sind jedoch nicht rekonstruierbar. Auch später gelangten vereinzelt Objekte aus der Südsee nach Göttingen, von denen zumindest drei sicher auf Georg Forster (1754-1794) zurückgehen. Johann Reinhold Forster (17291798) und dessen damals 18-jähriger Sohn Georg hatten James Cook als Naturforscher auf dessen zweiter Reise (Juli 1772 bis Juli 1775) begleitet. Dadurch verfügten sie selbst über seltene ethnographische Objekte und Naturalien und, wie nur wenige andere Menschen, über Kenntnisse über die besuchten Völker und Regionen. Daneben dürften ihnen auch Informationen über die Cook'schen Reisen selbst, sowie - zumindest in begrenztem Umfang - Kenntnisse über die Organisation der Reisen und der daran beteiligten Personen zugekommen sein. Dies war insofern für das Academische Museum von Belang, als Georg Forster im Jahr 1778 eine Stellung als Professor für Naturkunde am Carolinum in Kassel annahm. ${ }^{256}$ In der Folgezeit suchte er des Öfteren Göttingen auf - zunächst vor allem wegen der Bibliothek, die damals bereits einen hervorragenden Ruf genoss, und bald auch wegen der Kontakte zu anderen Gelehrten. Bereits bei seinem ersten Besuch in Göttingen schloss er Bekanntschaft mit Heyne, Blumenbach, Büttner und anderen dortigen Professoren. Besonders mit Lichtenberg sollte ihn eine enge Freundschaft verbinden. ${ }^{257}$ Große Verehrung brachte er Heyne entgegen, mit dem er später in verwandtschaftliche Beziehung treten sollte, indem er sich 1785 mit dessen Tochter Therese vermählte. Jedenfalls offenbart sich auf diese Weise eine direkte Verbindung zwischen den Reisen Cooks und der Göttinger Gelehrtenschaft. Es ist mehr als wahrscheinlich, dass Forster bei diesem Sammlungserwerb eine Schlüsselrolle als Mittler oder Impulsgeber zukam. Blumenbachs oben zitiertes Schreiben gelangte also in erwartet wohlwollende Hän-

255 Vgl. URBAN, Erwerbungsgeschichte, 57.

${ }^{256}$ Forster blieb fünf Jahre in Kassel. 1779 wurde er Prorektor des Carolinums, 1784 nahm er einen Ruf nach Wilna an.

257 So gaben sie bspw. gemeinsam das Göttingische Magazin der Wissenschaften und Litteratur heraus. Vgl. GEORG CHRISTOPH LiCHTENBERG/GEORG FORSTER (Hg.), Göttingisches Magazin der Wissenschaften und Litteratur, Göttingen 1780-1785. 
de: Die von englischer Seite mit der Auswahl der Cook-Objekte betraute Person, der Geheime Justizrat Christian Heinrich von Hinüber, war zu dem Zeitpunkt bereits mehr als zwanzig Jahre im Dienste des Hofes in London tätig und hatte zuvor selbst in Göttingen studiert. Außerdem war zum Zeitpunkt des Objekttransfers sein Sohn in Göttingen immatrikuliert. Christian Heinrich von Hinüber nutzte die Gelegenheit, in einem Schreiben bezüglich der Fortschritte des Zusammenstellens der für Göttingen gedachten Sammlungen seinen Sohn Blumenbach anzuempfehlen. ${ }^{258}$ Als Gegenleistung für die erwartete Protektion seines Sohnes durch Blumenbach scheint sich von Hinüber nicht nur um eine schnelle Abwicklung des Geschäfts, sondern auch darum bemüht zu haben, dem Göttinger Museum weitere wertvolle, die Sammlung ergänzende Stücke zu verschaffen. ${ }^{259}$ Hier offenbart sich ein Einblick in das komplexe System des frühneuzeitlichen Gabentausches in Verbindung mit wissenschaftlichen, politischen und ökonomischen Beziehungen. Blumenbach und Forster waren hierin ebenso eingebunden wie Georg III. oder auch von Hinüber.

Etwas zu Unrecht überlagert die Erwerbung der Cook-Sammlung in ihrer Bedeutung andere, ebenfalls beachtliche, zeitnah erfolgte Sammlungszugewinne. Die regelmäßigen Geschenksendungen des Barons von $\operatorname{Asch}^{260}$ an seine Alma Mater, ${ }^{261}$ die vor allem Mineralien, aber auch Naturalien und Ethnographica enthielten, sind hier ebenso zu nennen, wie der Ankauf der Stelznerschen Mineraliensammlung (1782), die den Grundbestand des Academischen Museums systematisch ergänzte. Dabei war die letztgenannte Sammlung so umfangreich, dass ihre Einarbeitung „ein gänzliches umrangiren und völlig neue Anordnung des Musei erfordert: so daß von so vielen tausend Stücken kein einziges an seiner vorigen Stelle [hat] bleiben können““. ${ }^{262}$ Dennoch wurde diesem Ankauf weder von den Zeitgenossen noch von der Forschung ein ähnliches Maß an Aufmerksamkeit zuteil, wie den von den Weltumsege-

${ }^{258}$ Vgl. AVkG, Akten 1: Altakten lose, hier: Erwerbsunterlagen: Cook-Sammlung 1781/1782, Schreiben vom 21. Dezember 1781.

259 Inwiefern Blumenbach den jungen von Hinüber tatsächlich unter seine Fittiche nahm ist ohne tiefer gehende Recherchen nicht nachvollziehbar. Blumenbach brachte seine Gewogenheit von Hinüber gegenüber jedenfalls dadurch zum Ausdruck, dass er ihm eine Ausgabe seines Handbuchs der Naturgeschichte zukommen ließ. Ob dies eine Geste der Zuneigung oder bloß eine Höflichkeitsbezeugung war, ist ungewiss.

260 Vgl. UAG Kur 4 V g 9.

261 Vgl. PlischKe, EthnOGRAPHische Sammlung, 26. Von Asch hatte in Göttingen bei Albrecht von Haller studiert und promoviert.

${ }^{262}$ UAG Kur 4 V g 9, Schreiben vom 26. August 1782. 
lungen James Cooks (1728-1779) stammenden Sammlungsgegenständen. Ein mögliches Erklärungsmuster hierfür bietet der Bedeutungsgehalt dieser Gegenstände. Mit Pomian gesprochen handelt es sich um Semiophoren mit einem besonders hohen symbolischen Wert, der durch die Überlagerung mehrerer Bedeutungsebenen potenziert wurde: Die Objekte hatten nicht nur Seltenheitswert in Europa ${ }^{263}$ und wirkten auf hiesige Betrachter fremd, sie gaben zudem Zeugnis von den Taten der großen Seefahrernation England und stellen zudem noch einen Erinnerungsort für Cook dar, der bei ihrem Erwerb ums Leben kam. Außerdem - und das wird leicht übersehen erhielt durch sie die indigene Bevölkerung der bereisten Erdteile in der Wahrnehmung ,der’ Europäer eine Geschichtlichkeit.

Die Bedeutung dieser Sammlung liegt nicht allein in der Verknüpfung von Sichtbarem und Unsichtbarem durch die materialen Eigenschaften der Objekte, sondern für die Geschichte des Museums - auch darin, dass damit ein bis dahin nur schwach vertretenes Sammlungsfeld erheblich gestärkt wurde, woraufhin sich nach Pütter eine eigenständige, die ethnographische, Abteilung herausbildete. ${ }^{264} \mathrm{Zu}$ diesem Zeitpunkt befand sich die Völkerkunde ${ }^{265}$ als eigene Disziplin noch in der Etablierungsphase; durch den Erwerb der Cook-Sammlung erhält die Göttinger Völkerkunde in dieser Hinsicht eine exponierte Stellung. Überdies ist die Erwerbsgeschichte dieser Sammlung ausnehmend gut überliefert und erhält dadurch einen hohen wissenschaftshistorischen Wert. Außerdem stellt sie ein hervorragendes Beispiel für eigene Akquisitionstätigkeit des Museums dar. ${ }^{266}$

Unter den weiteren dauerhaften Sammlungszugewinnen des Academischen Museums nahmen der Erwerb des Forster-Nachlasses (1799), der eine wichtige Ergänzung zu den Beständen der Cook- Expedition bildete, und der Kauf des Blumenbach-

263 „Rund 2000 Ethnographica der Cook-Reisen sind weltweit in verschiedenen Sammlungen bekannt und dokumentiert. [...] Von diesen befinden sich etwa 500, also etwa ein Viertel, in der Göttinger Sammlung.“ ADRIENNE L. KAEPPLER, Die Göttinger Sammlung im internationalen Kontext, in: Hauser-Schäublin/Krüger (Hg.), James Cook, 86-93, 86; ferner: MANFRED URBAN, 200 Jahre Göttinger Cook-Sammlung, Göttingen 1982, 19-20.

264 Vgl. PLISCHKE, Ethnographische Sammlung, 13.

265 Die Begriffe Ethnographie und Völkerkunde finden sich im deutschen Sprachraum erstmals bei Gatterer und Schlözer um 1770. Vgl. HAN F. VERMEULEN, Gatterer und Schlözer: Völkerkunde in der Aufklärung in Göttingen in den Jahren 1770, Leiden 1990.

266 Blumenbach akquirierte jedoch auch in kleinerem Maßstab. Ein im Archiv der Göttinger Akademie der Wissenschaften erhaltenes Schreiben gibt darüber Auskunft, dass er anhand einer Durchsicht der GGA Objekte ermittelte, die durch Schenkungen an die Akademie gelangt waren und die er dann für das Museum erbat. Vgl. AAW Scient 236. 
Nachlasses (1840), insbesondere die darin enthaltene Schädelsammlung, eine herausragende Stellung ein. Durch sie gelangten wichtige, die Sammlung bereichernde Objekte ans Museum. Dennoch soll an dieser Stelle nicht näher auf sie eingegangen werden. Vielmehr soll anhand der folgenden Auflistung eine Übersicht über die in den Akten des Göttinger Universitätsarchivs eindeutig nachweisbaren dauerhaften Sammlungszugewinne gegeben werden. Die folgende Aufstellung lässt die napoleonische Ära aus, da ein Großteil der Ära aus Kassel nach Göttingen überführten Sammlungsstücke, nach der Auflösung des Königreichs Westphalen wieder zurückgegeben wurden. Vereinzelt (s. Tabelle) wurde bei bekannteren Zugewinnen auch auf andere Quellen zurückgegriffen. Ein über den gesamten Untersuchungszeitraum geführtes chronologisches oder systematisches Eingangsverzeichnis ist, falls es je existierte, nicht erhalten.

Vorab noch eine quellenkritische Bemerkung: Die Sammlungszugewinne in ihrer Gesamtheit zu erfassen stellt nicht nur deshalb ein Problem dar, weil unklar ist, ob a) tatsächlich alle Zugänge verzeichnet wurden, und b) in welchem Umfang Akten kassiert wurden oder aus anderen Gründen nicht erhalten sind. Vielmehr stellt es ein Problem dar, die Akten mehr als orakelhaft zum Sprechen zu bringen. Dies sei kurz an einem Beispiel erläutert. Das sehr umfangreiche ${ }^{267}$ Aktenkonvolut UAG Kur 4 V g 20 enthält vor allem Rechnungen und Rechnungsbelege, die - zumindest für bestimmte Zeiträume - sehr ausführlich über die für das Museum aufgewendeten Kosten Auskunft geben. Unter diesen Belegen finden sich etliche detaillierte Rechnungen des Beutlers und Handschuhmachers Georg Klener, der wie die Rechnungen zeigen, für das Ausstopfen, insbesondere von Vögeln, zuständig war. Allerdings geht aus den Rechnungen nicht hervor, woher diese Vögel stammten. Handelte es sich um Neuerwerbungen, die zu Ausstellungszwecken aufgearbeitet wurden und bei denen Klener vielleicht eine Vermittlerrolle zukam? Oder handelte es sich um die sukzessiv erfolgte Aufbereitung von Altbeständen des Museums? Ähnlich diffizil verhält es sich mit dem auf den einzelnen Jahresabrechnungen erscheinenden Punkt „Obermedizinalrath Blumenbach Auslagen“. Diese bewegen sich in einem Kostenrahmen, der durchaus den Ankauf von Sammlungsstücken einschließen könnte, enthalten aber in

267 Für den Zeitraum 1814 bis 1840 umfasst es ca. 250 Seiten. Das gesamte Konvolut weist mehr als 1600 beschriebene Seiten auf. 
der Regel keine Detailinformationen. Eine Einzelauflistung aus dem Jahr 1818 bestätigt, dass Blumenbachs Auslagen tatsächlich auch Sammlungsgegenstände und nicht nur für Frachtkosten und dergleichen beinhalteten. ${ }^{268}$

\begin{tabular}{|c|c|c|}
\hline \multicolumn{3}{|c|}{ Übersicht der Sammlungszugewinne 1773-1840 } \\
\hline Zeitpunkt & Art und Herkunft der Objekte & Belegstellen \\
\hline 1773 & $\begin{array}{l}\text { Ankauf des Büttnerschen Naturalienkabinetts als } \\
\text { Grundbestand des Academischen Museums }\end{array}$ & GGA 1773, 1114 \\
\hline 1777 & $\begin{array}{l}\text { Schenkung der sog. Schlüterschen Sammlung } \\
\text { durch Georg III. Diese Sammlung wurde zuvor in } \\
\text { der königlichen Bibliothek in Hannover aufbe- } \\
\text { wahrt und enthielt v.a. Erzstufen, Drusen, und Sil- } \\
\text { berstufen. }\end{array}$ & $\begin{array}{l}\text { UAG Kur } 4 \text { V g 6, } \\
\text { AVkG, Akten 1- } \\
\text { Altakten lose, Alte } \\
\text { Dokumente 19. Jh., } \\
\text { J.F. Blumenbach } \\
\text { über die „Natura- } \\
\text { liensammlung des } \\
\text { academischen Mu- } \\
\text { sei“, 28.10.1808, } \\
\text { UAG Kur } 4 \text { V g } 7 \\
\end{array}$ \\
\hline 1778 & $\begin{array}{l}\text { Naturalien-Geschenke der verwitweten Fürstin von } \\
\text { Waldeck }\end{array}$ & UAG Kur 4 V g 4 \\
\hline 1778 & $\begin{array}{l}\text { Mai: } \\
\text { - Geschenksendung des Barons von Asch, } 58 \\
\text { Nummern, ausschließlich Mineralien, darunter } \\
\text { viele Abarten von gediegenem Gold und Silber } \\
\text { August: } \\
\text { - Geschenksendung des Barons von Asch; Ins- } \\
\text { gesamt } 84 \text { Nummern, davon } 83 \text { Mineralien, } \\
\text { viel aus Sibirien, teils aus Finnland } \\
\text { November: } \\
\text { - Vom Herrn Treische, Kaufmann in Erfurt, ins- } \\
\text { gesamt etwa } 20 \text { Nummern, vor allem Minera- } \\
\text { lien, einige Naturalien. } \\
\text { - Geschenk von Herrn Gottlieb aus dem Spon- } \\
\text { heimischen; insges. } 20 \text { Nummern, Mineralien } \\
\text { ohne Datierung, wahrscheinlich 1778/1779: } \\
\text { - Geschenk von Louis Sulzer, Negociant zu Neu } \\
\text { - Orleans; Naturalien } \\
\text { - Geschenk von Georg Forster in Kassel; drei } \\
\text { Ethnographica } \\
\text { - Geschenk von Prof. Boffart [?] in Barby; drei } \\
\text { - Gummern, Mineralien und eine Versteinerung } \\
\text { - Geschenk der verwitweten Fürstin von Wal- } \\
\text { Geschenk von Herrn Friedrichs, Negociant auf } \\
\text { Cap d'Orange in Guyana; Ethnographica }\end{array}$ & $\begin{array}{l}\text { UAG Kur } 4 \text { V g 9, } \\
\text { 47f, 49f, 64r, 64v, } \\
\text { 65r, 65v, 66r, 66v }\end{array}$ \\
\hline
\end{tabular}

${ }^{268}$ Vgl. UAG Kur 4 V g 20, 60. 


\begin{tabular}{|c|c|c|}
\hline Zeitpunkt & Art und Herkunft der Objekte & Belegstellen \\
\hline & $\begin{array}{l}\text { - Geschenk von Kapitän Duplat; „Drey ohnweit } \\
\text { Hameln ausgegrabene altteutsche Urnen.“ } \\
\text { - Geschenk von Professor Büttner; } 14 \text { Num- } \\
\text { mern, Mineralien (Halbedelsteine) } \\
\text { - Geschenk von Amtsschreiber Niemann zu } \\
\text { Lauenstein; } 8 \text { Objekte, Mineralien (v.a. Schwe- } \\
\text { fel) } \\
\text { - „Vom Herrn - - - - . Eines Tatterherren Staats- } \\
\text { Peitsche mit Silber durchwürkt ein dergleichen } \\
\text { Quästchen pp.“ } \\
\text { - Geschenk von Madame Deneken aus Bremen; } \\
\text { 27 Nummern, wohl ausschließlich Naturalien } \\
\text { - Geschenk von Dr. Sulzer aus Gotha; drei Ob- } \\
\text { jekte in Spiritus } \\
\text { Geschenk der Fürstin von Nassau-Usingen. } \\
\text { „Nadell des großen Diamanten aus der Spitze } \\
\text { der französischen Crone oder des sogenannten } \\
\text { Pitts, von Crystall de roche“ }\end{array}$ & \\
\hline 1779 & $\begin{array}{l}\text { Mai: } \\
\text { - Geschenksendung des Barons v. Asch; Insge- } \\
\text { samt } 16 \text { Nummern, Naturalien und Mineralien } \\
\text { Juni: } \\
\text { - Geschenksendung des Barons v. Asch; insge- } \\
\text { samt } 92 \text { Nummern, v.a. Mineralien (einschl. } \\
\text { Metallen und Halbedelsteinen), Naturalien } \\
\text { August: } \\
\text { - Geschenksendung des Barons von Asch; insge- } \\
\text { samt } 85 \text { Nummern, etwas mehr als die Hälfte } \\
\text { Mineralien, aber auch zahlreiche Naturalien u. } \\
\text { Ethnographica bzw. nach älterem Verständnis } \\
\text { z.T. Curiosita, so z.B.: zwei Bezoare. Darüber } \\
\text { hinaus Teile einer Ägyptischen Mumie } \\
\text { September: } \\
\text { - Geschenksendung des Barons von Asch; insge- } \\
\text { samt } 102 \text { Nummern, überwiegend Mineralien, } \\
\text { einige Muscheln und Korallen, verschiedenar- } \\
\text { tige Versteinerungen, zwei Ethnographica. } \\
\text { Oktober: } \\
\text { • Geschenksendung des Barons von Asch; insge- } \\
\text { samt } 55 \text { Nummern, überwiegend Mineralien } \\
\text { November: } \\
\text { - Geschenksendung des Barons von Asch; insge- } \\
\text { samt } 22 \text { Nummern, hauptsächlich Mineralien, } \\
\text { darunter aber auch „zwey Heuschrecken“ und } \\
\text { eine Sammlung getrockneter Pflanzen }\end{array}$ & $\begin{array}{l}\text { UAG Kur } 4 \text { V g 9; } \\
\text { 51, 52f, 56, 54f, 57, } \\
58\end{array}$ \\
\hline
\end{tabular}




\begin{tabular}{|c|c|c|}
\hline Zeitpunkt & Art und Herkunft der Objekte & Belegstellen \\
\hline 1780 & $\begin{array}{l}\text { von Juni ab: } \\
\text { - Bemühungen um die Vervollständigung der } \\
\text { Sammlung durch Erzstufen aus dem Harz; Der } \\
\text { Verhandlungsverlauf war günstig, jedoch ist } \\
\text { der Akte nicht zu entnehmen, ob und in wel- } \\
\text { chem Umgang es tatsächlich zu einer Samm- } \\
\text { lungserweiterung kam. } \\
\text { August: } \\
\text { - Geschenksendung des Barons v. Asch; Insge- } \\
\text { samt etwa 15 Objekte, Mineralien, Naturalien, } \\
\text { Ethnographica } \\
\text { September: } \\
\text { - Geschenksendung des Baron v. Asch; Insge- } \\
\text { samt 149 Nummern, v.a. Mineralien, aber auch } \\
\text { eine Kokosnuss und } 3 \text { alte russische Münzen } \\
\text { Oktober: } \\
\text { - Geschenksendung des Barons v. Asch; insge- } \\
\text { samt } 8 \text { Nummern, wohl nur Mineralien } \\
\text { Geschenk von Professor Hollmann; 23 Num- } \\
\text { mern, wohl v.a. Mineralien } \\
\text { November: } \\
\text { - Geschenk des Rußisch-Kayserl. Herrn Gehei- } \\
\text { men Rath Iwan Iwanowitz; „Ein besonderer } \\
\text { Kasten mit 12. Schieben von Mahagony Holz } \\
\text { worinn 432. verschiedene Sorten Sibirische } \\
\text { Jaspis Achate, Granite u. s. w. befindlich.“ } \\
\text { Dezember: } \\
\text { Geschenksendung des Barons v. Asch; insge- } \\
\text { samt etwa 37 Objekte, Naturalien, Petrefakten, } \\
\text { Mineralien }\end{array}$ & $\begin{array}{l}\text { UAG Kur } 4 \text { V g 7; } \\
\text { UAG Kur } 4 \text { V g 9, } \\
\text { 59, 60f, 62, 63, 64r, } \\
66 v\end{array}$ \\
\hline 1781 & $\begin{array}{l}\text { Februar: } \\
\text { • Schenkung einer ägyptischen Mumie durch } \\
\text { den König von Dänemark } \\
\text { März: } \\
\text { • „Gnädigst bewilligter Ankauf; } 23 \text { Nummern, } \\
\text { Mineralien, danach noch: 1. Ein Mumien Fus, } \\
\text { 2. Eine Crystalldruse mit Wassertropfen [?] aus } \\
\text { der Bulgarey.“ }\end{array}$ & $\begin{array}{l}\text { UAG Kur } 4 \text { V g } 8 \text {, } \\
\text { GGA } 24 \text { (1781), } \\
\text { 185, UAG Kur } 4 \text { V } \\
\text { g } 967 \text { r }\end{array}$ \\
\hline 1782 & $\begin{array}{l}\text { Ankauf des Naturalien-Kabinetts des Oberberg- } \\
\text { meisters Stelzner; enthielt vor allem Fossilien aus } \\
\text { dem Harz und Silberstufen }\end{array}$ & $\begin{array}{l}\text { UAG Kur } 4 \text { V g 9, } \\
\text { AVkG, Akten 1- } \\
\text { Altakten lose, Alte } \\
\text { Dokumente 19. Jh., } \\
\text { J.F. Blumenbach } \\
\text { über die „Natura- } \\
\text { liensammlung des } \\
\text { academischen Mu- } \\
\text { sei“, 28.10.1808. }\end{array}$ \\
\hline
\end{tabular}




\begin{tabular}{|c|c|c|}
\hline Zeitpunkt & Art und Herkunft der Objekte & Belegstellen \\
\hline $1781 / 82$ & Erwerb der Cook-Sammlung & $\begin{array}{l}\text { AVkG, Altakten } \\
\text { (lose Blattsamm- } \\
\text { lung), Erwerbsun- } \\
\text { terlagen Cook-Slg }\end{array}$ \\
\hline 1783 & $\begin{array}{l}\text { Nachtrag einiger Stücke der ehemaligen Büttner- } \\
\text { schen Sammlung } 269\end{array}$ & \\
\hline 1791 & $\begin{array}{l}\text { Der Ankauf der zoologischen Sammlung des Me- } \\
\text { dizinal-Rats Brandis zu Hildesheim. }\end{array}$ & UAG Kur 4 V g 13 \\
\hline 1791 & $\begin{array}{l}\text { „Zukauf der Sammlungen des Dr. Schencker zu } \\
\text { Hildesheim“; entomologische Sammlungsstücke }\end{array}$ & $\begin{array}{l}\text { AVkG, Akten 1- } \\
\text { Altakten lose, Alte } \\
\text { Dokumente 19. Jh., } \\
\text { J.F. Blumenbach } \\
\text { über die „Natura- } \\
\text { liensammlung des } \\
\text { academischen Mu- } \\
\text { sei“, 28.10.1808. }\end{array}$ \\
\hline 1794 & $\begin{array}{l}\text { „Die Übersendung eines im Amte Ottersberg im } \\
\text { Moor gefundenen alten Kahns für das Museum“ }\end{array}$ & UAG Kur 4 V g 14 \\
\hline 1799 & Erwerb des Forster-Nachlasses & $\begin{array}{l}\text { AVkG, Altakten } \\
\text { (lose Blattsamm- } \\
\text { lung), J.R. Forster }\end{array}$ \\
\hline 1817 & $\begin{array}{l}\text { Ankauf von Mineralien und einem zoologischen } \\
\text { Objekt }\end{array}$ & $\begin{array}{l}\text { UAG Kur } 4 \text { V g 20, } \\
84,86\end{array}$ \\
\hline 1818 & $\begin{array}{l}\text { März: } \\
\text { - Ankauf verschiedener ausländischer, insbeson- } \\
\text { dere ungarischer Mineralien von Stufenhändler } \\
\text { Moraweck aus Wien } \\
\text { • Ankauf eines monströsen Kalbes } \\
\text { August: } \\
\text { - Schenkung einer Kiste Mineralien durch Fr. } \\
\text { Zipser zu Neusohl } \\
\text { September: } \\
\text { • Ankauf „unreife[r] Drillinge“ } \\
\text { Oktober: } \\
\text { - Schenkung eines vollständigen Giraffenfells } \\
\text { durch Pastor Hesse }\end{array}$ & $\begin{array}{l}\text { UAG Kur } 4 \text { V g 20, } \\
\text { 60r }\end{array}$ \\
\hline 1819 & $\begin{array}{l}\text { Januar: } \\
\text { • Mineralienlieferung von Stufenhändler Geißler } \\
\text { ohne nähere Datierung: } \\
\text { - Von Pastor Hesse zu Nienburg geschenkte } \\
\quad \text { zoologischen Seltenheiten. }\end{array}$ & $\begin{array}{l}\text { UAG Kur } 4 \text { V g 20, } \\
\text { 66; UAG Kur } 4 \text { V g } \\
23\end{array}$ \\
\hline
\end{tabular}

2691773 hatte die Universität nicht die komplette Sammlung Büttners erhalten: „Nach dem Vertrag vom Mai 1773 hatte Büttner Doppelstücke seiner Sammlung in Verwahrung behalten, um sie auf dem Wege des Tausches ,zum Besten der Sammlung' verwenden zu können. Nun, wo er Göttingen verlassen wollte - und dieser Fall war im Vertrag nicht vorgesehen -, glaubte er, diese Stücke und andere, die er noch nicht abgeliefert hatte, zu seiner weiteren Verfügung zu haben. Durch Heynes Einwirken gelang es, Büttner zur Herausgabe aller Materialien zu veranlassen, und am 10. Oktober 1783 erhielt Büttner seine Dienstentlassung.“ PLISCHKE, Ethnographische Sammlung, 12. 


\begin{tabular}{|c|c|c|}
\hline Zeitpunkt & Art und Herkunft der Objekte & Belegstellen \\
\hline 1823 & $\begin{array}{l}\text { - Ankauf von neun Gipsabgüssen aus den } \\
\text { Sammlungen des Louvre in Paris } \\
\text { - Die bei Mulsum im Lande Wursten im Moor } \\
\text { aufgefundenen Altertümer von Gold. }\end{array}$ & $\begin{array}{l}\text { UAG Kur } 4 \text { V g 24; } \\
\text { UAG Kur } 4 \text { V g } 26\end{array}$ \\
\hline 1827 & $\begin{array}{l}\text { - Ankauf der Naturalien-Sammlung des verstor- } \\
\text { benen Hofrats Ebel (v.a. zoologische Objekte). } \\
\text { - Erhalt des Kataloges des Naturalien-Kabinetts } \\
\text { des verstorbenen John Raye aus Amsterdam. }\end{array}$ & $\begin{array}{l}\text { UAG Kur } 4 \text { V g 28; } \\
\text { UAG Kur } 4 \text { V g } 30\end{array}$ \\
\hline 1829 & $\begin{array}{l}\text { Aus dem Amt Esens wurden dem Museum beim } \\
\text { Torfgraben aufgefundene } 10 \text { Gold- und } 32 \text { Silber- } \\
\text { münzen zur Aufbewahrung übersandt; die dadurch } \\
\text { entstandenen Kosten beliefen sich auf einen (ge- } \\
\text { setzlich festgelegten) Finderlohn in der Höhe des } \\
\text { halben Metallwertes, den die Universitätskasse } \\
\text { gewährte. }\end{array}$ & UAG Kur 4 V g 32 \\
\hline 1836 & $\begin{array}{l}\text { Ankauf (Finderlohn) der zu Fickmühlen, Amt Be- } \\
\text { derkesa, gefundenen altrömischen Silbermünzen. } \\
\text { ( } 47 \text { Münzen in einem tönernen Gefäß. Das Gefäß } \\
\text { wurde der ethnographischen Abt. des Museums } \\
\text { zugeführt, die Münzen an Reuß für die Münz- } \\
\text { Sammlung übergeben. }\end{array}$ & UAG Kur 4 V g 36 \\
\hline 1837 & $\begin{array}{l}\text { Ankauf von Tieren und Insekten, u.a. eines } \\
\text { schwarzen Storchs und Raupen }\end{array}$ & $\begin{array}{l}\text { UAG Kur } 4 \text { V g 20, } \\
142\end{array}$ \\
\hline $1837 / 38$ & Ankauf von Säugetierbälgen und Skeletten & $\begin{array}{l}\text { UAG Kur } 4 \text { V g 20, } \\
131\end{array}$ \\
\hline 1838 & $\begin{array}{l}\text { Juni } \\
\text { - Ankauf von Tier-Skeletten } \\
\text { Juli: } \\
\text { - Ankauf eines viriginischen Iltis, eines ameri- } \\
\text { kanischen Seehunds und eines amerikanischen } \\
\text { Nerz’ } \\
\text { August: } \\
\text { - Ankauf von zoologischen Objekten (Salaman- } \\
\text { der, Frosch) } \\
\text { September: } \\
\text { • Ankauf von „Walfischteilen“ aus Bremen } \\
\text { Oktober: } \\
\text { - Ankauf zoologischer Objekten (Lachs, Dachse) } \\
\text { November: } \\
\text { - Ankauf javanischer Säugetieren und Amphi- } \\
\text { bien aus der Colinaenschen[?] Sammlung in } \\
\text { Wiesbaden } \\
\text { - Geschenk von Dr. Hahn aus Hannover, beste- } \\
\text { hend aus „Seethieren von Norderney“ } \\
\text { Ankauf von Schafembryonen; weiteres für } \\
\text { zoologische Abteilung }\end{array}$ & $\begin{array}{l}\text { UAG Kur } 4 \text { V g 40; } \\
\text { UAG Kur } 4 \text { V g 41; } \\
\text { UAG Kur } 4 \text { V g 20; } \\
\text { 131, 139, 140, 155, } \\
\text { 174, 185 }\end{array}$ \\
\hline
\end{tabular}




\begin{tabular}{|c|c|c|}
\hline Zeitpunkt & Art und Herkunft der Objekte & Belegstellen \\
\hline & $\begin{array}{l}\text { Dezember: } \\
\text { - Ankauf eines Hais } \\
\text { ohne nähere Datierung } \\
\text { - Im Ihlsmoore, Gericht Delm, aufgefundene } \\
\text { und für das Museum angekaufte Altertümer: } \\
\text { eine Axt und zwölf kleinere Werkzeuge aus } \\
\text { bronzeartigem Material. } \\
\text { - Naturaliengeschenke an die zoologische Abtei- } \\
\text { lung des Museums (ohne Herkunftsangabe) } \\
\text { - Ankauf synoptischer Tabellen des Tierreichs } \\
\text { - Ankauf eines Bogens und einer Decke aus } \\
\text { Neuseeland }\end{array}$ & \\
\hline $1838 / 1839$ & Ankauf von Mineralien von der Witwe Geißler & $\begin{array}{l}\text { UAG Kur } 4 \text { V g 20, } \\
152\end{array}$ \\
\hline 1839 & 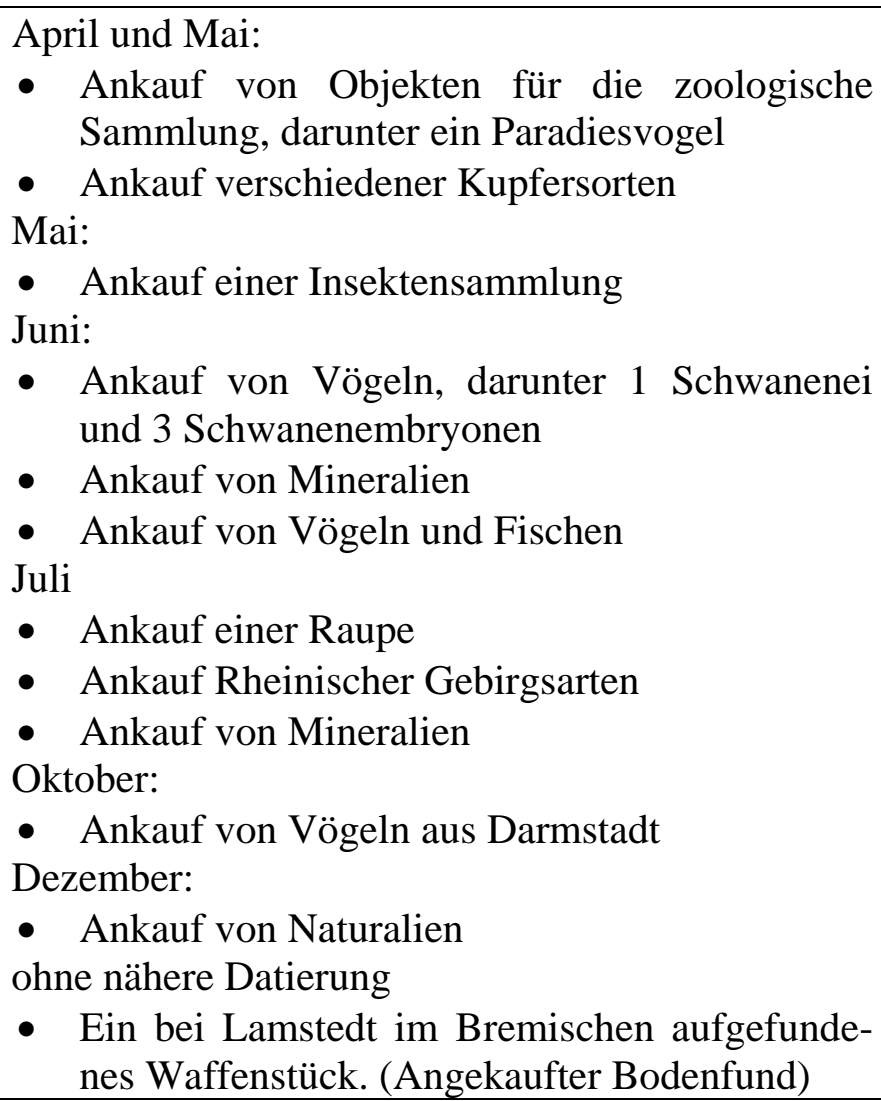 & $\begin{array}{l}\text { UAG Kur } 4 \text { V g 20, } \\
\text { 153, 155, 156, 168, } \\
\text { 186; UAG Kur } 4 \text { V } \\
\text { g } 42\end{array}$ \\
\hline 1840 & $\begin{array}{l}\text { Mai: } \\
\text { - Ankauf von Naturalien aus Würzburg u.a. eine } \\
\text { Antilope } \\
\text { April: } \\
\text { • Ankauf von Tierbälgen } \\
\text { Mai: } \\
\text { • Ankauf von Fischen } \\
\text { Juni: } \\
\text { - Ankauf eines Eisbärenfells aus dem Leipziger } \\
\quad \text { Museum }\end{array}$ & $\begin{array}{l}\text { UAG Kur } 4 \text { V g 20, } \\
186\end{array}$ \\
\hline
\end{tabular}




\begin{tabular}{|c|l|l|}
\hline Zeitpunkt & Art und Herkunft der Objekte & Belegstellen \\
\hline & $\begin{array}{l}\bullet \quad \text { Ankauf einer Insektensammlung } \\
\text { - Ankauf von Mineralien }\end{array}$ & \\
\hline $1840-1841$ & $\begin{array}{l}\text { Ankauf der naturhistorischen Sammlungen des } \\
\text { verstorbenen Obermedizinalrats Blumenbach. }\end{array}$ & UAG Kur 4 V g 46 \\
\hline
\end{tabular}

Neben diesen nachweisbar erfolgten Zugewinnen gab es eine Reihe von Sammlungen und Sammlungsgegenständen, deren Anschaffung erwogen, letztlich jedoch, meist aus Kostengründen, nicht umgesetzt wurde. Im Einzelnen handelt es sich um:

\begin{tabular}{|c|c|c|}
\hline \multicolumn{3}{|c|}{ Übersicht über angebotene / erwogene Sammlungsankäufe 1773-1840 } \\
\hline Zeitpunkt & Art und Herkunft der Objekte & Belegstellen \\
\hline 1790 & $\begin{array}{l}\text { Ankauf der Naturalien-Sammlung des Ingenieur- } \\
\text { Hauptmanns Müller. }\end{array}$ & UAG Kur 4 V g 12 \\
\hline 1803 & $\begin{array}{l}\text { Antrag der Witwe des Leibchirurgus Lampe wegen } \\
\text { Ankaufs des von ihrem Manne gesammelten Kabi- } \\
\text { netts. }\end{array}$ & UAG Kur 4 V g 16 \\
\hline 1817 & $\begin{array}{l}\text { Antrag des hiesigen Ordens-Genealogisten Schädt- } \\
\text { ler zum Ankauf eines prachtvollen Natur- und } \\
\text { Kunst-Kabinetts. }\end{array}$ & UAG Kur 4 V g 22 \\
\hline 1825 & $\begin{array}{l}\text { Anerbieten wegen Ankaufs der von dem Hofrat } \\
\text { Merrem zu Marburg hinterlassenen Sammlung von } \\
\text { ausgestopften Tieren. }\end{array}$ & UAG Kur \\
\hline 1829 & $\begin{array}{l}\text { Das zum Verkauf angebotene Naturalien- und } \\
\text { Kunst-Cabinet des weil. Konsistorialrats Canonicus } \\
\text { de la Tour zu Hildesheim. }\end{array}$ & UAG Kur 4 V g 33 \\
\hline $1834-1838$ & $\begin{array}{l}\text { Der in Antrag gebrachte Ankauf der Mineralien- } \\
\text { Sammlung des weil. Hofraths Bouterweck, incl. die } \\
\text { vom Amts-Assessor Römer in Hildesheim zum An- } \\
\text { kaufe dargebotene Sammlung von Versteinerungen. }\end{array}$ & UAG Kur 4 V g 35 \\
\hline 1832 & $\begin{array}{l}\text { Ankauf einer Sammlung Metallgegenstände von } \\
\text { dem Chaussee-Inspektor Horn. [Verhandlungsver- } \\
\text { lauf ist offen.] }\end{array}$ & UAG Kur 4 V g 34 \\
\hline 1840 & $\begin{array}{l}\text { Das zu Gölenkamp im Bentheimschen aufgefunde- } \\
\text { ne altertümliche Gefäß. }\end{array}$ & UAG Kur $4 \mathrm{~V}$ g 44 \\
\hline 1840 & $\begin{array}{l}\text { Die zum Kaufe angebotenen naturhistorischen } \\
\text { Sammlungen des Hofmedicus Dr. Zincken, genannt } \\
\text { Sommer, zu Braunschweig. }\end{array}$ & UAG Kur 4 V g 45 \\
\hline
\end{tabular}

Im Rahmen einer objektgeschichtlich ausgerichteten Studie müsste an dieser Stelle eine ausführliche Bewertung dieser Zugewinne und ein Diskussion folgen, in der z.B. auf die Motive der Gebenden und ihre Verbindung zur Georgia Augusta eingegangen wird. Dies soll, da in dieser Arbeit primär die Akteure von Interesse sind, im 
Folgenden nur kurz angerissen werden. Die Anzahl der im Laufe der Zeit hinzugewonnenen Objekte erscheint auf den ersten Blick recht groß. Dies relativiert sich jedoch stark, wenn man sich vor Augen führt, dass ein Zeitraum von 67 Jahren abgedeckt wird. Berücksichtigt werden muss aber ebenso, dass hier nur die in den Verwaltungsquellen belegbaren Zugewinne des Museums erfasst wurden. Um über die Qualität der Zugewinne hinsichtlich ihres wissenschaftlichen Gebrauchswertes zuverlässige Aussagen treffen zu können, wäre die Zurateziehung von Mineralogen, Zoologen und Ethnologen notwendig. Jedoch scheint es, als bliebe durch die Art der Schenkungen, zumal wenn es sich um Bodenfunde handelte, viel dem Zufall überlassen und als wäre ein kontinuierlicher Ausbau der Sammlungen unter forschungspraktischen Aspekten unterblieben. Auffallend ist, dass in den Akten fast ausschließlich Blumenbach als Impulsgeber auftritt, was sich dadurch erklären lässt, dass er (nach 1810) die oberste Instanz des Museums darstellte, und dass die Anträge von ihm formuliert oder zumindest unterzeichnet waren, was nicht mit der Idee für einen Ankauf gleichgesetzt werden kann. Als am schwächsten vertretene Kategorie in der Göttinger Sammlungspraxis erscheint der intermuseale Kauf- oder Tauschverkehr. Aus der Auflistung geht hervor, dass es sich bei den Sammlungszugewinnen vor allem um Schenkungen handelte. Dies mag der Tatsache Rechnung tragen, dass es, um Ankäufe tätigen zu können, bis 1837 notwendig war, für jede größere Erwerbung das Einverständnis der Landesregierung in Hannover einzuholen - erst 1837 erhielt das Museum einen eigenen Etat. ${ }^{270}$ Ein Großteil der Schenkungen fällt zudem in die Zeit kurz nach der Gründung des Museums, die Zeit seiner Etablierung. Gerade für das Jahr 1778 lassen sich viele kleine Geschenke nachweisen, die man als Besuchergaben, als eine Art Mitbringsel interpretieren könnte. Daneben heben sich die umfangreichen Geschenksendungen des Barons von Asch ab, der, der Göttinger Universität als Alumnus eng verbunden, auch nach diesem Zeitraum noch, vor allem der Bibliothek regelmäßig Geschenke zukommen ließ - und dafür von Heyne im Gegenzug mit „Literatur, die in St. Petersburg nicht aufzutreiben war“ versorgt wurde. ${ }^{271}$

${ }^{270}$ Vgl. in Kap. 5.1.1 den Abschnitt zu Arnold Adolph Berthold.

271 Helmut Rohlfing, Eine neue russische Bibliothek in Göttingen. Georg Thomas von Asch als Förderer der Georgia Augusta, in: 300 Jahre St. Petersburg: Russland und die „Göttingische Seele“; Ausstellung in der Paulinerkirche Göttingen unter der Schirmherrschaft von Bundespräsident Johannes Rau und dem Präsidenten der Russischen Föderation Wladimir Putin, Göttingen 2003, 287-302, 290. 
Welche weiteren Motive den Gaben zugrunde lagen, lässt sich nur schwerlich verallgemeinern. Bezüglich der Nachlässe könnte man ein mangelndes Interesse oder mangelnde finanzielle Ressourcen zur Weiterführung dieser Sammlungen vermuten. Einen Eindruck von der Haltung der (zukünftigen) Erben der Besitzer solcher Kabinette liefert z.B. das Ankaufsangebot des Sohnes des Hofmedikus' Zincken aus Braunschweig. Dort heißt es, dass die Sammlung dem Museum angeboten werde

damit dadurch eines theils das Loos des Vereinzelns seine Sammlung nicht treffe und als anderstheils das sonst so berühmte Museum zu Göttingen gerade in Beziehung auf die Gegenstände, in welchen meines Vaters Sammlung excellirt, höchst unvollkommen ist. ${ }^{272}$

Aus der dargestellten Sammlungs-Praxis lässt sich die These ableiten, dass Personen, die mit der Universität oder einzelnen ihrer Angehöriger in Verbindung standen, ein weit höheres Spendeninteresse hatten als Personen, die der Institution Museum ohne persönlichen Bezug gegenüberstanden. Darüber hinaus setzt die Bereitschaft zur Gabe an das Museum eine bestimmte Geisteshaltung voraus: Akzeptanz der (Unter-)Institution Museum, Wertschätzung der Universität und/oder ihrer Vertreter und der Wille, die Wissenschaften zu fördern - oder sich durch umfangreiche Spendentätigkeit eine Art Memorialort zu schaffen. Hier tritt wiederum die Rolle der Alumni und auch und vor allem die Größe der Reputation der Universität in den Blick: In der Zeit des größten Spendenaufkommens genoss die Georgia Augusta den Ruf, die führende Universität des Landes zu sein.

\subsection{Sammlungsverluste}

Der verhältnismäßig großen Zahl an Sammlungszugängen für das Academische Museum steht ein einzelner, allerdings recht spektakulärer Sammlungsverlust gegenüber: Der Diebstahl einer großen Silberstufe in der Nacht vom 15. auf den 16. Januar 1783. Die Stufe war im Zuge der Überführung der Schlüterschen Sammlung aus Hannover fünf Jahre zuvor ans Museum gelangt, enthielt „einen Centner am Gewicht““273 und besaß einen Wert von 1600 Rthlr. ${ }^{274}$ Es gehört zur Ironie dieser Geschichte, dass Lichtenberg im Vorfeld berichtete, dass man dieses große Stück ge-

\footnotetext{
272 UAG Kur 4 V g 45, Sommer jr. an das Ministerium in Hannover vom 29. November 1840, 1r.

273 Vom Academischen Museum herausgegebenes Flugblatt vom 16. Januar 1783, in dem zur Beihilfe der Aufklärung des Diebstahls aufgerufen wird. Zitiert nach HANS-HEINRICH HIMME, Stich-haltige Beiträge zur Geschichte der Georgia Augusta in Göttingen. 220 Stiche aus den ersten 150 Jahren der Göttinger Universität; zusammengetragen und mit Texten versehen anläßlich ihres 250jährigen Jubiläums, Göttingen - Zürich 1987, 80. Original in UAG Kur 3 k 17.

274 Vgl. Lichtenberg an Hollenberg vom 3. April 1777, zitiert nach HiMME, Stichhaltige Beiträge, 80.
} 
diegenen Silbers bei Führungen im Scherz „demjenigen umsonst anbot, der es wegtragen könte.“ ${ }^{275}$ Der Diebstahl dieser Stufe wurde nicht aufgeklärt und die Stufe auch nicht wieder aufgefunden. Allerdings gab es recht eindeutige Hinweise darauf, dass sie wohl zertrümmert und dann in einzelnen Stücken weiterverkauft oder eingeschmolzen wurde. ${ }^{276}$ Vor allem Autoren späterer Epochen fanden augenscheinlich Freude daran, Geschichte in Geschichtchen zu verpacken und von den damaligen Vorkommnissen zu berichten. So griff z.B. Otto Denecke 1938 in einem Band seiner Göttinger Nebenstunden die Begebenheiten unter dem Titel „Göttingen heißt Tuliphurdum?“ in sein Repertoire auf. Hans Heinrich Himme nahm das Ganze gar unter dem Titel „Ein Kriminalstück im Jahre 1783“ in seine Stich-haltige[n] Beiträge zur Geschichte der Georgia Augusta in Göttingen auf. Silke Wagener hingegen gewann durch diesen Vorfall Aufschlüsse über den Aufgabenbereich von Universitätsunterbedienten, namentlich Museumswärtern. ${ }^{277}$

Außer Schäden durch Mottenfraß lassen sich im Untersuchungszeitraum ansonsten keine dauerhaften Sammlungsverluste aufzeigen. ${ }^{278}$ Allerdings ist in diesem $\mathrm{Zu}$ sammenhang auf eine Umverteilung von Sammlungsobjekten innerhalb Göttingens hinzuweisen. So waren beim Umzug in die ehemaligen Professorenhäuser die im Catalogus Musei Academici noch aufgeführten Herbarien vom Museum an den botanischen Garten abgetreten worden. ${ }^{279}$

\subsection{Eine Ordnung der Dinge und ein neuer Standort}

Informationen über die Aufstellung der Bestände im Akademischen Museum oder auch die Aufteilung und Nutzung der Räume sind rar gesät. Primärquellen, wie schematische Aufstellungsskizzen oder Stellpläne, scheinen nicht erhalten zu sein. Die einzige auffindbare zeitgenössische Quelle, die darüber berichtet, ist die zwischen 1765 und 1838 in vier stets aktualisierten und ergänzten Bänden erschienene

\footnotetext{
275 Lichtenberg an Hollenberg vom 3. April 1777, zitiert nach HiMME, Stich-haltige Beiträge, 80.

276 Vgl. Blumenbach an Heyne vom 30. Jan. 1783. Abgedruckt in HiMME, Stich-haltige Beiträge, 82.

277 Vgl. Silke Wagener, Pedelle, Mägde und Lakaien. Das Dienstpersonal an der Georg-AugustUniversität Göttingen 1737-1866, Göttingen 1996, 353; zum Aufgabenbereich der Museumswärter vgl. Kap. 5.1.2 der vorliegenden Arbeit.

278 Die Rückgabe von Sammlungsobjekten an das Museum in Kassel, die während der napoleonischen Ära nach Göttingen gelangt waren werden ebenso wenig als dauerhafte Sammlungsverluste betrachtet wie ihre Anlieferung als dauerhafter Gewinn.

279 Vgl. SAALFELD, Gelehrten-Geschichte (3), 419.
} 
Universitätsgeschichte Pütters. ${ }^{280}$ Diese liefert sowohl für die Zeit, in der das Museum bei der Universitätsbibliothek untergebracht war, als auch für die Zeit, in der es seine eigenen Räumlichkeiten in den ehemaligen Professorenwohnhäusern am Papendiek erhielt, einen Eindruck von der Gliederung des Museums und der Aufstellung der Objekte. ${ }^{281}$ Für die Zeit, in der das Museum im ehemaligen Medizinischen Auditorium des Kollegienhauses untergebracht war erfährt man beispielsweise, dass die „Münzsammlung [...] in zwey Schränken verwahret““282 wurde, und dass

\begin{abstract}
„[d]as ganze Museum [...] in vier Hauptabschnitte eingetheilt [ist], wovon der I. bloß das begreift, was zur Naturgeschichte des Menschengeschlechts im weitesten Umfange gehört; folglich auch alles, was die Lebensart, eigenthümliche Sitten u. fremder Völkerschaften betrifft: der II. das übrige Thierreich: der III. die Gewächse: und der IV. die Mineralien. Das Ganze ist nach der dritten Ausgabe des Blumenbachischen Handbuchs geordnet; die Säugethiere nehmlich nach des Verfassers eignem Systeme: die übrigen Thiereclassen mit nöthigen Abänderungen nach dem Linnéschen: ${ }^{283}$ so das Mineralreich mit eben dieser Einschränkung nach Kirwan's System: ${ }^{284}$ und die Petrefacten nach der unten anzuführenden Rücksicht.“ 285
\end{abstract}

Hier wird deutlich, dass bereits bei der Einrichtung des Museums eine Aufteilung in vier inhaltlich (jedoch nicht verwaltungsmäßig) getrennte Abteilungen vorgenommen worden ist. Zum anderen erfahren wir, dass das Museum - zu dieser Zeit - nach modernsten wissenschaftlichen Erkenntnissen strukturiert war. „Schon nach wenigen Jahren - aktenmäßig jedoch im Zeitpunkt nicht genau feststellbar - kristallisiert sich aus der ersten Abteilung, [...], als selbständiger Teil die ,Ethnographische Sammlung’ heraus.“ ${ }^{286}$ Das starke Anwachsen des Bibliotheksbestandes und auch das stetige Wachstum der Museumssammlungen führten binnen kürzester Zeit zu Raumnot im Kollegienhaus. Um die Stellflächen zu erweitern, wurden die der Bibliothek gegenüberstehenden ehemaligen Professorenhäuser längs des Papendiek für die Universität angekauft (Abb. 4).

280 Johann Stephan Pütters Versuch einer academischen Gelehrten-Geschichte von der GeorgAugustus-Universität zu Göttingen, 4 Teile, 1765-1838, Teil 3 von Friedrich Saalfeld und Teil 4 von Georg Heinrich Oesterley fortgesetzt.

281 Während die räumliche Ordnung des Museums nur über Pütter nachzuvollziehen ist, finden sich vergleichsweise viele Beschreibungen der im Museum aufgestellten Objekte.

282 PÜTTER, Gelehrten-Geschichte (2), 232.

283 Der schwedische Naturforscher Carl von Linné (1707-1708) entwickelte ein im 18. Jahrhundert breit rezipiertes, inzwischen aber als überholt geltendes Klassifikationssystem für Pflanzen. Sein großes Verdienst ist die Schaffung einer binären Nomenklatur, mit der er für jedes Lebewesen lateinische Gattungs- und Artnamen als international verständliche Bezeichnungen einführte.

284 Richard Kirwan (1733-1812) war ein irischer Chemiker und Mineraloge, der sich zudem mit Meteorologie und Geologie befasste. Sein 1784 erschienenes Werk Elements of Mineralogy gilt als erstes (englischsprachiges) mineralogisches Referenz(hand)buch überhaupt.

285 PÜTTER, Gelehrten-Geschichte (2), 233.

286 PLISCHKE, Ethnographische Sammlung, 13. 


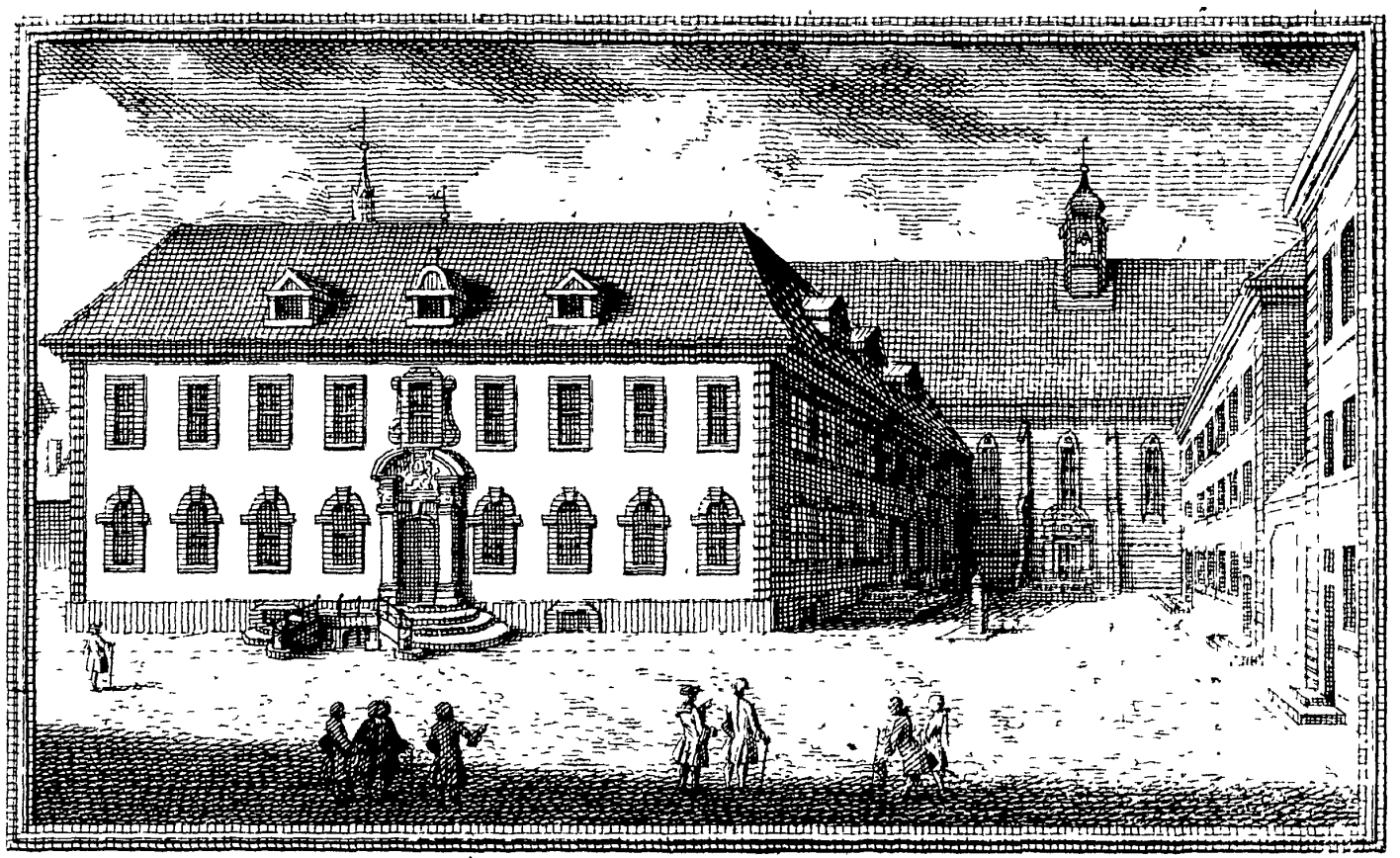

Abbildung 4: Blick auf das Universitätsensemble (von Norden): links das Kollegienhaus, hinten die Paulinerkirche, rechts die Professorenhäuser (1747).

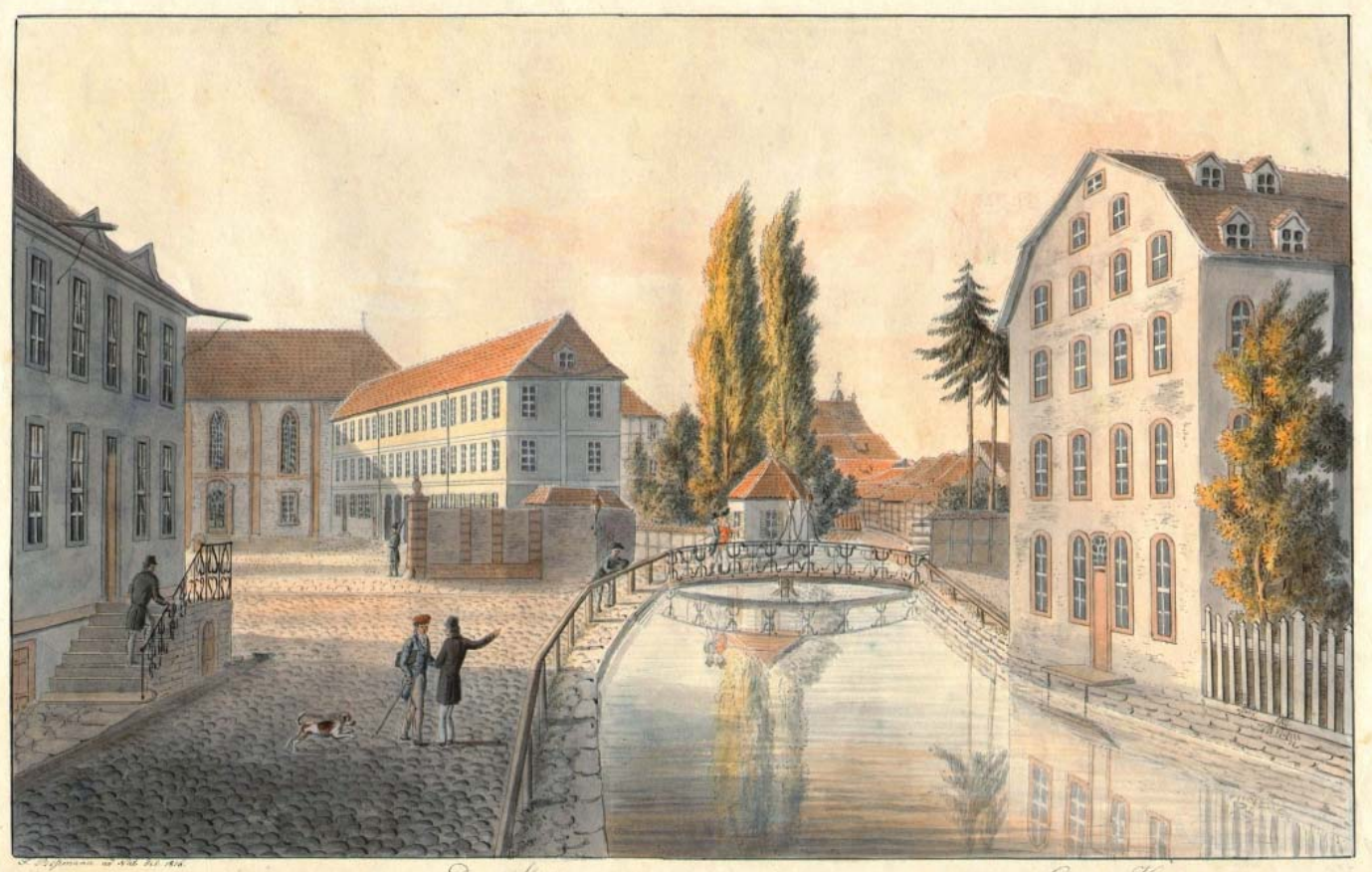

Abbildung 5: Blick auf die ehemaligen Professorenhäuser (von Norden), die von 1793 an das Academische Museum und weitere Universitätssammlungen beherbergten (1816). 
1793 zogen die Sammlungen des Academischen Museums in diese Räumlichkeiten um. ${ }^{287}$ Leider sind weder die betreffenden Gebäude noch Pläne von ihnen erhalten. ${ }^{288}$ Abbildung 5 vermittelt jedoch einen Blick von der Lage des Museums im Universitätskomplex und von der Größe des Gebäudes. ${ }^{289}$ Darin wurden nicht nur die Sammlungen des Academischen Museums, sondern auch die Modellkammer, die Gemäldesammlung und das physikalische Kabinett untergebracht. Wo genau die Sammlungen Aufstellung fanden, z.B. in welchem Stockwerk, ist nicht überliefert. In den Verwaltungsakten finden sich keine Angaben über die Aufteilung des Gebäudes für die einzelnen Sammlungen oder gar Stellpläne. Mittels der verschiedenen Ausgaben der Gelehrtengeschichte Pütters, lässt sich jedoch zumindest die grobe Strukturierung der Räumlichkeiten nachvollziehen. So schrieb Saalfeld 1820:

„Jetzt ist das Museum in 14 Zimmer $^{290}$ vertheilt; sieben für die zoologische Abtheilung; fünfe für die mineralogische; und zwei für die ethnographische, der auch das was von Kunstsachen des Alterthums dem Museum zu Theil wird, beigefügt ist.“ “291

Die weiteren Ausführungen Saalfelds befassen sich vor allem mit einzelnen Objekten und der Auseinandersetzung was von wem geschenkt worden ist, geben jedoch keinen weiteren Aufschluss zur Positionierung der Objekte. Fast zwanzig Jahre später heißt es im vierten Band der Pütterschen Gelehrtengeschichte „Die Einrichtung des Museums ist im Wesentlichen dieselbe geblieben“²92. Erneut galt die Aufmerksamkeit vor allem neuen Sammlungszugewinnen. Über die Veränderungen, die nach Blumenbachs Tod im Museums vor sich gingen und die letztlich v.a. auf den Ankauf der umfangreichen Privatsammlung Blumenbachs zurückzuführen sind, lässt sich indirekt erschließen, dass sich die zoologische Sammlung vor 1840 im mittleren Stockwerk des Gebäudes befand.

287 In Pütters Gelehrten-Geschichte werden über den Zeitpunkt des Umzugs unterschiedliche Angaben gemacht. Band 3 setzt als Umzugsjahr 1793 fest, Band 4 1795. Vgl. SAALFELD, GelehrtenGeschichte (3), 419 und OEsTERLEY, Johann Stephan Pütters Versuch einer academischen Gelehrten-Geschichte von der Georg-Augustus-Universität zu Göttingen Theil 4: Von 1820 bis zur ersten Säcularfeier der Universität im Jahre 1837. Mit 7 Kupfern, Göttingen 1838, 90f.

288 Sie wurden 1878 wegen Baufälligkeit abgerissen und durch einen Erweiterungsbau für die Universitätsbibliothek ersetzt. Vgl. EHLERS, Göttinger Zoologen, Berlin 1901, 81.

289 Eine kurze bauliche Beschreibung des Gebäudes findet sich in UAG Kur 3198. Diese stammt allerdings erst aus dem Jahr 1867.

290 Für das Jahr 1815 lässt sich der Umfang des Museums auf 15 Räume bestimmen. Dies geht aus einem auf den 25. September 1815 datierten Schreiben Blumenbachs an das KabinettsMinisterium in Hannover hervor. Vgl. UAG Kur 4 V g 5, 36r-37r, 36r. Für die Zeit nach 1840 nimmt Ehlers eine Lokalisierung der Sammlungen vor. Vgl. DERS., Zoologen, bes. 44f.

291 SAALFELD, Gelehrten-Geschichte (3), 419.

292 OESTERLEY, Gelehrten-Geschichte (4), 90. 


\section{$4 \quad$ Im neuen Jahrhundert}

Mit Beginn des 19. Jahrhunderts kann die Etablierungsphase des Museums als abgeschlossen gelten. Mehr noch: man muss sich fragen, ob es nicht in gewisser Weise schon seinen Zenit überschritten hatte: die Anzahl der beim Museum eintreffenden Zugewinne war rückläufig, geplante Katalogvorhaben kamen nicht zur Realisierung, eine Aufstellung oder gar Umsetzung neuer Ordnungssysteme ist nicht überliefert. Man ist versucht, von einer ruhigen Zeit zu sprechen, wären da nicht personelle Veränderungen, die Wirren der napoleonischen Zeit, und einige grundlegende Veränderungen in den 1830er Jahren.

\subsection{Personelle Veränderungen}

Der nachstehende Abriss stellt gleichermaßen einen Vorgriff und eine Ergänzung zu Kapitel 5.1.1 dar. Hier wie dort kommen die Hauptpersonen des Academischen Museums in den Blick. Während in Kap. 5.1.1 auf die jeweiligen Biographien eingegangen wird, stehen die Aufseher hier als Funktionsträger des Museums im Mittelpunkt. Veränderungen der Stellung bzw. der ausübenden Person stehen für Indikatoren von Wandlungsprozessen.

Nachdem das Museum zwischen 1792 und 1795 mit Friedrich Meyer kurzzeitig einen zweiten Unteraufseher besessen hatte, lag die Leitung zur Jahrhundertwende wieder in den Händen Blumenbachs und Heynes. Hier trat Blumenbach, auf der Höhe seines wissenschaftlichen Schaffens jedoch zunehmend in den Vordergrund. 1810 übernahm er die Position des Oberaufsehers, die er bis zu seinem Tod 1840 innehaben sollte. Nach dem Tod Heynes führte Blumenbach die Geschäfte des Museums zunächst allein weiter. 1812 wurde ihm der junge Privatdozent Johann Friedrich Osiander als Hilfskraft beim Ordnen der Sammlungen zur Seite gestellt. 1815 besserte sich die Ausstattung des Museums auf Dauer, als Osiander zum Unteraufseher über die zoologische und ethnographische Sammlung des Museums bestimmt und zur gleichen Zeit Johann Friedrich Ludwig Hausmann als Unteraufseher der mineralogischen Sammlung berufen wurde. Diese personelle Konstellation blieb bis 1827 bestehen. In jenem Jahr schied Osiander - vorübergehend - aus der Aufsicht über das Museum aus. Diese Lücke wurde durch die Einstellung August Murrays geschlossen, 
der jedoch nur eine Stelle als Assistent, und nicht als Unteraufseher erhielt. Murrays Stelle wurde nach Jahresfrist durch Ernst Friedrich Gustav Herbst neu besetzt, als Murray sich entschloss, Göttingen zu verlassen. Herbst blieb bis zur Rückkehr Osianders in seine alte Position als Unteraufseher im Jahr 1833. Frischen Wind erhielt das Museum als 1836 Arnold Adolph Berthold die Mitaufsicht über die Zoologische Sammlung übertragen wurde. Die - zumindest nach außen hin einschneidendste personelle Veränderung bildete jedoch der Tod Blumenbachs im Jahr 1840. Seine Nachfolge trat der Zoologe Rudolf Wagner an.

\subsection{In der napoleonischen Zeit}

In der Zeit, in der Göttingen dem Königreich Westfalen eingegliedert war (18071813) kam in kurzer Zeit viel Bewegung in die Sammlung ${ }^{293}$ - die jedoch nur wenige nachhaltige Auswirkungen hatte, weshalb an dieser Stelle auch nur kurz darauf eingegangen werden soll. Die angesprochene Dynamik ist vor allem auf zwei Aspekte zurückzuführen: Zum einen Sammlungszugewinne und zum anderen die Erstellung eines Verzeichnisses der - inzwischen durch den Forster-Nachlass ergänzten Cook-Sammlung. Offensichtlich begegnete die „General-Direction des öffentlichen Unterrichts des Königsreichs Westphalen“ dem Academischen Museum mit wohlwollendem Interesse, was wohl hauptsächlich auf Blumenbachs Renomee zurückzuführen ist. $^{294}$

Sowohl für die französische als auch für die Göttinger Seite lassen sich Bestrebungen nachweisen, Objekte aus dem Kasseler Carolinum nach Göttingen zu überführen. ${ }^{295}$ Bei dem stattfindenden Transfer spielte Gravenhorst ${ }^{296}$ eine wichtige Rolle. Augenscheinlich war er dafür zuständig, die Auswahl der für Göttingen interessanten Gegenstände aus den Beständen in Kassel zu treffen. ${ }^{297}$ Auf die betreffenden Objekte

293 Aus diesem vergleichsweise kurzen Zeitraum ist ein 93-seitiges Aktenkonvolut überliefert. Vgl. UAG Kur 3 V g 19.

294 So ist in den Schriftwechseln immer wieder von „Le celèbre Blumenbach“ die Rede. Vgl. z.B. das Schreiben J.O. Mullers an den Minister des Inneren vom 10. Mai 1809 in UAG Kur 4 V g 19, 14r.

295 Vgl. UAG Kur 4 V g 19 bes. 14-27.

${ }^{296}$ Zu Gravenhorst vgl. Kap. 5.1.1.

297 Dafür spricht zudem das von Gravenhorst abgefasste „Verzeichniß derjenigen naturhistorischen Gegenstände welche von dem Casselschen an das Göttingische Museum abgegeben, und von mir, unterschriebenen zweiten Aufseher des Museums zu Göttingen in Empfang genommen sind.“ vom 2. April 1809. AVkG, Akten 1: Altakten (Lose-Blatt-Sammlung), Alte Dokumente 19. Jh; vgl. ferner den Schriftverkehr in UAG Kur 4 V g 19. 
soll hier nicht näher eingegangen werden, denn bereits im Dezember 1814 wurde nach Aufforderung aus Kassel alles in die Wege geleitet, um diese Objekte zurückzuführen. ${ }^{298}$ Neben den Objekten aus Kassel gelangten weitere Gegenstände, vor allem aus dem in Auflösung begriffenen Georgianum in Hannover, nach Göttingen. ${ }^{299}$ Für die weitere Akquise von Sammlungsstücken verstand es Blumenbach geschickt die politische Situation zu nutzen. Als es darum ging, eines in der Nähe von Helmstedt aufgefundenen Meteoriten für das Museum habhaft zu werden, führt er gegenüber der französischen Seite an, dass dieser sich „bei dem dasigen Friedensrichter befinde, welcher willens seÿ, denselben nach Berlin zu schicken“300 - damit wäre er den Preußen in die Hände gefallen, was die Franzosen nicht gutheißen konnten.

Der zweite, für die napoleonische Ära besonders interessante Punkt, ist die von französischer Seite ebenso höflich wie bestimmt angeforderte Auflistung des Museumsinhalts, insbesondere der Cook-Sammlung. Für den dadurch erhöhten Arbeitsaufwand wurde Blumenbach ein Gehilfe zugestanden, der letztlich dauerhaft am Museum bleiben sollte: Johann Friedrich Osiander kam 1812 ans Museum und verfasste in der Folgezeit das geforderte Verzeichnis über die (Kunstsachen) Handarbeiten und Naturalien aus den Südsee Inseln.

\subsection{Ruhige Zeiten oder Stagnation?}

Bis auf die oben angeführten, in Kapitel 5.1.1 näher erläuterten personellen Veränderungen und die bereits in Kapitel 3.1 behandelten Sammlungszugewinne lassen sich für den Zeitraum von der Wiedereingliederung Göttingens ins Königreich Hannover bis zur Säkularfeier der Universität keine bedeutenden Veränderungen nachweisen. Hier kann man tatsächlich von ruhigen Zeiten sprechen. Bezieht man die in Kap. 3.1 erwähnte rückläufige Tendenz an Sammlungszugewinnen sowie das steigende Alter Blumenbachs mit ein, scheint auch die Zuschreibung Stagnation gerechtfertigt. 1837 trat jedoch eine Wende ein. Zum einen erfolgte auf Initiative Bertholds die Zuweisung eines - wenn auch zunächst nur geringen - Jahresetats an das Museum. Zum anderen kam es zu einer Kodifizierung und Ausweitung bestehender Praktiken

\footnotetext{
298 Vgl. UAG Kur 4 V g 21.

299 Vgl. UAG Kur 4 V g 19, 27-30.

300 UAG Kur 4 V g 19, Schreiben Blumenbachs an den Generalstudiendirektor (ohne Datierung); ferner zu diesem Vorgang, UAG Kur 4 V g 19, 41-47.
} 
bzw. Rechte. Mit Schreiben vom 5. April 1838 gestattete die Regierung in Hannover der Direktion des Academischen Museums, selbiges nach Ausfertigung einer den Zutritt regelnden Anweisung, einmal wöchentlich unentgeltlich für Studenten zu öffnen. ${ }^{301}$ Zudem wurde das Abhalten von Demonstrationsstunden innerhalb der verschiedenen Abteilungen festgelegt. Dies ist insofern neu, als bis zu diesem Zeitpunkt keine verbindlich festgelegte Besucherregelung für das Museum existiert. Jedoch scheint mit Ausnahme der regelmäßigen Öffnungszeit außerhalb des akademischen Unterrichts keine tief greifende Veränderung in den Nutzungsmöglichkeiten erfolgt zu sein.

\subsection{Das Ende einer Ära und ein Neubeginn - Ausblick auf die Entwicklung nach dem Tod Blumenbachs}

Bereits vor Blumenbachs Tod (1840) hatten die wesentlichen Amtsgeschäfte des Museums in den Händen der Unteraufseher der einzelnen Abteilungen gelegen. Darauf weisen zahlreiche Rechnungen vom Ende der 1830er Jahre hin, die von Hausmann, Osiander oder Berthold oder auch allen dreien unterzeichnet sind. Trotzdem bedeutete der Tod Blumenbachs, wie an anderer Stelle bemerkt, eine entscheidende Zäsur für das Academische Museum, nicht jedoch sein Ende. Vielmehr wurde eine neue Entwicklungsphase eingeleitet, ${ }^{302}$ die damit begann, dass das Museum einer Verwaltungsreform unterzogen wurde.

Nach etwas mehr als einem halben Jahr Vakanz war am 10. September 1840 Rudolf Wagner (1805-1864) in die Direktion des Academischen Museums berufen worden. Er fand, wie Plischke zu berichten weiß, „unhaltbare[n] Zustände“ vor, die ihn dazu veranlassten, bereits am 17. Oktober desselben Jahres einen längeren Bericht an die Regierung in Hannover abzufassen. ${ }^{303}$ Mit dem Argument, dass „die einzelnen Gebiete, die in den Sammlungen des Academischen Museums vereint waren, jetzt zu groß geworden wären, um noch von einem übersehen werden zu können“, ${ }^{304}$ trat er

\footnotetext{
301 Vgl. UAG Kur 4 V g 39.

302 Dokumentationen darüber finden sich vor allem bei Ehlers, Zoologen, 55-82, mit Schwerpunkt auf der Zoologie, jedoch auch für die anderen Sammlungsbereiche informativ; und bei PLISCHKE, Ethnographische Sammlung, 39-46, mit so starker Schwerpunktsetzung auf der völkerkundlichen Sammlung, dass darüber die anderen Sammlungen vernachlässigt werden.

303 PLISCHKE, Ethnographische Sammlung, 39.

304 PlisCHKE, Ethnographische Sammlung, 40.
} 
für eine größere Eigenständigkeit der einzelnen Abteilungen ein, die auch gewährt wurde. Sukzessive entstanden vier verwaltungs- und weitgehend auch etatmäßig selbstständige Abteilungen: „die mineralogisch-geologische unter Hausmann; die zoologische unter Berthold; die anthropologisch-zootomische unter Wagner; die ethnographische unter Osiander [...].““305 Diese Struktur wurde, trotz mehrmaliger Personalwechsel im Wesentlichen bis ins Jahr 1878 beibehalten. Dabei gewann die zoologisch-zootomische Sammlung im Laufe der Zeit zunehmend an Bedeutung.

Der ständig wachsende Umfang der Sammlungen auf der einen Seite und die Baufälligkeit des Hauses, in dem sie untergebracht waren auf der anderen führte dazu, dass 1876 mit dem Bau eines Naturhistorischen Museums begonnen wurde. 1878 war der Umzug abgeschlossen. Im unmittelbaren Anschluss daran wurde das alte Museumsgebäude abgerissen. ${ }^{306}$ Damit verschwanden die letzten sichtbaren Zeichen der schon seit langem nur noch nominell existierenden Institution Academisches Museum.

Insgesamt gesehen zeichnet sich die Entwicklung des Academischen Museums im 19. Jahrhundert durch eine gewisse Behäbigkeit aus. Diese erscheint insofern logisch, als sich das Museum nicht mehr in einer Etablierungsphase, sondern in einer Konsolidierungsphase befand, die durch die Napoleonische Ära empfindlich gestört wurde. Nachfolgend verlief die Entwicklung des Museums stagnierend, was sich erst gegen Ende des Untersuchungszeitraumes hin wieder änderte.

305 PLISCHKE, Ethnographische Sammlung, 39.

306 EHLERS, Zoologen, 81. 


\section{$5 \quad$ Akteure und Nutzung}

\subsection{Das Personal}

Der Betrieb eines Museums ist ein komplexes Unterfangen, welches die möglichst reibungslose Interaktion ganz verschiedener Personenkreise erfordert. Diese ergeben sich aus ihren unterschiedlichen Aufgaben im sowie ihren Interessen am Museum und seinen Exponaten. Auf der einen Seite steht das Personal, das im Falle des Academischen Museums Göttingen nicht nur für allgemeine Verwaltungsaufgaben wie z.B. das Abwickeln von Jahresrechnungsabschlüssen zuständig war, sondern vielmehr aktiv an der Gestaltung und Entwicklung des Museums teil hatte. Ihm oblag es, der bereits bestehenden, sich aber immer weiter entwickelnden Sammlung einen institutionellen Rahmen zu verschaffen, die Sammeltätigkeit nicht nur zu überwachen, sondern auch selbst akquisitorisch tätig zu werden, den Objekten eine Ordnung zu geben und nicht zuletzt für ihren ordnungsgemäßen Zustand und ihre Erhaltung Sorge zu tragen. Auf der anderen Seite - gelegentlich kommt es auch zu Überschneidungen - stehen die Besucher der Sammlungen. Sie benutzten die Sammlungsobjekte entweder für eigene wissenschaftliche Forschungen oder auch nur zur Befriedigung eines privaten Interesses, dienten dem Museum damit jedoch gleichzeitig, indem sie ihm durch ihre Arbeit oder auch nur ihre Neugier zu einer größeren Öffentlichkeit und einem gewissen Ansehen verhalfen. Eben dieses konnte dazu führen, dass sich Sponsoren -sowohl Privatpersonen als auch Institutionen - bereit fanden, v.a. dingliche Gaben zur Erweiterung der Sammlungen beizutragen. ${ }^{307}$ Eine wichtige Rolle spielten auch die Zulieferer. Zwar hängt deren Wirken nicht unmittelbar mit dem Museum zusammen, sie trugen aber gleichwohl wesentlich zur Erhaltung oder Präsentation der Exponate bei. ${ }^{308} \mathrm{Zu}$ diesem Personenkreis sind auch die Naturalienhändler zu rechnen. ${ }^{309}$

${ }^{307}$ Hier sind für das Academische Museum z. B. Baron von Asch, verschiedene Nachlassgeber oder auch die Societät der Wissenschaften zu nennen. Theoretisch ließe sich auch an finanzielle Unterstützung denken, dafür konnte jedoch kein einziger Fall nachgewiesen werden.

${ }^{308} \mathrm{Zu}$ denken ist hier an verschiedene Handwerker, die insbesondere Tischler- und Glaserarbeiten für Vitrinen ausführten, sowie an den Apotheker, der in regelmäßigen Abständen Spiritus für das Befüllen von Präparaten lieferte.

${ }^{309}$ In den Quellen erscheint diese Gruppe vor allem in Rechnungen, ist ansonsten aber nur schwer fassbar. 
In den folgenden Kapiteln wird näher auf die Mitglieder der beiden zuerst genannten Gruppen eingegangen. Dabei ist das Personal noch am besten erschließbar: Zum einen ist seine Zahl überschaubar und zum anderen tritt es in der Regel in den (Verwaltungs-)Akten in Erscheinung. Leider geht daraus nicht hervor, inwiefern Angehörige des Personals, die als einzige quasi uneingeschränkten Zugang zum Museum hatten, die Sammlung für eigene, z.B. wissenschaftliche, Zwecke nutzten. D.h., es ist weder etwas über die Frequenz ihrer Besuche noch über die Verweildauer oder die von ihnen ausgeführten Tätigkeiten zu erfahren - es sei denn, sie legten bewusst Zeugnis davon ab. Da die Verwaltungsquellen nur Schlaglichter auf ihren Aufgabenbereich werfen, wird hier auf Kurzbiographien des wissenschaftlich-administrativen Personals und der Aufseher zurückgegriffen. Damit soll zweierlei erreicht werden: zum einen lässt sich so herausarbeiten, welchen personellen Veränderungen das Museum unterworfen war, zum anderen erhalten die beteiligten Personen ein ,Leben außerhalb des Museums’ und können damit wissenschaftlich verortet werden.

Anschließend stehen die Besucher im Mittelpunkt, die zunächst eine amorphe Masse zu sein scheinen. Glücklicherweise existiert - wenn auch nur für einen begrenzten Zeitraum - ein Besucherbuch, das die einzelnen Besucher verzeichnet, wodurch sich die Frequentierung des Museums nachzeichnen lässt. Mitunter ist es zudem möglich, anhand dieser Daten weiterführende Quellen zu einzelnen Personen heranzuziehen. Hier sind vor allem Selbstzeugnisse der Besucher wie Tagebücher und Briefe hilfreich. ${ }^{310}$ Die Zutritts- und Nutzungsberechtigungen der Besucher waren in der Regel stark eingeschränkt und reglementiert; man erfährt in erster Linie, was sie nicht durften, und kann so Rückschlüsse darauf ziehen, was ihnen zu tun übrig blieb. All dem widmen sich die Kapitel 5.2 und 5.3.

\subsubsection{Die Aufseher der Sammlung}

Bei den Aufsehern der Sammlung handelte es sich um die eigentlichen Entscheidungsträger des Museums. Sie waren der Regierung direkt unterstellt und in finanziellen Dingen auf diese angewiesen. Alle Entscheidungen, die die Sammlung direkt betrafen, wie Aufstellung, Planung und Akquise von neuen Objekten oblag ihrer

310 Darüber hinaus könnte versucht werden, einen weiteren Teil der Besucher über das Umfeld, die personellen Netzwerke des Personals zu erschließen. Allerdings ist der hierbei zu erwartende Erkenntnisgewinn mit einem unverhältnismäßig hohen Aufwand verbunden. 
Verantwortung. Als Hauptakteur kann zweifelsohne Johann Friedrich Blumenbach gelten. Er hat das Academische Museum über 67 Jahre hinweg begleitet und geleitet, es gleichermaßen gefördert, wie letztlich auch in seiner Entwicklung behindert. Für die Frühzeit des Museums sind allerdings auch andere Charaktere von Bedeutung: Christian Wilhelm Büttner, der (Vor-)Besitzer der Naturaliensammlung, die den Grundstock des Academischen Museums bilden sollte, und Christian Gottlob Heyne, ihr - im doppelten Sinne - erster Aufseher. Nach und nach betraten weitere Akteure die Bühne. Sie werden im Folgenden in chronologischer Reihenfolge vorgestellt.

\title{
Christian Wilhelm Büttner
}

Am Anfang der Geschichte des Academischen Museums steht Christian Wilhelm Büttner, der auf der Grundlage der Sammlungen seines Vaters und Großvaters ein umfangreiches Naturalienkabinett aufbaute, ${ }^{311}$ das er 1773 der Universität gegen eine Leibrente $^{312}$ überließ. Wer aber war Büttner? Büttner wurde am 27. Februar 1716 als Apothekerssohn in Wolfenbüttel geboren. Er

\begin{abstract}
hatte in Leipzig studirt und sich, dem Berufe des Vaters folgend, zum Apotheker ausgebildet. Von Haus aus begütert, ging er auf Reisen, nach Norwegen und Lappland, Land, Leute und Sprache kennen zu lernen, von da nach Edinburg, wo er gälisch lernte, trieb dann in Oxford bei dem giessener Dillenius Botanik, und zog von da nach Leyden, um unter Boerhave zu studiren; hier wohnte er ein halbes Jahr zusammen mit Linné auf demselben Zimmer. ${ }^{313}$
\end{abstract}

Anschließend hatte er sich in Wolfenbüttel niedergelassen. Wohl im Jahr 1748 siedelte er nach Göttingen über, wo er wegen seines umfangreichen Kabinetts und zur Unterscheidung von dem Göttinger Botaniker David Sigismund August Büttner, ,Stein-Büttner‘ genannt wurde. ${ }^{314}$ Durch seine umfassenden Kenntnisse der Naturgeschichte, der Anthropologie und Ethnographie sowie seiner umfangreichen Sprachkenntnisse, vor allem aber wegen seiner Sammlungen wurde er in Göttingen schnell bekannt. 1752 wurde er zum außerordentlichen Mitglied der Societät der Wissenschaften ernannt. ${ }^{315}$ Dem folgte 1755 durch die Philosophische Fakultät der Univer-

311 Vgl. UAG Kur 4 V g 1, 4r, und Hildegard StUMPF, Christian Wilhelm Büttner, in: NDB 3 (1957), 6f, 6.

312 Vgl. UAG Kur 4 V g 19, 48r.

313 EHLERS, Göttinger Zoologen, 7f. Ganz anders liest sich dagegen die Darstellung zu Büttner in der NDB. Dort heißt es: Büttner „hatte sich ohne schulgerechtes Studium ein umfassendes Wissen von Naturgeschichte, Anthropologie, Ethnographie und Linguistik angeeignet." STUMPF, Büttner, 6.

314 Vgl. EHLERS, Göttinger Zoologen, 5.

315 Vgl. EHLERS, Göttinger Zoologen, $5 f$. 
sität Göttingen die Ernennung zum Magister. Kurz darauf begann er als königlicher Commissair mit dem Abhalten von Vorlesungen. 1758 wurde er zum außerordentlichen und fünf Jahre darauf zum ordentlichen Professor ernannt.

Blumenbach, der Büttner 1769 kennen gelernt hatte, charakterisierte ihn als „wundersam vielwissende[n] Sonderling, der zumal wegen seiner vielfachen Sprachkenntnisse“ bekannt gewesen sei, und „schon seit langen Jahren keine Collegia gelesen hatte“, aber gerade in Blumenbachs erstem Göttinger Semester wieder ein Kollegium hielt. ${ }^{316}$ Wie bereits erwähnt, gilt Büttner als erster Lehrender, der ein eigenes Kolleg über die Naturgeschichte anbot. Als wissenschaftliche Leistung ist ihm vor allem seine Eigenschaft als Impulsgeber anzurechnen. So beeinflusste er beispielsweise durch seine Abkehr von der „Lehre der unbedingten Konstanz“ und der daraus gezogenen Schlussfolgerung, „die Entwicklung der verschiedenen Rassen aus einer Grundform ableiten zu können“, maßgeblich Blumenbachs berühmt gewordene Dissertation De generis humani varietate nativa, die diesem den Ruf als Begründer der physischen Anthropologie einbrachte. ${ }^{317}$

1783 ging Büttner als Hofrath des Herzogs von Sachsen Weimar nach Jena. ${ }^{318}$ Hierfür scheinen vor allem finanzielle Gründe ausschlaggebend gewesen zu sein. ${ }^{319} \mathrm{Am}$ 8. Oktober 1801 verstarb er 85-jährig in Jena. ${ }^{320}$

\section{Christian Gottlob Heyne}

Der Lebensweg Heynes kann als klassisches Beispiel eines sozialen Aufstiegs herangezogen werden. ${ }^{321}$ Am 25. September 1729 als Sohn eines Leinewebers in Chem-

316 Blumenbach zitiert nach MARX, Andenken an Blumenbach, 5.

317 Beide Zitate aus STUMPF, Büttner, 6. Daneben schreibt Plischke Büttner - wie im Übrigen auch Blumenbach - den Charakter einer „Sammlernatur“ zu. Vgl. PlischKE, Ethnographische Sammlung, 19.

318 Büttners Dienstentlassung in Göttingen ist auf den 10. Oktober 1783 zu datieren. Vgl. PLISCHKE, Ethnographische Sammlung, 12.

319 So heißt es bei EHLERS, Göttinger Zoologen, 7: „Als [Büttner], durch seinen Sammeleifer verschuldet, 1783 seine Bibliothek an den Herzog Carl August von Weimar abtrat, siedelte er in dessen Diensten nach Jena über.“

${ }^{320}$ Mit dem Ordnen seines Nachlasses war augenscheinlich Johann Wolfgang von Goethe betraut. Vgl. EHLERS, Göttinger Zoologen, 7.

${ }^{321}$ Eine etwas ausführlichere Darstellung des Lebenswegs Heynes unter besonderer Berücksichtigung seiner Beziehungen nicht nur zur Universität, sondern auch zur Stadt Göttingen veröffentlichte WOLFGANG BÖKER, Heyne und das Haus, 93-111. Umfassende Auskunft über das Leben Heynes gibt überdies ARnOld Hermann Ludwig HeEREn, Christian Gottlob Heyne. Biographisch dargestellt, Göttingen 1813. In der 430 Seiten langen Abhandlung gibt sein Schwiegersohn ausführlich Auskunft über dessen Leben und Wirken und gestattet Einblicke in dessen Geisteshaltung. 
nitz geboren, wurde es ihm durch die Unterstützung seines Paten ermöglicht, von 1741 ab das Lyzeum zu besuchen. Sein Universitätsstudium der Theologie und Rechtswissenschaft in Leipzig mit anschließender Promotion im Jahre 1752 absolvierte er unter schwierigsten finanziellen Bedingungen. ${ }^{322}$ Nach seinem Studium war Heyne, von 1753 bis 1756 „,als gering bezahlter Kopist“‘323 an der Bibliothek des Grafen Heinrich von Brühl (1700-1763) in Dresden tätig, wo er erste bibliothekarische Erfahrungen sammelte. In diese Zeit fallen auch erste schriftstellerische Versuche, die das Augenmerk bedeutender Wissenschaftler auf Heyne lenkten. Von Oktober 1757 bis 1759 war er mit der Erziehung eines jungen Edelmanns betraut. Im darauf folgenden Jahr zerschlug sich ein viel versprechendes Angebot: Nach Dresden zurückgekehrt, war ihm „die Anwartschaft auf die Stelle des zweiten Bibliothekars an der kurfürstlichen Bibliothek mit der Aussicht auf 500 Taler Gehalt“ angeboten worden. ${ }^{324}$ Doch ,ging bei dem Bombardement der Stadt durch die Preußen [im Juli 1760] seine ganze geringe Habe nebst allen seinen Exzerpten und Vorarbeiten für weitere literarische Arbeiten zu Grunde und er gerieth wieder in bittere Noth“ ${ }^{325}$ Eine dauerhafte Besserung der Verhältnisse trat erst ein, als Heyne im März des Jahres 1763 als Nachfolger Gesners zum Professor der Poesie und Beredsamkeit, Direktor des philologischen Seminars und Bibliothekar an die Universität Göttingen berufen wurde. Von diesem Zeitpunkt an - bis etwa ins Jahr 1790 - nahmen die Zahl seiner Ämter und sein Einfluss stetig zu. ${ }^{326} 1773$ schließlich wurden ihm die Oberaufsicht über das Academische Museum und 1774 das Amt des Inspektors über die Freitische der Universität übertragen. Zudem trat er nach dem Tod seiner ersten Frau durch seine zweite Ehe in verwandtschaftliche Beziehung mit dem im Kurfürstentum Hannover für Universitätsangelegenheiten zuständigen Geheimen Kanzleisekretär

322 Bursian vermerkt, dass Heyne sein Studium durch Unterrichten finanziert habe. Die Art des Unterrichts ist nicht näher spezifiziert. Vgl. ADB, Bd 12, 375.

323 BÖKER, Heyne und das Haus, 95.

324 ADB, Bd 12, 375.

325 ADB, Bd 12, 375. Nichts desto trotz heiratete er am 4. Juni 1761 die ebenfalls aus ärmlichen Verhältnissen stammende Therese Weiß (1730-1775).

326 So wurde er bereits Ende des Jahres 1763 zum Oberbibliothekar und zum ordentlichen Mitglied der Akademie der Wissenschaften ernannt. Ab 1770 wurde er zusätzlich Inspektor des Pädagogiums in Illfeld und übernahm das Amt des ständigen Sekretärs der Akademie der Wissenschaften sowie die Redaktion der Göttingischen Anzeigen von Gelehrten Sachen. Vgl. dazu in aller Ausführlichkeit LUIGI MARINO, Praeceptores Germaniae, 41. Zum Umfang seiner Rezensionstätigkeit vgl. OsCAR FAMBACH (Hg.), Die Mitarbeiter der Göttingischen Gelehrten Anzeigen 1769-1836. Nach dem mit Beischriften des Jeremias David Reuß versehenen Exemplar der Universitätsbibliothek Tübingen, Tübingen 1976, 455-462. 
Georg Friedrich Brandes. ${ }^{327}$ Heynes Wirken trug in zweifacher Weise maßgeblich zum Ruf der Georgia Augusta bei: Zum einen gab er „,[a]ls einer der bedeutendsten klassischen Philologen seiner Zeit [...] mit seinen vor allem auf textliche Authentizität gerichteten Editionen antiker Werke der geistigen Bewegung des Neuhumanismus entscheidende Anstöße“. ${ }^{328}$ Zum anderen erlebte die Universitätsbibliothek unter seiner Leitung einen enormen Reputationsgewinn, was sich nicht nur in einem starken Wachstum der Bestände, sondern auch und vor allem durch ein „ausgereiftes Katalogsystem und durch ,revolutionäre‘, liberale Nutzungsbedingungen“ manifestierte. $^{329}$ Weiter ist es Heyne hoch anzurechnen, dass er „[m]it seinen ArchäologieVorlesungen (seit 1767) [...] die neue Disziplin an der Universität“ verankerte. ${ }^{330}$ Heyne gab die Leitung der Bibliothek 1810 ab. ${ }^{331}$ Es ist sehr wahrscheinlich, dass zu diesem Zeitpunkt die Direktion des Academischen Museums an Blumenbach überging. Heyne starb am 14. Juli 1812 in Göttingen.

\section{Johann Friedrich Blumenbach}

Johann Friedrich Blumenbach ${ }^{332}$ wurde am 11. Mai 1752 als ältester Sohn einer „durch Bildung und Gelehrsamkeit ausgezeichneten Familie Thüringens““333 geboren und von früher Kindheit an gefördert. ${ }^{334}$ Nach dem Besuch des Gothaer Gymnasiums nahm er im Oktober 1769 in Jena das Studium der Medizin auf. Dort hörte er nicht nur Vorlesungen zur Medizin, sondern auch zu Literatur und Bücherkunde, Naturge-

327 Heyne heiratete Georg Brandes’ Tochter Georgine.

328 RUdOlF VIERHAUs/HANS ERICH BÖDEKER, Biographische Enzyklopädie der deutschsprachigen Aufklärung, München 2002, Eintrag „Heyne“, 136.

329 Vgl. BöKER, Heyne und das Haus, 97.

330 VIERHAUS/BÖDEKER, Biographische Enzyklopädie, 136.

331 Vgl. BöKER, Heyne und das Haus, 97.

332 Soweit überschaubar, liegen zur Person Blumenbachs zwar zahlreiche Artikel, jedoch keine i. e. S. wissenschaftliche Biographie vor. Es scheint, als warte er - wie vor wenigen Jahren noch Alexander von Humboldt -, darauf, wiederentdeckt zu werden.

333 F.W.P. DougherTy, Commercium epistolicum J. F. Blumenbachii: Aus einem Briefwechsel des klassischen Zeitalters der Naturgeschichte. Katalog zur Ausstellung im Foyer der Niedersächsischen Staats- und Universitätsbibliothek Göttingen, 1. Juni-21. Juni 1984, Göttingen 1984, 21.

334 Durch seine Mutter, Charlotte Eleonore Hedwig Blumenbach, geborene Buddeus (1727-1794), Tochter des Gothaischen Vizekanzlers und Enkelin des Jenaischen Theologen, wurde Johann Friedrich an die Literatur herangeführt. Sein Vater, Heinrich Blumenbach (1709-1787) wirkte als Professor am Gymnasium Ernestinum in Gotha und konnte laut MARX, Andenken, 51, eine „,auserlesene Bibliothek, viele Kupferwerke und Landkarten“ sein Eigen nennen. Er ließ Johann Friedrich eine frühe Förderung auf dem Gebiet der Naturgeschichte zu Teil werden. Blumenbach genoss eine christlich-evangelisch geprägte, disziplinierte Erziehung. Hg. AdOLF KLEINSCHMIDT, Blumenbach, Johann Friedrich, in: NDB 2 (1955) 329f, 329. 
schichte und Archäologie. ${ }^{335}$ Zum Wintersemester 1772/73 wechselte er nach Göttingen. Das obligate Empfehlungsschreiben überreichte er Heyne, dem er durch sein Auftreten positiv in Erinnerung blieb ${ }^{336}$ - ein Umstand, der ihm später von Nutzen sein sollte. Neben seinen medizinischen Vorlesungen belegte Blumenbach das Kollegium der Naturgeschichte und lernte so den „sonderbaren aber merkwürdigen Büttner“337 kennen:

Das sogenannte Collegium war ein blosses Conversatorium, worin wochenlang von der Naturgeschichte keine Rede war. Doch hatte er die XII. Edit. des Syst. Nat. ${ }^{338}$ als Compendium gewählt, wo wir aber im ganzen Semester unter so hunderterley ganz fremdartigen Dingen, die er einmischte, noch lange nicht mit den Säugethieren durchkamen.

Da er mit den Menschen anfing, den der Jenaische Walch in seinen Vorlesungen unberührt gelassen hatte, und aus seiner zahlreichen Bibliothek eine Menge Abbildungen fremder Völkerschaften herbeibrachte, so reizte mich das, meine Doctordissertation de generis humani varietate nativa zu schreiben, und die weitere Verfolgung dieses interessanten Gegenstandes hat dann den Anlass zu meiner anthropologischen Sammlung gegeben, die mit der Zeit durch ihre in ihrer Art einzige Vollständigkeit allgemein berühmt worden.

Gleich in jenem ersten Winter kam durch Heyne's Vermittlung der Ankauf von Büttners Naturalien- und Münz-Cabinett für die Universität zu Stande. Nur war in der exemplarischen Unordnung, worin die Naturalien bei dem ohnehin nichts weniger als expediten Manne ganz unverzeichnet durcheinander lagen, ihm ein Gehülfe zum Ordnen und Abliefern nöthig. Heyne fragte ihn also: ,Nun Sie lesen ja eben Naturgeschichte; haben Sie denn da unter Ihren jungen Leuten nicht etwa einen, der dazu passt?‘ ,Doch ja', sagte Büttner, und nannte mich. ,I, den kenn' ich auch' und so ward mir diese Hülfeleistung angetragen, die ich lehrreich fand und mit Vergnügen unentgeltlich übernahm.

Einige Zeit nachdem schon allerhand abgeliefert und einstweilen ins vormalige medicinische Auditorium gebracht war, kam der würdige Minister und Curator der Universität, von Lenthe, hieher, besah unsere Institute, und da sollten ihm doch auch diese Sachen gewiesen werden, und weil der ehrliche Büttner dazu nicht eben geeignet schien, so ward ich eilig gerufen, und machte meine Sache so leidlich, dass der Minister gleich beim Herausgehn Heynen bei Seite genommen und gesagt hat: ,Den jungen Mann müssen wir hier behalten. ' - Nachdem ich im Herbst 75 am Anniversarium der Universität promovirt hatte, hielt ich gleich im nächsten Winter als Privatdocent meine ersten Vorlesungen über die Naturgeschichte und ward noch in diesem Semester im Febr. 76 zum ausserordentlichen und hernach im Nov. 78 zum ordentlichen Prof. der Medicin ernannt. $^{339}$

So beschrieb Blumenbach selbst seine Eindrücke. Ein Großteil von ihnen ist durch den Abgleich mit anderen Quellen verifizierbar. Außer diesem Selbstzeugnis Blu-

${ }^{335}$ Letztere las Johann Ernst Immanuel Walch. Er gilt als der Begründer der Jenaischen Sammlungen. Überdies steht er in verwandtschaftlicher Beziehung zu Blumenbach, er war dessen Großcousin. Möglicherweise wurde Blumenbach von Walch in seiner Sammelleidenschaft beeinflusst.

336 Näher hierzu MARX, Andenken an Blumenbach, 5.

337 MARX, Andenken an Blumenbach, 5.

${ }^{338}$ Gemeint ist die 1766-1768 in Stockholm erschienene, 12. Ausgabe von CARL VON LINNÉs Systema naturae per regna tria naturae, secundum classes, ordines, genera, species, cum characteribus, differentiis, synonymis, locis.

339 MARX, Andenken an Blumenbach, 5f. Hervorhebung im Original. 
menbachs belegt jedoch keine andere Quelle die ersten Kontakte zwischen ihm und den Sammlungen des Academischen Museums.

Blumenbachs 1775 beendete Dissertation De generis humani varietate nativa sollte späterhin seinen weltweiten Ruhm begründen. Im Februar 1776 wurde Blumenbach zum außerordentlichen Professor der Medizinischen Fakultät und zum Unteraufseher des Akademischen Museums ernannt. ${ }^{340}$ Neben seiner Tätigkeit als Prosektor führte er die Naturgeschichte-Vorlesungen Büttners fort. Diese sollte er, später auf der Grundlage seines eigenen Handbuchs der Naturgeschichte, ${ }^{341}$ bis 1836 zweimal jährlich halten. Zahlreiche Quellen sprechen von der außerordentlichen Qualität der Blumenbachschen Vorlesungen. ${ }^{342}$

Acht Tage vor seiner Hochzeit mit Luise Amalie Brandes ${ }^{343}$ am 19. Oktober $1778^{344}$ schlug Blumenbachs Schwager in spe Christian Gottlob Heyne dem Geheimen Ratskollegium in Hannover vor, Blumenbach zum ordentlichen Professor zu ernennen und sein Gehalt um 100 Rthlr zu erhöhen. Etwa einen Monat später wurde diesem Gesuch durch ein Reskript Georgs III. stattgegeben. ${ }^{345} 1784$ wurde Blumenbach Mitglied der Göttinger Akademie der Wissenschaften, 18 Jahre darauf, 1812, deren ständiger Sekretär der physikalisch-mathemati-schen Klasse. In Lauf seines Lebens wurde Blumenbach Mitglied von über siebzig Akademien bzw. gelehrten Gesellschaften, darunter des Institut de France, der Londoner Royal Society, der St. Peters-

${ }^{340}$ Vgl. UAG Kur 4 V g 5.

341 Johann Friedrich BlumenBaCH, Handbuch der Naturgeschichte. Mit Kupfern. 2 Bde, Göttingen 1779-1780.

342 Beispielhaft sei hier verwiesen auf den Bericht des Universitätsbereisers Friedrich Gedicke, vgl. VANDENHOECK \& RUPRECHT/DEUERLICHSCHE BuCHHANDLUNG (Hg.), Mehr als irgend eine andere in Deutschland bekannt. Die Göttinger Universität im Bericht des „Universitätsbereisers“ Friedrich Gedicke aus dem Jahre 1789. Mit einer Vorbemerkung von Hartmut Boockmann. Sonderdruck, Göttingen 1996; siehe auch das Schreiben Heynes an das Geheime Ratskollegium in Hannover, in dem er die Berufung Blumenbachs zum ordentlichen Professor vorschlug. Vgl. DougHERTY, Commercium epistolicum, 27.

343 Die Bedeutung dieser Heirat ist - sowohl in wissenschaftlicher als auch in politischer Hinsicht nicht zu unterschätzen. Durch sie wurde Blumenbach nicht nur Schwager Heynes, sondern auch Schwiegersohn Georg Brandes', der von Hannover aus starken Einfluss auf die Universität nahm. Es ist wahrscheinlich, dass dadurch Blumenbachs Position an der Universität entscheidend gestärkt wurde.

344 Bei der Datierung der Hochzeit beziehe ich mich auf MARX, Andenken an Blumenbach, 53, und DOUGHERTY, Commercium epistolicum, 219. KLEINSCHMIDT, Blumenbach, 329 und DougHERTY, Commercium epistolicum, 21 [sic], geben als Hochzeitstermin erst den 19. Oktober 1779 an, was den oben geschilderten Vorgängen einiges an Brisanz nehmen würde.

345 Vgl. DOUGHERTY, Commercium epistolicum, $27 \mathrm{f}$. 
burger Akademie und der American Philosophical Society. Neben zahlreichen anderen Ehrungen wurde ihm 1816 der Titel eines Obermedizinalrates verliehen.

Blumenbach hatte ein breit gestreutes Interessenspektrum, dessen einzelne Komponenten sich in seinen Arbeiten wiederfinden lassen. Von Haus aus Mediziner, war er an Sammlungen geschult und arbeitete zudem viel mit Reise- und Forschungsberichten. ${ }^{346}$ Dabei blieb er einem „naturphilosophisch geleiteten systematischen Denken und Ordnen“347 verpflichtet. Durch die Einbeziehung entwicklungs- und entstehungsgeschichtlicher Gesichtspunkte in biologische Klassifikationssysteme ${ }^{348}$ leistete er einen maßgeblichen Beitrag zur Verzeitlichung der Taxonomien.

Wissenschaftliche Berühmtheit erlangte Blumenbach v.a. als Begründer „einer physischen Anthropologie, die sich auf anatomische und physiologische Merkmale stützt, aber auch auf ethnologisch-kulturelle Zusammenhänge verweist““. ${ }^{349}$ In diesem Zusammenhang gilt er ebenso als Begründer der vergleichenden Anatomie. ${ }^{350}$ Bereits in seiner Dissertation hatte Blumenbach eine Lehre von der „Entfaltung der Menschheit in der Vielheit von Rassen und Varietäten““351 entwickelt. Dabei soll er sich vor allem an seinem Lehrer Büttner sowie den Schriften Kants orientiert haben. ${ }^{352}$ Mit der stark erweiterten dritten Auflage dieser Schrift (1795) erlangte Blumenbach internationale wissenschaftliche Anerkennung. Darin unterschied er fünf Menschenrassen (Kaukasisch, Mongolisch, Äthiopisch, Amerikanisch, Malaiisch), die sich aus einer Urrasse entwickelt hätten und gleichwertig nebeneinander stünden. Grundgedanke war, dass sich diese Rassen aufgrund klimatologischer Faktoren, ver-

346 Durch Blumenbachs Lehrtätigkeit einerseits und seine intensive Beschäftigung mit Reisenberichten andererseits entstand eine wechselseitige Beeinflussung mit den Forschungsreisenden seiner Zeit. Hier sind vor allem Alexander von Humboldt, Friedrich Hornemann und Prinz Maximilian zu Wied Neuwied zu nennen. Plischke widmet diesem Themenkomplex eine eigene Abhandlung. Vgl. HANS PLISCHKE, Johann Friedrich Blumenbachs Einfluß auf die Entdeckungsreisenden seiner Zeit, Göttingen 1937. Allerdings ist darin insbesondere der Rassebegriff nationalsozialistisch eingefärbt.

347 Fritz HARTMAnN, Johann Friedrich Blumenbach, in: Rudolf Vierhaus/Hans Erich Bödeker (Hg.), Biographische Enzyklopädie der deutschsprachigen Aufklärung, München 2002, 36.

348 Blumenbach stand Linné kritisch gegenüber. Er erweiterte und ergänzte dessen Systematik vor allem in Bezug auf das Tierreich.

349 HARTMANN, Blumenbach, 36.

350 Laut der Internetseite http://www.whonamedit.com/doctor.cfm/1247.html (Zugriff am 16. November 2004), war Blumenbach der erste, der Vorlesungen in vergleichender Anatomie hielt.

351 HARTMANN, Blumenbach, 36.

352 Vgl. den Abschnitt zu Büttner in diesem Kapitel; ferner IMMANUEL KaNT, Von den verschiedenen Racen der Menschen. Zur Ankündigung der Vorlesungen der physischen Geographie im Sommerhalbenjahre 1775, Königsberg 1775. 
schiedener Ernährungsweisen und anderer Umwelteinflüsse ausdifferenziert hätten. Die Unterscheidungen traf er anhand von „Schädelgestalt, Zähne[n], Konstitution“. ${ }^{353}$ In diesen Kontext ist auch seine berühmt gewordene Schädelsammlung einzuordnen.

Überdies trat Blumenbach mit seinem Handbuch der Naturgeschichte hervor, in dem er den Menschen eindeutig den Säugetieren zuordnete, jedoch auch den Unterscheidungskriterien der beiden Gruppen große Aufmerksamkeit widmete. ${ }^{354}$ Zudem geht auf ihn der „die gestalterische Kraft der Embryonalentwicklung““355 beschreibende Begriff nisus formativus (Bildungstrieb) zurück, wodurch Blumenbach „zu den naturphilosophisch argumentierenden Vitalisten gerechnet wird. Jedoch unterschied [...; er] scharf die mechanistischen von den lebendigen, zielstrebigen Kräften.“356 $\mathrm{Ob}$ man Blumenbach überdies das Verdienst zuschreiben darf, maßgeblichen Anteil an der Popularisierung der Wissenschaften genommen zu haben, wie Marx dies andeutet, bleibt zu untersuchen. ${ }^{357}$ Blumenbach starb hoch geschätzt im Alter von 87 Jahren am 22. Januar 1840 in Göttingen.

\section{Friedrich Albert Anton Meyer}

Friedrich Albert Anton Meyer wurde am 29. Januar 1769 in Harburg geboren. Er studierte in Göttingen und „ward daselbst Magister der Philosophie und Doctor der Arzneikunde Ostern 1792“. ${ }^{358}$ Im selben Jahr wurde er Privatdozent an der Medizinischen Fakultät ${ }^{359}$ und von Ende 1792 an war er Unteraufseher am Academischen Museum. ${ }^{360}$ Über sein Aufgabenfeld im Museum oder auch sein (Hierarchie-) Verhältnis zu Blumenbach ist nichts bekannt. Ehlers schreibt Meyer vor allem das Verdienst zu, „zuerst in Göttingen die Zoologie als Einzelfach in den Lehrplan“361 ein-

\footnotetext{
353 HARTMANN, Blumenbach, 36.

354 BlumenBACH, Handbuch der Naturgeschichte, 2 Bde, Göttingen 1779/80.

355 HARTMANN, Blumenbach, 36.

356 HARTMANN, Blumenbach, 36.

357 Vgl. MARX, Andenken an Blumenbach, 7. Dort konstatiert dieser, „dass er [Blumenbach; CN] es vorzüglich war, der die Naturkunde in unserem Vaterlande aus den engen Räumen der Bücher und Museen in den weiten, heitern Kreis des Lebens hereinzog; dass er die Resultate ernster, einsamer Forschung jedem Lernbegierigen und Gebildeten verständlich und geniessbar machte, und besonders die höhere Gesellschaft dafür zu interessieren, ja zu begeistern verstand.“

358 SAALFELD, Gelehrten-Geschichte (3), 162.

359 Vgl. EHLERS, Göttinger Zoologen, 17.

360 Vgl. SAALFELD, Gelehrten-Geschichte (3), 162.

361 EHLERS, Göttinger Zoologen, 17.
} 
geführt zu haben. Meyer veröffentlichte vor allem naturgeschichtliche, ethnologisch ausgerichtete Schriften mit den Schwerpunkten Mineralogie und Entomologie. ${ }^{362} \mathrm{Er}$ starb 26-jährig am 29. November 1795.

\section{Johann Ludwig Gravenhorst}

Johann Ludwig Gravenhorst ${ }^{363}$ wurde am 14. November 1777 als Sohn eines wohlhabenden Brauereibesitzers in Braunschweig geboren. Nach seiner Schulzeit in Braunschweig begann er in Helmstedt mit dem Studium der Rechtswissenschaft, siedelte jedoch nicht lange darauf nach Göttingen um und widmete sich dort von 1799 an dem Studium der Naturwissenschaft. „1801 promovirte [sic] er in Helmstedt als doctor philosophiae et magister liberalium artium“364 mit einer entomologischen Arbeit. Bei einer Forschungsreise nach Paris im darauf folgenden Jahr trat er mit den führenden Entomologen seiner Zeit in Kontakt. Seit 1804 hielt er in Göttingen als Privatdozent Vorlesungen „über besondere Theile der Naturgeschichte“ ${ }^{365}$ Gegen Ende des Jahres 1808 wurde er zum außerordentlichen Professor ernannt und „bei der Aufsicht des akademischen Museums adjungirt““ ${ }^{366}$ Bereits zwei Jahre später verließ er Göttingen, um einem Ruf als ordentlicher Professor nach Frankfurt/Oder zu folgen, von wo aus er 1811 nach Breslau ging. Dort überließ er noch zu Lebzeiten der Universität seine umfangreiche Naturaliensammlung, die den Grundstock für das zoologische Museum der Universität bildete, deren erster Direktor er wurde. ${ }^{367}$ So trug Gravenhorst seine in Göttingen gewonnenen museumspraktischen Erfahrungen nach Breslau weiter. Wissenschaftlich blieb Gravenhorst als Schüler Blumenbachs stets der vergleichenden Anatomie bzw. der systematischen Zoologie verbunden. Dabei lag sein Interessenschwerpunkt vor allem auf Insekten und Reptilien. Gravenhorst starb am 14. Januar 1857 in Breslau.

362 Eine Auflistung davon sowie eine Übersicht über seine Vorlesungsinhalte findet sich bei SAALFELD, Gelehrten-Geschichte (3), 161-164.

363 Verschiedene Autoren geben unterschiedliche dritte Vornamen für Gravenhorst an, weshalb in der Überschrift nur die beiden Übereinstimmenden genannt sind. So heißt er bei CARUS, Gravenhorst, in ADB 9 (1879), 616 Johann Ludwig Christian, bei EHLERS, Göttinger Zoologen, 21 jedoch Johann Ludwig Christoph.

364 EHLERS, Göttinger Zoologen, 22.

365 EHLERS, Göttinger Zoologen, 22.

366 EHLERS, Göttinger Zoologen, 23. Wahrscheinlich handelte es sich um die Position eines Unteraufsehers.

367 Vgl. EHLERS, Göttinger Zoologen, 25. 


\section{Johann Friedrich Osiander}

Osiander wurde am 2. Februar 1787 in Kirchheim unter Teck in Württemberg geboren. Von 1804 bis 1808 absolvierte er in Göttingen ein Medizinstudium, das er mit einer Promotion abschloss. Überdies „erhielt [er] 1808 den medicinischen Preis““. ${ }^{368}$ Nach seiner Ernennung zum Doktor der Medizin am 1. Oktober 1808, begab er sich auf eine wissenschaftliche Reise. Nach Göttingen zurückgekehrt, ${ }^{369}$ übernahm es Osiander, Blumenbach von 1812 ab bei der Erstellung eines von Seiten der westphälischen Regierung angeforderten Inventars der Cook-Forster-Sammlung zu unterstützen. ${ }^{370} 1815$ wurde ihm offiziell die (Mit-)Aufsicht über die zoologische und ethnographische Sammlung des Museums übertragen, ${ }^{371}$ die er mit Unterbrechungen bis zu seinem Tod innehaben sollte. Ebenfalls 1815 wurde er zum außerordentlichen Professor der Medizin ernannt. ${ }^{372}$ Sein Schwerpunktfach war die Geburtshilfe, jedoch trat er nie aus dem Schatten seines Vaters heraus. ${ }^{373} 1827$ wurde Osiander zum fürstlich waldeckischen Hofrath, und 1833 zum ordentlichen Professor der Medizin ernannt. ${ }^{374}$ Zudem war Osiander seit 1810 Assessor der Göttinger Akademie der Wissenschaften und stand mit weiteren Akademien in Korrespondenz. ${ }^{375}$ Osiander starb am 10. Februar 1855.

\footnotetext{
368 SAALFELD, Gelehrten-Geschichte (3), 379.

369 Für die dazwischen liegenden sieben Jahre sind die Angaben in der Literatur etwas widersprüchlich: Während SAALFELD, Pütters Gelehrten-Geschichte (3), 379, schreibt: „War auf Reisen von dem J. 1808 bis 1810 zu Tübingen, Paris, Strasburg, und 1814 in Wien, Doctor seit 1808, Privatdocent seit 1811, seit 1816 ausserordentlicher Professor und Mitaufseher des zoolog. Theils des königl. Museums“, heißt es knapp zwanzig Jahre später bei OESTERLEY, Gelehrten-Geschichte (4), 432, dass Osiander bereits 1810 Privatdozent und 1815 Mitaufseher des Museums geworden sei. Bei SAALFELD, Gelehrten-Geschichte (3), 379, findet sich zusätzlich ein Verzeichnis der von Osiander bis zum Jahr 1820 verfassten Schriften sowie eine Angabe über die von ihm gehaltenen Vorlesungen.

370 Dieses Verzeichnis mit dem Titel (Kunstsachen) Handarbeiten und Naturalien aus den Südsee Inseln befindet sich in den Beständen des Archivs der Völkerkundlichen Sammlung Göttingen. Im Rahmen dieser Arbeit wurde jedoch nicht darauf zugegriffen.

371 Vgl. OESTERLEY, Gelehrten-Geschichte (4), 432.

${ }^{372}$ Vgl. UAG Kur 4 V g 5, bes. 38f.

373 Osianders Vater Friedrich Benjamin Osiander (1759-1822), wirkte von 1792 bis 1822 als Professor an der Medizinischen Fakultät der Georgia Augusta und galt als Koryphäe auf dem Gebiet der Geburtshilfe.

374 OESTERLEY, Gelehrten-Geschichte (4), 432.

375 Vgl. OESTERLEY, Gelehrten-Geschichte (4), $432 \mathrm{f}$.
} 


\section{Johann Friedrich Ludwig Hausmann}

Hausmann wurde am 22. Februar 1782 in Hannover geboren. Dem Schulbesuch in Hannover und Braunschweig folgte das Studium der Rechtswissenschaften, Mineralogie, Chemie und Technologie in Göttingen. Als Hausmann 1815 mit der Verwaltung der mineralogischen Sammlung des Academischen Museums betraut wurde, konnte er auf langjährige Erfahrungen im Bereich der Mineralogie und der Montanwissenschaften zurückblicken. Diese schließen die verschiedensten Ämter im Bergund Hüttenwesen in Braunschweig und dem Königreich Westfalen ${ }^{376}$ ebenso ein wie wissenschaftliche Reisen ${ }^{377}$ und die Gründung der noch heute als TU bestehende Lehranstalt für das Bergwesen in Clausthal. Nach dem Tod Beckmanns 1811 hatte er an der Universität Göttingen dessen Lehrstuhl für Technologie und Bergwissenschaft übernommen, den er 48 Jahre innehaben sollte. Im selben Jahr wurde er in die Göttinger Akademie der Wissenschaften aufgenommen, acht Jahre später erfolgte seine Ernennung zum Hannoverschen Hofrat. Für das Academische Museum war die Mitarbeit Hausmanns eine große Bereicherung. Durch seine Anstellung erhielt die umfangreichste der Sammlungen des Academischen Museums einen erwiesenermaßen fachkompetenten Betreuer. Unter seinen wissenschaftlichen Tätigkeiten und Publikationen ist im Hinblick auf seine Berufung für das und seine Tätigkeit im Museum besonders sein Handbuch der Mineralogie hervorzuheben, mit dem er bereits 1809 die Grundlage für eine Systematik der Mineralien aufgrund ihrer „natürlichen chemi-

3761803 nahm Hausmann eine Stelle als Bergamts-Auditor der Bergämter Clausthal und Zellerfeld an und arbeitete gleichzeitig als Bergmeister auf zwei Gruben. Von dort aus wurde Hausmann 1805 zum Kammersekretär des Berg- und Hüttendepartements in Braunschweig berufen. 1808 erhielt er einen Posten als Sekretär der Berghauptmannschaft, den er jedoch im Zuge der napoleonischen Herrschaft verlor. Der Versuch, sich in Göttingen um eine Professur der Bergwerkswissenschaft zu bewerben, scheiterte zunächst, so dass er gezwungen war, ein anderweitiges Auskommen zu suchen. In den Jahren 1809 bis 1811 bekleidete er die Ämter des Generalsekretärs im Finanzministerium und des Generalinspektors der Berg-, Hütten-, und Salinenwerke des Königreichs Westphalen. In dieser Zeit setzte er verschiedene Reformen im Montanwesen durch.

377 In den Jahren 1806 bis 1808 reiste er zu Wissenschaftszwecken durch Skandinavien. Weitere Forschungsreisen fallen in die Jahre 1818/19 (Italien) und 1828/29 (Holland, Belgien, Frankreich, England, Spanien). Darüber hinaus unternahm er alljährlich - teils allein, teils mit Studenten - Exkursionen im Raum Deutschland - Österreich - Schweiz. 
schen und physikalischen Verwandtschaftsverhältnisse“،378 ${ }^{37 h u f . ~ D i e s e ~ b a u t e ~ e r ~ i n ~}$ erneuten Auflagen dieses Werkes 1813, und besonders 1829 systematisch aus. ${ }^{379}$ Heute gilt Hausmann als zudem Begründer der rationalen Bodenkunde, ${ }^{380}$ machte er doch nicht nur zahlreiche Entdeckungen zur Entstehung der Mineralien, zu Untersuchungs- und Beschreibungsmethoden von Mineralienarten und neuen Kristallformen, sondern wandelte diese geologischen Erkenntnisse auch in praktische Anweisungen für Landwirtschaft und Forstwesen um. ${ }^{381}$ Dank seines Engagements wurde der zu Anfang der 1830er Jahre vom Untergang bedrohte Bergbau im Harz weitergeführt, was die Existenz zahlreicher Harzbewohner sicherte. Seine Monographie Der Hannoversche Harz wurde zum Standardwerk der Natur- und Landschaftskunde. ${ }^{382}$

Nach Blumenbachs Tod 1840 übernahm Hausmann dessen Nachfolge als Sekretär der Akademie der Wissenschaften. 1845 wurde er zum Geheimen Hofrat ernannt. Hausmann starb am 26. Dezember 1859 in Göttingen.

\section{August Murray}

August Murray entstammte einer bekannten Göttinger Gelehrtenfamilie. Er wurde am 4. Januar 1797 als Sohn des Universitätsapothekers geboren und begann 1812 das Studium der Medizin in Göttingen, unterbrach dieses jedoch 1813 für eine Karriere beim Militär, die er erst 1818 beendete. ${ }^{383}$ Im Anschluss daran setzte er sein Medizinstudium in Göttingen fort, wo er angeblich bereits im Herbst 1819 promoviert wurde. $^{384}$ Nach einem Aufenthalt in Wien mit anschließender Ungarnreise ließ er sich im darauf folgenden Jahr vorübergehend nahe Göttingen als praktischer Arzt nieder. ${ }^{385}$ Am 31. Januar 1827 wurde er schließlich als Assistent an der zoologischen und ethnographischen Sammlung des Academischen Museums angestellt. Allerdings verließ er diese Stelle nach Jahresfrist, um sich als Arzt in Herzberg niederzulassen,

\footnotetext{
${ }^{378}$ WALTHER FISCHER, Johann Friedrich Ludwig Hausmann, in: NDB 8, 124f, 124.

379 Über weitere wissenschaftliche Werke Hausmanns und damit weitere Forschungsschwerpunkte informiert umfassend der ihm gewidmete Artikel in der ADB. Vgl. GüMBEL, Hausmann, Joh., Friedr. Ludwig, in: ADB 11, 94-97.

380 Vgl. FisCHER, Hausmann, 124f, 125.

381 Vgl. http://www.gfn-harz.de/sites/orte-namen.htm (Zugriff am 11. Januar 2005).

${ }^{382}$ Vgl. http://www.gfn-harz.de/sites/orte-namen.htm (Zugriff am 11. Januar 2005).

383 Zuerst als Freiwilliger im Range eines Cadetsergeanten in das Lauenburger Feldbataillion eingetreten, wurde Murray bald Lieutenant im Bataillion Münden und nahm als solcher an der Schlacht bei Waterloo (18. Juni 1815) teil.

384 Vgl. EHLERS, Göttinger Zoologen, 90, EN 50.

385 Vgl. EHLERS, Göttinger Zoologen, 90, EN 50.
} 
wo er heiratete. Nach dem Tod seiner Frau 1832 kehrte er nach Göttingen zurück: „Auf seinen dringenden Wunsch wurde er 1843 wiederum als Assistent am akademischen Museum beschäftigt; als solcher 1844 angenommen und 1861 mit dem Titel Inspector angestellt.““386 Murray starb am 13. August 1865.

\section{Ernst Friedrich Gustav Herbst}

Herbst wurde am 5. Januar 1803 in Uslar geboren. Nach dem Schulbesuch in Uslar und Göttingen nahm er 1818 in Göttingen das Studium der Medizin auf, das er 1823 mit Promotion abschloss. 1826 wurde er „Accessist bei der K. Bibliothek“387 und trat drei Jahre später die Nachfolge Murrays als Assistent beim Academischen Museum an. ${ }^{388}$ Bis dahin hatte er durch seine Arbeiten zwei Preise gewonnen: 1822 einen von der medizinischen Fakultät ausgesetzten Preis ${ }^{389}$ und 1827 „den Preis der physischen Classe der hiesigen Societät der Wissensch[aften]“. ${ }^{390}$ Darüber hinaus erhielt er das 1833 erstmalig ausgelobte Stipendium Blumenbachianum für eine Reise nach England, Frankreich und in die Niederlande. Wohl noch im gleichen Jahr ${ }^{391}$ wurde er Sekretär der Universitätsbibliothek. 1836 wurde er Assessor bei der Akademie der Wissenschaften und 1842 wurde er zum außerordentlichen Professor ernannt. Herbst starb am 2. März 1893 in Göttingen.

\section{Arnold Adolph Berthold}

Berthold wurde am 26. Februar 1803 als Sohn eines Tischlermeisters in Soest geboren. Er studierte in Göttingen Medizin und wurde dort im Jahre 1823 promoviert. In den Jahren 1824 und 1825 war er bei Chr. W. Hufeland ${ }^{392}$ in Berlin angestellt und betrieb vergleichend-anatomische und zoologische Studien in Paris. ${ }^{393} 1825$ habili-

386 EHLERS, Göttinger Zoologen, 90, EN 50.

387 Vgl. OESTERLEY, Gelehrten-Geschichte (4), 489.

388 Allerdings ist nicht bekannt, wie lange er diese Position innehatte.

389 Dieser wird bei OESTERLEY, Gelehrten-Geschichte (4), 489, nicht näher spezifiziert und auch die Angabe bei EHLERS, Göttinger Zoologen, 32, ist nicht ganz eindeutig. .

390 OESTERLEY, Gelehrten-Geschichte (4), 489; ebenso EHLERS, Göttinger Zoologen, 32, wo zudem zu erfahren ist, dass der Preis „,[f]ür eine Untersuchung über Benutzung von ,Lungenmessern“ und deren Werth“ vergeben worden war.

391 Vgl. EHLERS, Göttinger Zoologen, 32.

392 Christoph Wilhelm Hufeland (1762-1836) war ein einflussreicher Mediziner. Er gründete die erste Poliklinik in Berlin und gilt heute zudem als Vater der Makrobiotik. Darüber hinaus war er der Leibarzt Friedrich Wilhelms III. und behandelte auch Goethe, Schiller, Herder und Wieland.

393 Vgl. Fritz Bolle, Berthold, Arnold, Adolph, in: NDB 2, 166. 
tierte er sich in Göttingen und „liess sich hier als practischer Arzt und Privatdocent in der medicinischen Fakultät nieder.“394 Zehn Jahre später wurde er zum außerordentlichen, 1836 zum ordentlichen Professor ernannt und 1837 in die Göttinger Akademie der Wissenschaften aufgenommen. Auf wissenschaftlicher Ebene setzte er sich vor allem mit zoologischen und physiologischen Problemen auseinander. ${ }^{395}$ Zudem gilt Berthold heute als Begründer der Hormonforschung. ${ }^{396}$

1836 wurde er mit der Mitaufsicht über die zoologische Sammlung des Academischen Museums betraut, um die er sich besonders verdient machte. Schon ein Jahr später bewirkte er für das Museum die Einrichtung eines festen, wenn auch niedrigen, Jahresetats ${ }^{397}$ - ein wichtiger Fortschritt, stellte diese Neuerung doch einen Schritt zu einer finanziellen Absicherung dar. Nach Blumenbachs Tod übernahm er die alleinige Leitung der zoologischen Sammlung und verstand es, sie geschickt auszubauen und zu bereichern. Er starb am 3. Februar 1861 in Göttingen.

\section{Friedrich August Schlotthauber}

Über Schlotthauber existieren nur vergleichsweise wenige Informationen. Ehlers charakterisiert ihn als „Sonderling“, als „Naturforscher von Göttingen“, der „in den Tageblättern mancherlei naturgeschichtliche Notizen brachte““. ${ }^{398}$ Scheinbar hatte Schlotthauber sich nach dem Schulbesuch in Einbeck und Northeim zum Sommersemester 1826 in Göttingen für Theologie eingeschrieben, ohne ein gymnasiales Abschlusszeugnis vorweisen zu können. „Als Theologiebeflissener wird er bis zum Jahre 1831 in den Personalverzeichnissen geführt.“" ${ }^{399}$ Danach scheint er einige Zeit in seinem Heimatort Edesheim gelebt zu haben. „Von Michaelis 1834 bis Michaelis 1839 ist Schlotthauber wieder in Göttingen immatriculirt, nun aber als Studierender der Naturwissenschaften. “ ${ }^{400}$ In dieser Zeit versah er verschiedentlich Hilfsdienste

\footnotetext{
394 EHLERS, Göttinger Zoologen, 35.

395 Vgl. BoLLE, Berthold, 166.

396 Als wegbereitend gilt sein Aufsatz zur „Transplantation der Hoden“ im Archiv für Anatomie, Physiologie und wissenschaftliche Medizin (1849), 42-46. Eine Aufzählung weiterer Schriften findet sich bei August HIRsch, Berthold, Arnold Adolf, in: ADB 2 (1875), 512.

397 Vgl. EHLERS, Göttinger Zoologen, 38, wo es heißt: „,[D]amals wurde für alle darin vereinigten Sammlungen die Summe von 100 Thlr. jährlich bestimmt, da durchschnittlich jährlich soviel dafür verausgabt sei‘. Dieser Fond war auf Berthold’s Vorstellung 1838 auf 300 Thlr. erhöht.“

398 Vgl. EHLERS, Göttinger Zoologen, 33.

399 EHLERS, Göttinger Zoologen, 33.

400 EHLERS, Göttinger Zoologen, 34
} 
am Museum, die von der Aufsicht während der Öffnungszeiten des Museums bis zur Präparation von Tieren reichten. ${ }^{401}$ Im Jahr 1839 richtete er ein erstes Gesuch an das Museum, in dem er darum bat dort als „Conservateur“ angestellt zu werden. ${ }^{402}$ Scheinbar führte er weiterhin gelegentlich Arbeiten für das Academische Museum aus und erhielt dafür Beihilfen, wurde jedoch nicht seinem Wunsch nach angestellt. ${ }^{403}$ Doch scheint er in Göttingen und darüber hinaus für seine naturgeschichtlichen und besonders auch botanischen Kenntnisse bekannt gewesen zu sein: Nach Ehlers „ertheilte ihm die philosophische Fakultät der Universität Jena 1860 das Doctor-Diplom honoris causa, und von der k. k. zool. bot. Gesellschaft in Wien wurde er 1861 zum Mitgliede gewählt.“404 Schlotthauber starb am 10. November 1872 in Göttingen.

\section{Rudolf Wagner}

Rudolf Wagner wurde am 30. Juli 1805 als Sohn eines königlich bayrischen Hofrats und Studiendirektors am Gymnasium in Bayreuth geboren. Nach dem Schulbesuch in Bayreuth und Augsburg begann er 1822 mit dem Studium der Medizin in Erlangen. 1824 erfolgte ein Studienortswechsel nach Würzburg, wo er 1826 zum Doktor der Medizin promoviert wurde. 1827 folgte ein achtmonatiger Aufenthalt bei $\mathrm{Cu}-$ vier $^{405}$ in Paris, wo er seine Kenntnisse in vergleichender Anatomie vertiefte. Im Anschluss daran führte ihn eine Forschungsreise über die maritime Tierwelt in die Normandie und an die Mittelmeerküste. ${ }^{406} 1828$ ließ er sich vorübergehend als praktischer Arzt in Augsburg nieder, nahm aber schon 1829 eine Stelle als Prosektor in der Anatomie der Universität Erlangen an, wo er sich noch im selben Jahr habilitierte. Bereits drei Jahre später, lehrte er dort als ordentlicher Professor für vergleichen-

401 Vgl. UAG Kur 4 V g 20, 143, 155, 161.

402 Vgl. UAG Kur 4 V g 43.

403 Dies hinderte ihn jedoch nicht daran bis einschließlich 1861 immer wieder Gesuche beim Museum einzureichen, in denen er darum bat, ihm eine Assistentenstelle zu überlassen. Vgl. UAG Kur 4 V g 43.

404 EHLERS, Göttinger Zoologen, 34.

405 Georges Baron de Cuvier (1769-1832) war ein bedeutender Zoologe und Paläontologe - er kann in gewisser Weise als der französische Blumenbach gelten. Er wurde vor allem durch sein auf vergleichender Anatomie beruhendes Klassifikationssystem für Tiere und die von ihm aufgestellte Katastrophentheorie bekannt.

406 Vgl. PAGEL, Wagner, Rudolf, in: ADB 40, 573f, 573. 
de Anatomie und Zoologie ${ }^{407}$ und übernahm die Verwaltung der zoologischen Sammlung der Universität. ${ }^{408}$

In Göttingen sollte Wagner in mehrfacher Hinsicht die Nachfolge Blumenbachs antreten. 1840 wurde er auf dessen Stelle als ordentlicher Professor der Physiologie, vergleichenden Anatomie und Zoologie berufen. Zudem folgte er Blumenbach widerstrebend in der Direktion der zoologischen Sammlung des Academischen Museums nach. Diese entsprach keinesfalls seinen Erwartungen. Er fällte im Oktober 1840 ein vernichtendes Urteil darüber:

\begin{abstract}
,In der That ist die hiesige Sammlung ein Ueberbleibsel aus jener Zeit, wo die Naturgeschichte ein blosser Raritätenkram war, und die zoologische Abtheilung des Museums mit sammt der Blumenbachschen Sammlung sind um 50 Jahre hinter den Bedürfnissen der Zeit zurückgeblieben. ' Er verbittet sich dann jede Theilnahme an der Direction der zoologischen Sammlung: ,Ich müsste mich‘, schreibt er, ,in der That vor durchreisenden fremden Gelehrten schämen, Vorsteher einer Sammlung zu sein, welche mit Ausnahme der Blumenbachschen Schädelsammlung und einzelnen Stücken wirklich eher den Namen einer naturhistorischen Rumpel- und Polterkammer, wie sie Prof. Lichtenstein in Berlin nannte, verdient, denn eines wissenschaftlich geordneten akademischen Museums. ${ }^{409}$
\end{abstract}

Wagner starb am 13. Mai 1864.

\title{
5.1.2 Die Museumswärter
}

Neben dem bisher erwähnten administrativen Personal war für reibungslose Abläufe innerhalb des Museums die Gruppe der Museumswärter von großer Bedeutung. Im Untersuchungszeitraum 1773-1840 lassen sich drei Wärter nachweisen, die im Folgenden ebenfalls kurz vorgestellt werden sollen. ${ }^{410}$ Der erste Wärter, Johann Ludwig Lorenz, war möglicherweise schon seit 1773, sicher jedoch seit 1776 am Museum beschäftigt. Den Wärtern oblagen vor allem konservatorische Aufgaben - dazu gehörten die Suche nach Ungeziefer an den ausgestopften Tieren und Ethnographica, das Bestreichen der ersteren mit Sublimat ${ }^{411}$ sowie die Reinhaltung der Sammlung, wie aus einer Rechnung des Aufsehers Stiepel für das Sommerhalbjahr ${ }^{412} 1814$ hervorgeht. Dort sind als Einzelposten aufgeführt:

407 Vgl. EHLERS, Göttinger Zoologen, 42; PAGEL, Wagner, 573, schreibt hingegen: „Im Frühjahr 1832 bereiste W. Triest, wurde 1833 außerordentlicher Professor der Zoologie.“

408 Vgl. EHLERS, Göttinger Zoologen, 42.

409 Wagner, zitiert nach EHLERS, Göttinger Zoologen, 43f.

410 Bei den im Folgenden gemachten Angaben beziehe ich mich auf WAGENER, Pedelle, 524-528.

411 Bei Sublimat handelt es sich um Quecksilber-II-Chlorid. Es wurde v.a. als Desinfektionsmittel verwendet..

412 Dies umfasst den Zeitraum vom Osterfest bis zum Namenstag von St. Michael am 29. September. 
In den Naturalien-Säälen das trockene Thierreich gereiniget und wo es nöthig mit Sublimat bestrichen. küttet.

Die Spiritus-Gläser nachgesehen, wo es nöthig geöfnet, nachgefüllt und wieder ver-

Die ausgestopften Vögel und Thiere oftmals nachgesehen, gereiniget und mit Sublimat bestrichen.

Die Süd- und Nord-Amerikanischen Kleidungsstücke, Federputze, Trauerputz, Schaman, so wie die Tougusischen, Samojadischen und Kamtschadalischen Pelze und die dazu gehörigen Geräthschaften gereiniget und die Pelze mehrmals mit Sublimat bestrichen.

In den Mineralien- und Naturalien-Säälen beständige Reinigung zu erhalten, und für sonstige vorgefallene nothwendige Arbeiten. ${ }^{413}$

Für diese Arbeiten stellte Stiepel 9 Rthlr 16 gg Kassenmünze in Rechnung, die wohl zu seinem Grundverdienst aufgeschlagen sein worden dürften. ${ }^{414}$ Weitere Zuschläge sind denkbar für die Anwesenheit bei Vorlesungen, ${ }^{415}$ in denen Museumsobjekte demonstriert wurden, oder durch Trinkgelder von Besuchern, durch die ein engagierter Wärter seinen Verdienst erheblich aufbessern konnte. ${ }^{416}$

Weiterhin gehörte es zum Aufgabenbereich der Wärter - und das erscheint aufgrund fehlender fachwissenschaftlicher Kompetenz ungewöhnlich - Besucher durch die Sammlungen zu führen. ${ }^{417}$ Dies belegt Wagener anhand des bereits zuvor erwähnten Diebstahls der Silberstufe im Jahr 1783, die noch in die Dienstzeit des ersten Wärters Lorenz fällt. Blumenbach berichtete darüber:

Donnerstag den $16^{t}$ Jan. gegen 11 U. kommt Lorenz zu mir, fragt ob er einigen Fremden, die ihn darum ersucht, das Museum zeigen dürfe? conceditur. Halb zwölf kommt er ganz ausser sich mit der Nachricht zurück, die grosse Silberstufe sey aus dem Kasten entwendet, wie er soeben, da er sie jenen Fremden zeigen wollen, gewahr geworden. Vorgestern ( $\hat{\sigma}$ den 14ten) habe er sie noch dem Syndicus von Osterode und dessen Gesellschaft gezeigt. $^{418}$

Silke Wagener schlussfolgert daraus, „daß Besuche auf dem Museum recht häufig waren, ferner, daß der Wärter, der die Führungen allein vornahm, seine Schätze sehr

413 UAG Kur 4 V g 20, 16r.

414 WAGENER, Pedelle, 353, gibt das Gehalt Lorenz' für das Jahr 1809 mit 30 Rthlr an. Dieser Verdienst reichte als alleiniges Einkommen bei weitem nicht zur Existenzsicherung aus, kann jedoch als willkommener Zuverdienst betrachtet werden.

415 WAGENER, Pedelle, 354 weist dies für das Jahr 1848 für Pedell Weißleder nach.

416 Vgl. WAGENER, Pedelle, 354.

417 Dies widerspricht z.B. der Aussage Jochen Brünings im Rahmen des Symposiums „Zur Geschichte der Museen im 19. Jahrhundert. 1789-1918“, Institut für Museumskunde, Staatliche Museen zu Berlin, Berlin 2./3. Dezember 2004, der postulierte, dass Führungen in Universitätssammlungen stets durch ausgewiesenes Fachpersonal, namentlich die Leiter der Sammlungen, durchgeführt worden seien. Damit begründete Brüning zudem die eingeschränkten Öffnungszeiten der Museen: Die starke universitäre Anbindung ließe den Kustoden nicht mehr Zeit dazu. Diese Argumentation trifft für Göttingen augenscheinlich nicht zu.

418 Zitiert nach WAGENER, Pedelle, 353. đ̊̄ steht für März. 
genau kennen mußte, um sachkundige Erläuterungen abgeben zu können.“419 Dies wäre immerhin ein kleiner Beleg für die öffentliche Zugänglichkeit des Museums im ausgehenden 18. Jahrhundert - die Zeit, in der noch kein Besucherbuch existierte oder aus der zumindest keines erhalten ist.

In derselben Akte, die schon die Dienstanweisung Blumenbachs enthielt (vgl. Kap. 2.2.3), findet sich auch der Entwurf der Dienstanweisung für den Hussier ${ }^{420}$ des Museums. Sie stammt aus der napoleonischen Ära, genauer vom 14. Juni 1813, weshalb als Absendeort Kassel angegeben ist.

\author{
Instruction für den Hüssier beÿ dem \\ NaturalienCabinete des academischen \\ Museum Zu Göttingen.
}

Der Staatsrath, Generaldirector des öffentlichen Unterrichtes, beschließt:

Art 1.

Die Dienstgeschäfte des beÿ dem Naturalien Gabinete des academischen Museum zu Göttingen angestellten Huissiers sind folgende:

I. Hat derselbe in dem Naturalien Museum Cabinete diejenigen Fremden oder Studierenden auf der Universität, welchen dasselbe nicht von ... dem Director ...... selbst gezeigt wird, herumzuführen. Hierbeÿ wird ihm nachdrücklichst zur Pflicht gemacht:

1. nie mehr als vier höchstens sechs Personen zu gleicher Zeit herum zu fuhren [sic];

2. sie anzuhalten, immer beÿsammen zu bleiben;

3. nie zu gestatten, daß Jemand von der Gesellschaft vor der Zeit in ein anderes Zimmer sich begebe, oder in einem, welches eben gezeigt worden ist, zurückbleibe;

4. nie zuzugeben, daß Jemand außer ihm etwas von den Naturalien oder KunstSachen anfaße, oder einen Schiebkasten ausziehe; weshalb denn auch die Glasschränke, der Regel nach, nicht geöfnet [sic] werden dürfen, zumal da die wichtigsten Gegenstände, und welche den Fremden gewöhnlich gezeigt werden, so aufgestellt sind, daß man sie auch durch die Glasscheiben bequem in Augenschein nehmen kann.

II. Muß derselbe auch dem Museum gegenwärtig seÿn, so oft der Professor, Ritter Blumenbach, den Zuhörern seiner Vorlesungen über die Naturgeschichte, oder der Professor Heeren denen, welche sein Collegium über die Ethnographie besuchen, das dahin Gehörige in dem Museum demonstriren.

${ }^{419}$ WAGENER, Pedelle, 353.

420 „Huissier ([...], v. altfranz. Huis, „Thür““), Thürsteher, Thürschließer, ursprünglich ein Hofdiener, welcher die Aufsicht bei den Thüren im Innern der königlichen Schlösser führte, jetzt ein Diener, welcher im Vorzimmer eines Ministers oder sonstigen hohen Staatsbeamten die Anmeldung und Einführung zu besorgen hat; auch Bezeichnung für die Diener parlamentarischer Körperschaften u. dgl. In der französischen Gerichtsorganisation sind die Huissiers bei den Gerichten funktionierende Beamte (officiers ministériels), welche unmittelbar von den Parteien oder den Staatsanwalten gebraucht werden, teils um Ladungen, Aufforderungen und Benachrichtigungen an die andre Partei oder Anwalte gelangen zu lassen, teils um richterliche Anordnungen zu vollstrecken. Die Huissiers werden auf Vorschlag des Justizministers vom Präsidenten der Republik ernannt. Sie bilden wie die Avoués und Notare in jedem Arrondissement eine Gemeinschaft, indem sie aus ihrer Mitte eine Disziplinarkammer wählen, deren Oberaufsicht sie unterstellt sind. Dem Institut der Huissiers ist das deutsche Institut der Gerichtsvollzieher (s.d.) nachgebildet. [...]“; Meyers KonversationsLexikon. Ein Nachschlagewerk des allgemeinen Wissens, Bd 9, 5., gänzl. neubearb. Aufl., Leipzig und Wien 1896, 35. 
III. Muß derselbe gleichfalls ... auf dem Museum sich aufhalten, so oft Handwerker darin arbeiten, und die möglichste Vorsorge anwenden, so oft gescheuert wird, die Fenster gepuzt oder ähnliche Arbeiten darin vorgenommen verrichtet werden.

IV. Hat er die gewöhnlichen Geschäfte Jahr aus Jahr ein zu besorgen, als z.B. von Zeit zu Zeit nach Motten und andern Schädlichkeiten sorgfältig zu suchen; bey trockenem ... Wetter die Fenster ...zu öfnen, um einen Luftzug zu unterhalten; das Auspacken der ankommenden Naturalien zu besorgen, beÿm Ein- und Umrangiren Hülfe zu leisten. Deshalb wird es ihm auch zur Pflicht gemacht, jeden Morgen beÿ dem Director des Musei anzufragen, ob und welche Geschäfte der Art etwa zu besorgen seÿen.

Art 2.

Der Professor und Director des Musei, Ritter Blumenbach wird beauftragt, für die Versehung des gegenwärtigen Beschlusses Sorge zu tragen. ${ }^{421}$

Hier wird noch einmal ganz deutlich festgeschrieben, dass der Wärter zum Führen der Fremden berechtigt ist und was er dabei zu beachten hat. Darüber hinaus wird deutlich herausgestellt, wer mit der Durchführung bestimmter Aufgaben betraut und wer letztlich für die ordnungsgemäße Durchführung verantwortlich ist. Doch gibt diese Quelle über weit mehr Auskunft als nur über die offensichtlichen Dienstgeschäfte des Museumsdieners - sollte diese Instruktion denn tatsächlich so umgesetzt worden sein. Zum einen wird - schon allein durch die Streichungen - der Entwurfscharakter hervorgehoben. Zum anderen ist die Art der Streichungen bezeichnend: Sie betreffen häufig die Ersetzung des Wortes Kabinett durch das Wort Museum. Hier wurde also großen Wert auf den Namen dieser Institution gelegt. Dieser Aspekt wird im Begleitschreiben zu der Instruktion noch stärker hervorgehoben. Darin heißt es:

Da jetzt unter dem Nahmen Museum nur die Sammlungen zusammengefaßt werden, über welche Ihnen, mein Herr Professor und Ritter, die Direction übertragen worden ist, so habe ich auch in der Instruction des Ausdruckes NaturalienCabinet des academischen Musei mich nicht bedient, sondern stets den Ausdruck Museum gewählt. ${ }^{422}$

Allerdings sind das die einzigen Quellenbelege, in denen die Namensgebung thematisiert wird. Darüber hinaus gibt die Dienstanweisung Auskunft über Arten der Nutzung des Museums, den Publikumsverkehr darin - oder vielmehr über die Beschränkungen, denen dieser unterlag. Deutlich wird auch, dass das Museum und seine Objekte in erster Linie zu Demonstrationszwecken im Rahmen von Lehrveranstaltungen und Führungen genutzt wurden. Hierauf wird im folgenden Kapitel näher einzugehen sein. Zunächst sollen jedoch kurz die Wärter vorgestellt werden.

${ }^{421}$ UAG Kur 4 V g 5, 22r-23r. Texthervorhebungen im Original.

422 UAG Kur 4 V g 5, 19r. 


\section{Johann Ludwig Lorenz}

Johann Ludwig Lorenz wurde ca. 1736 in Burgtonna bei Gotha geboren. Er hatte beim Regiment Sachsen-Gotha gedient, von dem er sich jedoch seinen Abschied erkaufte. Von 1767 bis 1787 war er als Polizeijäger tätig. Zusätzlich trat er etwa 1768 seinen Dienst bei Heyne an, der ihm wohl auch die Stellung als Museumswärter verschaffte. Die Tätigkeit als Museumswärter und Pedell der Societät der Wissenschaften versah er im Zeitraum nach 1776 bis in sein Todesjahr $1809 .{ }^{423}$

\section{Johann Ludolph Ernst Stiepel}

Stiepel wurde 1761 in Roringen geboren. Er hatte 21 Jahre lang beim Militär gedient. 1793 bekleidete er die Position eines Fourier ${ }^{424}$ beim 9. Infanterieregiment. Gleichzeitig war er Polizeijägersergeant. Ebenfalls 1793 vermählte er sich in Roringen mit Johanna Dorothea Elisabeth Bode. 1804 erscheint er in den Quellen als Adjunkt. Im darauf folgenden Jahr wurde er Bibliothekspedell. 1809 trat Stiepel die Nachfolge Lorenz’ als Museumswärter an. Johann Ludolph Ernst Stiepel verstarb am 25. Januar 1828.

\section{Friedrich Carl Rudolph Weißleder}

Die Nachfolge Stiepels trat der ca. 1782 in Ilfeld geborene Weißleder an. Als Sohn eines Ackerknechts verdiente er sein Geld zunächst als Bedienter und Kutscher. Nachdem er 1818 bereits zweiter Bibliothekspedell geworden war, wurde er 1828 zum ersten Bibliothekspedellen, Museumswärter und Societätspedell. Zudem arbeitete er als Wärter in der zoologischen Abteilung des Physiologischen Instituts. Weißleder, der seine Ämter bis in sein Todesjahr ausübte, verstarb 1863.

Zusätzlich zu den Museumswärtern lassen sich, allerdings erst für die Zeitraum ab 1832, Nachtwärter nachweisen. ${ }^{425} \mathrm{Zu}$ ihren Aufgaben gehörte es, in regelmäßigen

423 WAGENER, Pedelle, 353, 527.

${ }^{424}$ Ein Fourier ist „ein Unter-Officier, welcher das Commis-Brod empfänget, und selbiges austheilet. Er macht Quartier vor die Compagnie, theilet die Bilette aus, und träget in denen Guarnisonen Sorge, daß jeder Bürger seinem Soldaten Bett, Dach und Gemach, süß und sauer, Feuer und Licht gebe. Sein Gewehr ist eine Hellebarte oder Flinte.“ Angabe aus ZEDLER, Bd 9, Sp. 1586.

425 Zuvor hatten Mitglieder der Garnison den nächtlichen Wachdienst übernommen. 1833 wurde die Garnison zahlenmäßig jedoch soweit eingeschränkt, dass dafür keine Kapazitäten mehr waren. Vgl. WAGENER, Pedelle, 525. 
Abständen auf festgelegten Wegen bei Bibliothek, Museum und Concilienhaus zu patrouillieren, sich von der Unversehrtheit der Gebäude zu überzeugen, und auf verdächtige Personen ebenso Acht zu geben wie auf Brandgefahr. ${ }^{426}$ Der Lohn der Nachtwärter lag mit 24 Rthlr pro Jahr ${ }^{427}$ noch unter dem der Museumswärter, weshalb sich „,[a]uf diese Stellen [...] nur Angehörige der einkommensschwächsten Berufsgruppen [bewarben], für die selbst dieser geringe Lohn von Bedeutung war [...].“428 Zwischen 1832 und 1840 können drei Nachwärter ausgemacht werden: Georg Jürgens, angestellt von 1832 bis 1838 und danach „aus unbekannten Gründen nach Rücksprache mit der Polizeidirektion entlassen“, ${ }^{429}$ ferner Heinrich Rudolphi (von $1832 \mathrm{ab)}$ und Johann Michael Freund (von $1838 \mathrm{ab}$ ), deren Entlassungsdaten nicht bestimmt werden können. Von letzterem ist bekannt, dass er 1828 als Fabrikarbeiter und vorher als „Handarbeiter und beeidigter Holzklafterer“430 gearbeitet hatte. Lebensdaten können zu keiner dieser Personen nachgewiesen werden.

Insgesamt waren zwischen 1773 und 184017 Personen ausschließlich männlichen Geschlechts am Academischen Museum angestellt. Davon waren acht Aufseher, zwei Assistenten, einer studentische Hilfskraft und sechs Wärter im weitesten Sinne.

Das wissenschaftlich-administrative Personal der Sammlung setzte sich durchweg aus ausgewiesenen Fachwissenschaftlern zusammen. Zum Aufseher wurde offenbar nur ernannt, wer in der Universitätshierarchie zumindest die Stufe eines Privatdozenten erreicht hatte. Für eine Assistentenstelle schien eine Promotion ausreichend.

Das Aufseheramt war wohl mit einem gewissen Prestigegewinn, nicht aber mit einem direkten wirtschaftlichen Nutzen verbunden: Sowohl Blumenbach als auch Osiander und Hausmann übten das Unteraufseheramt unentgeltlich aus. Welcher Akteur welche konkreten Interessen damit verfolgte, dass er ein solches Amt antrat, ${ }^{431}$ lässt sich anhand der erhaltenen Quellen nicht mehr erschließen. Wie sich die einzelnen Ämter - Oberaufseher, Mitaufseher, Unteraufseher, Assistent - in ihren Aufgabenbe-

${ }^{426}$ Bei WAGENER, Pedelle, 525f, ist eine die Aufgaben der Nachtwärter sehr detailliert schildernde Instruktion aus dem Jahr 1838 abgedruckt.

427 Vgl. WAGENER, Pedelle, 526.

428 WAGENER, Pedelle, 525f.

${ }^{429}$ WAGENER, Pedelle, 526.

430 WAGENER, Pedelle, 526.

431 Zu denken ist hier bspw. an freien Zugang zur Sammlung für wissenschaftliche Zwecke. 
reichen unterschieden, lässt sich anhand der Akten ebenfalls nicht ermitteln. Relativ eindeutig ging es hierbei um Hierarchieebenen und Verantwortlichkeiten gegenüber der Landesregierung. ${ }^{432}$

Die Bedeutung der Mit- und Unteraufseher aus der Zeit nach 1815 wurde von der übermächtigen Reputation Blumenbachs überlagert, die er um die Jahrhundertwende erlangt hatte und der er selbst im hohen Alter nicht mehr gerecht zu werden vermochte. ${ }^{433}$ Dies führte in Bezug auf die Entwicklungen der Sammlungen des Museums zu Stagnationsprozessen.

\subsection{Die Besucher}

Nachdem im vorigen Kapitel ausführlich auf das Personal des Academischen Museums eingegangen worden ist, stehen hier nun die Besucher des Museums im Mittelpunkt. Eine Annäherung an diese Gruppe von Akteuren erfolgt über das aus dem Zeitraum 1808 bis 1837 erhaltene Besucherbuch des Museums. Die Einträge darin wurden in einer eigens für diese Magisterarbeit entwickelten Datenbank erfasst und können nun nach verschiedenen Kriterien ausgewertet werden. An dieser Stelle geht es darum, auf die Problematik mit dem Umgang des Besucherbuches als Quelle und auf die verschiedenen Möglichkeiten der Auswertung hinzuweisen. Einige exmplarische Auswertungen finden sich in Anhang B dieser Arbeit; in Kap. 5.2.2 werden exemplarische Auswertungsergebnisse diskutiert.

\subsubsection{Quellenkritik, Normalisierung der Einträge}

Bei dem Besucherbuch handelt es sich um ein heute im Bestand der Völkerkundlichen Sammlung der Georg-August-Universität Göttingen befindliches, rund 288 Seiten starkes, nicht paginiertes, Buch mit festem, schmucklosen und unbeschriftetem braunen Pappeinband. 220 Seiten sind mit Besuchereinträgen beschriftet, in der Re-

432 So finden sich im Aktenbündel UAG Kur 4 V g 20 teilweise noch aus dem Jahr 1838 Rechnungen, die nicht mehr von Blumenbachs - inzwischen sehr zittriger - Hand stammen, jedoch von ihm als Oberaufseher - unterzeichnet sind.

433 Ein sprechendes Bild dieser Situation liefert folgender Ausschnitt eines Schreibens von Hausmann an einen nicht näher spezifizierten Geheimen Kabinettsrat vom 24. Juli 1838, in dem es heißt: „Herr Obermedizinalrath Blumenbach, dessen Altersschwäche sehr zunimmt, hatte meine Bitte, die bei ihm etwa eingehenden Rechnungen für das Museum mir zur Aufstellung der allgemeinen Rechnung zu geben vergessen und, wie ich erst gestern in Erfahrung gebracht, zwei Rechnungen vom Pedell Weißleder an die Universitäts-Casse gesandt, von welcher die Zahlungsanweisung eingeholt und der Betrag bereits übermacht worden." UAG Kur 4 V g 20. 
gel mit schwarz-brauner Tinte, in einigen seltenen Fällen finden sich einzelne Einträge, die mit Bleistift vorgenommen wurden. Die Einträge stammen von verschiedenen Händen. Es ist also davon auszugehen, dass sich die Besucher - zumindest in der Regel - eigenhändig eingetragen haben. Einen Eindruck davon vermittelt die unten stehende Abbildung.

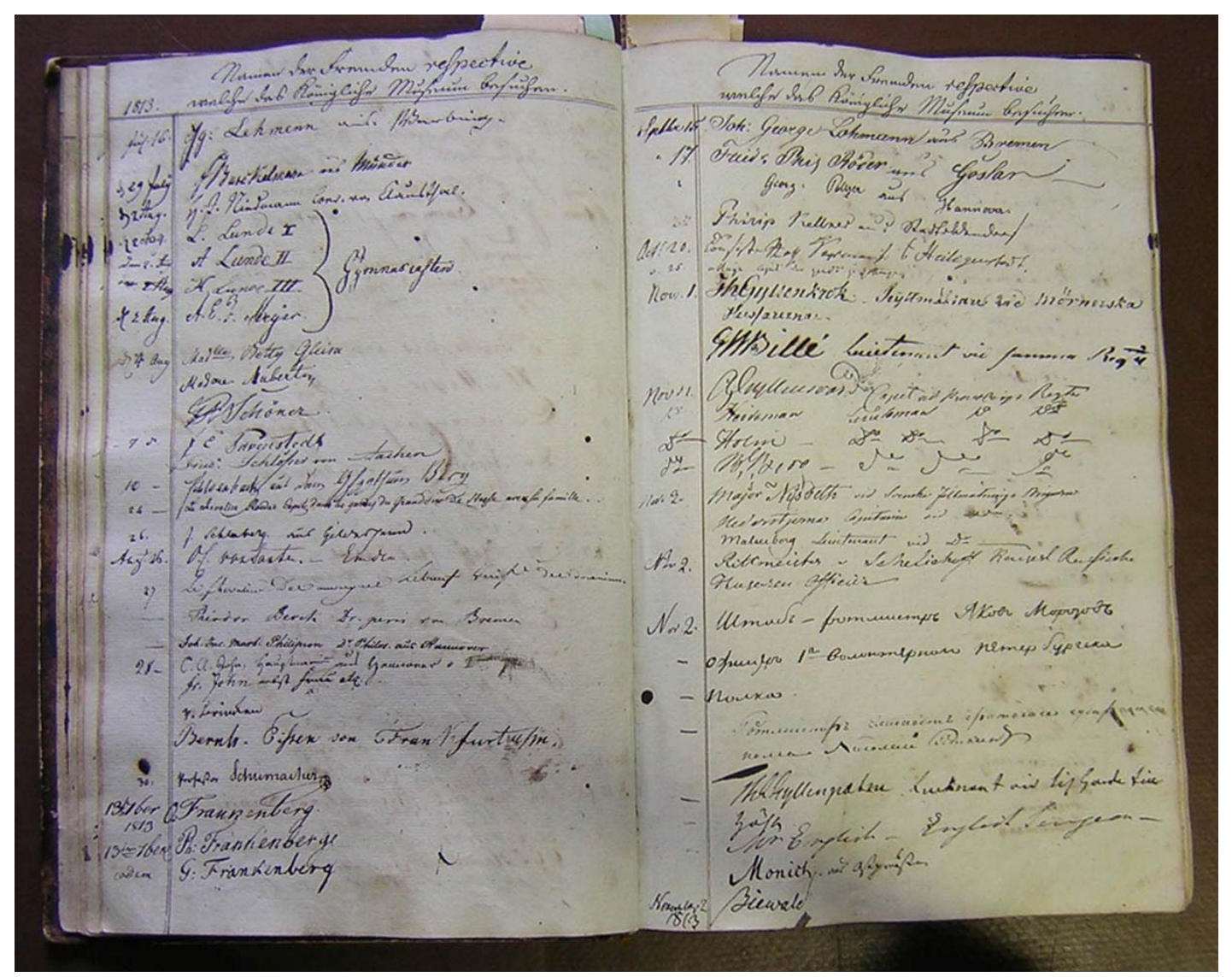

Abbildung 6: Besucherbuch des Academischen Museums, Einträge vom 16. Juli 1813 bis zum 2. November 1813

Das Buch enthält keinerlei Hinweise auf auf seinen Entstehungszusammenhang oder auf vorangegangene oder nachfolgende Besucherbücher. ${ }^{434}$ Es ist es außerordentlich schlicht gehalten und verfügt weder über irgendeinen Kommentar noch über eine Einleitung oder dergleichen. Das legt zum einen nahe, dass es sich um einen alltäglichen Gebrauchsgegenstand handelte, und zum anderen, dass es möglicherweise der Folgeband eines nicht mehr vorhandenen Vorgängerexemplars ist. Dies würde den Beginn der westphälischen Herrschaft über Göttingen als Entstehungskontext aus-

${ }^{434}$ Auch den übrigen Schriftquellen zum Academischen Museum sind hierzu keine Aussagen zu entnehmen. 
schließen. Dagegen spricht auch, dass das Buch über die napoleonische Ära hinaus weitergeführt wurde.

Ungeklärt ist, wem die Aufsicht über das Buch oblag oder von wem es verwahrt wurde. Wurde es am Eingang des Museumsgebäudes ausgelegt, so dass sich dort auch Besucher der Gemäldesammlung eintrugen - oder bei den eigentlichen Museumssammlungen? Am plausibelsten erscheint die These, dass das Buch dem Museumswärter oder einem der Aufseher anvertraut war und hervorgeholt wurde, wenn Besucher in das Museum eingelassen wurden.

Anhand der Einträge im Buch ist nicht eindeutig zu klären, ob es sich tatsächlich um ein Besucherbuch handelte, in das sich alle Besucher des Museums eintrugen, oder um ein Fremdenbuch im engeren Sinn, d.h. um ein Buch, in das sich nur Personen eintrugen, die nicht aus Göttingen kamen oder dort kein Bürgerrecht besaßen. Dazu sind zu viele Einträge enthalten, bei denen eine Ortsangabe vollständig fehlt. ${ }^{435}$ Plausibel erscheint, dass es sich vorrangig um ein Fremdenbuch im engeren Sinne handelte und dass sich Göttinger nur dann eintrugen, wenn sie von auswärtigem Besuch begleitet wurden.

Diesen ungeklärten Fragen zum Trotz liegt mit dem Besucherbuch im Grunde eine serielle Verwaltungsquelle vor, deren Einträge allerdings weit von der Einheitlichkeit entfernt sind, die man sich für eine statistische Auswertung wünscht. Für die vorzunehmenden Auswertungen musste die Struktur der Daten zunächst vereinheitlicht werden. Aufgrund des eingeschränkten zeitlichen Rahmens der für eine Magisterarbeit zur Verfügung steht, erstreckt sich die nachfolgende Auswertung nicht auf das gesamte Besucherbuch. Von der ersten Hälfte des Besucherbuches - den Zeitraum Juli 1808 bis Dezember 1820 umfassend - wurde eine Transkription angefertigt, die die Basis für die Auswertungen liefert; die zweite Hälfte wurde lediglich durchgesehen. In Bezug auf die Transkriptionen besteht insofern eine hohe Fehleranfälligkeit als nahezu jede Zeile in anderer Schrift ausgeführt ist und sich dadurch, dass sich Buchstaben i.d.R. nicht wiederholen, keine Vergleichsmöglichkeiten bieten. Darüber hinaus mussten, um die Quelle auswertbar zu machen, verschiedentlich Angleichungen vorgenommen und Kategorisierungen durchgeführt werden. In Zusammenarbeit

435 Eine Möglichkeit wäre, die Nichtangabe eines Herkunftsortes mit Göttingen als Herkunftsort gleichzusetzen. Dies ist jedoch zu spekulativ um hier als Arbeitsgrundlage dienen zu können. 
mit Arnd Beyer entstand eine Datenbank, die dies leisten kann und mit der darüber hinaus diverse Auswertungsmöglichkeiten für die Quelle eröffnet wurden. ${ }^{436}$

Bei der Normalisierung der Einträge traten etliche Hindernisse auf. Während der Abgleich der Ortsbezeichnungen, ${ }^{437}$ hier vor allem die Decodierung lateinischer Bezeichnungen und v.a. polnische Ortsbezeichnungen ehemals deutscher Städte, vergleichsweise problemlos lief, erwies sich die Zuordnung von Berufen zu Berufgruppen als erheblich diffiziler. ${ }^{438}$ Dies wurde dadurch erschwert, dass sich im Laufe des Untersuchungszeitraumes die territoriale Zugehörigkeit Göttingens veränderte und damit auch Verwaltungsstrukturen und die Berufsbezeichnungen. In einigen Fällen konnte kein Berufseintrag vorgenommen werden, da aus dem Eintrag nicht klar hervorging, ob es sich um einen Titel oder eine Berufsbezeichnung handelt. Ein gutes Beispiel hierfür ist die Bezeichnung Hofrat, die verschiedenes bedeuten konnte:

Hofräte hießen seit dem 16. Jahrhundert die Mitglieder der höchsten Kollegialbehörden, die nach dem Muster des Reichshofrats in Wien zur Erledigung von Regierungs- und Verwaltungsahngelegenheiten und als Landesgerichte höchster Instanz bestanden. Im 19. Jahrhundert (und bis 1918) wurde Hofrat als Ehrentitel in verschiedenen Stufen (Geheimer Hofrat usw.) an höhere Beamte, Professoren, Ärzte, in Preußen auch an Kanzleivorstände und verdiente Subalternbeamte verliehen. ${ }^{439}$

\subsubsection{Exemplarische Auswertungsergebnisse}

Nach der Angleichung der Datensätze lassen sich für den Zeitraum 5. Juli bis 18. März 1821 mindestens 3099 Besucher in 2926 Einträgen nachweisen. Mindestens deshalb, da sich verschiedentlich Personen mit dem Vermerk „mit Familie“ eingetragen haben und mangels weiterführender Informationen die Wahl zwei als Mindestangabe zugrunde gelegt wurde. Dies betrifft 21 Einträge. Bei den Einträgen „Mit Gesellschaft“ (vier Einträge) und „Mit Kindern“ bzw. „Zöglinge“ (zwei Einträge) wurden jeweils 3 Besucher angenommen.

436 Eine ausführliche Anleitung zum Umgang mit der Datenbank, insbesondere zum Erstellen von Auswertungen, ist auf der beiliegenden CD enthalten.

437 Hier unverzichtbar: Helmut PLECHL (Hg.), Orbis Latinus, Lexikon lateinischer geographischer Namen des Mittelalters und der Neuzeit, Großausgabe, Braunschweig 1972.

438 Hilfreich: KLAUS-JOACHIM LORENZEN-SCHMIDT, Lexikon historischer Berufe in SchleswigHolstein und Hamburg, Kiel 1996.

439 Rudi PALlA, Verschwundene Arbeit. Ein Thesaurus der untergegangenen Berufe, Frankfurt a.M. 1995. 
Exemplarisch ausgewertet wurden die Verteilung der Besucher nach Geschlecht und nach Entfernung zum Herkunftsort sowie die Fluktuation des Besucherstromes über den Gesamtzeitraum.

Untersucht man die Verteilung der Besucher nach Geschlecht, so fällt auf, dass der Frauenanteil für den Gesamtzeitraum in den Monaten Juli und August nahezu doppelt so hoch ist, wie in den übrigen Monaten. Auch bei der Auswertung nach Jahren gibt es Auffälligkeiten: In den Jahren 1811 (10,51\%), 1816 (9,66) und im Eröffnungsjahr des Besucherbuches 1808 (18,44) liegt der Frauenanteil erheblich über dem der restlichen Jahre mit 5,73\%. Für das Jahr 1808 lässt sich der Anteil dadurch erklären, dass die ersten Einträge aus dem Juli des Jahres stammen und damit die Sommermonate, in denen der Frauenanteil höher ist, ein stärkeres Gewicht im Jahresdurchschnitt bekommen. Außerdem fällt auf, dass bei den EntfernungsHistogrammen die Entfernung von 180 bis $210 \mathrm{~km}$ mit einem Frauenanteil von 12,18\% hervorsticht, während der Durchschnitt der restlichen Entfernungen bei 6,17\% liegt. In genau diesem Entfernungsbereich liegen die Städte Bremen und Frankfurt a.M., die mit 94 von 156 Besuchern aus diesem Entfernungsbereich maßgebend für die Verteilung sind. Der Frauenanteil aus diesen beiden Städten allein beträgt 13,8\%. Dies legt die Vermutung nahe, dass der Frauenanteil bei Besuchern aus Großstädten höher ist als bei den anderen Besuchern. Bei genauerer Überprüfung stellt man jedoch fest, dass dies zwar für einige Städte (Bremen: 20\%, Hamburg: 11\%) aber nicht für alle (Berlin: 2\%) gilt.

Eine Entfernungsanalyse aller Besucher zeigt zum einen, dass ein Großteil der 2491 Besucher, bei denen Ortsangaben zur Verfügung standen, aus Orten kamen die bis zu $120 \mathrm{~km}$ von Göttingen entfernt liegen. Bei größeren Entfernungen sinkt die Besucherzahl deutlich, und nimmt nur bei den kumulierten Entfernungen über $270 \mathrm{~km} \mathrm{zu}$. Das dieser Bereich so dominant ist liegt vermutlich hauptsächlich an der größeren Menge der Orte. Es zeigt aber auch, dass das Museum auch für Besucher aus weit entfernten Orten von Interesse war. Im Zusammenhang mit den Entfernungen sind natürlich auch die Orte interessant, aus denen die meisten Besucher kamen, was mit 72 Einträgen Göttingen selbst ist, gefolgt von Münden (63 Einträge, 23km), Hamburg (62 Einträge, 227km), Bremen (60 Einträge, 189km) und Berlin (49 Einträge, 256km). Um genauere Aussagen über die Herkunft der Besucher zu erhalten, reicht 
die einfache, geographische Entfernungsanalyse nicht aus. Hier müsste man den 679 Orten weitere Kategorisierungsparameter wie Handelsstadt oder Universitätsstadt hinzuzufügen, was in Ansätzen bereits in der Datenbank realisiert wurde. Zudem wäre es zu überdenken, mit symbolischen Kategorien zu operieren, also z.B. Kassel für die Zeit der Westphälischen Herrschaft eine größere symbolische Nähe zuzuschreiben, als dies unter hannöverischer Herrschaft der Fall gewesen wäre. Im Anschluss daran müsste anhand des Besucherbuches geprüft werden, ob diese Argumentation haltbar ist.

Insgesamt fluktuiert der Besucherstrom stark. Betrachtet man die zugehörige Auswertung, ${ }^{440}$ so fällt auf, dass die für die Jahre 1809-1820 durchschnittlich angenommene Besucherzahl von 245 in den Jahren 1809/1810 und 1812/1813 erheblich unterschritten, hingegen in den Jahren 1811, 1814 und 1820 erheblich überschritten wird. Betrachtet man die einzelnen Einträge der zugehörigen Zeiträume, so lässt sich zumindest für das Jahr 1814 der hohe Besucherstrom mit dem Durchzug von Militärbataillonen erklären. In den anderen Fällen liegen keine ebenso offensichtlichen Erklärungen vor.

Innerhalb der einzelnen Jahre zeichnet sich ein Besucherhoch in den Sommermonaten, vor allem im August ab, während der Besucherstrom in den Wintermonaten teilweise auf null zurückgeht. Eine Abweichung von diesem Muster gibt es vor allem für das Jahr 1815, in dem der zweitgrößte Besucheranstrom im Monat November verzeichnet wird. Hier lässt sich wiederum ein hoher Anteil an Militärangehörigen feststellen. Ansonsten lassen sich die saisonalen Schwankungen in weiten Teilen durch Witterungsbedingungen erklären. Zum einen waren die Räume des Academischen Museums selbst nicht heizbar, so dass ein Besuch im Winter vorstellbar unattraktiv war. ${ }^{441}$ Entscheidender war, dass im Winter aufgrund der Wetterbedingungen, aufgeweichte oder durch Schnee versperrte Wege nur dann lange Reisen unternommen wurden, wenn es unvermeidlich war. Hingegen gelten die Sommersemesterfe-

${ }^{440}$ Vgl. Anhang B: Auswertung des Besucherbuchs.

${ }^{441}$ Vgl. z. B. UAG Kur 4 V g 46, 68v, 69r. Die Unmöglichkeit die Räume zu heizen hielt nicht nur Besucher fern, sondern sorgte auch dafür, dass die Arbeiten an den Beständen nicht fortgesetzt werden konnten. So heißt z. B. an bezeichneter Stelle bezüglich des Umgangs mit dem Blumenbach-Nachlass: ,[D]a die Räume des Akademischen Museums nicht heizbar sind, so wird die Aussonderung, Säuberung und Einordnung der in den Blumenbach'schen Sammlungen enthaltenen Gegenstände sich erst im nächsten Sommer bewerkstelligen lassen.“ 
rien traditionell als Zeitraum für kürzere Gelehrtenreisen und Reisetätigkeit insgesamt. Dies lässt sich anhand der verfügbaren Daten allerdings nicht explizit belegen. ${ }^{442}$ Matuschek, der jüngst eine Auswertung des Besucherbuchs des Herzog Anton Ulrich-Museums in Braunschweig vornahm, ${ }^{443}$ verweist zudem auf die Relevanz von Messen, als Ereignisse, die Publikum in die Stadt und damit potentielle Besucher ins Museum lockten. Hier ist vor allem an Kaufleute zu denken. Ob sich ähnliches auch für Göttingen nachweisen lässt, bleibt zu prüfen.

Generell stellt sich die weiterführende Frage, was eigentlich ein Museumsbesuch ist. Kann bereits der reine Besuch des Museums als wissenschaftliche Praxis gelten? Welche Form der sozialen Praxis stellt ein solcher Besuch dar und in welchem Zusammenhang erfolgte er? Angenommen, ein Reisender hat drei Tage Aufenthalt im Göttingen - was sieht er sich an? Bereits Zedler berichtete, dass Kabinette „von Durchreisenden mit Lust besuchet“ würden. ${ }^{444}$ Das lässt den Rückschluss auf einen gewissen Sammlungstourismus zu, auch wenn damit nicht gesagt ist, dass der Besuch der Sammlungen der Zweck der Reisen war. Gehörte das Academische Museum also in einen festen Besuchskanon? Wenn ja: Für wen galt er und was gehörte noch dazu? Hier können für akademische Kreise vor allem Gelehrtenbesuche, der Besuch der Akademie der Wissenschaften und auch der Besuch des Botanischen Gartens angenommen werden. Ein ausführliches Eingehen auf diesen Themenkomplex würde den Rahmen der vorliegenden Arbeit sprengen, wäre aber eine interessante Anregung für eine weiterführende Untersuchung.

Im Zusammenhang der Besuchspraxis scheint die Frage interessant, ob die Besucher allein oder in Gruppen kamen. Unter der Vorannahme, dass man alle Personen, die aus einem Ort kommen, und die am selben Tag das Museum besuchen als Gruppen wertet, erhält man folgendes Ergebnis: Es sind 443 Gruppen mit 1178 Besuchern, davon 26 Gruppen mit insgesamt 66 Besuchern ohne Ortsangabe. Als Gruppe sind dabei aber auch Familien, Männer mit Ehefrauen und Kindern berücksichtigt. Wertet man Gruppen nur so, dass sie auch aus mehreren Einträgen stammen müssen bleiben 357 Gruppen mit 945 Besuchern.

${ }^{442}$ Mittelt man die Zahl der Akademiker über den Gesamtzeitraum und stellt ein prozentuales Verhältnis zur Gesamtbesucherzahl her, so ergeben sich hier keine signifikanten Werte.

443 Vgl. MATUSCHEK, Besucher und Besucherbetreuung, bes. 88f.

${ }^{444}$ Vgl. Kap. 2.2.5 der vorliegenden Arbeit bzw. Zedler, Bd 15, Sp. $2143 f$. 
Die Antwort auf die Frage, wie viele Besucher das Museum mehrfach besuchten ist mit einer hohen Fehleranfälligkeit behaftet. Dies liegt vor allem an Namensdopplungen (die häufig durch fehlende Zusatzangaben wie z.B. Vornamen) auftreten, aber auch an unterschiedlichen Schreibweisen des gleichen Namens etc. Wählt man als Kriterien, dass die Einträge von verschiedenen Tagen stammen müssen und dass die einträge ferner gleiche Nachnamen, gleiche Vornamensbuchstaben und gleiche Ortsangaben aufweisen sollen, so kann man mit hoher Wahrscheinlichkeit 22 wiederkehrende Besucher (siehe Anhang B) bestimmen - was sich gegenüber eines Gesamtbesucheraufkommens von über 3000 Personen allerdings sehr gering ausnimmt.

Von hohem Interesse ist die Frage, ob sich von Seiten der Besucher Stellungnahmen zu den Besuchern finden lassen. Allerdings verursacht ihre Beantwortung einen sehr hohen zeitlichen Aufwand, der soweit die Recherchen gediehen sind, durch die Ergebnisse nicht gerechtfertigt wird. Bei der Masse an Besuchern muss eine Überprüfung darüber, ob es Rückmeldungen über das Museum oder überhaupt eine Bestätigung für den Besuches gab, notwendigerweise stichprobenartig bleiben. Im Folgenden findet sich eine kurze Auflistung prominenter Persönlichkeiten, zu denen eine lebensweltliche Kontextualisierung möglich war. In einem nächsten Schritt, zu Teilen ist dies schon ergebnislos geschehen, müssten ihre Publikationen und Selbstzeugnisse systematisch nach betreffenden Aufzeichnungen durchgesehen werden.

Johannes Amsinck aus Hamburg (1792-1879)

Besuch des Museums am 4. Juli 1814

Stammte aus einer Hamburgischen Kaufmannsdynastie. Die Amsinck Comp. war an der Überführung der Sammlungsstücke der Cook-Sammlung beteiligt.

Friedrich Ludwig Freiherr von Berlepsch (1749-1818)

Besuch des Museums am: 8. November 1813

Jurist, Hannoverscher Staatsmann, Alumnus der Georgia Augusta.

Johann Baptist Alexius Graf Coronini-Cronberg (1794-1880)

Besuch des Museums am: 19. Juni 1811

Österreichischer Armee-Kommandant.

Georges Léopold Chrétien Frédéric Dagobert Baron de Cuvier (1769-1832)

Besuch des Museums am: 5. August 1811

Frz. Naturforscher, Anatom, Mitbegründer der wissenschaftlichen Paläontologie, Bedeutender Vertreter des Dogmas der Konstanz der Arten und der Katastrophentheorie. 
Wilhelm (Tell) von Fellenberg (1798-1880)

Besuch des Museums am: 28. August 1820

Sohn eines Pädagogen, erzogen von Therese Forster;

Naturkundliches interessiert. 1820/21 zu einem Studienaufenthalt in Deutschland.

Therese Forster (1786-1862)

Besuch des Museums am: 10. Mai 1811

Tochter Georg Forsters und Therese Heynes;

Wohl im Rahmen eines Familienbesuchs in Göttingen.

Betty Gleim (1781-1827)

Besuch des Museums am: 4. August 1813

Pädagogin und Schriftstellerin.

Eduard Gmelin (1786-1873)

Besuch des Museums am: 5. August 1816

Jurist aus Stuttgart; Älterer Bruder des Chemikers Leopold Gmelin.

Fürst Karl August von Hardenberg (1750-1822)

Besuch des Museums am: 18. September 1820

Königl. Preuss. Staatskanzler; Alumnus der Georgia Augusta.

Theodor von Humboldt-Dachroeden (1797-1871)

Besuch des Museums am: 8. Oktober 1814

Sohn (3. Kind) von Caroline von Dachroeden und Wilhelm von Humboldt.

Dorotheus Ludwig Christoph Graf von Keller (1757-1827)

Besuch des Museums am: 18. August 1809

Regierungspräsident, preußischer Staatsminister, laut Besucherbuch „Mitglied der Westphälischen Reichsstände“.

Karl Friedrich Heinrich Marx (1796-1877)

Besuch des Museums am: 26. April 1819

Arzt Biograph und Blumenbachs.

Blasisus Merrem (1761-1824)

Besuch des Museums am: 16. April 1817

Schlüler Blumenbachs, Professor der Zoologie in Marburg;

Hinterließ dem Museum ausgestopfte Tiere.

Philipp Friedrich David Murray (1770-1828),

Besuch des Museums am: 24. April 1814

Apotheker in Göttingen, Zulieferer des Museums.

Lorenz Oken (1779-1851)

Besuch des Museums am: 6. Oktober 1810

Naturforscher, Naturphilosoph; Habilitierte sich 1805 in Göttingen, in diesem Jahr auch Privatdozent an der Georgia Augusta, Herausgeber der Zeitschrift Isis. 


\section{Christian Friedrich Osiander (1789-1839)}

Besuch des Museums am 3. Oktober 1812

(1789-1813) württembergischer Buchhändler, Sohn des Gynäkologen Friedrich Benjamin Osiander, und damit Bruder Johann Friedrich Osianders, der seit 1812 am Academischen Museum tätig war.

Dorothea Rodde (1770-1825)

Besuch des Museums am 18. Juni 1810

Promovierte Tochter des Göttinger Professors für Staatsrecht und Geschichte August Ludwig Schlözer. Bekannt als „Madame Doctorin Schlözer“.

August Wilhelm von Schlegel (1767-1845)

Besuch des Museums am: 4. November 1813

Bruder Friedrich von Schlegels, 1796-1803 verheiratet mit Caroline Michaelis, der Tochter des Göttinger Orientalisten Johann David Michaelis.

\section{Stelzner}

Besuch des Museums am: 1. März 1814

Wohl ein Sohn des Oberbergmeister Georg August Stelzer aus Clausthal, der Vorbesitzer eines Teils der mineralogischen Sammlung des Academischen Museums war.

\section{de Marees van Swinderen}

Besuch des Museums am: 15. July 1819

[Keine weiteren Angaben gefunden.]

\section{Wilhelm Gottlieb Tilesius von Tilenau (1769-1857)}

Besuch des Museums am: 27. April 1815 und am 15. Juli 1817

russischer Naturforscher, Arzt, Zeichner, Kupferstecher, begleitete u.a. Krusenstern auf seiner Erdumsegelung (1803-1806);

Lebte zeitweilig in Göttingen.

Maximilian Prinz zu Wied Neuwied (1782-1867)

Besuch des Museums am: 2. May 1811

Schüler Blumenbachs, immatrikuliert an der Georgia Augusta am 16. April 1811.

Zu den wenigen Personen, die sich zu ihrem Museumsbesuch geäußert haben gehört

Fürst von Hardenberg. In seinen autobiographischen Aufzeichnungen heißt es:

„<Mo> 18 Nach Göttingen - Auf der Bibliothec - Die Professoren Reuhs, Beneke, Himly - Das Naturalien Cabinet - Die Mahlereyen - Das neue Observatorium vor dem Geismar Thore - Prof[essor] ${ }^{445}$. Der botanische Garten - Prof[essor] Schrader - Die Reitbahn. Zurück nach Hardenberg. “446

${ }^{445}$ Klammer in der Vorlage; für den nachfolgenden Namen ist eine Lücke gelassen; CN.

446 Karl August von Hardenberg/Thomas Stamm-Kuhlmann (Hg.), Karl August von Hardenberg 1750 - 1822. Tagebücher und autobiographische Aufzeichnungen, München 2000, 895. 
Zwar enthält diese knappe Aufzählung keine weiterführenden Aussagen zum Museum, aber sie bettet den Besuch in einen Kontext ein - möglicherweise ein typisches Programm für den oben erwähnten Besucherkanon.

An dieser Stelle schließt sich die Frage an, ob sich ein Zusammenhang zwischen den Besuchern erstellen lässt, oder ob und wie sich (wissenschaftliche) Netzwerke im Besucherbuch des Museums abbilden. Dies soll im Folgenden anhand eines Beispiels exemplifiziert werden: Für den 28. August 1820 findet sich der Eintrag „W von Fellenberg aus Hofwyl. in der Schweiz." Sehr wahrscheinlich handelt es sich dabei um Wilhelm von Fellenberg, einen Sohn Philipp Emanuel von Fellenbergs und Margarethe Tscharners, und damit um den Sohn einer Berner Patrizierfamilie. ${ }^{447}$

\footnotetext{
„Das Ehepaar [Emanuel und Margarethe Fellenberg] gründete im Verlauf von vierzig Jahren verschiedene Schulen, in denen Kinder aus allen Ständen in einer familiären Umgebung ihre Ausbildung erhalten sollten. Die unter dem Namen Hofwyl zusammengefassten Bildungsanstalten wurden als Familienunternehmen geführt, in dem auch die eigenen Kinder Aufgaben und Pflichten in der Verwaltung und im Unterricht zu nehmen hatten.“448
}

Eines der Kinder, genauer der erste Pflegesohn, der 1806 von Fellenbergs aufgenommen wurde, war Aimé Huber, ${ }^{449}$ ein Sohn Therese Hubers, ${ }^{450}$ die „von den pädagogischen Idealen Fellenbergs angetan“ war und sich ihrerseits um pädagogisches Personal für die Fellenbergs bemühte. ${ }^{451}$ Zudem wollte Emanuel Fellenberg „die Gefälligkeiten, welche Therese Hubers Vater, der Altphilologe Christian Gottlob Heyne (1729-1812), seinem Vater während dessen Studienzeit in Göttingen ein halbes Jahrhundert zuvor erwiesen hatte, vergelten.“ ${ }^{452}$ Darüber hinaus war Therese Forster, ${ }^{453}$ die Tochter Therese Heyne-Forster-Hubers und Georg Forsters von Januar 1808 bis Juli 1809 in Hofwyl als Erzieherin tätig. Sie „betrieb gemeinsame Lektüre

447 Diese Analyse war nur möglich mit Hilfe der 2002 erschienenen Dissertation Wittwer Hesses, die sich ausführlich mit der Familiengeschichte der Fellenbergs und den Erziehungsidealen und methoden auseinandersetzt. Vgl. Denise WiTTwer Hesse, Die Familie von Fellenberg und die Schulen von Hofwyl. Erziehungsideale, „Häusliches Glück“ und Unternehmertum einer Bernischen Patrizierfamilie in der ersten Hälfte des 19. Jahrhunderts. Den Hinweis auf diese Dissertation verdanke ich Herrn Wiard Hinrichs, Göttingen.

448 WiTTwER Hesse, Die Familie von Fellenberg, 11.

449 Victor Aimé Huber (1800-1869), achtes Kind von Therese Huber.

450 Zitate aus WiTTwer Hesse, Die Familie von Fellenberg, 93; Therese Huber, geb. Heyne, verw. Forster (1764-1829).

451 WiTTWER Hesse, Die Familie von Fellenberg, 67.

452 WitTWER Hesse, Die Familie von Fellenberg, 67f.

453 Eigentlich: Maria Theresia Forster (1786-1862). Leider fand sich zu Therese Forster nur die - wie schon Wittwer Hesse bemerkt - „leider nicht sehr ergiebige Arbeit“ Kantzenbachs. Vgl. WITTWER Hesse, Die Familie von Fellenberg, 275; und FRIEDRICH Wilhelm KANTZENBACH, Georg Forsters Tochter. Therese, die blonde Polin, Saarbrücken-Scheidt 1989. 
mit den Kindern der Familie v. Fellenberg und ihrem Bruder Aimé und unterrichtete sie in Geschichte, Geographie und Rechnen.““54 1820/21 befand sich Wilhelm von Fellenberg zu einem Studienaufenthalt in Deutschland, augenscheinlich vor allem in Berlin. ${ }^{455}$ Über einen Aufenthalt in Göttingen berichtet Wittwer Hesse allerdings erst für das Jahr 1828, so dass man den genauen Grund für den Aufenthalt 1820 nicht kennt. Möglicherweise handelt es sich lediglich um eine Zwischenetappe auf dem Weg nach Berlin, jedenfalls aber wird er Göttingen und wohl auch dem Academischen Museum nicht unvoreingenommen gegenübergestanden haben.

Der Lebensweg Therese Heyne-Forster-Hubers lässt vermuten, dass die weiblichen Mitglieder der Göttinger Professorenfamilien untereinander ebenso komplexe, wenn auch anders strukturierte Netzwerke ausbildeten, als die in dieser Untersuchung im Brennpunkt stehenden Wissenschaftler. Dem wäre weiter nachzugehen. Zumindest gibt es zwischen einigen Professorentöchtern Briefwechsel.

\subsection{Zugänglichkeit und Nutzung des Academischen Museums.}

Aussagen über die Zugänglichkeit des Museums zu treffen gestaltet sich verhältnismäßig schwierig. Betrachtet man die in Kapitel 2.2.3 analysierte Dienstanweisung Blumenbachs aus dem Jahr 1776, so geht daraus eindeutig hervor, dass das Museum darauf ausgelegt war, Besuch zu empfangen. Allerdings erfährt man aus den Verwaltungsakten des Universitätsarchivs nichts über Öffnungszeiten, Zugangsmodalitäten, Preise und dergleichen mehr. Erst für den Zeitraum nach 1837, als das Museum einer breiteren Öffentlichkeit zugänglich gemacht werden sollte, findet sich eine Ausarbeitung dazu in den Kuratorialakten.

Doch was ist mit den 64 Jahren davor? Bereits aus Kapitel 2.1 ist bekannt, dass sowohl fürstliche Sammlungen als auch späterhin bürgerliche Sammlungen zeit ihres Bestehens zu bestimmten Anlässen ausgewählten Besuchergruppen offen standen. Weiterhin ist bekannt, dass um 1700 zunehmend auch bürgerliche Sammlungen entstanden, in denen der Aspekt des Lernens aus der Natur denjenigen der Repräsentation überstieg, und dass von der Mitte des 18. Jahrhunderts an diese und auch die fürstlichen Sammlungen zunehmend einem größeren Publikum geöffnet wurden.

\footnotetext{
${ }^{454}$ WitTwer Hesse, Die Familie von Fellenberg, 93.

455 Vgl. WitTwer Hesse, Die Familie von Fellenberg, 185.
} 
Wie gezeigt worden ist, passt sich das Academische Museum in vielerlei Hinsicht dem zeittypischen Trend an. Es liegt also nahe, dies auch für den Aspekt der Zugänglichkeit anzunehmen. Doch welche Belege lassen sich dafür anführen? Betrachtet man die Verwaltungsquellen, so weist vor allem Punkt fünf der Dienstanweisung Blumenbachs das Museum eindeutig als für Liebhaber, Fremde und Studierende geöffnet aus:

Ihm lieget das Herumführen der Fremden und der hiesigen Liebhaber vorzüglich ob, und so, wie sich der Hofrath Heyne, so ofte sich Fremde an ihn wenden, ihn, den Unteraufseher ersuchen wird, zugegen zu seyn, so wird er hinwiederum den Hofrath Heyne von der Gegenwart derer benachrichtigen, die sich bey ihm melden werden, um herumgeführet zu seyn. Insonderheit gilt dieß bey den hier studierenden, die das Kabineet besehen wollen, denen willfährig zu seyn, sich ohnedem leicht eine Zeit voraus verbreden läßt. Er wird auch hirbey alle Vorsicht brauchen, daß keine zu grose Anzahl, auch nicht allerhand Personen ohne Auswahl, in das Kabinet eingelassen werden; zumal so lang die Sachen noch nicht unter den gehörigen Beschluß gebracht sind. ${ }^{456}$

Diese Praxis wurde im Jahre 1813 - allerdings mit dem Unterschied, dass die Aufgabe des Herumführens nun nicht mehr dem Aufseher, sondern dem Museumswärter aufgetragen wurde. ${ }^{457}$

Doch welche Möglichkeiten gab es für besagte Fremde und Liebhaber- außer durch direkte persönliche Kontakte - von dem Museum Kenntnis zu erlangen? Aus den ersten 100 Jahren der Existenz der Universität existieren zahlreiche Briefe, Ratgeber und Reflexionen, die, wenn auch nur vereinzelt, Schlaglichter auf das Museum und seine Nutzung werfen. So heißt es beispielsweise bei Ludwig Wallis:

Wer nur den Namen Blumenbach hört, und erfährt, dass das Museum unter seiner Leitung steht, der wird schon zu nicht geringen Erwartungen sich berechtigt glauben, - und er täuscht sich auch nicht! Lobenswerth ist zugleich der hohe Grad von Gemeinnützigkeit dieser vortrefflichen Sammlung von Merkwürdigkeiten aus allen Naturreichen, indem man von einem angesetzten Aufseher sich das Ganze zu beliebigen Stunden gegen eine kleine Erkenntlichkeit (von 2 Gulden; wofür aber 6 Personen das Vergnügen genießen können) zeigen lassen kann: ungleich größeres Interesse gewährt es allerdings, wenn man Blumenbachs eigene Ansichten und Bemerkungen zugleich hören kann. Dieser geistige Genuß wird seinen jedesmaligen Zuhörern in der Naturgeschichte zu Theil. In demselben Gebäude befindet sich auch die Gemälde-Sammlung, das Münz-Cabinet und die ModellKammer. ${ }^{458}$

Ein Besuch des Museums wurde also gestattet, wenn man einen Eintrittspreis, der als „Erkenntlichkeit“ bezeichnet wird, bezahlte. Es werden auch nicht nur Einzelpersonen, sondern auch Gruppen von einem Angestellten durch das Museum geführt. Über

${ }^{456}$ UAG Kur 4 V g 5. Der Text der gesamten Dienstanweisung findet sich in Kap. 2.2.3.

${ }^{457}$ Vgl. die in Kap 5.1 widergegebene Dienstanweisung für den Hussier (UAG Kur 4 V g 5, 22r-23r.)

458 LuDWIG WALLIS, Der Göttinger Student. Mit acht Ansichten. Neudruck der Ausgabe von 1813, Göttingen 1913, 15. 
die Dauer einer solchen Führung erfährt man hingegen nichts. Wallis selbst ist nicht im Besucherbuch des Museums verzeichnet. Seine Schilderungen vermitteln aber den Eindruck, dass er das Museum aus eigener Erfahrung kannte. Eventuell hörte er Naturgeschichte bei Blumenbach und kannte daher die Sammlung. Dafür spricht auch die Bemerkung, dass er eine Führung durch Blumenbach persönlich der eines Angestellten vorzieht.

Andere Berichterstatter hingege erwähnten oft nur die Existenz des Museums. So etwa Thomas Hodgskin, der 1820 über Göttingen schrieb „The only buildings belonging to the university at Göttingen are, the library, a museum, an observatory, and a council-house.“459 Die umfangreichsten Quellen, die überregional über die Bestände des Academischen Museums informierten, sind Blumenbachs Mitteilungen aus den Jahren 1787 und 1788. ${ }^{460}$ Diese sind auch deshalb spannend, weil er darin eine scharfe Abgrenzung des Göttinger Museums zu Raritäten-Kabinetten vornimmt. ${ }^{461}$ Daran anschließend gibt er eine Übersicht über die Highlights unter den Sammlungsstücken und hebt deren Einzigartigkeit hervor.

Erwähnung findet das Academische Museum ferner bei Gustav Klemm, ${ }^{462}$ der ihm unter den Sammlungen bei Bildungsanstalten die erste Stelle einräumt und in kurzen Worten den Bestand des Museums umreißt. Allerdings zeichnet sich dieser Text nicht durch übermäßige Genauigkeit aus. So ist die Entstehung des Museums z.B. auf „um 1774“ datiert.

Friedrich Karl Gottlob Hirsching gibt in seinem bekannten Sammlungsführer umfassende Informationen über öffentliche Sammlungen in Göttingen. ${ }^{463}$ Der Text über

459 Thomas Hodgskin, Travels in the North of Germany: describing the present state of the social and political institutiones, the agriculture, manufactures, commerce, education, arts and manners and the country; particularly in the kingdom of Hannover. Vol. II, Edinburgh 1820, 268.

460 JOHANN FRIEDRICH BlumENBACH, Einige Nachrichten vom academischen Museum zu Göttingen, in: Annalen der Braunschweig-Lüneburgischen Churlande 1 (1787) H. 3, 84-99. JOHANN FRIEDRICH BlumENBACH, Einige Nachrichten vom academischen Museum zu Göttingen, in: Annalen der Braunschweig-Lüneburgischen Churlande 2 (1788) H. 2, 25-35. Vgl. auch GEORG CHRISTOPH LichtenBerg, Etwas vom Academischen Museum in Göttingen, in: Göttinger Taschen Calender (1779), 45-57

461 BlumenBaCH, Mitteilungen, 1787, 87.

462 Vgl. GustaV KLEMM, Zur Geschichte der Sammlungen für Wissenschaft und Kunst in Deutschland, Zerbst 1837, 237.

463 Friedrich KARL GOTTLOB HiRSChING (Hg.), Nachrichten von sehenswürdigen Gemälde- und Kupferstichsammlungen, Münz- Gemmen- Kunst- und Naturalienkabineten, Sammlungen von Modellen, Maschinen, physikalischen und mathematischen Instrumenten, anatomischen Präparaten und botanischen Gärten in Deutschland, nach alphabetischer Ordnung der Oerter. Dritter Band, Erlangen 1789, 156-201. 
das Academische Museum ist allerdings weitgehend identisch mit den zuvor veröffentlichten Arbeiten Blumenbachs, insbesondere dessen Nachrichten vom Academischen Museum zu Göttingen. ${ }^{464}$ Hirsching gesteht dies ein, betont aber, dass er diese Arbeit „mit neuen Zusätzen und Vermehrungen“ versehen habe. ${ }^{465}$ Das Beachtliche an dieser Darstellung ist, dass Hirsching die Universitätssammlungen tatsächlich relativ vollständig erschließt und darüber hinaus auch noch Auskunft über Göttinger Privatsammlungen gibt. ${ }^{466}$

\subsubsection{Wissenschaftliche Zugänglichkeit und Nutzung}

In der bisherigen Abhandlung wurde schon mehrfach darauf hingewiesen, dass die Sammlungen des Museums seit Beginn von Büttners Lehrtätigkeit in den akademischen Unterricht der Naturgeschichte einbezogen worden sind. Während Büttner die Sammlungen in seinen eigenen Räumlichkeiten präsentierte, konnte Blumenbach, der von 1776 an dessen Vorlesungen fortführte, auf die alten Bibliotheksräume zurückgreifen. Ob er seine Kollegien tatsächlich aber dort abhielt oder Objekte - wegen möglicherweise beengten Raumverhältnissen - von dort holen ließ und sie anderenorts präsentierte, ist nicht überliefert.

Nicht erwähnt wurde bislang die Nutzung der Sammlungen im Rahmen der Lehrtätigkeit Heerens. Arnold Hermann Heeren (1760-1842) war Geograph und Historiker. Persönlich eng mit Heyne verbunden, er war dessen Schwiegersohn, wandte er sich am 6. Februar 1803 an die Regierung in Hannover und bat darum,
daß ich während eines jedesmaligen Cursus über die Geographie und Ethnographie meine Zuhörer ein paarmal in die Zimmer des Academischen Musei, welche jenen Apparat ent- halten, führen, und durch Vorzeigung und Erklärung derselben meinen Vortrag deutlicher machen dürfe $\mathrm{u}^{467}$

Diese Bitte wurde Heeren umgehend gewährt. ${ }^{468}$ Es ist möglich, dass Ernst Brandes, der damalige Expedient für Universitätssachen in Hannover tatsächlich „,auf alle Weise das gedachte nützliche Collegium unterstützt zu sehen“469 wünschte, wie er es in seinem Antwortschreiben auf Heerens Gesuch ausdrückt. Aber vielleicht war es auch hilfreich, dass Heeren der Mann von Brandes’ Nichte war. Jedenfalls ist Heeren

\footnotetext{
${ }^{464}$ Vgl. BlumenBACH, Nachrichten 1787.

465 Vgl. HiRSCHING, Nachrichten, 156.

466 Vgl. HiRsCHING, Nachrichten, 201-205.

467 UAG Kur $4 \mathrm{~V}$ g 17, 3r.

${ }^{468}$ Vgl. das Antwortschreiben auf das Gesuch Heerens. UAG Kur 4 V g 17, 1r.

${ }^{469}$ Antwortschreiben auf das Gesuch Heerens. UAG Kur 4 V g 17, 1 r.
} 
die einzige nachweisbare Person, die die Sammlungen des Museums zu Unterrichtszwecken nutzte, ohne am Museums angestellt zu sein. Deshalb wird auch das förmliche Gesuch notwendig gewesen sein. Urban hebt die Relevanz von Heerens Vorlesungen für die Etablierung der Völkerkunde als eigene Fachdisziplin hervor und gibt weiterführende Informationen zu diesem Collegium: ${ }^{470}$ Es war Heeren, der „durch Vorzeigung und Erklärung der damals erst jüngst nach Göttingen gekommenen wertvollen völkerkundlichen Materialien seine Vorlesungen anschaulicher gestaltete. “471 Von 1803 ab findet sich im Vorlesungsverzeichnis der Universität Göttingen jeweils im Sommersemester folgende Ankündigung Heerens:

\begin{abstract}
Allgemeine Länder- und Völkerkunde, oder einen crit. u. systemat. Inbegriff unserer gegenwärtigen Kenntnisse der Erde und der sie bewohnenden Völker, trägt Hr. Prof. Heeren um 6 Uhr M. vor, u. erläutert alles durch einen reichen Vorrath der beßten und neuesten Karten, die er seinen Zuhörern vorlegen wird, und, was die Kleidungen, Waffen, Geräthe, der entfernten Völker betrifft, durch die ethnographische Sammlung in dem königl. Museum. ${ }^{472}$
\end{abstract}

Manfred Urban hat gezeigt, dass diese Lehrveranstaltung über eine sehr lange Zeit hinweg, jeweils im Sommersemester, durchgeführt worden ist. ${ }^{473}$ Er wies nach,

dass u.a. Peter Eckermann, der spätere Vertraute Goethes, und Otto von Bismarck - die beide Heeren als Hochschullehrer sehr schätzten - Heerens Kolleg zur Länder- und Völkerkunde besucht haben. In diesem Zusammenhang werden sie höchstwahrscheinlich auch die ,Erläuterungen des ethnographischen Apparats' durch Heeren miterlebt haben. ${ }^{474}$

Mitteilungen über die wissenschaftliche Arbeit am Objekt sind in den für die vorliegende Arbeit ausgewerteten handschriftlichen Quellen nicht überliefert. Allenfalls in den Göttingischen Anzeigen von gelehrten Sachen finden sich Nachrichten darüber, doch auch hier wird nur selten darauf eingegangen. ${ }^{475}$ Das einzige große Beispiel ist die bereits in Kapitel 1 erwähnte Mumienuntersuchung. Hierbei handelte es sich um die Untersuchung einer Mumie, die der dänische König der Göttinger Akademie der

$470 \mathrm{Zu}$ den Inhalten der anderen Lehrveranstaltungen die die Objekte des Museums nutzten bzw. zu der währenddessen erfolgten Einbettung der Objekte in den Unterricht, sind in den genannten Quellen keine Informationen enthalten. Hier könnten möglicherweise die oben angesprochenen Bestände der Handschriftenabteilung der SUB Göttingen von Nutzen sein, in denen u.a.ein kleines gebundenes Büchlein mit dem Titel „H. Prof. Blumenbachs Vorlesungen über die medic. Litterärgeschichte, Göttingen 1783 (SUB Gö. Cod. Ms. 2004.2) erhalten ist.

471 MANFred URBAN, 200 Jahre Göttinger Cook-Sammlung, Göttingen 1982, 38.

${ }^{472}$ GGA vom 26. März 1803, 501.

473 Vgl. MANFRED URBAN, 200 Jahre Göttinger Cook-Sammlung, Göttingen 1982, 38.

474 MANFred URBAN, 200 Jahre Göttinger Cook-Sammlung, Göttingen 1982, 38.

475 Hierbei handelt es sich um ausgewählte Beitrage. Eine systematische Durchsicht aller 67 Jahrgänge der GGA - die Register sind nicht notwendigerweise zuverlässig - war aus Zeitgründen nicht möglich; exemplarisch wurden die ersten zehn Jahre durchgesehen. 
Wissenschaften überwiesen hatte. Bereits bei der Bekanntgabe dieses Geschenks in den Göttingischen Gelehrten Anzeigen im Februar wurde erklärt, dass die Mumie künftig einen Platz im Academischen Museum finden sollte. An dieser Stelle finden sich ausschließlich beschreibende und schlussfolgernde Bemerkungen. Ganz anders nimmt sich hingegen der im Juni 1781 veröffentlichte Artikel über die Untersuchung der Mumie aus. ${ }^{476}$ Inzwischen hatte Prof. Gmelin zahlreiche chemische Versuche mit ihr angestellt, über die in aller Ausführlichkeit berichtet wird. Allerdings tritt die Mumie hier nicht als Museumsobjekt in Erscheinung. Auch die Göttingischen Anzeigen von gelehrten Sachen liefern also kaum Aufschlüsse über wissenschaftliche Praxis an Museumsobjekten. Dennoch heißt das nicht, dass es sie nicht gegeben hat. So verweist z.B. Plischke auf Erkenntnisse, die Blumenbach seiner Ansicht nach zum Ausbau seiner Dissertation aus den Beständen der Cook-Sammlung gezogen hat:

Den Beziehungen zu Forschungsreisenden, insbesondere denen zu Sir Joseph Banks, verdankte Blumenbach wertvolles neues Beobachtungsmaterial - hingewiesen sei nur auf Südseeschädel von den Reisen des Engländers James Cook, die ihm die Anregung zur Aufstellung einer maliischen Rasse gaben. ${ }^{477}$

Ehlers schreibt ähnliches über Berthold: „Wie die Sammlung Erwerbungen macht, nutzt er [Berthold] diese für Beschreibungen neuer Arten aus, ...“478 Allerdings lassen sich dafür bis 1840 keine Belege finden.

Zu erwähnen bleibt die Möglichkeit, dass das Museum einen gewissen Nutzen als Projektions- und Profilierungsort für die eigenen Arbeiten Blumenbachs hatte. Es ist zwar bis zu einem gewissen Grad hypothetisch, aber durchaus plausibel, dass er die Sammlung zur Visualisierung seiner Theorien benutzt hat. Dies wird durch Pütter gestützt, der schreibt, dass das Museum „nach der dritten Ausgabe des Blumenbachischen Handbuchs geordnet“ gewesen sei. ${ }^{479}$

\footnotetext{
476 Vgl. GGA vom 11. Juni 1781, 569-572.

477 PLISCHKE, Ethnographische Sammlung ,17.

478 EHLERS, Göttinger Zoologen, 37.

479 PÜTTER, Gelehrten-Geschichte (2), 232.
} 


\section{Zusammenfassung und Ausblick}

Betrachtet man die Tendenzen der allgemeinen Sammlungsgeschichte einerseits und die Entstehungsgeschichte des Academischen Museums andererseits, so stellt man fest, dass die Göttinger Sammlung im 18. Jahrhundert ein geradezu klassisches Beispiel für eine Erfolgsgeschichte darstellte. Angesiedelt im Haushalt eines Apothekers und bereits in der Frühaufklärung begonnen, mauserte sich die Sammlung über Jahrzehnte zu einem ansehnlichen Naturalienkabinett, das dann von einer Universität angekauft wurde. Hier erhielt es eigene Räumlichkeiten, wurde (fach-)wissenschaftlichem Personal unterstellt und nach (den damals) neuesten wissenschaftlichen Gesichtspunkten geordnet. Überdies sind aus der Frühzeit des Museums zahlreiche Schenkungen und Ankäufe überliefert, die teils zu einer systematischen Erweiterung der bereits vorhandenen Bestände, teils vor allem zu Reputationsgewinnen führten. Daneben erfährt man, dass die Zugänglichkeit des Museums in verschiedenen Publikationen erwähnt wird und dass die Sammlungen des Museums Besuchern nach vorheriger Anmeldung offen standen, dass sie hindurchgeführt worden sind und nicht sich selbst überlassen blieben. Doch nicht nur „Liebhaber und Fremde“, wie es häufig in zeitgenössischen Quellen heißt, nutzten die Sammlung. Sie wurde zudem in den universitären Unterricht einbezogen. Ferner wurden einige Objekte Gegenstand wissenschaftlicher Untersuchungen.

Bis hierhin erfüllt das Academische Museum alle Kriterien, um die Frage Sammeln für die Wissenschaft? mit einem eindeutigen Ja beantworten zu können. Dies ändert sich jedoch im 19. Jahrhundert. In seiner Konsolidierung durch die französische Herrschaft gestört, entwickelte das Academische Museum im ersten Drittel des 19. Jahrhunderts weder innovative Ordnungssysteme noch eine ausgeprägte Akquisitionstätigkeit. Veränderungen waren vor allem personeller, nicht struktureller Natur. Blumenbach hatte seine Rolle als Initiator, nicht aber seine Position als Oberaufseher abgegeben und wirkte dadurch - in gleichem Maße wie er im 18. Jahrhundert die ,Evolution' des Museums vorangetrieben hatte - entwicklungshemmend. Es trat eine Stagnation ein, die erst seit dem Ende der 1830er Jahre, vor allem durch das Engagement neuen Personals, an das Blumenbach altersbedingt Kompetenzen abzugeben gezwungen war, zurückging. Zu diesem Zeitpunkt wurden die Sammlungen zwar 
noch für Lehrzwecke verwendet, erfüllten aber in keiner Weise mehr wissenschaftliche Standards. Dies sollte sich nach 1840 langsam wieder ändern. Bemerkenswert ist in diesem Zusammenhang auch der Komplex der Sammlungsakquise. Solange das Museum über keinen eigenen Etat verfügte, fehlte mit den Jahren scheinbar die Motivation, sich um außergewöhnliche Sammlungsstücke zu bemühen. Es wurden lediglich kleinere Sammlungseinkäufe vorgenommen. An dieser Stelle sei noch einmal betont, wie wichtig Kontakte und wissenschaftliche Netzwerke für den Bekanntheitsgrad eines Museums waren. Die Korrespondenz, das persönliche Aufeinandertreffen von Gelehrten und Interessierten trugen den Ruf von Ort zu Ort und von Stadt zu Stadt. Wie gezeigt wurde, gab es nur wenige Anzeigen oder Mitteilungen in den Zeitschriften der Zeit, die über das Academische Museum informierten.

Noch eine andere Entwicklungslinie zeichnet sich ab: Der Weg des Academischen Museums vom Naturalienkabinett zu spezialisierten Einzelsammlungen verläuft annähernd strukturhomolog zur Ausdifferenzierung der Einzeldisziplinen. Dabei ist das Museum nicht nur ein Abbild der Entstehung von Einzeldisziplinen. Seine Aufseher trugen vielmehr maßgeblich zu den Ausdifferenzierungsprozessen bei. So gilt bereits Blumenbach als Stammvater gleich zweier medizinischer Fachrichtungen.

Im Rahmen einer objektorientierten Darstellung könnte versucht werden nachzuvollziehen, welchen Wandel die Objekte z.B. durch die jeweiligen Ortswechsel erfuhren oder auch dadurch, dass sich das Wissen über die Objekte veränderte, bspw. wenn die Beziehungen zum Unsichtbaren verloren gingen oder neue entstanden oder auch die ihnen zugeschriebenen Bedeutungen Transformationen erfuhren.

Die Auswertung des Besucherbuches macht deutlich, wie vielfältige Ergebnisse sich mit dieser Quelle erzielen lassen. Sie müssen jedoch einen breiteren Kontext eingebettet werden. Dazu wäre unter anderem ein Abgleich der bisher gewonnenen Erkenntnisse mit anderen Universitätssammlungen, z.B. Erlangen, Würzburg oder Marburg notwendig, um so eine angemessene Kontextualisierung für das Göttinger Museum schaffen zu können. Denn sicherlich hat in gewissem Grad Rupke recht, wenn er konstatiert:

„Yet the Museum [...] never amounted to much, certainly not by comparison with Georges Cuvier's (1769-1832) in Paris, Richard Owen’s (1804-1892) in London or even 
Henry Wentworth Acland's (1815-1900) in Oxford, where the University Museum represented an architectural palace which significantly helped nurture the sciences [...]”. ${ }^{480}$

Es stellt sich jedoch die Frage, ob der Vergleichsmaßstab nicht zu hoch angesetzt ist und ob ein Vergleich mit anderen deutschen Universitätssammlungen nicht zu einem anderen Ergebnis führen würde. Hier könnte man unter Zuhilfenahme mikrogeschichtlicher Methoden die als allgemein gültig angesehenen Tendenzen der Geschichte des Sammelns in Bezug auf das universitäre Sammeln in Frage stellen. Eventuell ließen sich das Konzept der hier erstellten Datenbank auf Besucherbücher weiterer Universitätssammlungen übertragen.

Ein weiteres reizvolles Thema für eine Studie wäre es ein Gesamtbild der Göttinger Museumslandschaft zu schaffen. ${ }^{481}$ Um diese angemessen fassen zu können, fehlen vor allem noch Untersuchungen zum Academischen Museum in der Zeit nach 1840 sowie zum 1877/78 bezogenen Naturhistorischen Museum.

480 NiCOLAAs RupKe, The Göttingen Location, in: Ders. (Hg.), Development of the Natural Sciences, 19-32, 28.

481 Eine erste Grundlage dafür liefert die kürzlich fertig gestellte Arbeit von Heinke Lang zum Städtischen Museum Göttingen. Vgl. HEINKE LANG, Ein Institut des Bildungsbürgertums? Die Anfänge des Göttinger Stadtmuseums (unveröffentlichte Staatsexamensarbeit), Göttingen 2004. 


\section{Anhang A: Der Catalogus Musei Academici}

[Bei der folgenden Abschrift handelt es sich um eine Wiedergabe der Ordnungskategorien, die Blumenbach der Erstausfertigung des Catalogus Musei Academici zugrunde legte. Ein Abgleich mit der von anderer Hand und in Schönschrift ausgeführten Zweitausfertigung fand nicht statt. Während die Orthographie derjenigen des Originals entspricht, finden Seiten- oder Zeilenumbrüche hier keine Berücksichtigung.

Der Katalog ist in hellbrauner Pappe eingebunden, und weist nur auf dem Rücken eine Beschriftung auf (goldene Schrift auf rotem Grund). Bei der Aufzählung der Objekte befindet sich die Nummerierung weit am linken Rand. Es kann wohl davon ausgegangen werden, dass die Auflistung auf einzelnen Blättern erfolgte, die zu einem späteren Zeitpunkt gebunden wurden.

Der Catalogus Musei Academici ist ein reiner Text-Katalog, der keinerlei Abbildungen, schematische Zeichnungen o.ä. enthält. In der Regel handelt es sich um kurze, meist sogar nur einzeilige Beschreibungen der Objekte, in denen auf ihre äußere Form und - soweit bekannt - auf ihren Herkunftsort eingegangen wird. Es finden sich keine quantitativen Angaben wie Gewicht oder Länge; dies lässt Beobachtung und Beschreibung als die der Katalogproduktion zugrunde liegende Praktiken erkennen. An einer Stelle weicht Blumenbach von seinem Schema ab und schreibt einen summarischen Text zum Herbarium Vivum. Hier und an ein, zwei weiteren Stellen gibt der Katalog Aufschluss darüber wann, wie und durch wen die Objekte in die Sammlung gelangt sind. Die in Klammern hinter den Unterkategorien aufgeführten Nummern verweisen auf die Nummerierung der einzelnen Objekte. Sie sollen eine Orientierung zum Umfang der einzelnen Sparten bieten.] 
Kieselartige Steine

Edelsteine

Diamanten [Nummern 1-40]

Rubinen [41-80]

Topasen [81-302]

Saphiren [303-333]

Smaragde [334-353]

Hyacinthen [354-401]

Berylle [402-407]

Amethysten [408-467]

Chrÿsolithen [468-506]

Turmaline [507-510]

Granaten [511-684]

Jargons [685-696]

Opale [697-732]

Katzenaugen [733-749]

Quarz-Crystalle

feine Chrystalle oder unächte Diamanten [750-913]

Rauch Topase [914-974]

Quarz Crystall Drusen

A. Die Crystallen mit ihrem Prisma oder corpus intermedium [975-1167]

B. Die blosen Apues [?] der Crystallen ohne Prisma oder

Corpus intermedium [1168-1344]

Roher Quarz [1345-1370]

Quarzkiesel [1371-1379]

Körniger Quarz [1380-1388]

Blättriger Quarz [1389-1400]

Achate [1401-1753]

Praser und Chrÿsopraser [1754-1775]

Carneol [1776-1833]

Chalcedon und Cacholong [1834-1917]

Onyx [1918-1946]

Feuerstein [1947-1965]

Hornstein [1966-1984]

gefärbte Kiesel [1985-2056]

Jaspis [2057-2255]

Sandstein [2256-2273]

Filtrirstein [2274-2275]

Granit [2276-2308]

Porphyr [2309-2312]

Wacke [2313-2314]

Poudding-Stone [2315-2317]

sogenannte Donnerkeile [2318-2322]

Thonigte Steine

Thon [1-27]

Porcellanerden und Porcellanversuche [28-75] 
Mergel [76-170]

Umbra [171-176]

Bolus [177-200]

Terra Sigillata [201-240]

Steinmark [241-258]

Speckstein [259-269]

Nierenstein [270-272]

Lavezzi oder Tropfstein [273-278]

Serpentinstein [279-340]

Glimmer [341-419]

Calk [420-429]

Waßerbley oder Molybdæna [430-449]

Slut oder Rußisches Jungfern Glas [450-458]

Amianth und Asbest [459-497]

Schiefer [498-520]

schwarze Kreite [521-528]

Probirstein [529-532]

Gneis [533-578]

Letten [579-586]

Kalkartige Steine

Kalkstein [1-39]

Marmor [40-154]

Lapis lazuli [155-192]

Kreite [193-197]

Mondmilch und Bergmehl [198-199]

Tophstein, Incrustate etc. [200-287]

Tropfstein oder Stalachit [288-339]

Eisenblüthe [240-349]

KalkSpat

A. cubischer KalkSpat [350-353]

B. Doppelspat oder sogenannter Isländischer Crystal [354-367]

C. Dreÿseitige KalkSpat Spitzen ohne Prisma [368-378]

D. sogenannte Schweiszæhne [379-474]

E. sogenannte Kanonen Drusen [475-575]

F. KalkSpat mit 6seitiger Säule, 6seitiger Spitze, und 6seitiger Endfläche [576]

G. KalkSpat mit 6seitiger Säule, 3seitiger Spitze, und 3seitiger Endfläche [577-580]

H. KalkSpat mit 6seitiger Säule, woran sich am Ende 3 Seiten mit schrägen Facetten umschlagen. mit 6seitiger Endfläche [581-583]

I. KalkSpat mit 6seitiger Säule und 3seitiger Spitze [584-600]

K. Sogenannter NagelkopfSpat [601-618]

L. Blätter KalkSpat in ganz dunnen klaren 6eckigen Scheibgen [619-620]

M. KalkSpat mit 6seitigen Säulen wie die Kanonen Drusen. Mit- 
ten aus der Endfläche steigt aber eine kurze 6seitige Spitze heraus deren Schärfen auf die Flächen der Säule und vice versa, paßen. [621]

N. KalkSpat mit langer 9seitiger Säule und 6seitiger EndSpitze [622]

O. Flachgedruckte KalkSpatCrystallen fast wie Degenklingen [623-625]

P. Irregulairer Kalkspat mit in die Länge verschobenen Krystalen [626-633]

Q. andre irregulaire Kalkspatdrusen [634-659]

R. sogenannter Ludus Helmontii [660]

S. KalkSpat Bruchstücken [661-672]

Gyps [673-706]

Alabaster [707-761]

GÿpsSinter [762-764]

Selenit [765-774]

Frauenfir [775-788]

GÿpsSpat [789-818]

würfliger Fluß Spat [819-916]

schwehrer Spat [917-1038]

HaarDrusen von schweren Spat [1039-1065]

Bononischer Stein [1066-1071]

Zeolith [1072-1080]

Versteinerungen

versteinte Knochen [1-29]

versteinte Zähne von Säugethieren [30-38]

Glossopetrae [39-69]

versteinte Fische [70-113]

Busoniten [114-115]

Türkiße [116-154]

versteinte Krebse [155-160]

Trilobiten oder sogenannte Cacadumuscheln (Dudleyfossils) [161-166]

versteinte Seeigel [167-283]

JudenNadeln u.a. SeeIgelStacheln [284-322]

versteinte Seesterne [323]

Vermiculiten [324-326]

versteinte viel schaalichte Muscheln, See-Tulpen [327-328]

versteinte zweyschaalichte Muscheln, Venusmuscheln [329]

Chamae [330-400]

KammMuscheln [401-454]

Archen [455-459]

Ostrauten [460-493]

Bucarditen [494-497]

Grÿphiten [498-506]

PantoffelMuscheln [507-512]

Anomien [513-520]

Cerebratula [521-590]

Hÿsterolithen [591-606] 


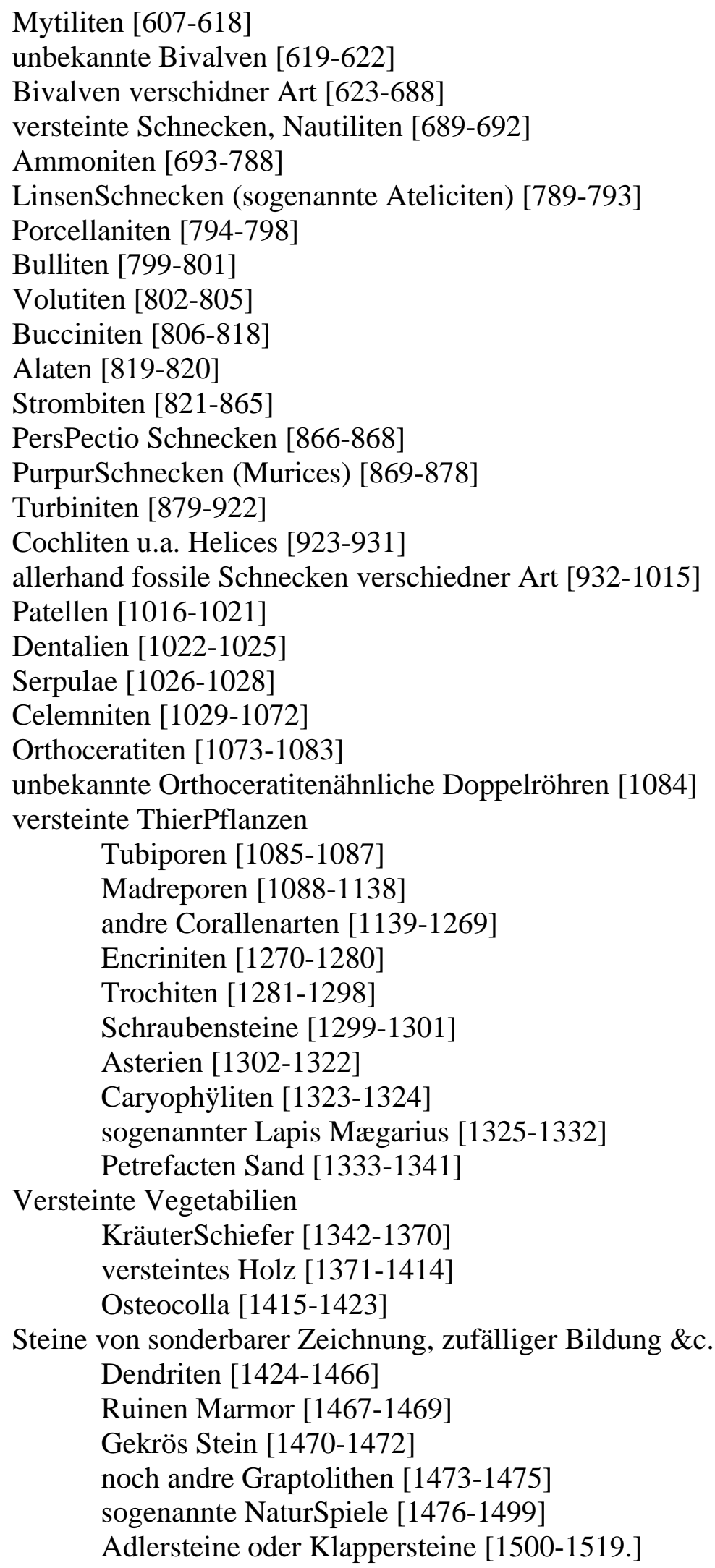


Metalle

ganze Metalle

Gold

gediegen Gold [1-108]

Waschgold [109-117]

güldische Kiese [ohne Nummern]

güldischer Quarz [118-134]

güldische Schiefer [135-144]

güldische Kiese [145-192]

Goldhaltige Minern verschiedner Art [193-315]

Sinopel [316]

Platina del Pinto [317]

gediegen Silber [318-590; an erster Stelle steht die 1782 gestohlene Andreasberger Silberstufe (mit Gewichtsund Wertangaben)]

HornErze [591-596]

Glas Erzt [597-684]

Rothgülden [685-776]

Weißgülden [777-885]

Gänseköthig Erzt [886]

Feder Erzt [887-891]

Brand Erzt [892-898]

allerhand Silber Erzte verschiedner Art [898-1004]

Kupfer

gediegen Kupfer [1005-1151]

Cæment Kupfer [1152-1174]

KupferGlasErzte [1175-1182]

Kupfer Lasur [1184-1253, sic]

KupferFahlErzt [1254-1397]

Kupfer LeberErzte [1398-1421]

Kupfer Kies [1492-1561]

Kupfer Schiefer [1562-1601]

KupferErzte verschiedner Art [1602-1872]

Präparate von Kupfer [1873-1884]

Zinn

gediegen Zinn [1885-1891]

Zinn Graupen und Zinn Granaten [1892-2111]

Zinn-Schörl [2112-2124]

Zwitter [2125-2202]

Wolfram [2203-2208]

ZinnErzte verschiedner Art [2209-2452]

Blë̈

gediegen Bleÿ [2453-2457]

Bleÿglanz [2458-2778]

BleySpat [2779-2826]

Bleÿerzte verschiedner Art [2827-2850]

Bleÿglas [2851-2854] 


\section{Eisen}

gediegen Eisen [2855-2864]

BohnenErzt [2865-2868]

Eisenmann [2869-2878]

Eisenram [2879-2888]

Magnet [2889-2895]

Eisenstein [2896-3198]

Glaskopf und Blutstein [3199-3282]

Braunstein [3283-3318]

Eisenspat [3319-3387]

Eisenerzte verschiedner Art [3388-3416]

Qvecksilber

gediegenes Qvecksilber [3417-3419]

Zinnober u.a. Qvecksilber Minern [3420-3598]

Halbmetalle

Kupfer Nickel [1-18]

Spies Glas [19-102]

Wismuth [103-146]

Zink [147-238]

Kobalt [239-869]

Arsenik [870-906]

Salze

Vitriol [1-34]

Alaun [35-57]

Steinsalz [58-65]

Salmiac [66-67]

Erdharze

Ambra [1-4]

Bernstein [5-39]

Turf [40-43]

Steinkohlen [44-83]

Schwefel

gediegner Schwefel [84-107]

Schwefelkies [108-324]

Vulcanische Producte

Laven [1-12]

Verglasungen [13-17]

Basalt [18-22]

Puzzolana [23-24]

Tuffa [25-27]

Trapp [28-31]

Bimsstein [32-36] 
Herbarium Vivum

Die große Kräuter Sammlung des Musei enthält auser den zahlreichen Pflanzen die Herr Professor Büttner auf seinen Reisen durchs Nordliche Europa und nachher beÿ seinem Aufenthalt in Goettingen gesammlet; und auser einer ansehnliches Collection ausländischer Gewächse die nach der Hand aus Holland dazu erkauft worden, - noch 82 Volumina, welche aus des Hannöverischen Leib Medicus von Hugo Verlaßenschaft auf die UniversitætsBibliothek und von da ins Museum gekommen sind; worunter sich 12 Volumina Malbarischer Pflanzen, wozu die Etiquetten in Malabraischer Sprache auf Palmblätter geschrieben sind - sehr viele andre exotische Gewächse, die der große Pariser Botaniste Vaillant für Herrn von Hugo gesammlet - viele Bände Schweizer Pflanzen von der Hand des Herrn Von Haller - eine große Collection aus dem Leidner Garten - eine andre von Englischen Gewächsen u. s. w. befinden[.]

Andre Vegetabilische Stücke [1-108; Hölzer, Früchte, Seetang...]

Säuge Thiere

INERMIS. Der Mensch [1-107]

PITHECI. Affen - Paviane - MeerKatzen und Makis [108-117]

BRADYPODA. Faulthiere und Ameisenbären [118-122]

SCLERODERMATA. Igel und Stachelschweine, formosische Teufelgen und Panzerthiere [123-129]

CHIROPTERA. Fledermäuse [130-132]

GLIRES - Eichhörngen - Marmotten - Mäuse - Hasen - Wiesel und verwandte Thiere - [133-173]

FERAE. Reisende Thiere [174-193]

SOLIDUNGULA - Säugethiere mit Hufen [194-197]

BISULCA. Säugethiere mit gespaltnen Klauen [198-256]

BELLUAE. Elephant, Rhinocer, Nilpferd \&c [257-276]

PALMATA. Säugethiere mit Ruder-Füßen [277-286]

CETACEA Wallfische [287-307]

Vögel

ACCIPITRES. Raubvögel [1-9]

LEVIROSTRES Papageÿen, Pfefferfraße p [10-21]

PICI. Spechte \&c [23-32]

ANSEREN Schwimmvögel [33-36]

STRUTHIONES. Straus, Casuar \&c [37-42]

GRALLTA [?] SumpfVögel [43-48]

GALLINAE Hünerarten [49-55]

CORACES. Raben. Heher p [56-58]

PASSERES Sangvögel [59-73] 
Amphibien

REPTILES. Vierfüßige Amphibien [1-74]

SERPENTES. Schlangen [75-125]

NANTES. Amphibien mit Floßfedern [126-197]

Fische

APODES ohne Buchfloßfedern [1-3]

IUGULARES mit Bauchfloßfedern vor den Brustfloßen [4-11]

THORACICI mit Bauchfloßfedern grade unter den Brustfloßen [12-26]

ABDOMINALES mit Bauchfloßfedern hinter den Brustfloßen [27-58]

Insecten

COLEOPTERA. Käfer \&c [1-36]

HEMIPTERA. Heuschrecken, Wanzen \&c [37-58]

LEPIDOPTERA Schmetterlinge [59-71]

NEURUPTERA WaßerJungfern \&c [72-77]

HYMENOPTERA, Wespen, Bienen \&c [78-89]

DIPTERA Insecten mit zwey Flügeln [90-100]

APTERA ungeflügelte Insecten [101-151]

Würmer

MOLLUSCA Weiche oder Knorplichte Würmer [1-73]

Testacea - Schaalthiere - Conchylien

Einschaalige Conchylien - Cochleae [1-1238]

Zweyschaalige Conchylien (Conchae) [1239-1453]

Vielschaalige Conchylien [1454-1476]

ThierPflanzen, Zoophyten [1-55]

Materia medica

Eine vollständige Sammlung aller in

der Medicin brauchbaren oder doch

ehedem für brauchbar heilsam ge-

haltenen einfachen Arzneÿ-Mittel

aus allen dreÿ Reichen, besonders

alle officinellen Wurzeln, Rinden,

Hölzer, Blätter, Früchte, Säffte,

Harze u. s. w.

Corpus pharmaceuticum

Eine Sammlung zusammenge-

setzter Arzneÿ-Mittel und phar-

maceutische Präparate, als

Oele, Salze u. s. w.

Artefacten [1-66] 


\section{Anhang B: Auswertung des Besucherbuchs}

Die Datenbasis für die nachstehend vorgenommenen Auswertungen bilden die Besuchereinträge im Fremdenbuch des Academischen Museums Göttingen im Zeitraum vom 5. Juli 1808 bis zum 18. März 1821. Hier lassen sich mindestens 3099 Besucher in 2926 Einträgen nachweisen. Die Einschränkung mindestens ist darauf zurückzuführen, dass beim Eintrag „mit Familie“ jeweils zwei Besucher angenommen werden. Dies betrifft 21 Einträge. Bei den Einträgen „mit Gesellschaft“ (vier Einträge) und „mit Kindern“ bzw. „Zöglingen“ (zwei Einträge) wurden jeweils drei Besucher angenommen.

Anhand der für diese Arbeit entwickelten Datenbank lassen sich weit mehr Auswertungen vornehmen, als an dieser Stelle vorgestellt werden können. Einige Ergebnisse exemplarischer Auswertungen, auf die in Kap. 5.2.2 direkt Bezug genommen wurde, sind in diesem Anhang zusammengestellt. 


\section{Überblick Gesamtzeitraum}

\section{Nach Jahren}

\begin{tabular}{|l|r|r|r|r|}
\hline \multicolumn{1}{|c|}{ Zeit } & Gesamtzahl & Männlich & Weiblich & Unklar \\
\hline 1808 & 141 & 72 & 26 & 43 \\
\hline 1809 & 165 & 85 & 10 & 70 \\
\hline 1810 & 190 & 100 & 10 & 80 \\
\hline 1811 & 257 & 143 & 27 & 87 \\
\hline 1812 & 164 & 82 & 9 & 73 \\
\hline 1813 & 175 & 86 & 13 & 76 \\
\hline 1814 & 325 & 255 & 21 & 49 \\
\hline 1815 & 289 & 212 & 14 & 63 \\
\hline 1816 & 207 & 140 & 20 & 47 \\
\hline 1817 & 211 & 134 & 13 & 64 \\
\hline 1818 & 313 & 206 & 12 & 95 \\
\hline 1819 & 296 & 222 & 21 & 53 \\
\hline 1820 & 353 & 275 & 20 & 58 \\
\hline 1821 & 13 & 13 & 0 & 0 \\
\hline Gesamt & $\mathbf{3 0 9 9}$ & $\mathbf{2 0 2 5}$ & & $\mathbf{8 5 8}$ \\
\hline \hline
\end{tabular}

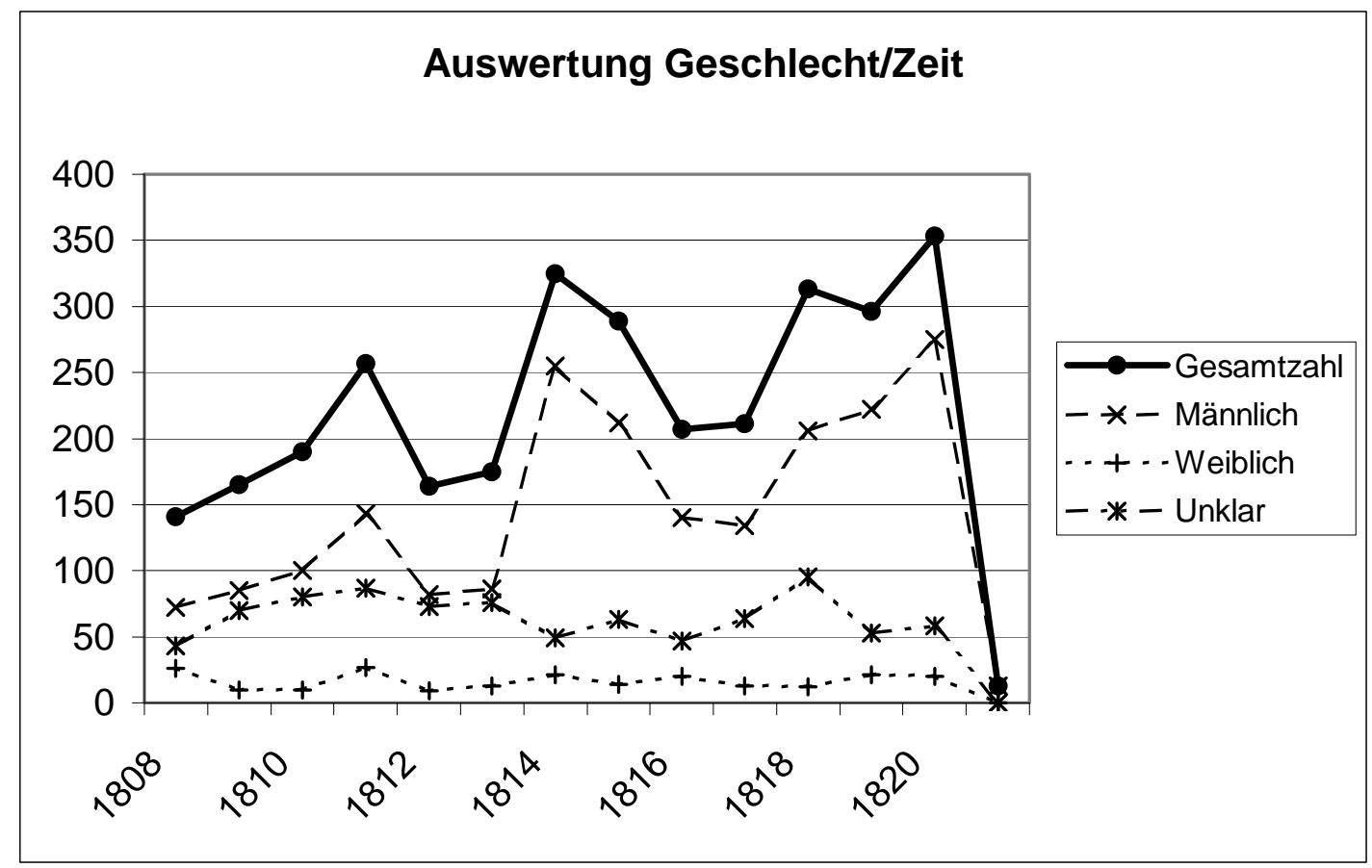


Nach Monaten

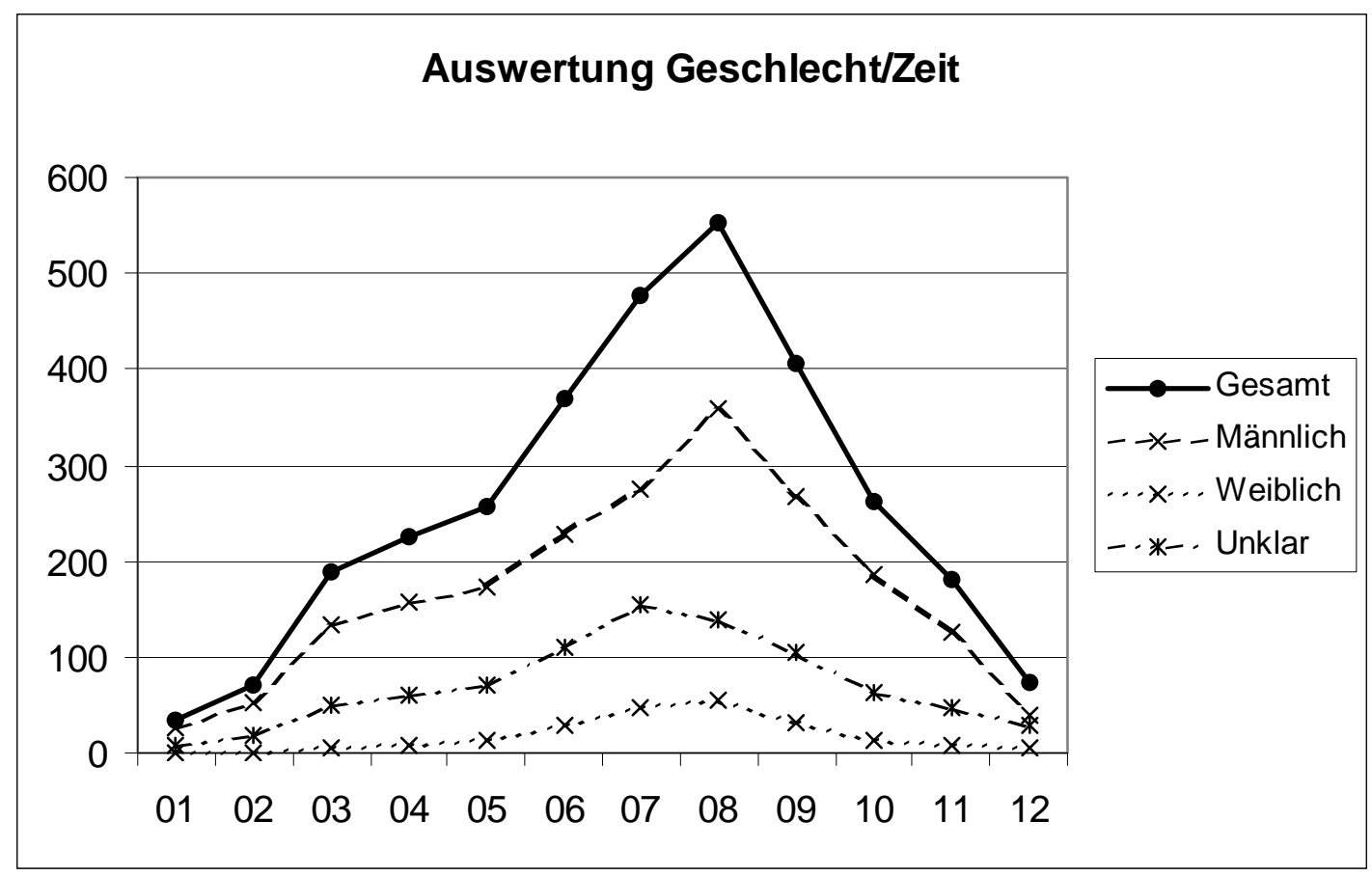

\begin{tabular}{|l|r|r|r|r|}
\hline \multicolumn{1}{|c|}{ Monat } & \multicolumn{1}{c|}{ Gesamt } & \multicolumn{1}{c|}{ Männlich } & Weiblich & Unklar \\
\hline 01 & 35 & 27 & 0 & 8 \\
\hline 02 & 71 & 52 & 1 & 18 \\
\hline 03 & 189 & 133 & 5 & 51 \\
\hline 04 & 225 & 157 & 9 & 59 \\
\hline 05 & 257 & 174 & 12 & 71 \\
\hline 06 & 369 & 229 & 29 & 111 \\
\hline 07 & 477 & 275 & 48 & 154 \\
\hline 08 & 553 & 359 & 54 & 140 \\
\hline 09 & 405 & 267 & 32 & 106 \\
\hline 10 & 263 & 187 & 14 & 62 \\
\hline 11 & 182 & 126 & 8 & 48 \\
\hline 12 & 73 & 39 & 4 & 30 \\
\hline Gesamt & 3099 & 2025 & 216 & 858 \\
\hline \hline
\end{tabular}




\section{Nach Wochentagen}

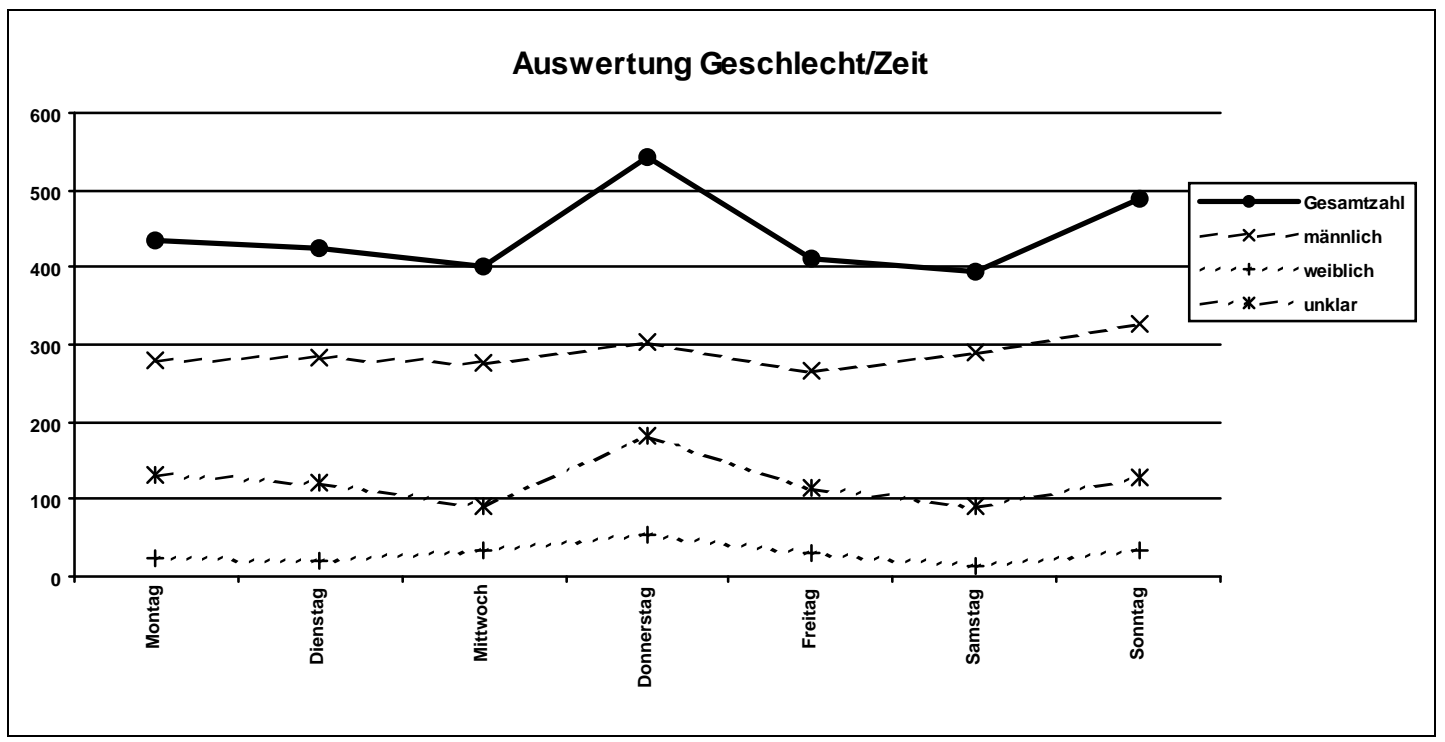

\begin{tabular}{|l|r|r|r|r|r|r|r|r|}
\hline Wochentag & \multicolumn{2}{|c|}{ Gesamtzahl } & \multicolumn{3}{|c|}{ Männlich } & \multicolumn{2}{|c|}{ Weiblich } & \multicolumn{2}{|c|}{ Unklar } \\
\hline Montag & 436 & $14,1 \%$ & 280 & $9,0 \%$ & 24 & $0,8 \%$ & 132 & $4,3 \%$ \\
\hline Dienstag & 424 & $13,7 \%$ & 282 & $9,1 \%$ & 21 & $0,7 \%$ & 121 & $3,9 \%$ \\
\hline Mittwoch & 401 & $12,9 \%$ & 276 & $8,9 \%$ & 35 & $1,1 \%$ & 90 & $2,9 \%$ \\
\hline Donnerstag & 542 & $17,5 \%$ & 305 & $9,8 \%$ & 54 & $1,7 \%$ & 183 & $5,9 \%$ \\
\hline Freitag & 412 & $13,3 \%$ & 265 & $8,6 \%$ & 32 & $1,0 \%$ & 115 & $3,7 \%$ \\
\hline Samstag & 394 & $12,7 \%$ & 289 & $9,3 \%$ & 15 & $0,5 \%$ & 90 & $2,9 \%$ \\
\hline Sonntag & 490 & $15,8 \%$ & 328 & $10,6 \%$ & 35 & $1,1 \%$ & 127 & $4,1 \%$ \\
\hline Summe & $\mathbf{3 0 9 9}$ & $\mathbf{1 0 0 , 0} \%$ & $\mathbf{2 0 2 5}$ & $\mathbf{6 5 , 3 \%}$ & $\mathbf{2 1 6}$ & $\mathbf{7 , 0} \%$ & $\mathbf{8 5 8}$ & $\mathbf{2 7 , 7 \%}$ \\
\hline \hline
\end{tabular}




\section{Nach Entfernung}

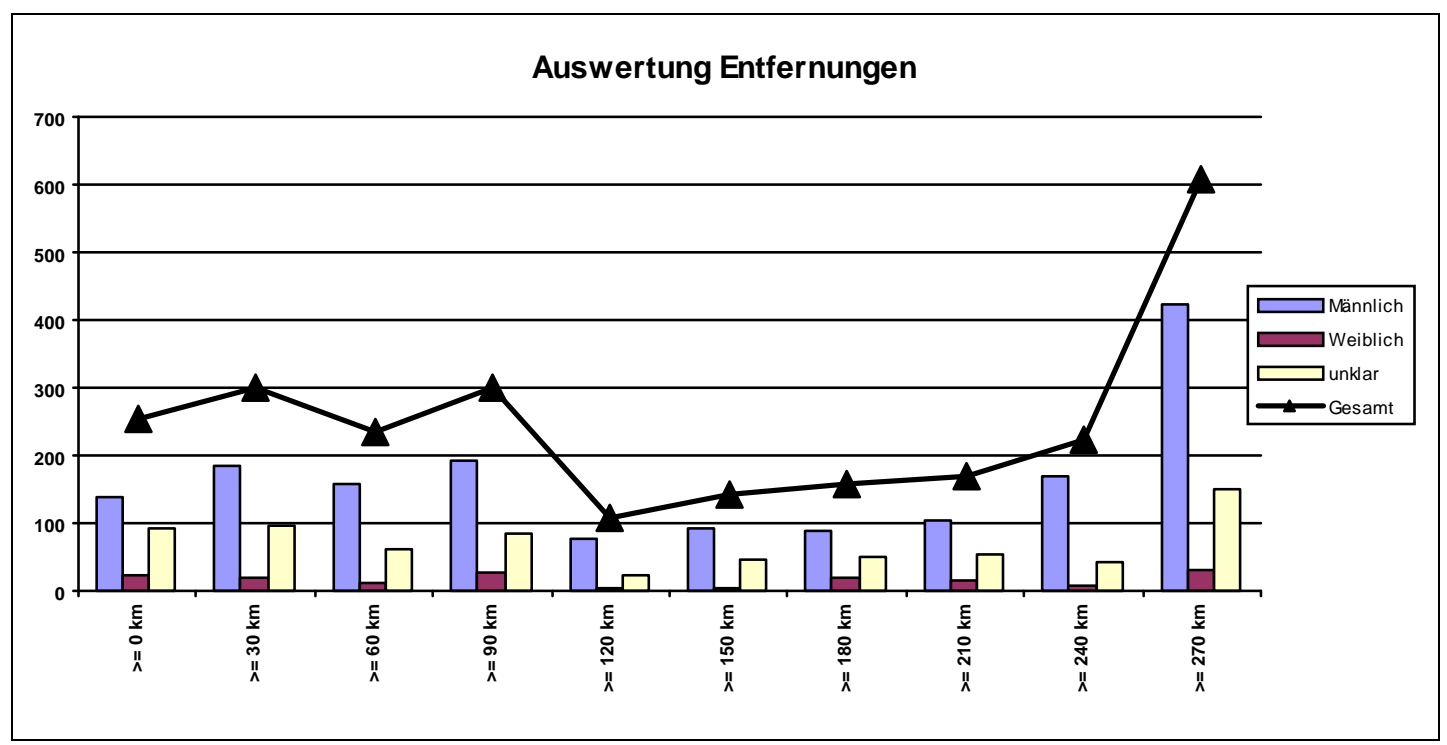

\begin{tabular}{|l|r|r|r|r|}
\hline \multicolumn{1}{|c|}{ von } & \multicolumn{1}{c|}{ Gesamt } & Männlich & Weiblich & unklar \\
\hline 0 & 255 & 140 & 24 & 91 \\
\hline 30 & 299 & 184 & 20 & 95 \\
\hline 60 & 233 & 158 & 12 & 63 \\
\hline 90 & 300 & 191 & 26 & 83 \\
\hline 120 & 107 & 78 & 5 & 24 \\
\hline 150 & 142 & 94 & 2 & 46 \\
\hline 180 & 156 & 88 & 19 & 49 \\
\hline 210 & 171 & 104 & 15 & 52 \\
\hline 240 & 222 & 171 & 9 & 42 \\
\hline 270 & 606 & 425 & 31 & 150 \\
\hline Summe & $\mathbf{2 4 9 1}$ & $\mathbf{1 6 3 3}$ & $\mathbf{1 6 3}$ & $\mathbf{6 9 5}$ \\
\hline \hline
\end{tabular}




\section{Adelige Besucher}

\begin{tabular}{|l|r|r|r|r|r|r|r|r|}
\hline & \multicolumn{2}{|c|}{ Gesamtzahl } & \multicolumn{2}{|c|}{ Männlich } & \multicolumn{2}{|c|}{ Weiblich } & \multicolumn{2}{|c|}{ Unklar } \\
\hline Adelige & 376 & $12,1 \%$ & 257 & $8,2 \%$ & 39 & $1,3 \%$ & 80 & $2,6 \%$ \\
\hline [Kein Eintrag] & 2723 & $87,9 \%$ & 1768 & $57,1 \%$ & 177 & $5,7 \%$ & 778 & $25,1 \%$ \\
\hline Summe & 3099 & $100,0 \%$ & 2025 & $65,3 \%$ & 216 & $7,0 \%$ & 858 & $27,7 \%$ \\
\hline \hline
\end{tabular}

\begin{tabular}{|l|r|r|r|r|r|r|r|r|}
\hline \multicolumn{1}{|c|}{ Titel } & Gesamtzahl & \multicolumn{2}{|c|}{ Männlich } & \multicolumn{2}{|c|}{ Weiblich } & \multicolumn{2}{|c|}{ Unklar } \\
\hline Freiherr & 26 & $6,9 \%$ & 24 & $6,4 \%$ & 2 & $0,5 \%$ & 0 & $0,0 \%$ \\
\hline Fürst & 2 & $0,5 \%$ & 1 & $0,3 \%$ & 1 & $0,3 \%$ & 0 & $0,0 \%$ \\
\hline Graf & 33 & $8,8 \%$ & 27 & $7,2 \%$ & 6 & $1,6 \%$ & 0 & $0,0 \%$ \\
\hline Herzog & 1 & $0,3 \%$ & 1 & $0,3 \%$ & 0 & $0,0 \%$ & 0 & $0,0 \%$ \\
\hline Hofrath & 9 & $2,4 \%$ & 7 & $1,9 \%$ & 0 & $0,0 \%$ & 2 & $0,5 \%$ \\
\hline Hofrath und Ritter & 1 & $0,3 \%$ & 1 & $0,3 \%$ & 0 & $0,0 \%$ & 0 & $0,0 \%$ \\
\hline Hofrath von & 1 & $0,3 \%$ & 1 & $0,3 \%$ & 0 & $0,0 \%$ & 0 & $0,0 \%$ \\
\hline Markgraf & 1 & $0,3 \%$ & 1 & $0,3 \%$ & 0 & $0,0 \%$ & 0 & $0,0 \%$ \\
\hline Prinz & 1 & $0,3 \%$ & 1 & $0,3 \%$ & 0 & $0,0 \%$ & 0 & $0,0 \%$ \\
\hline Ritter & 3 & $0,8 \%$ & 2 & $0,5 \%$ & 0 & $0,0 \%$ & 1 & $0,3 \%$ \\
\hline Vicomte & 3 & $0,8 \%$ & 3 & $0,8 \%$ & 0 & $0,0 \%$ & 0 & $0,0 \%$ \\
\hline von & 295 & $78,5 \%$ & 188 & $50,0 \%$ & 30 & $8,0 \%$ & 77 & $20,5 \%$ \\
\hline Summe & $\mathbf{3 7 6}$ & $\mathbf{1 0 0 , 0 \%}$ & $\mathbf{2 5 7}$ & $\mathbf{6 8 , 4 \%}$ & $\mathbf{3 9}$ & $\mathbf{1 0 , 4 \%}$ & $\mathbf{8 0}$ & $\mathbf{2 1 , 3 \%}$ \\
\hline \hline
\end{tabular}

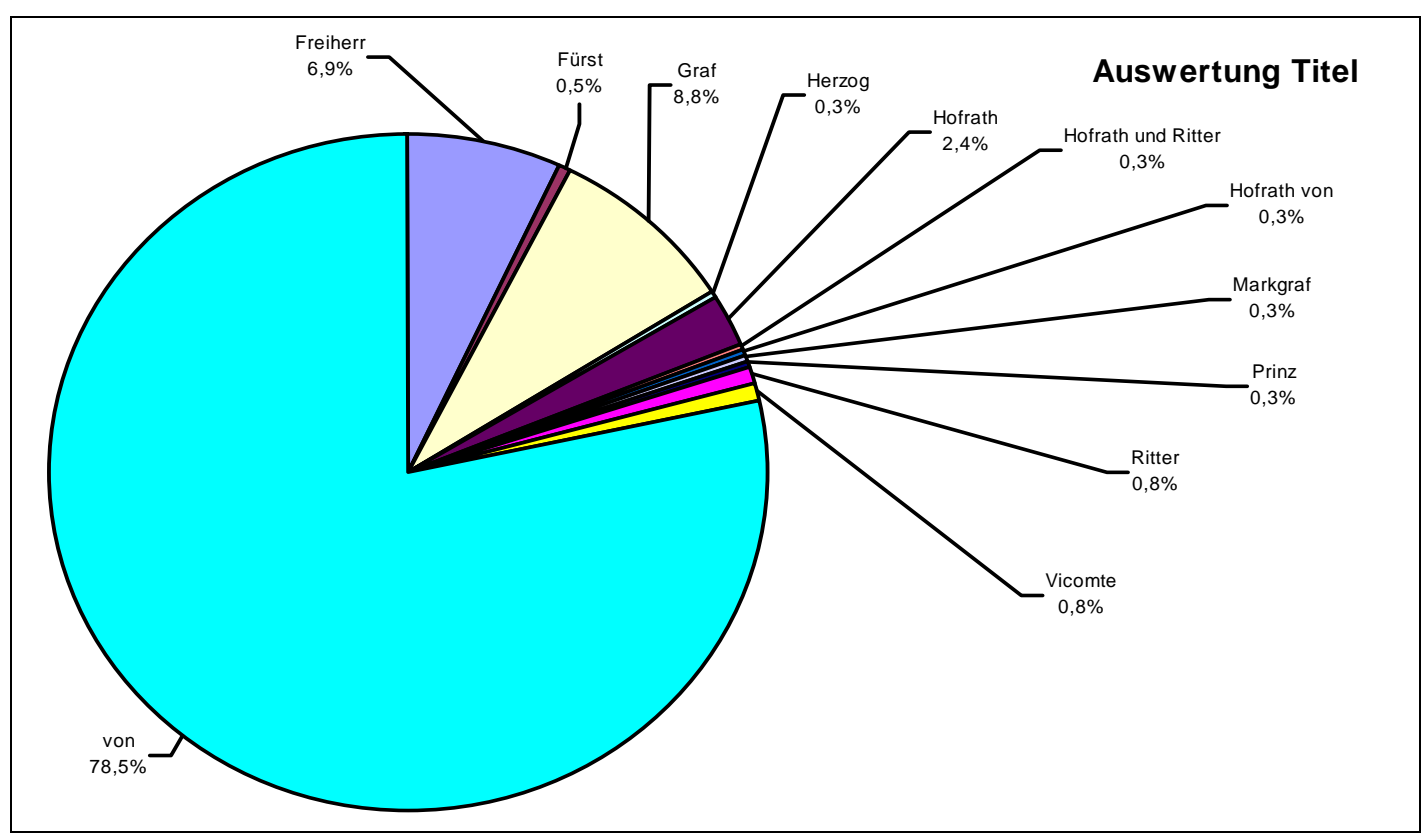




\section{Besucher mit akademischer Ausbildung}

\begin{tabular}{|l|r|r|r|r|r|r|r|r|}
\hline & \multicolumn{2}{|c|}{ Gesamtzahl } & \multicolumn{2}{|c|}{ Männlich } & \multicolumn{2}{|c|}{ Weiblich } & \multicolumn{2}{|c|}{ Unklar } \\
\hline Akademiker & 703 & $22,7 \%$ & 687 & $22,1 \%$ & 10 & $1,4 \%$ & 6 & $0,2 \%$ \\
\hline [Kein Eintrag] & 2396 & $77,3 \%$ & 1338 & $43,2 \%$ & 206 & $6,6 \%$ & 852 & $27,5 \%$ \\
\hline Summe & $\mathbf{3 0 9 9}$ & $\mathbf{1 0 0 , 0} \%$ & $\mathbf{2 0 2 5}$ & $\mathbf{6 5 , 3 \%}$ & $\mathbf{2 1 6}$ & $\mathbf{7 , 0 \%}$ & $\mathbf{8 5 8}$ & $\mathbf{2 7 , 7 \%}$ \\
\hline \hline
\end{tabular}

\begin{tabular}{|l|r|r|r|r|r|r|r|r|}
\hline $\begin{array}{c}\text { Akademischer } \\
\text { Grad }\end{array}$ & \multicolumn{2}{|c|}{ Gesamtzahl } & \multicolumn{2}{c|}{ Männlich } & \multicolumn{2}{c|}{ Weiblich } & \multicolumn{2}{c|}{ Unklar } \\
\hline Assessor & 1 & $0,1 \%$ & 1 & $0,1 \%$ & 0 & $0,0 \%$ & 0 & $0,0 \%$ \\
\hline Candidat & 49 & $7,0 \%$ & 49 & $7,0 \%$ & 0 & $0,0 \%$ & 0 & $0,0 \%$ \\
\hline Dr. & 139 & $19,8 \%$ & 127 & $18,1 \%$ & 10 & $1,4 \%$ & 2 & $0,3 \%$ \\
\hline Magister & 1 & $0,1 \%$ & 1 & $0,1 \%$ & 0 & $0,0 \%$ & 0 & $0,0 \%$ \\
\hline Prof. & 23 & $3,3 \%$ & 23 & $3,3 \%$ & 0 & $0,0 \%$ & 0 & $0,0 \%$ \\
\hline Prof. Dr. & 4 & $0,6 \%$ & 4 & $0,6 \%$ & 0 & $0,0 \%$ & 0 & $0,0 \%$ \\
\hline Student & 486 & $69,1 \%$ & 482 & $68,6 \%$ & 0 & $0,0 \%$ & 4 & $0,6 \%$ \\
\hline Summe & $\mathbf{7 0 3}$ & $\mathbf{1 0 0 , 0} \%$ & $\mathbf{6 8 7}$ & $\mathbf{9 7 , 7 \%}$ & $\mathbf{1 0}$ & $\mathbf{1 , 4} \%$ & $\mathbf{6}$ & $\mathbf{0 , 9 \%}$ \\
\hline \hline
\end{tabular}

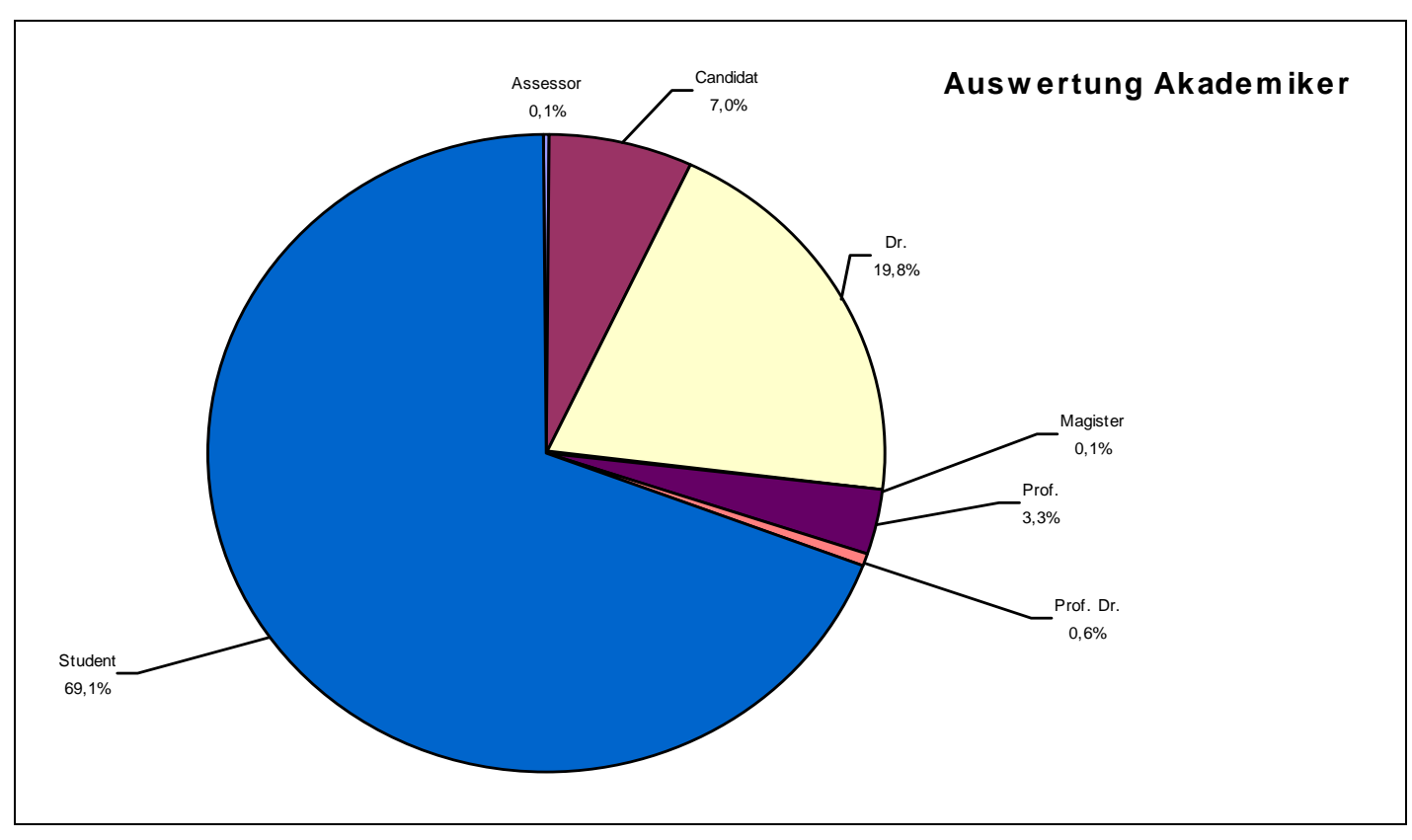


Verhältnis Besucher mit / ohne akademische(r) Ausbildung

\begin{tabular}{|c|c|c|c|}
\hline Monat & Akademiker & Alle & $\begin{array}{c}\text { Nicht } \\
\text { Akademiker }\end{array}$ \\
\hline 01 & 3 & 35 & 32 \\
\hline 02 & 21 & 71 & 50 \\
\hline 03 & 44 & 189 & 145 \\
\hline 04 & 62 & 225 & 163 \\
\hline 05 & 48 & 257 & 209 \\
\hline 06 & 62 & 369 & 307 \\
\hline 07 & 75 & 477 & 402 \\
\hline 08 & 139 & 553 & 414 \\
\hline 09 & 110 & 405 & 295 \\
\hline 10 & 91 & 263 & 172 \\
\hline 11 & 33 & 182 & 149 \\
\hline 12 & 15 & 73 & 58 \\
\hline
\end{tabular}

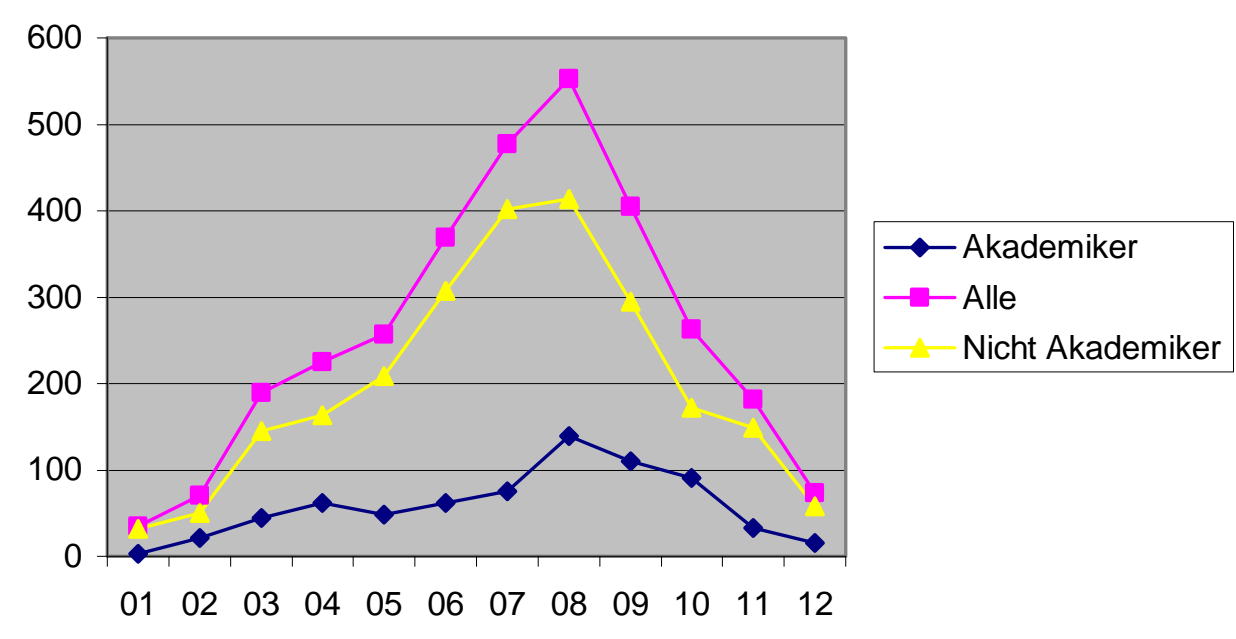




\section{Wiederkehrende Besucher}

Um wiederkehrende Besucher aus den Datensätzen zu filtern wurden folgende Bedingungen verwendet:

- Einträge stammen von verschiedenen Tagen

- Gleicher Nachname

- Anfangsbuchstabe des Vornamens ist gleich

- Ortsangabe ist gleich

In der daraus resultierenden Liste wurde dann per Hand überprüft, ob die Einträge nicht doch von verschiedenen Personen stammen. Damit ergeben sich:

\begin{tabular}{|r|r}
\hline Anzahl Besuche & \multicolumn{1}{|c}{ Anzahl Besucher } \\
\hline 4 & 1 \\
\hline 2 & 21 \\
\hline Summe & 22 \\
\hline \hline
\end{tabular}

\begin{tabular}{|c|c|c|c|c|c|}
\hline Nachname & Vorname & Besuche & Besuche & Beruf & aus \\
\hline Hülfemann & D. & 4 & $\begin{array}{l}04.10 .1810, \quad 30.09 .1811, \\
24.09 .1818,23.09 .1819\end{array}$ & Schuldirektor & Osterrode \\
\hline Bartels & & $2(?)$ & 03.05.1816, 25.09.1816 & & Göttingen \\
\hline Drever & H. & 2 & 05.06.1811, 27.09.1818 & & Uslar \\
\hline Druffel & Aug. von & 2 & 11.05.1817, 15.08.1818 & & Münster \\
\hline Erdmann & Erich & 2 & 19.04.1815, 04.09.1815 & Student Jur. & Nienburg \\
\hline Heinrichs & $\mathrm{C} \mathrm{H}$ & 2 & 12.02.1818, 24.07.1819 & $\begin{array}{l}\text { Student Theolo- } \\
\text { gie }\end{array}$ & $\begin{array}{l}\text { Burgdorf bei } \\
\text { Hannover }\end{array}$ \\
\hline Kaufholz & Georg: August: & $2(?)$ & 25.05.1818, 12.06.1818 & Student Jur. & \\
\hline Kern & & 2 & $04.10 .1810,30.08 .1811$ & $\begin{array}{l}\text { Hypotheken Con- } \\
\text { servateur }\end{array}$ & Osterrode \\
\hline Köhler & & 2 & 01.03.1814, 04.04.1814 & Kaufmann & Hannover \\
\hline Leopold & J. F. C. & 2 & 09.08.1814, 17.08.1814 & $\begin{array}{l}\text { Student Theolo- } \\
\text { gie }\end{array}$ & \\
\hline Mansfeld & & $2(?)$ & 22.10.1815, 11.08.1816 & Apotheker & Braunschweig \\
\hline Meissner & A. & $2(?)$ & 19.05.1816, 25.05.1818 & Kaufmann & Nürnberg \\
\hline Merrem & & $2(?)$ & 02.10.1815, 16.04.1817 & Professor & Marburg \\
\hline
\end{tabular}


Anhang B: Auswertung des Besucherbuchs

\begin{tabular}{|c|c|c|c|c|c|}
\hline Meyer & Fr. & $2(?)$ & 29.09.1815, 14.03.1818 & Kaufmann & Rinteln \\
\hline Mohring & Conrad & 2 & 08.08.1816, 20.10.1819 & Student Medizin & Nordhausen \\
\hline Müller & Ludewig & $2(?)$ & 06.04.1812, 21.08.1814 & Kaufmann & Salzderhelden \\
\hline Rettig & Friedr: Gottfr: & 2 & 18.06.1820, 12.03.1821 & $\begin{array}{l}\text { Student Theolo- } \\
\text { gie und Philoso- } \\
\text { phie }\end{array}$ & Hildesheim \\
\hline Schläger & & 2 & 13.07.1809, 17.08.1812 & Prediger/Pastor & Münden \\
\hline Schönstedt & Friedrich & $2(?)$ & 21.05.1819, 29.04.1820 & (Student Jur.) & Münster \\
\hline Schuster & & $2(?)$ & 15.03.1815, 20.09.1815 & Amtmann & $\begin{array}{l}\text { Scharzfeld am } \\
\text { Harz }\end{array}$ \\
\hline Tappezir & Wolf & 2 & 15.08.1818, 17.08.1818 & & Celle \\
\hline Wehmer & C: & $2(?)$ & 01.10.1809, 27.06.1813 & $\begin{array}{l}\text { Student Theolo- } \\
\text { gie }\end{array}$ & $\begin{array}{l}\text { Scharzfeld am } \\
\text { Harz }\end{array}$ \\
\hline
\end{tabular}

Die mit (?) versehenen Einträge könnten auch 2 verschiedene Personen sein. 


\section{Abkürzungen}

\begin{tabular}{|c|c|}
\hline Abb. & Abbildung \\
\hline $\mathrm{ADB}$ & Allgemeine Deutsche Biographie \\
\hline AVkG & Archiv des Instituts für Völkerkunde Göttingen \\
\hline AWG & Akademie der Wissenschaften zu Göttingen \\
\hline bes. & besonders \\
\hline bspw. & beispielsweise \\
\hline bzw. & beziehungsweise \\
\hline d.h. & das heißt \\
\hline ebd. & ebenda \\
\hline EN & Endnote \\
\hline FN & Fußnote \\
\hline Ggr. & Gute Groschen \\
\hline $\mathrm{H}$. & Heft \\
\hline Hg. & Herausgeber \\
\hline Mgr. & Mariengroschen \\
\hline NDB & Neue Deutsche Biographie \\
\hline N.F. & Neue Folge \\
\hline o.ä. & oder ähnliche(s) \\
\hline o.J. & ohne Jahresangabe \\
\hline o. Autor & ohne Autorenangabe \\
\hline o.O. & ohne Ortsangabe \\
\hline r. & recto \\
\hline Rt[h]lr. & Reichst[h]aler \\
\hline s. & siehe \\
\hline sic & $\begin{array}{l}\text { Gibt an, dass ein voranstehendes Wort in dieser (falschen) Form gele- } \\
\text { sen worden ist. }\end{array}$ \\
\hline Sp. & Spalte \\
\hline SUB Gö. & Niedersächsische Staats- und Universitätsbibliothek Göttingen \\
\hline $\mathrm{T}[\mathrm{h}] \mathrm{lr}$. & T[h]aler \\
\hline u.a. & unter anderem / und andere \\
\hline u.a.m. & und andere mehr \\
\hline u.s.f. & und so fort \\
\hline UAG & Universitätsarchiv Göttingen \\
\hline v. & verso \\
\hline v.a. & vor allem \\
\hline verw. & verwitwet \\
\hline vgl. & vergleiche \\
\hline z.B. & zum Beispiel \\
\hline Z.T. & zum Teil \\
\hline
\end{tabular}




\section{Quellen- und Literaturverzeichnis}

\section{Ungedruckte Quellen}

AWG Scient 236.

AVkG, Akte 1 (Altakte), Lose Blattsammlung.

AVkG, Besucherbuch des Akademischen Museum zu Göttingen (1808-1837).

AVkG, Catalogus Musei Academici (1778).

UAG Kur 3198, Übersicht und Beschreibung der zur Universität gehörenden Gebäude (1867).

UAG Kur 4 V g 1, Der vorgeschlagene Ankauf eines Naturalien-Cabinetts, 1756.

UAG Kur 4 V g 3, Der Ankauf des Grätzelschen Naturalien-Cabinetts, 1773.

UAG Kur 4 V g 4, Das Verzeichnis der Naturalien, womit die verwittwete Fürstin von Waldeck das Museum beschenkt hat, 1778.

UAG Kur 4 V g 5, Ernennung der Aufseher des Museums, 1778.

UAG Kur 4 V g 6, Die von Sr. Kgl. Majestät dem dortigen Museum geschenkten, bei hiesiger Bibliothek bisher aufbewahrten Erzstufen und Drusen, auch großen Silberstufen, 1777.

UAG Kur 4 V g 7, Die Vervollständigung der Sammlung durch Erzstufen vom Harze, 1780 .

UAG Kur 4 V g 8, Die von Sr. Majestät dem Könige von Dänemark geschenkte egiptische Mumie, 1781.

UAG Kur 4 V g 9, Der Ankauf des Stelznerschen Naturalien-Cabinetts; Naturaliengeschenke von Baron von Asch, 1781-1782.

UAG Kur 4 V g 11, Systematische Verzeichnisse des Museums, 1778.

UAG Kur $4 \mathrm{~V}$ g 12, Der Ankauf der Naturalien-Sammlung des IngenieurHauptmanns Müller, 1790.

UAG Kur 4 V g 13, Der Ankauf der zoologischen Sammlung des Medicinal-Raths Brandis zu Hildesheim, 1791.

UAG Kur 4 V g 14, Die Übersendung eines im Amte Ottersberg im Moor gefundenen alten Kahns für das Museum, 1794.

UAG Kur 4 V g 16, Der Antrag der Witwe des Leibchirurgus Lampe wegen Ankaufs des von ihrem Manne gesammelten Cabinetts, 1803.

UAG Kur 4 V g 17, Die dem Prof. Heeren ertheilte Erlaubnis, bei seinem Cursus über Ethnographie und Geographie seine Zuhörer ein paar Mal in die Zimmer des Museums führen und den für jene Wissenschaften brauchbaren Apparat vorzeigen zu dürfen, 1803.

UAG Kur 4 V g 19, Die Naturalien-Cabinet-Akten aus der Zeit der westphälischen Occupation, 1808-1813.

UAG Kur 4 V g 20, Die Revision und Abnahme der Rechungen des NaturalienCabinetts, 1814.

UAG Kur 4 V g 21, Die Rücklieferung der während westphälischer Ursupation im Jahre 1809 aus dem Casseler Museum erhaltenen naturhistorischen Gegenstände, 1814.

UAG Kur 4 V g 22, Der Antrag des hiesigen Ordens-Genealogisten Schädtler zum Ankauf eines prachtvollen Natur- und Kunst-Cabinetts, 1817. 
UAG Kur 4 V g 23, Die von dem Pastor Hess zu Nienburg geschenkten zoologischen Seltenheiten, 1819.

UAG Kur 4 V g 24, Die Kosten der von Paris erhaltenen Gipsabgüsse, 1823.

UAG Kur 4 V g 25, Das Gesuch des Handschuhmachers Klener sen. zu Göttingen um Anstellung als Ausstopfer von Vögeln und Säugethieren, 1823.

UAG Kur 4 V g 26, Die bei Mulsum im Lande Wursten im Moor aufgefundenen Alterthümer von Gold, 1823.

UAG Kur 4 V g 27, Das Anerbieten wegen Ankaufs der von dem Hofrath Merrem zu Marburg hinterlassenen Sammlung von ausgestopften Thieren, 1825.

UAG Kur 4 V g 28, Der Ankauf der Naturalien-Sammlung des Hofraths Ebel, 18261827.

UAG Kur 4 V g 29, Der bei dem akademischen Museum angestellte Assistent, 1827.

UAG Kur 4 V g 31, Die Ernennung des Wärters Weißleder am Museum, 1828.

UAG Kur 4 V g 32, Die Aufbewahrung der im Amte Esens beim Torfgraben aufgefundenen Gold- und Silbermünzen, 1829.

UAG Kur 4 V g 33, Das zum Verkauf ausgebotene Naturalien- und Kunst-Cabinet des weil. Consist. Raths Canonicus de la Tour zu Hildesheim, 1829.

UAG Kur 4 V g 34, Der Ankauf einer Sammlung Metallgegenstände von dem Chaussee-Inspector Horn, 1832.

UAG Kur 4 V g 35, Der in Antrag gebrachte Ankauf der Mineralien-Sammlung des weil Hofraths Bouterweck, incl. die vom Amts-Assessor Römer in Hildesheim zum Ankaufe dargebotene Sammlung von Versteinerungen, 1834-1838.

UAG Kur 4 V g 36, Die zu Fickmühlen, Amt Bederkesa, gefundenen alten römischen Münzen, 1836.

UAG Kur 4 V g 37, Die dem Prof. Berthold gestattete Benutzung der zoologischen Abtheilung des Museums, incl. die demselben aufgetragene Teilnahme an der Direction desselben, 1836.

UAG Kur 4 V g 38, Die für die Säcular-Feier bei dem akademischen Museum getroffenen Anordnungen und Einrichtungen, 1837.

UAG Kur 4 V g 39, Das Regulativ wegen des Zutritts der Studierenden zu dem akademischen Museum, 1838.

UAG Kur 4 V g 40, Die im Ihlsmoore, Gericht Delm, aufgefundenen und für das Museum angekauften Alterthümer, 1838.

UAG Kur 4 V g 41, Die Aussetzung einer Summe behuf Unterhalts und Vervollständigung des akademischen Museums, incl. die Anordnungen hinsichtlich der Scheidung in vier Abtheilungen unter besonderen Directoren: 1. mineral. u. geol. 2. ethnogr. 3. zool. 4. anthropol.-zootom, 1838.

UAG Kur 4 V g 42, Ein bei Lamstedt im Bremischen aufgefundenes Waffenstück, 1839.

UAG Kur 4 V g 43, Das Gesuch des Stud. Schlothauber um Anstellung als Conservateur am academ. Museum, und die demselben bewilligten Beihülfen, 1839.

UAG Kur 4 V g 44, Das zu Gölenkamp im Bentheimschen aufgefundene alterthümliche Gefäß, 1840.

UAG Kur 4 V g 45, Die zum Kaufe angebotenen naturhistorischen Sammlungen des Hofmedicus Dr. Zincken, genannt Sommer zu Braunschweig, 1840.

UAG Kur 4 V g 46, Der Ankauf der naturhistorischen Sammlungen des weil. OberMedicinal-Raths Blumenbach, incl. die Transferirung der Bilder-Gallerie in das neue Universitäts-Gebäude, 1840-1841. 


\section{Gedruckte Quellen}

JeAn Le Rond D'Alembert, Einleitung zur Enzyklopädie. Durchgesehen und mit einer Einleitung herausgegeben von Günther Mensching, Hamburg 1997.

DENis Diderot/JEAN Le Rond D'AlemberT, Encyclopédie ou Dictionnaire Raisonné des Sciences, des Arts et des Métiers, par une Société de Gens de Lettres. [17 Text- und 11 Tafelbände], Paris 1751-1772.

ARnOld AdOlPh Berthold, Transplantation der Hoden, in: Archiv für Anatomie, Physiologie und wissenschaftliche Medizin (1849), 42-46.

JoHANN CHRISTOPH ADELUNG, Grammatisch-kritisches Wörterbuch der Hochdeutschen Mundart, mit beständiger Vergleichung der übrigen Mundarten, besonders aber der Oberdeutschen, Wien 1811.

JoHANN FRIEDRICH BluMENBACH, De Generis Humani Varietate Nativa. Liber. Cum Figuris Aeri Incisis. 1. Auflage Göttingen 1776, 2. Auflage Göttingen 1782, 3. Auflage Göttingen 1795.

JoHANN FRIEDRICH BlumenBACH, Handbuch der Naturgeschichte. Mit Kupfern. [1. Theil], Göttingen 1779.

Johann Friedrich BlumenBach, Handbuch der Naturgeschichte. Mit Kupfern. [2. Theil], Göttingen 1780.

JOHANN FRIEDRICH BLUMENBACH, Einige Nachrichten vom academischen Museum zu Göttingen, in: Annalen der Braunschweig-Lüneburgischen Churlande 1 (1787) H. 3, 84-99.

JoHANN FrIEDRICH BlumENBACH, Einige Nachrichten vom academischen Museum zu Göttingen, in: Annalen der Braunschweig-Lüneburgischen Churlande 2 (1788) H. 2, 25-35.

JoHANN FRIEDRICH BlumenBACH, Beyträge zur Naturgeschichte. Theil 1, Göttingen 1790.

JoHANN FRIEDRICH BlumenBACH, Beyträge zur Naturgeschichte. Theil 2, Göttingen 1811.

GeORGES CuVIER/FranÇOIS Joseph Michel NoËL, Rapport sur instruction publique dans les nouveaux départemens de la basse Allemagne. Fait en exécution du Decret impérial du 13 décembre 1810.

CASPAR FRIEDRICH EINCKEL, Museographia oder Anleitung zum rechten Begriff und nützlicher Anlegung der Museorum, oder Raritäten-Kammern, Leipzig - Breslau 1727.

JoHANn REINHOLD FORSTER/GEORG FORSTER, Johann Reinhold Forster's der Rechte, Medicin und Weltweisheit Doctor, Professor der Naturgeschichte zu Halle. Mitglieds der Rußisch-Kaiserl. Akademie zu St. Petersburg, der Societäten der Wissenschaften, der Antiq. \& zu London, zu Madrit \& \&. Bemerkungen über Gegenstände der physischen Erdbeschreibung, Naturgeschichte und sittlichen Philosophie. Auf seiner Reise um die Welt gesammlet. Uebersetzt und mit Anmerkungen vermehrt von dessen Sohn und Reisegefährten Georg Forster, Professor am Carolino zu Cassel. Mit Landcharten, Berlin 1783.

Johann WolfGang vON GoETHE, Reise nach Göttingen und Pyrmont, in: Goethes Werke. Herausgegeben im Auftrage der Großherzogin Sophie von Sachsen, III. Abtheilung, 3. Band: Goethes Tagebücher 1801-1808. Fotomechanischer Nachdruck der Ausgabe Weimar, Böhlau 1887-1919 (1889), München 1987, 15-44.

Göttingische Anzeigen von Gelehrten Sachen (GGA). 
Karl August von HardenBerg, Karl August von Hardenberg 1750-1822. Tagebücher und autobiographische Aufzeichnungen, hrsg. von THOMAS STAMMKuHLMANN, München 2000.

ARnOLD H. L. HeEREN, Christian Gottlob Heyne. Biographisch dargestellt, Göttingen 1813.

Christian GotTlob Heyne, Die Jubelfeyer der Georg Augustus Universität zu Göttingen an ihrem funfzigsten Stiftungsfeste, dem 17 Septemb. 1787. Mit Beylagen, Göttingen 1787.

FRIEDRICH KARL GOTTLOB HIRSCHING (Hg.), Nachrichten von sehenswürdigen Gemälde- und Kupferstichsammlungen, Münz- Gemmen- Kunst- und Naturalienkabineten, Sammlungen von Modellen, Maschinen, physikalischen und mathematischen Instrumenten, anatomischen Präparaten und botanischen Gärten in Deutschland, nach alphabetischer Ordnung der Oerter. Dritter Band, Erlangen 1789.

THOMAS Hodgskin, Travels in the north of Germany: describing the present state of the social and political institutions, the agriculture, manufactures, commerce, education, arts and manners in that country; particularly in the kingdom of Hannover. Vol. 2, Edinburgh 1820.

IMMANUEL KANT, Von den verschiedenen Racen der Menschen. Zur Ankündigung der Vorlesungen der physischen Geographie im Sommerhalbenjahre 1775, Königsberg 1775.

D. JOHANN GEORG KRÜNITZ’s ökonomistisch-technische Encyclopädie, oder allgemeines System der Staats-, Stadt-, Haus- und Landwirtschaft und der Kunstgeschichte, in alphabetischer Ordnung, Bd. 98, Berlin 1805, 449-524.

Georg Christoph Lichtenberg, Schriften und Briefe, hrsg. v. Wolfgang ProMIES, 4 Bde, Frankfurt a.M. seit 1994.

GeOrg Christoph LichtenBerg, Etwas vom Academischen Museum in Göttingen, in: Göttinger Taschen Calender (1779), 45-57.

GEORG Christoph LiChTENBERg/GEORG FORSTER (Hg.), Göttingisches Magazin der Wissenschaften und Litteratur, Göttingen 1780-1785.

JoHann Daniel MajoR, Unvorgreiffliches Bedencken von Kunst- und NaturalienKammern insgemein, Kiel 1674.

KARL FRIEDRICH HEINRICH MARX, Zum Andenken an Johann Friedrich Blumenbach. Eine Gedächtniss-Rede gehalten in der Sitzung der Königlichen Societät der Wissenschaften den 8. Februar 1840, Göttingen 1840.

Georg HeinRich Oesterley, Johann Stephan Pütters Versuch einer academischen Gelehrten-Geschichte von der Georg-Augustus-Universität zu Göttingen Theil 4: Von 1820 bis zur ersten Säcularfeier der Universität im Jahre 1837. Mit 7 Kupfern, Göttingen 1838.

LORENZ OKEN (Hg.), Isis. Encyclopädische Zeitschrift, vorzügl. für Naturgeschichte, vergleichende Anatomie u. Physiologie, Leipzig 1817-1848.

AdAm Olearius, Gottorfische Kunstkammer, worinnen Allerhand ungemeine Sachen, so theils die Natur, theils künstliche Hände hervorgebracht und bereitet, Schleswig 1666.

Johann StePHAn PÜTTER, Johann Stephan Pütters Versuch einer academischen Gelehrten-Geschichte von der Georg-Augustus-Universität zu Göttingen. Theil 1, Göttingen 1765. 
Johann StePhan PÜtTer, Johann Stephan Pütters Versuch einer academischen Gelehrten-Geschichte von der Georg-Augustus-Universität zu Göttingen. Theil 2: Von 1765-1788, Göttingen 1788.

SAMUEL VON QUICCHEBERG, Inscriptiones vel tituli theatri amplissimi, complectentis rerum universitatis singulas materias et imagines eximias, ut idem recte quodque dici possit. Promptuarium artificiosarum miracolosarumque rerum, ac omnis raris thesauri et pretiosa supellectilis, structurae atque picturae quae hic simulin theatro conquiri consaluntur, ut eorum frequenti inspectione tractatione, singularis alique rerum cognito et prudentia admiranda, citò, facilé ac tutò comparari possit, Monachii 1565.

FRIEDRICH SAALFELD, Johann Stephan Pütters Versuch einer academischen Gelehrten-Geschichte von der Georg-Augustus-Universität zu Göttingen Theil 3: Von 1788-1820, Hannover 1820.

John Tradescant, Museums and their development. Vol. 1: Museum Tradescantianum: Or, a collection of rarities, preserved at South-Lambert neer London, London 1999 (Reprinted from the London: Grismond, 1656 ed.).

Michael Bernhard Valentini, Museum Museorum, Oder Vollständige SchauBühne Aller Materialien und Specereÿen Nebst deren Natürlichen Beschreibung, Election, Nutzen und Gebrauch, Aus andern Material-Kunst- und NaturalienKammern, Oost- und West-Indischen Reißbeschreibungen, Curiosen Zeit- und Tag-Registern, Natur- und Artzney-Kündigern, wie auch selbst-eigenen Erfahrung. Zum Vorschub Der Studirenden Jugend, Materialisten, Apothecker und deren Visitatoren, Wie auch anderer Künstler, als Jubelirer, Mahler, Färber, u. s. w. also verfasset, und Mit etlich hundert sauberen Kupfferstücken Unter Augen geleget Von D. Michael Bernhard Valentini, Franckfurt am Maeyn 1704.

LudWIG WALLIS, Der Göttinger Student. Mit acht Ansichten. Neudruck der Ausgabe von 1813, Göttingen 1913.

Ole WoRM, Museum Wormianum seu historia rerum rariorum tam naturalium, quam Artificialium, tam Domesticarum, quam Exoticarum, quae Hafniae Danorum in aedibus Authoris servantur, Amstelodami 1655.

JOHANN HeINRICH ZEDLER, Großes vollständiges Universal-Lexikon aller Wissenschaften und Künste, ... Bde 1-64, Suppl.bde 1-4, Nachdruck der Ausgabe Halle - Leipzig 1732-50 und 1751-1754, Graz 1961-1964.

\section{Literatur}

Max ARnim, Corpus Academicum Gottingense (1737-1928), Göttingen 1930.

MitChell G. AsH, Wissenschaftspopularisierung und bürgerliche Kultur im 19. Jahrhundert, in: Geschichte und Gesellschaft 28 (2002) H. 2, 322-334.

Christoph BeCKer, Vom Raritäten-Kabinett zur Sammlung als Institution. Sammeln und Ordnen im Zeitalter der Aufklärung, Egelsbach u.a. 1996.

GeORg W. BeHRE, Die Modellkammer der Universität Göttingen und deren Nutzung, in: Sächsische Landesstelle für Museumswesen (Hg.), Technische Modelle als Museumsbestand. Berichte und Erfahrungen, Chemnitz 1999, 4-13.

Tony Bennett, The Birth of the Museum. History, Theory, Politics, London u.a. 1997. 
Hans ERICh BöDeKer/Peter Hanns Reill/JÜrgen Schlumbohm (Hg.), Wissenschaft als kulturelle Praxis. 1750-1900, Göttingen 1999.

Christof Boehringer, Die Göttinger Universitäts-Münzsammlung, in: Hoffmann/Maack-Rheinländer (Hg.), „Ganz für das Studium angelegt“, 73-81.

CHRISTOF BOEHRINGER, Über die Göttinger Sammlung von Gipsabgüssen antiker Skulpturen, in: Hoffmann/Maack-Rheinländer (Hg.), „Ganz für das Studium angelegt", 64-72.

Wolfgang BöKer, Christian Gottlob Heyne und das Haus Papendiek 16 - ein Professor wird Göttinger, in: Göttinger Jahrbuch 50 (2002), 93-111.

Fritz Bolle, Berthold, Arnold Adolph, in: Neue Deutsche Biographie (NDB) 2, Berlin 1971 (Unveränderter Nachdruck der 1955 erschienenen ersten Auflage), 166.

Horst BREDEKAmp, Antikensehnsucht und Maschinenglauben. Die Geschichte der Kunstkammer und die Zukunft der Kunstgeschichte, Berlin 1993.

HoRst BREDEKAMP/JOACHIM BRÜNING/CORNELIA WEBER, (Hg.), Theater der Natur und Kunst. Wunderkammern des Wissens. Essays. Begleitband zur Ausstellung „Theatrum naturae et artis - Theater der Natur und Kunst. Wunderkammern des Wissens“, Martin-Gropius-Bau Berlin, 10. Dezember 2000-4. März 2001, Berlin 2000.

ConRad Bursian, Christian Gottlob Heyne, in: Allgemeine Deutsche Biographie (ADB) 12 (1880), Neudruck d. 1. Auflage Leipzig 1875-1912, Berlin 1967-71, , 375-378.

VICTOR CARUS, Johann Ludwig Gravenhorst, in: ADB 9 (1877), 616.

LORRAINE DASTON/KATHERINE PARK, Wunder der Kunst, Wunder der Natur, in: Dies. (Hg.), Wunder und die Ordnung der Natur 1150-1750, Frankfurt a.M. 2002, 301-354.

LORRAINE DASTON, Die kognitiven Leidenschaften: Staunen und Neugier im Europa der frühen Neuzeit, in: Dies. (Hg.) Wunder, Beweise und Tatsachen. Zur Geschichte der Rationalität, Frankfurt a.M. 2001, 77-98

LORRAINE J. DASTON, The factual sensibility, in: Isis 79 (1988), 452-470.

F.W.P. DOUGHERTY, Commercium epistolicum J.F. Blumenbachii: Aus einem Briefwechsel des klassischen Zeitalters der Naturgeschichte. Katalog zur Ausstellung im Foyer der Niedersächsischen Staats- und Universitätsbibliothek Göttingen, 1. Juni-21. Juni 1984, Göttingen 1984.

KeRSTIN DudLey, Historische Abbildungen der Völkerkundlichen Sammlung der Universität Göttingen aus der Zeit des Academischen Museums (1773-1840). (2 Bde.) (Unveröffentlichte Magisterarbeit), Göttingen 2001.

ReIMer EcK, Vom Pädagogium zur Keimzelle von Universität und Bibliothek. Zur Bau- und Nutzungsgeschichte des Pauliner-Klosters im 18. Jahrhundert, in: Elmar Mittler (Hg.), 700 Jahre Pauliner Kirche. Vom Kloster zur Bibliothek, Göttingen 1994, 145-149 und 160-163.

E[RNST HEINRICH] EHLERS, Göttinger Zoologen, Berlin 1901.

OsCAR FAMBACH (Hg.), Die Mitarbeiter der Göttingischen Gelehrten Anzeigen 17691836. Nach dem mit Beischriften des Jeremias David Reuß versehenen Exemplar der Universitätsbibliothek Tübingen, Tübingen 1976.

WALTHER FiSCHER, Johann Friedrich Ludwig Hausmann, in: (NDB) 8 (1969), $124 \mathrm{f}$.

Michel FoucAult, Die Ordnung der Dinge. Eine Archäologie der Humanwissenschaften, Frankfurt a.M. 2003 (zuerst: 1974). 
MARTIN GierL, Kompilation und die Produktion von Wissen im 18. Jahrhundert, in: Helmut Zedelmaier/Martin Mulsow (Hg.), Die Praktiken der Gelehrsamkeit in der Frühen Neuzeit, Tübingen 2001, 63-94.

Johann WolfGAng von GoETHE, Faust. Eine Tragödie. I. und II. Teil. Ungekürzte Ausgabe, München 1956.

Daniel Graepler, Die Originalsammlung des Archäologischen Instituts, in: Hoffmann/Maack-Rheinländer (Hg.), „Ganz für das Studium angelegt“, 55-63.

Andreas Grote (Hg.), Macrocosmos in Microcosmo. Die Welt in der Stube. Zur Geschichte des Sammelns 1450 bis 1800, Opladen 1994.

WiLHELM VON GÜMBEL, Johann Friedrich Ludwig Hausmann, in: ADB 11 (1880), 94-97.

Bente GundestruP, Det kongelige danske Kunstkammer 1737 = The Royal Danish Kunstkammer 1737, 3 Bde, København 1991-1995.

CARL HAASE, Göttingen und Hannover. Geistige und genealogische Beziehungen im ausgehenden 18. Jahrhundert, in: Göttinger Jahrbuch 15 (1967), 95-124.

Michael Hagner (Hg.), Ansichten der Wissenschaftsgeschichte, Frankfurt a.M. 2001.

Fritz Hartmann, Blumenbach, in: Rudolf Vierhaus/Hans Erich Bödeker (Hg.), Biographische Enzyklopädie der deutschsprachigen Aufklärung, München 2002, $35 f$.

Karl Julius Hartmann/Hans FÜCHSEL (Hg.), Geschichte der Göttinger Universitäts-Bibliothek. Mit 17 Lichtdrucktafeln, Göttingen 1937.

BrigitTA HAuser-SchäUblin/GundolF KRÜGer (Hg.), James Cook: Gifts and Treasures from the South Seas, Gaben und Schätze aus der Südsee. The Cook/Forster collection, Göttingen, München u.a. 1998.

Anke te Heesen/E. C. Spary (Hg.), Sammeln als Wissen. Das Sammeln und seine wissenschaftsgeschichtliche Bedeutung, Göttingen 2001.

Anke te Heesen/E. C. Spary, Sammeln als Wissen, in: Dies., Sammeln als Wissen, 7-21.

TheOdor InAma von SternegG, Johann Heinrich Gottlob von Justi , in: ADB 14, 747-753

Kunstmuseum des Landes Niedersachsen Herzog Anton Ulrich-Museum Braunschweig (Hg.), 250 Jahre Museum. Von den fürstlichen Sammlungen zum Museum der Aufklärung. Ausstellung in der Burg Dankwarderode, Braunschweig, 29. April bis 22. August 2004, München 2004.

CHRISTOPH HEYL, Einhorn und Indianermantel, in: Spektrum der Wissenschaft Spezial: Frühe Neuzeit. Forschung und Technik in der Renaissance 4 (2004), 12-15.

HANS-HEINRICH HimME, Stich-haltige Beiträge zur Geschichte der Georgia Augusta in Göttingen. 220 Stiche aus den ersten 150 Jahren der Göttinger Universität; zusammengetragen und mit Texten versehen anläßlich ihres 250-jährigen Jubiläums, Göttingen - Zürich 1987.

August Hirsch, Arnold Adolph Berthold, in: ADB 2 (1875), 512.

August HiRsCH, Karl Friedrich Heinrich Marx, in: ADB 20 (1884), 540-541.

DiETRICH HOFFMANN/KATHRIN MAACK-RHEINLÄNDER (Hg.), „Ganz für das Studium angelegt“. Die Museen, Sammlungen und Gärten der Universität Göttingen, Göttingen 2001. 
UlRich Hunger, Die Georg-August-Universität um 1800, in: Elmar Mittler (Hg.), „Der gute Kopf leuchtet überall hervor“: Goethe, Göttingen und die Wissenschaft, Göttingen 1999, 28-39.

Ulrich Hunger, Die Georgia Augusta als hannoversche Landesuniversität. Von ihrer Gründung bis zum Ende des Königreichs, in: Ernst Böhme/Rudolf Vierhaus (Hg.), Göttingen. Geschichte einer Universitätsstadt. Bd. 2: Vom Dreißigjährigen Krieg bis zum Anschluss an Preußen - Der Wiederaufstieg als Universitätsstadt (1646-1866), 139-213.

Oliver Impey/Arthur MacGregor (Hg.), The Origins of Museums. The Cabinet of Curiosities in sixteenth- and seventeenth-century Europe, Oxford 1986.

NiCOLAS JARDine, Sammlung, Wissenschaft, Kulturgeschichte, in: te Heesen/Spary, Sammeln als Wissen, 199-220.

ADRIENNE L. KAEPPLER, Die Göttinger Sammlung im internationalen Kontext, in: Hauser-Schäublin/Krüger (Hg.), James Cook, 86-93.

Friedrich Wilhelm KanTZEnBaCH, Georg Forsters Tochter. Therese, die blonde Polin, Saarbrücken-Scheidt 1989.

Thomas Klein, Königreich Hannover, in: Kurt G. A. Jeserich/Hans Pohl/GeorgChristoph von Unruh (Hg.), Deutsche Verwaltungsgeschichte. Bd 2: Vom Reichsdeputationshauptschluß bis zur Auflösung des Deutschen Bundes, Stuttgart 1983, 678-715.

ADOLF KLEINSCHMIDT, Blumenbach, Johann Friedrich, in: NDB 2 (1955), 329-330.

FRANZ-Ludwig KNEMEYER, Rheinbundstaaten 2. Die Verwaltungsorganisation des Königreichs Westphalen, in: Kurt G. A. Jeserich/Hans Pohl/Georg-Christoph von Unruh (Hg.), Deutsche Verwaltungsgeschichte. Bd 2: Vom Reichsdeputationshauptschluß bis zur Auflösung des Deutschen Bundes, Stuttgart 1983, 336339.

GUNDOLF KRÜGER, „Alles Geflechte besonders schön“: Begegnung mit Ethnographica des Academischen Museums, in: Mittler, Goethe, Göttingen und die Wissenschaft, 66-72.

GUNDOLF KRÜGER, Die Völkerkundliche Sammlung Göttingen. Eine Forschungsstätte an der Schnittstelle zwischen Museum und Universität. Vortrag, gehalten auf der Jahrestagung des Museumsverbandes für Niedersachsen und Bremen e.V. am 16. März 2000 in Göttingen, in: Mitteilungsblatt. Museumsverband Niedersachsen Bremen 59 (2000), 91-98.

NADINE KuBE, Ausstellung als Medium [unveröffentlichte Magisterarbeit], Göttingen 2004.

Thomas S. Kunn, Die Struktur wissenschaftlicher Revolutionen. Zweite, revidierte und um das Postscriptum von 1969 ergänzte Auflage, Frankfurt a.M. 1976.

HeINKE LANG, Ein Institut des Bildungsbürgertums? Die Anfänge des Göttinger Stadtmuseums [unveröffentlichte Staatsexamensarbeit], Göttingen 2004.

Georg LeyH, Chr. G. Heynes Eintritt in die Göttinger Bibliothek, in: Ders. (Hg.), Aufsätze. Fritz Milkau gewidmet, Leipzig 1921, 220.

KLAus-JOACHIM LORENZEN-SchmidT, Lexikon historischer Berufe in SchleswigHolstein und Hamburg, Kiel 1996.

Arthur MacGregor, Ark to Ashmolean. The story of the Tradescants, Ashmole and the Ashmolean Museum, Oxford 1983. 
DiETRICH VON MAERCKER, Die Zahlen der Studierenden an der Georg-AugustUniversität in Göttingen von 1734/37 bis 1978, in: Göttinger Jahrbuch 27 (1979), 141-158.

LUIGI MARINO, Praeceptores Germaniae. Göttingen 1770-1820, Göttingen 1995.

Oliver MATUSCHEK, „Hunde mitzubringen verbeten“. Besucher und Besucherbetreuung im Kunst- und Naturalienkabinett, in: Herzog Anton Ulrich-Museum Braunschweig, 250 Jahre Museum, 88-99.

GÜnTHer Mensching, Die Enzyklopädie und das Subjekt der Geschichte, in: Jean Le Rond d' Alembert, Einleitung zur Enzyklopädie. Durchgesehen und mit einer Einleitung herausgegeben von Günther Mensching, Hamburg 1997, XVI-XLIX.

Elmar MitTler (Hg.), 700 Jahre Pauliner Kirche: vom Kloster zur Bibliothek. Ausstellung in der Niedersächsischen Staats- und Universitätsbibliothek Göttingen, 10. Dezember 1994-15. Januar 1995, Göttingen 1994.

MoniKA MOMmerTZ, Schattenökonomie der Wissenschaft. Geschlechterordnung und Arbeitssysteme in der Astronomie der Berliner Akademie der Wissenschaften im 18. Jahrhundert, in: Theresa Wobbe (Hg.), Frauen in Akademie und Wissenschaft: Arbeitsorte und Forschungspraktiken 1700-2000, Berlin 2002, 31-63.

IRMGARD MÜSCH, „Nicht nur der Neugier allein, sondern auch der Natur Lehre nützlich“. Die Naturaliensammlung im Braunschweiger Kabinett, in: Herzog Anton Ulrich-Museum Braunschweig, 250 Jahre Museum, 61-69.

R.F. OvEnELL, The Ashmolean Museum, 1683-1894, Oxford 1986.

Julius LEOPOLD PAGEL, Wagner, Rudolf, in: ADB 40 (1896), 573f.

Susan M. PEARCE, Museums and their development. The European Tradition 17001900, London 1999.

WolfGang PetKe, Diplomatischer Apparat, in: Hoffmann/Maack-Rheinländer (Hg.), „Ganz für das Studium angelegt“, 82-90.

HANS PlisChKE, Das Institut für Völkerkunde an der Universität Göttingen. Aufbau und Geschichte, in: Göttinger völkerkundliche Studien 2 (1957), 7-9.

HANS PlischKe, Die Ethnographische Sammlung der Universität Göttingen. Ihre Geschichte und ihre Bedeutung, Göttingen 1931.

HANS PlisCHKE, Johann Friedrich Blumenbachs Einfluß auf die Entdeckungsreisenden seiner Zeit, Göttingen 1937.

Krzysztof Pomian, Collectionneurs, amateurs et curieux. Paris, Venise, XVIeXVIIIe siècle, Paris 1987.

Krzysztof Pomian (Hg.), Der Ursprung des Museums. Vom Sammeln, Berlin 1988.

KrZYSZTOF Pomian, Zwischen Sichtbarem und Unsichtbarem: Die Sammlung, in: Ders., Ursprung, 13-72.

Krzysztof Pomian, Für eine Geschichte der Semiophoren. Anmerkungen zu den Vasen der Medici-Sammlungen, in: Ders., Ursprung, 73-90.

Lewis Pyenson/Susan Sheets-Pyenson, Showing: Museums, in Dies. (Hg.): Servants of nature: A history of scientific institutions, enterprises and sensibilities, London 1999, 125-149. 
Helmut Rohlfing, Eine neue russische Bibliothek in Göttingen. Georg Thomas von Asch als Förderer der Georgia Augusta, in: 300 Jahre St. Petersburg: Russland und die „Göttingische Seele“; Ausstellung in der Paulinerkirche Göttingen unter der Schirmherrschaft von Bundespräsident Johannes Rau und dem Präsidenten der Russischen Föderation Wladimir Putin. - Göttingen: Niedersächsische Staats- und Universitäts-Bibliothek, Göttingen 2003, 287-302.

NicOlaAs RupKE (Hg.), Göttingen and the Development of the Natural Sciences, Göttingen 2002.

NicolaAs RupKe, The Göttingen Location, in: Ders., Göttingen, 19-32.

GÜNTER SCHEEL, Kurbraunschweig und die übrigen welfischen Lande, in: Kurt G. A. Jeserich/Hans Pohl/Georg-Christoph von Unruh (Hg.), Deutsche Verwaltungsgeschichte. Bd 1: Vom Spätmittelalter bis zum Ende des Reiches, Stuttgart 1983, 741-763.

EliSABETH Scheicher, Die Kunst- und Wunderkammern der Habsburger. Herausgegeben von Christian Brandstätter, Wien - München - Zürich 1979.

KuRT SCHELlEnBERG, Der Borheck-Bau und seine Vorläufer: Beitrag zur Grundrißgeschichte der Göttinger Universitäts-Bibliothek (1767-1787), in: Universitätsbibliothek Göttingen (Hg.), Beiträge zur Göttinger Bibliotheks- und Gelehrtengeschichte, Göttingen 1928, S. 55-74.

JuliUS VON SCHLOSSER, Die Kunst- und Wunderkammern der Spätrenaissance. Ein Beitrag zur Geschichte des Sammelwesens, Leipzig 1908.

WERNER SEIDEL, Baugeschichte der Niedersächsischen Staats- und Universitätsbibliothek in Göttingen 1734-1953, Göttingen 1953.

GöTZ VON SELLE, Die Georg-August-Universität zu Göttingen 1737-1937, Göttingen 1937.

James J. SheEhan, Geschichte der deutschen Kunstmuseen. Von der fürstlichen Kunstkammer zur modernen Sammlung, München 2002.

SusAn SHEETs-PyEnSON, Cathedrals of science. The development of colonial natural history museums during the late nineteenth century, Kingston u.a. 1988.

StefAn SIEMER, Geselligkeit und Methode. Naturgeschichtliches Sammeln im 18. Jahrhundert, im Erscheinen.

Bonnie G. SMith, The Practices of Scientific History, in: Dies. (Hg.), The Gender of History: Men, Women, and Historical Practice, Cambridge/Mass. u.a. 1998, 103-129.

RudOLF STICHWEH, Zur Entstehung des modernen Systems wissenschaftlicher Disziplinen. Physik in Deutschland 1740-1890, Frankfurt a.M. 1984.

HILDEGARD STUMPF, Christian Wilhelm Büttner, in: NDB 3 (1957), 6f.

MANFRED URBAN, 200 Jahre Göttinger Cook-Sammlung, Göttingen 1982.

MANFRED URBAN, 1. Frühe Auswertung des „Ethnographischen Apparates“ im Hochschulunterricht zu Beginn des 19. Jahrhunderts, in: 250 Jahre GeorgAugust-Universität Göttingen. Ausstellung im Auditorium 19. Mai-12. Juli 1987, Göttingen 1987, 158-160.

MANFRED URBAN, Die Erwerbungsgeschichte der Göttinger Sammlung, in: HauserSchäublin/Krüger (Hg.), James Cook, 56-85.

MANFRED URBAN, Die Völkerkundliche Sammlung, in: Hoffmann/MaackRheinländer (Hg.), „Ganz für das Studium angelegt“, 91-98.

Claudia VAlter, Studien zu bürgerlichen Kunst- und Naturaliensammlungen des 17. und 18. Jahrhunderts in Deutschland, Aachen 1995. 
Claudia Valter, Kunst- und Naturalienkabinette in der ersten Hälfte des 18. Jahrhunderts, in: Herzog Anton Ulrich-Museum Braunschweig (Hg.), 250 Jahre Museum, 21-30.

VANDENHOECK \& RupRECHT/DEuERLiCHSCHE BuCHHANDLung (Hg.), Mehr als irgend eine andere in Deutschland bekannt. Die Göttinger Universität im Bericht des „Universitätsbereisers“ Friedrich Gedicke aus dem Jahre 1789. Mit einer Vorbemerkung von Hartmut Boockmann [Sonderdruck], Göttingen 1996.

HAN F. VeRMeulen, Gatterer und Schlözer: Völkerkunde in der Aufklärung in Göttingen in den Jahren 1770, Leiden 1990.

RudOlF VIERHAUS/HANS ERICH BÖDEKER, Biographische Enzyklopädie der deutschsprachigen Aufklärung, München 2002.

Silke Wagener, Pedelle, Mägde und Lakaien. Das Dienstpersonal an der GeorgAugust-Universität Göttingen 1737-1866, Göttingen 1996.

Dietmar WiLloweIt, Die Universitäten, in: Kurt G. A. Jeserich/Hans Pohl/GeorgChristoph von Unruh (Hg.), Deutsche Verwaltungsgeschichte. Bd 1: Vom Spätmittelalter bis zum Ende des Reiches, Stuttgart 1983, 369-383.

Charles Withers, The Geography of Scientific Knowledge, in: Rupke, Development of the Natural Sciences, 9-18.

Denise WitTwer Hesse, Die Familie von Fellenberg und die Schulen von Hofwyl. Erziehungsideale, „Häusliches Glück“ und Unternehmertum einer Bernischen Patrizierfamilie in der ersten Hälfte des 19. Jahrhunderts, Bern 2002.

HeIDE Wunder, „Er ist die Sonn’, sie ist der Mond“: Frauen in der Frühen Neuzeit, München 1992.

CARLA YANNI, Nature's Museums: Victorian science and the architecture of display, Baltimore 1999.

Helmut Zedelmaier/Martin Mulsow (Hg.), Die Praktiken der Gelehrsamkeit in der frühen Neuzeit, Tübingen 2001.

\section{Internet-Ressourcen}

http://gdz-srv3.sub.uni-goettingen.de/cache/toc/D110377.html (Zugriff am: 1. Januar 2005)

http://www.h-net.org/ museum/ (Zugriff am: 1. Januar 2005).

http://www.mpiwg-berlin.mpg.de/research.html (Zugriff am: 18. Dezember 2004).

http://www.stadtarchiv.goettingen.de/chronik/1770.htm (Zugriff am 1. Januar 2005). http://www.stadtarchiv.goettingen.de/chronik/1781.htm (Zugriff am 1. Januar 2005). http://www.user.gwdg.de/ uhwg/Projekte.html (Zugriff am: 18. Dezember 2004). http://www.vl-museen.de/m-online/ (Zugriff am: 1. Januar 2005).

http://www.whonamedit.com/doctor.cfm/1247.html (Zugriff am: 16. November 2004). 


\section{Abbildungsverzeichnis}

Abbildung 1: Naturalienkabinett des neapolitanischen Apothekers Ferrante Imperato (um 1599); Frontispiz zu FERRANTE IMPERATO, Historia Naturale, Neapel 1599 (hier als Titelkupfer zur 2. Auflage, Venedig 1672).

Abbildung 2: Grundriss der zweiten Etage des späteren Universitätsgebäudes (1733); entnommen aus: REIMER ECK, Vom Pädagogium zur Keimzelle von Universität und Bibliothek. Zur Bau- und Nutzungsgeschichte des Pauliner-Klosters im 18. Jahrhundert, in: Elmar Mittler (Hg.), 700 Jahre Pauliner Kirche. Vom Kloster zur Bibliothek, Göttingen 1994, 145-149 und 160-163, 160.

Abbildung 3: Erdgeschoss des Kollegienhauses (ca. 1734); entnommen aus: KURT SCHELlENBERG, Der Borheck-Bau und seine Vorläufer: Beitrag zur Grundrißgeschichte der Göttinger Universitäts-Bibliothek (17671787), in: Universitätsbibliothek Göttingen (Hg.), Beiträge zur Göttinger Bibliotheks- und Gelehrtengeschichte, Göttingen 1928, S. 55-74, Taf. 5.

Abbildung 4: Blick auf das Universitätsensemble (von Norden): links das Kollegienhaus, hinten die Paulinerkirche, rechts die Professorenhäuser (1747); entnommen aus: PÜTTER, Gelehrten-Geschichte (1), 9.

Abbildung 5: Blick auf die ehemaligen Professorenhäuser (von Norden), die von 1793 an das Academische Museum und weitere Universitätssammlungen beherbergten (1816); Aquarellierte Federzeichnung von Friedrich Besemann, 21,8 x 34,6 cm; Städtisches Museum Göttingen, Inventar-Nr. 1933/23. Ich danke Herrn Barsky vom Städtischen Museum Göttingen für die Abdruckgenehmigung.

Abbildung 6: Besucherbuch des Academischen Museums, Einträge vom 16. Juli 1813 bis zum 2. November 1813; AVkG, Photographie: privat. Ich danke dem Kustos der Ethnologischen Sammlung, Herrn Dr. Krüger, für die Abdruckgenehmigung. 